\title{
Sociolinguistic Variation in Hong Kong Sign Language
}

by

Wai Yan Rebecca Siu

A thesis

submitted to the Victoria University of Wellington

in fulfilment of the

requirements for the degree of

Doctor of Philosophy

in Linguistics

Victoria University of Wellington

2016 



\section{Abstract}

Internal lexical variation appears to be a prominent feature within signed languages; it is perhaps a result of their distinctive acquisition patterns and fragile transmission. Recent research in different signed languages indicates that sociolinguistic variation within signed languages parallels some patterns found in spoken languages, though with some factors distinct to the former. This research examines sociolinguistic variation in a regional sign language, Hong Kong Sign Language (HKSL), "spoken” by deaf people in Hong Kong. The focus of this dissertation is lexical variation and two phonological variations in the signs DEAF/HEARING, and 'location drop' in articulation of signs made at the forehead.

This research project is a modified replication of the earlier studies in American Sign Language, Australian Sign Language, and New Zealand Sign Language (Lucas, Bayley, \& Valli, 2001; Schembri, McKee, McKee, Pivac, Johnston, \& Goswell, 2009; McKee \& McKee, 2011). The data of 65 participants recruited from the researcher's networks in the HKSL community using the friend-of-a-friend method was analyzed. Three types of data were collected: free conversation, picture naming and interview. A set of 120 pictures (with/without Chinese characters) was used to elicit signs for the concepts represented. Fifty-one out of these 120 concepts were analyzed from the semantic domains of colour, kinship, number, 
and country/region. Results show that school attended and age of signer play a prominent role in lexical variation. A gender effect is also found in several concepts. In addition to individual lexical items, the use of compound signs, 'citation forms' and handedness in number signs were also examined. Various social factors including school, age, gender, education, and work environment, interact with each other to constrain the variant choices. While numbers over ten can be produced either one-handed or two-handed, signs for hundred and tens highly favour the latter.

Regarding phonological variation, conversation videos of 40 participants were annotated for the DEAF/HEARING and location drop variables. For the DEAF/HEARING variables, preliminary investigation of the movement pattern demonstrates that there may be two different types of change going on: linguistically driven (originated from compounds) and socially driven (motivated by redefining deaf identity). It also suggests that DEAF is in a further stage of development than HEARING. For the location variable, twenty tokens from each participant were coded, producing 800 tokens for multivariate analysis. Again, complex correlations between social factors are found to constrain the lowering of signs. The findings further indicate that this change has originated in the deaf school name signs due to their salience, and signers from these schools have led the change. In addition, the results in both phonological variables show that grammatical constraints play an essential role in conditioning variant choices, which parallels results of the previous studies.

In sum, the lexical analysis results affirm the crucial role of school in shaping a signer's lexicon. The findings of the phonological variables confirm the role of grammatical function. Also, there is evidence of language change in progress in this young sign language. 
In memory of YELLOW YOUNGER，黃樂儀 



\section{Acknowledgements}

在此衷心感謝每一位參與本研究的香港壟人, 以及協助我的兩位好朋友 : 林國福和余安琳。

First of all, I would like to sincerely thank all the 68 participants and my deaf friends, Angel Lam and Anita Yu, who assisted me during my fieldwork. Without the help from you all, this research project could not have been carried out.

My special appreciation goes to my supervisors: Dr. David McKee, Dr. Rachel McKee (2013-2014), and Prof. Miriam Meyerhoff (2014-2016). I would like to express my heartfelt gratitude to Mr and Mrs McKee who have not only guided and inspired me in my work over the past years, but have also taken care of me in my personal life. My thanks goes to their boys as well. They have always made me feel very welcome at their home. The McKee family is like my family in New Zealand (sorry Rachel, I know you are too young to be my mother). I am tremendously grateful to Miriam for her guidance and patience throughout this project. Her encouragement and commendation has always motivated me to feel confident and self-assured. Thanks to all three of my supervisors for walking through this journey with me.

I gratefully acknowledge the financial support from VUW, both for my study and for my field trip for data collection. 
A big thank you to some other people who have helped me in my work in various ways:

- to Becky Yau, for spending a night in Cha discussing lexical variants in HKSL with me, while she should be enjoying her visit to Wellington in 2013.

- to Dr. Adam Schembri, for sharing his experience with me and giving me valuable advice when we met at NWAV-AP3 in 2014.

- to Dr. Daniel Ezra Johnson, for his time and patience in answering my numerous enquiries about working with Rbrul.

- to Dr. Paul Allison, for responding to my question promptly, which cleared up my confusion in a timely way, so that I could move forward in my analysis.

- to Dr. Lisa Woods, for her time and statistical support.

- to Andre Geldenhuis, for his time in helping me with LaTeX.

- to Neda Salahshour, for checking my references.

I would like to thank the members of the DSRU: Mark, Micky, and Sara, for allowing me to use their office, for teaching me NZSL, and for their patience in signing with me. The administrative staff of LALS, especially Janet and Bernie, thank you for making my life as a student easier. My core officemates, Neda and Yen, thank you for always being there when I wanted to chat and being quiet when I needed to work. My Cantonese interlocutors, Matthew and Fiona, thank you for chatting with me in my mother tongue. This is really important to someone away from home. Also, I am so thankful to have two HK deaf friends, Becky and Polly, around in NZ. Their visits and messages meant a lot to me. Special thanks to my best friend, sk, for always being there to share my ups and downs, and for all the support and encouragement. 
I would also like to acknowledge the roots of this research. My sincerest thanks go to Prof. Gladys Tang of CUHK and Prof. Ceil Lucas of Gallaudet University. I suppose Gladys had covertly "induced" me to study linguistics long before I became her masters student. Our encounter and conversations were always linked to the deaf people in HK and their language. I deeply appreciate her insights over the past years. Being in Ceil's sociolinguistics class and language variation class was the seed of this research project. Without her inspiration, this dissertation could not have been written.

To get to the stage of pursuing a $\mathrm{PhD}$ in the field of sign linguistics was never my initial expectation, but the result has been personally rewarding. Although I do not go to church anymore now, I will never forget how I first started learning to sign and meet deaf people. I am indebted to the members of the HK Catholic Pastoral Association for the Deaf, particularly the former pastor Fr. Charles Dittmeier, who opened my eyes to the Deaf World. I would like to extend my thanks to the HK deaf community. Every deaf individual I met during my interpreting career and my work at the CSLDS has inspired me in some way.

All my other friends, deaf and hearing, definitely deserve acknowledgement. I do not want to miss anyone of them in this list. Therefore, no names here, but their support and encouragement (plus countless free coffees and meals) over the past years are deeply appreciated and kept in my heart.

Finally, thanks to my family for their understanding. My parents have always allowed me to do whatever I want. Because of my big sister and little brother, I could do whatever I want without worrying my parents. We never talk about love in our family, but you all know I love you all. 



\section{How to access video materials for}

\section{this dissertation}

The reader has the option to read this dissertation in digital format with embedded videos if $\mathrm{s} /$ he chooses. In the disc attached at the back cover of this dissertation, there is a folder named videos and a pdf file of this dissertation. The pdf file is linked to the videos in the folder. In the pdf file, the figures that have captions in blue are linked to the corresponding videos. For example, on page 89 , Figure 4.3 as below. Click on the blue caption of Figure 4.3, then the video file of the two phonological variants of BLUE will be opened in a media player.

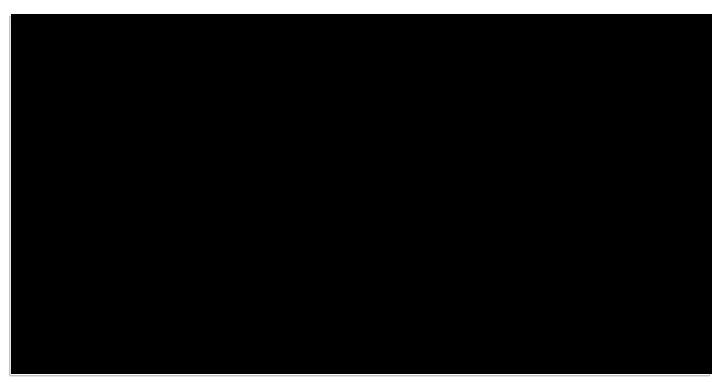

Figure 4.3: Phonological variants of BLUE 


\section{Contents}

$\begin{array}{ll}\text { Abstract } & \text { i }\end{array}$

Acknowledgements $\quad$ v

How to access video materials for this dissertation ix

Table of contents $\quad x$

List of Figures $\quad$ xiii

List of Tables $\quad$ xx

1 Introduction 1

1.1 Background ...................... 1

1.2 Aim and scope .................. 5

1.3 Thesis structure $\ldots \ldots \ldots \ldots$

2 Literature review $\quad 7$

2.1 Overview ..................... 7

2.2 Sociolinguistic variation in spoken languages . . . . . . 8

2.2.1 Lexical variation in spoken languages . . . . . . 8

2.2.2 Phonological variation in spoken languages . . . . . . 12

2.3 Sociolinguistic variation in signed languages . . . . . . . . 15

2.3 .1 Introduction . . . . . . . . . . . . . 15

2.3.2 Lexical variation in signed languages . . . . . . . 17 
2.3.3 Phonological variation in signed languages . . . . . 26

2.3 .4 The ASL study . . . . . . . . . . . . . . 30

2.3.5 The Auslan and NZSL studies . . . . . . . . . . . 36

2.4 Variation and documentation ... . . . . . . . . 43

2.5 Chapter summary . . . . . . . . . . . . . . . 46

3 Sociohistorical context for HKSL variation 47

3.1 Overview ....................... 47

3.2 History of deaf education in Hong Kong . . . . . . . . . . 48

3.3 Deaf schools in Hong Kong from mid-1930s to 2000s . . . . . 51

3.3.1 Old manual schools (OMS) . . . . . . . . . . 52

3.3.2 Hong Kong School for the Deaf (HSD) . . . . . . . . . 55

3.3.3 Victoria Park School for the Deaf (VSD) . . . . . . 58

3.3.4 Caritas Magdelene School for the Deaf (CMS) . . . . . 60

3.3.5 Lutheran School for the Deaf (LSD) . . . . . . . . . . 62

3.4 Language use in the deaf community . . . . . . . . . . 66

3.5 Language contact in the deaf community . . . . . . . . 69

3.6 Chapter summary . . . . . . . . . . . . . . 72

4 Research methodology $\quad 75$

4.1 Overview ............................. 75

4.2 Research approach . . . . . . . . . . . . . . 75

4.3 Participants and recruitment . . . . . . . . . 76

4.4 Data collection . . . . . . . . . . . . . . . 82

4.5 Data transcription and coding . . . . . . . . . . 87

4.5 .1 Lexical data . . . . . . . . . . . . . . 88

4.5 .2 Conversation data . . . . . . . . . . . . . 96

4.5 .3 Interview data . . . . . . . . . . . . . . . 112

4.6 Data analysis . . . . . . . . . . . . . . . . . 114 
4.7 Multicollinearity and collinearity . . . . . . . . . . 116

5 Lexical variation $\quad 121$

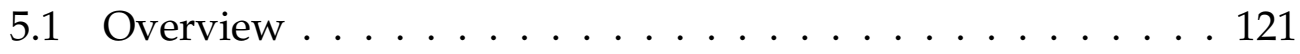

5.2 Extent of lexical variation . . . . . . . . . . . . . . . 122

5.3 Separate lexical variants and subvariants . . . . . . . . . 126

5.4 Variation according to social factors . . . . . . . . . . . 130

5.4 .1 School ....................... 131

5.4 .2 Age ......................... 169

5.4 .3 Gender . . . . . . . . . . . . . . . . . 184

5.4 .4 Other social factors . . . . . . . . . . . . 193

5.5 Use of possible homonyms . . . . . . . . . . . . . . . . . . . 194

5.6 Compound signs . . . . . . . . . . . . . . . . 196

5.7 'Citation form' . . . . . . . . . . . . . . . . . . 202

5.8 Numbers: one-handed versus two-handed . . . . . . . . 206

5.9 Language change in progress? . . . . . . . . . . . . . . . 211

5.10 Chapter summary . . . . . . . . . . . . . . . 216

6 Phonological variation 1: DEAF and HEARING 219

6.1 Overview . . . . . . . . . . . . . . . . . . . . . 219

6.2 Results of lexical data . . . . . . . . . . . . . . . . 221

6.3 Overall results of conversation data . . . . . . . . . . . . 227

6.3 .1 Linguistic factors . . . . . . . . . . . . . 233

6.3 .2 Social factors . . . . . . . . . . . . . . 236

6.4 Discussion . . . . . . . . . . . . . . . 240

6.4.1 Comparison with the ASL results . . . . . . . . . 240

6.4.2 Language change in progress? . . . . . . . . . . 242

6.4 .3 Potential implications . . . . . . . . . . . 246

6.5 Chapter summary . . . . . . . . . . . . . . . . . 248 
7 Phonological variation 2: location drop

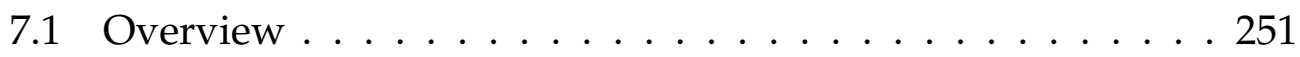

7.2 Results . . . . . . . . . . . . . . . . 252

7.2.1 Linguistic factors . . . . . . . . . . . 253

7.2 .2 Social factors . . . . . . . . . . . . . 257

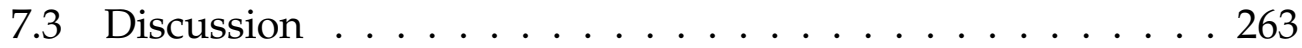

7.3.1 Comparison with ASL, Auslan, and NZSL results . . 264

7.3.2 Language change in progress? . . . . . . . . . . 272

7.3.3 The deaf school name signs . . . . . . . . . . 277

7.3.4 Deaf related work environment . . . . . . . . . . 280

7.4 Chapter summary . . . . . . . . . . . . . . 282

8 Conclusions and limitations 285

$\begin{array}{ll}\text { References } & 291\end{array}$

$\begin{array}{ll}\text { Appendices } & 313\end{array}$

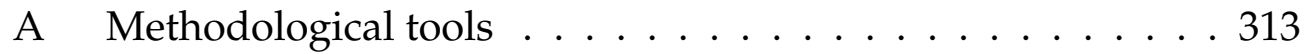

A1. Confidentiality agreement for Research Assistants . . . . 314

A2. Participant information sheet . . . . . . . . . 316

A3. Participant consent form . . . . . . . . . . . . 318

A4. Participant demographic profile . . . . . . . . . . . 320

A5. Interview questions . . . . . . . . . . . . 322

B Number of tokens per concept . . . . . . . . . . . . . 324

C List of compound signs . . . . . . . . . . . . . . . 325

D Sign pictures from HKSL publications . . . . . . . . . . 326 


\section{List of Figures}

3.1 Overview of deaf education in Hong Kong (See Section 3.3

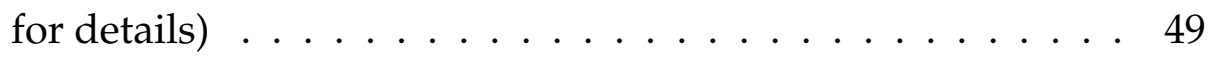

3.2 Hong Kong map showing the location of oral schools and clubs for deaf children . . . . . . . . . . . . . . 52

3.3 Contact signing continuum $\ldots \ldots \ldots 68 \ldots \ldots$

3.4 EMAIL . . . . . . . . . . . . . . . . . 69

4.1 Hong Kong map showing the five regions . . . . . . . . . . 81

4.2 Example of stimuli shown to participants . . . . . . . . 86

4.3 Phonological variants of BLUE . . . . . . . . . . . . 90

4.4 Separate lexical variants of BROWN . . . . . . . . . . 90

4.5 Phonological variants of GREEN . . . . . . . . . . . . 91

4.6 Compound variant of PINK . . . . . . . . . . . . . 93

4.7 Simplex variant of PINK . . . . . . . . . . . . 93

4.8 MOTHER in different HKSL publications . . . . . . . . . . . 94

4.9 ITALY in different HKSL publications . . . . . . . . . . . 95

4.10 Variants of THIRTEEN . . . . . . . . . . . . . . . 96

4.11 Examples of target signs: KNOW, GOVERNMENT and CRAZY 97

4.12 DEAF in ASL and HKSL . . . . . . . . . . . . . . 98

4.13 DEAF and MUTE in the first sign language book (1972) . . . . 99

4.14 DEAF in HKSL books published in different years . . . . . . 99

4.15 HEARING in the first sign language book (1972) . . . . . . . 100 
4.16 HEARING in HKSL books published in different years . . . . 100

4.17 GAY . . . . . . . . . . . . . . 106

4.18 THINK-neg . . . . . . . . . . . . . . . . . 107

4.19 Handshapes coded as unmarked . . . . . . . . . . . . . 111

5.1 Lexical variants of YELLOW . . . . . . . . . . . . . 125

5.2 Number of unique variants given by a single participant by school (CMS = 12 participants producing unique variants; $\mathrm{HSD}=12 ; \mathrm{LSD}=9 ; \mathrm{OMS}=10 ; \mathrm{VSD}=12) \ldots 133$

5.3 Examples of unique variants produced by single participant 134

5.4 Percentage distribution by school: GREEN (Fisher's Exact test,

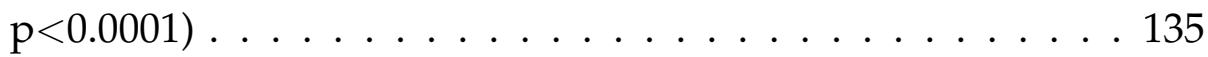

5.5 Percentage distribution by school: PINK (Fisher's Exact test,

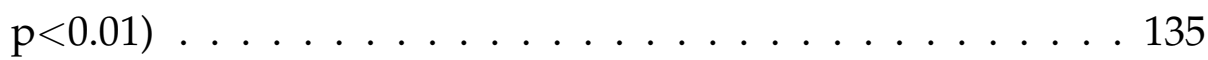

5.6 Major variants of PINK . . . . . . . . . . . . . . 136

5.7 Percentage distribution by school: GOLD (Fisher's Exact test,

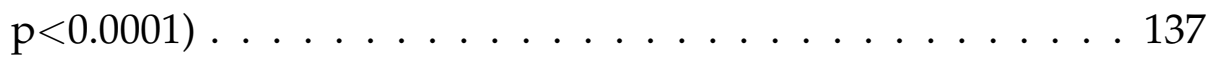

5.8 Percentage distribution by school: SILVER (CMS vs non-CMS, Fisher's Exact test, $\mathrm{p}<0.05) \ldots \ldots$. . . . . . . . . . 137

5.9 Phonological subvariants of GOLD . . . . . . . . . . 137

5.10 Variants of GOLD and SILVER with the same form . . . . . . 138

5.11 Percentage distribution by school: RED (HSD vs non-HSD,

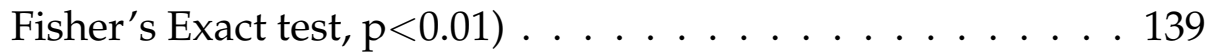

5.12 Percentage distribution by school: HUNG-HOM (HSD vs nonHSD, Fisher's Exact test, $\mathrm{p}<0.01) \ldots 139$

5.13 The most frequent variant: ORANGE1a . . . . . . . . . . 139

5.14 Phonological subvariants of ORANGE2 . . . . . . . . . . 140 
5.15 Percentage distribution by school: ORANGE (HSD vs nonHSD, Fisher's Exact test, $\mathrm{p}=0.05) \quad \ldots \ldots$. . . . . . . . 140

5.16 Variant used exclusively by OMS: HUNG-HOM2 . . . . . . . . 141

5.17 Major variants of PURPLE . . . . . . . . . . . . . . . 142

5.18 Percentage distribution by school: BLUE (LSD vs non-LSD, Fisher's Exact test, $\mathrm{p}<0.0001) \ldots \ldots$. . . . . . . . . . 142

5.19 Percentage distribution by school: PURPLE (CMS vs non-CMS, Fisher's Exact test, $\mathrm{p}<0.0001) \ldots \ldots$. . . . . . . . . . 142

5.20 Percentage distribution by school: GREY (Fisher's Exact test, $\mathrm{p}<0.0001) \ldots \ldots \ldots \ldots \ldots$. . . . . . . . . . . . . . . . . . . . . . .

5.21 Major variants of GREY . . . . . . . . . . . . . . . . 144

5.22 Phonological subvariants of FATHER and MOTHER . . . . . 145

5.23 Frequency distribution by school: FATHER (Fisher's Exact test, $\mathrm{p}=0.1478) \ldots \ldots \ldots \ldots$. . . . . . . . . . . . . . . . .

5.24 Frequency distribution by school: MOTHER (Fisher's Exact test, $\mathrm{p}<0.01) \ldots \ldots \ldots \ldots \ldots \ldots$. . . . . . . . . . . . . . . . .

5.25 Frequency distribution by school: ELDER-BROTHER (Fisher's Exact test, $\mathrm{p}=0.0903) \ldots \ldots \ldots$. . . . . . . . . . . . . . . . . . .

5.26 Frequency distribution by school: ELDER-SISTER (Fisher's Exact test, $\mathrm{p}=0.0677) \ldots \ldots \ldots \ldots \ldots$. . . . . . . . . . . . . . . .

5.27 Frequency distribution by school: YOUNGER-BROTHER (Fisher's Exact test, $\mathrm{p}=0.0524) \ldots \ldots$. . . . . . . . . . . . 149

5.28 Frequency distribution by school: YOUNGER-SISTER (Fisher's Exact test, $\mathrm{p}<0.01) \ldots \ldots$. . . . . . . . . . . . 149

5.29 Percentage distribution by school: AUSTRALIA (Fisher's Exact test, $\mathrm{p}<0.05) \ldots \ldots \ldots \ldots \ldots \ldots \ldots$ 
5.30 Percentage distribution by school: BEIJING (Fisher's Exact test, $\mathrm{p}<0.05) \ldots \ldots \ldots \ldots \ldots \ldots \ldots$

5.31 Percentage distribution by school: INDIA (Fisher's Exact test,

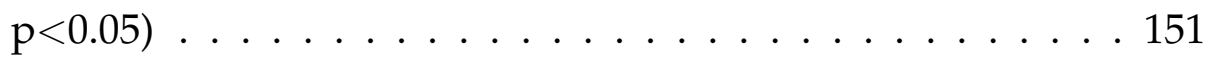

5.32 Three major variants of BEIJING . . . . . . . . . . . . 152

5.33 Three major variants of INDIA . . . . . . . . . . . . 153

5.34 Percentage distribution by school: KOREA (CMS vs non-CMS, Fisher's Exact test, $\mathrm{p}<0.001) \ldots \ldots$. . . . . . . . . 154

5.35 Percentage distribution by school: VIETNAM (HSD vs nonHSD, Fisher's Exact test, $\mathrm{p}<0.01) \ldots \ldots . \ldots . \ldots 154$

5.36 Variants of KOREA . . . . . . . . . . . . . . . 155

5.37 Variants of VIETNAM . . . . . . . . . . . . 155

5.38 Variants of THREE . . . . . . . . . . . . . . . 157

5.39 Variants of EIGHT . . . . . . . . . . . . . . . . 157

5.40 Frequency distribution by school: THREE . . . . . . . . . . 158

5.41 Frequency distribution by school: EIGHT . . . . . . . . . . 159

5.42 Variants of THIRTY . . . . . . . . . . . . . . . . . 161

5.43 Percentage distribution by school: TWENTY and THIRTY . . . 162

5.44 Major variants of FIFTEEN $\ldots \ldots \ldots$

5.45 Frequency distribution by school: FIFTEEN (Fisher's Exact test, $\mathrm{p}<0.001) \ldots \ldots \ldots \ldots$. . . . . . . . . . . . . . . . . . . .

5.46 Variants of TWENTY-ONE . . . . . . . . . . . . 165

5.47 SEVENa . . . . . . . . . . . . . . . . . 165

5.48 Percentage distribution by school: TWENTY-ONE (Fisher's Exact test, $\mathrm{p}<0.05) \ldots \ldots \ldots \ldots \ldots$. . . . . . . . . . . . . . .

5.49 Percentage distribution by school: TWENTY-TWO (Fisher's Exact test, $\mathrm{p}<0.05) \ldots \ldots \ldots \ldots \ldots$ 
5.50 Percentage distribution by school: TWENTY-FIVE (OMS vs non-OMS, Fisher's Exact test, $\mathrm{p}<0.05) \ldots 167$

5.51 Percentage distribution by school: TWENTY-SEVEN (Fisher's Exact test, $\mathrm{p}<0.05) \ldots \ldots \ldots$. . . . . . . . . . 167

5.52 New forms of ELEVEN . . . . . . . . . . . . . 175

5.53 Bound morphemes in sibling terms . . . . . . . . . . . 178

5.54 Minor variants of TAIWAN . . . . . . . . . . . . . 179

5.55 ITALY4 . . . . . . . . . . . . . . . . . . . 184

5.56 Phonological variants of NEW . . . . . . . . . . . 187

5.57 WIN/CHAMPION . . . . . . . . . . . . . . 190

5.58 Percentage distribution of all PINK variants by gender . . . 192

5.59 Percentage distribution of all PURPLE variants by gender . . . 192

5.60 Use of homonyms across different pairs . . . . . . . . . . . 194

5.61 Use of homonyms by social factors: school, age and gender . . 195

5.62 Frequency of compound signs by age groups . . . . . . . . . 197

5.63 Conditional inference tree for compound signs (age as continuous $\ldots \ldots \ldots \ldots \ldots$. . . . . . . . . . . . . . . . .

5.64 Conditional inference tree for compound signs (age as group) 201

5.65 Conditional inference tree for 'citation form' (age as contin-

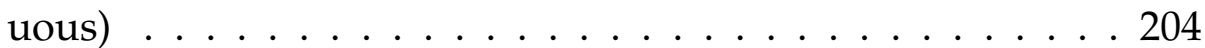

5.66 Conditional inference tree for 'citation form' (age as group) . 205

5.67 Conditional inference tree for handedness in number signs (age as continuous) . . . . . . . . . . . . 210

5.68 Conditional inference tree for handedness in number signs (age as group) . . . . . . . . . . . . . . 210

6.1 Variant HMH of DEAF and HEARING . . . . . . . . . . . 220

6.2 Variant MMM of DEAF and HEARING . . . . . . . . . 220 
6.3 Variant MH of DEAF and HEARING . . . . . . . . . . . 220

6.4 Variant M of DEAF and HEARING . . . . . . . . . . . . 221

6.5 Variant H of DEAF and HEARING . . . . . . . . . . . . 221

6.6 Frequency distribution of variants of DEAF (lexical data) . . . 223

6.7 Frequency distribution of variants of HEARING (lexical data) 223

6.8 Distribution of variants of DEAF by school (lexical data) . . . 224

6.9 Distribution of variants of HEARING by school (lexical data) . 224

6.10 Distribution of variants of DEAF by age (lexical data) . . . . . 225

6.11 Distribution of variants of HEARING by age (lexical data) . . 225

6.12 Distribution of variants of DEAF by gender (lexical data) . . . 226

6.13 Distribution of variants of HEARING by gender (lexical data) . 226

6.14 Frequency distribution of DEAF by individual participants

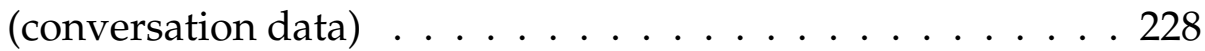

6.15 Frequency distribution of HEARING by individual participants (conversation data) . . . . . . . . . . . . 228

6.16 Percentage distribution of variants of DEAF (conversation data) . . . . . . . . . . . . . . . . . . . . 229

6.17 Percentage distribution of variants of HEARING (conversation data $) \ldots \ldots . \ldots . \ldots . \ldots . \ldots . \ldots$

6.18 Distribution of variants of DEAF by individual participants (conversation data $) \ldots \ldots . \ldots . \ldots . \ldots 231$

6.19 Distribution of variants of HEARING by individual participants (conversation data) . . . . . . . . . . . . . 232

6.20 Distribution of variants of DEAF by school (conversation data) (Fisher's Exact test, $\mathrm{p}<0.00001) \ldots . . \ldots 237$

6.21 Distribution of variants of HEARING by school (conversation

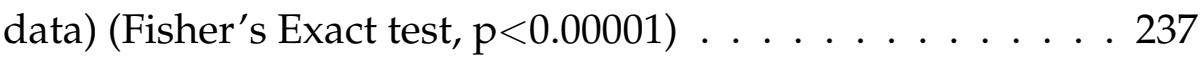


6.22 Distribution of variants of DEAF by age (conversation data)

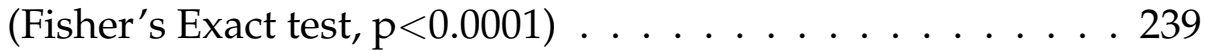

6.23 Distribution of variants of HEARING by age (conversation

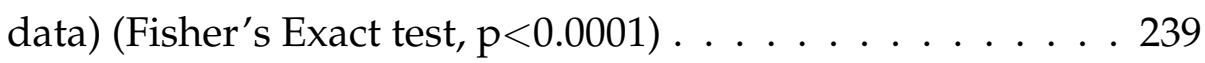

6.24 Distribution of variants of HEARING by gender (conversation data) (Fisher's Exact test, $\mathrm{p}<0.05) \ldots 239$

7.1 Citation form and non-citation form of THINK . . . . . . . . 252

7.2 Conditional inference tree for location drop (age as continuous) $(\mathrm{cf}=$ citation form; $-\mathrm{cf}=$ non-citation form $) \ldots . . . . .261$

7.3 Conditional inference tree for location drop (age as group) $(\mathrm{cf}=$ citation $\mathrm{form} ;-\mathrm{cf}=$ non-citation form $) \ldots . . . . .261$

7.4 Conditional inference tree for location drop (excluded deaf school name signs and age as continuous) ( $\mathrm{cf}=$ citation form; -cf=non-citation form) . . . . . . . . . . . . 262

7.5 Conditional inference tree for location drop (excluded deaf school name signs and age as group) ( $\mathrm{cf}=$ citation form; $\mathrm{cf}=$ non-citation form $) \ldots \ldots \ldots \ldots 26 \ldots \ldots \ldots$

7.6 Percentage of non-citation forms by age and grammatical class 274

7.7 Percentage of non-citation forms by age and school . . . . . 275

7.8 Percentage of non-citation forms by age and work environment 276

7.9 Percentage of non-citation forms by age and gender (excluding signers working in deaf-related environment) . . . . . 277

7.10 Percentage of non-citation forms by target sign . . . . . . 279 


\section{List of Tables}

2.1 Phonological variability in signed and spoken languages . . 27

3.1 Private deaf schools and clubs for deaf children before 1980 . 53

4.1 Demographic characteristics of 65 participants used for lexical variables . . . . . . . . . . . . . . . . 78

4.2 Demographic characteristics of 40 participants used for phono-

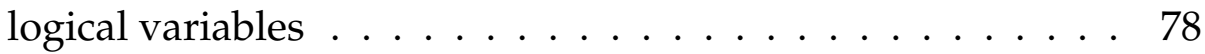

4.3 List of all target signs and their frequency . . . . . . . . . 110

4.4 VIF and tolerance of all social factor groups in the datasets for lexical analysis $(\mathrm{N}=65) \ldots \ldots . \ldots 118$

4.5 VIF and tolerance of all social factor groups in the datasets for phonological analysis $(\mathrm{N}=40) \ldots \ldots \ldots$

5.1 Number of extra tokens in each category . . . . . . . . . 122

5.2 Number of variants for each number sign . . . . . . . . . 123

5.3 Number of lexical variants per concept . . . . . . . . . . . . 124

5.4 Lexical variants and subvariants per concept . . . . . . . 128

5.5 Total number of variants by categories and schools . . . . . 131

5.6 Variants (both separate and subvariants) unique to age group 169

5.7 Number of variants by categories and age groups . . . . . 170

5.8 Examples of old variants and their frequency distribution by age ........................... 171 
5.9 Frequency distribution of major variants of RED by age . . . . 173

5.10 Frequency distribution of major variants of GREEN by age . . 173

5.11 Frequency distribution of major variants of PINK by age . . . 174

5.12 Frequency distribution of major variants of ORANGE by age . 174

5.13 Frequency distribution of twenties by age . . . . . . . . 176

5.14 Frequency distribution of kinship terms by age . . . . . . . . 177

5.15 Frequency distribution of major variants by age: TAIWAN . . 179

5.16 Frequency distribution of major variants by age: BEIJING . . 181

5.17 Frequency distribution of major variants by age: AUSTRALIA 182

5.18 Frequency distribution of major variants by age: INDIA . . 183

5.19 Frequency distribution of four sibling terms by gender . . . . 185

5.20 Frequency distribution of variants of BLUE1 by gender . . . . 186

5.21 Handshape use by gender in THREE, THIRTEEN, and TWENTYTHREE . . . . . . . . . . . . . . . . . 188

5.22 Handshape use by gender in EIGHT, EIGHTEEN, and TWENTY-

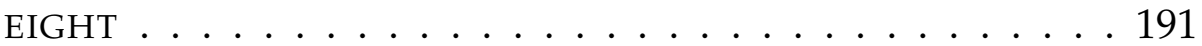

5.23 Results of Rbrul analysis of significant factor groups for compound signs . . . . . . . . . . . . . . . . . 198

5.24 Results of Rbrul analysis of significant factor groups for 'citation form' . . . . . . . . . . . . . . . 203

5.25 Results of Rbrul analysis of significant factor groups for twohanded number signs . . . . . . . . . . . . . 207

6.1 Distribution of variants of DEAF by gender for HSD signers

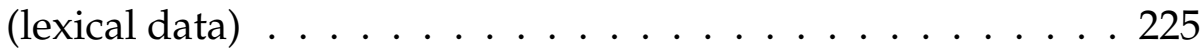

6.2 Numbers of participants producing the five variants (con-

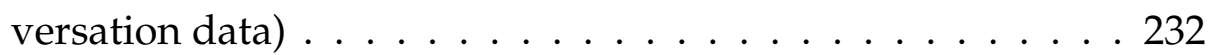


6.3 Contingency table for change in hand dominance and variants of DEAF (conversation data) $\ldots \ldots \ldots \ldots$

6.4 Contingency table for following segment and variants of HEARING (conversation data) . . . . . . . . . . . . . . . 234

6.5 Contingency table for following location and variants of HEARING (conversation data) . . . . . . . . . . . . . . 235

6.6 Contingency table for grammatical class and variants of DEAF (conversation data) . . . . . . . . . . . . . 236

6.7 Contingency table for grammatical class and variants of HEAR-

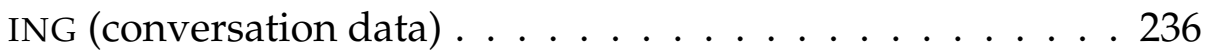

7.1 Results of Rbrul analysis of significant factor groups . . . . . 255

7.2 Results of Rbrul analysis of significant factor groups (excluded deaf school name signs) . . . . . . . . . . . 257

7.3 Number and percentage of non-citation form, school by target signs (deaf school name signs) . . . . . . . . . . 259

7.4 Comparison of seven possible linguistic factors on location drop in HKSL, ASL, Auslan, and NZSL (application value: non-citation form) . . . . . . . . . . . 266

7.5 Percentage of new forms at different stages of sound change 266

7.6 Social factors considered in HKSL, ASL, Auslan, and NZSL (application value: non-citation form, NB: studies differ in social factors examined $) \ldots \ldots$. . . . . . . . . 271 



\section{Chapter 1}

\section{Introduction}

\subsection{Background}

Sociolinguistics is the study of the relationships between language, culture and society, "with the goal being a better understanding of the structure of language and of how languages function in communication" (Wardhaugh, 2001). The breakthrough of sociolinguistics study over 50 years ago has led the field from traditional dialectology, which concerned mainly the correlation between dialect and geography, to variationist and interactional sociolinguistics (Chambers \& Trudgill, 1980; Chambers, 2009). Research conducted in the past decades has shown that language variation is not a free, random, unconstrained phenomenon as some early linguists had thought, rather it is structured, systematic and linked with social factors (Holmes, 2001; Chambers, 2009). Language indeed has its "orderly heterogeneity" (Weinreich, Labov, \& Herzog, 1968). Studying language variation is important not only because it contributes to the understanding of language change, but it also gives us an insight into complex social questions about 
language use (Meyerhoff, 2011).

Language variation is realized by means of linguistic variables. Linguistic variables can be found at different levels of linguistic structure, such as lexical, phonological, morphological, syntactic. Some of these variables co-vary with nonlinguistic independent variables of the social context, and hence are called "sociolinguistic variables" (Tagliamonte, 2012). Generally speaking, sociolinguistic variation in both spoken and signed languages is constrained by three types of factors. The first is linguistic or internal factors which include grammaticalization and phonological processes like assimilation. The second is social or inter-speaker factors, such as age, gender, region of origin, and socio-economic status. The last one is stylistic or intra-speaker, for example, the formality of the setting, and the characteristics of an interlocutor (Bell, 1984).

Recent research in different signed languages indicates that sociolinguistic variation within signed languages parallels some patterns found in spoken languages, though with some distinctive factors pertaining to the former. Quantitative studies of sociolinguistic variation in American Sign Language (ASL), Australian Sign Language (Auslan), New Zealand Sign Language (NZSL), British Sign Language (BSL), ${ }^{1}$ and Italian Sign Language (LIS) have shown that, apart from geographical and age factors, gender, ethnicity, and socio-economic class also correlate with lexical choices in varying degrees.

Although there is a growing number of variationist studies in signed

\footnotetext{
${ }^{1}$ The BSL study is part of the BSL corpus project: www.bslcorpusproject.org.
} 
languages, they are still scarce when compared with those that have examined spoken languages. Thus far, quantitative studies have mainly been conducted on national sign languages. This thesis explores variation in a regional sign language, Hong Kong Sign Language (HKSL). It is the language used by the deaf community in Hong Kong, a Special Administrative Region (SAR) of China. According to the official figures, there are around 3,400 deaf sign language users in Hong Kong (Census and Statistics Department, 2014). HKSL is not recognised as an official language (cf. NZSL) and in some social domains, access to interpreters is an issue. At the time of writing, the relative prestige of HKSL is complex (see Section $3.4)$.

It is evident that HKSL has a close relationship to the Nanjing/Shanghai variety of Chinese Sign Language (CSL) (Hong Kong Society for the Deaf, 1987; Woodward, 1993). It is the study of Woodward (1993) on the relationship between the sign language varieties in Hong Kong and Shanghai that triggered the research work on HKSL in the early 1990s. Later, work on the first HKSL dictionary began and it was published in 2007 (Tang, 2007). Since the outset of the dictionary project, systematic analysis of the linguistic properties of HKSL has been undertaken at the Chinese University of Hong Kong (CUHK). Before that, according to Tang (2007), some work of Yau (1977) and Van Hoek, Bellugi, and Fok (1986) had touched on HKSL, though no distinction between HKSL and CSL was made in their work.

In 2003, the Centre for Sign Linguistics and Deaf Studies (CSLDS) at CUHK was established, in response to the growing need for sign language research and training. Hitherto, most of the research has concentrated on 
formal description, and the language acquisition of deaf children. Only in recent years, studies on variation have begun (Centre for Sign Linguistics and Deaf Studies, 2015). In 2013, a small-scale project on lexical variation and diachronic change in HKSL was conducted and found that some signs have become "more symmetrical, fluid and centralized" and "simpler in structure" over time (Sze, Chu, Lo, \& Lo, 2013).

On the other hand, members of the HKSL community frequently complain that the language contains 'too much' variation. This is possibly because Hong Kong is a small and high-density city where language variation is expected to be limited. The existing diversity in HKSL is regarded as a problem by the government. The topic "Standardization of Sign Language" was raised in the government Rehabilitation Programme Plan (200507) and a working group was formed to address the issue. The focus of the group was then shifted to promoting the language rather than standardizing it. Nonetheless, variation in HKSL has yet to be empirically described, let alone studied from a sociolinguistic perspective.

I have been involved in the HKSL community for over 20 years. During this time, I worked as an interpreter (for 10 years) and taught HKSL (several years). I have been both frustrated and fascinated by the variation in HKSL. Attending sociolinguistics and language variation classes at Gallaudet University opened my eyes to how variation can be studied. By combining my experience and observation, together with variationist research methods, I hope to understand and report the variability in HKSL from a sociolinguistic perspective in this thesis. 


\subsection{Aim and scope}

This study investigates sociolinguistic variation in HKSL, focusing on its lexical and phonological levels. These two levels were chosen because lexical variation is the most obvious and anecdotally noted as an issue by the HKSL community. Variable phonological features were selected for study because they require relatively less native intuition to identify and code compared to morphological and syntactic features. Both linguistic (internal) and social (external) factors will be explored. The two phonological variables involved are (1) the signs DEAF and HEARING, which are known to have at least two variants each; and (2) location in the class of signs articulated at or near the forehead area, which are observed to be lowered in daily conversation. This is the first quantitative study with an attempt to empirically examine lexical and phonological variation in HKSL. As we will see in more detail in Chapter 3, the respondents studied here are a sample of deaf signers balanced roughly evenly by gender, age, and schooling background (i.e., the study includes signers from deaf schools based in different parts of Hong Kong and from different age cohorts). The thesis contributes to the understanding of variation in signed languages, a growing field in both sign linguistics and sociolinguistics in general.

The thesis aims to explore the possibility that the variability noticed by members of the HKSL community patterns in regular ways. Specifically, it asks:

1. What are the external social constraints on lexical and phonological variation in HKSL? Are they similar to or different from those identi- 
Introduction

fied and described in spoken languages and other signed languages?

2. How many phonologically related variants exist for DEAF and HEARING? How are these variants used? Are they conditioned by linguistic and/or social factors?

3. Is the location variation in the class of signs articulated at or near the forehead influenced by the phonological environment? Are they conditioned by other linguistic and/or social factors?

4. Is there language change in progress?

\subsection{Thesis structure}

The structure of this dissertation is organized in the following way. Chapter 2 gives a literature review on variation studies in spoken and signed languages, with particular focus on lexical and phonological variation related to the current project. The sociohistorical context for variation in HKSL is delineated in Chapter 3. This background information is important not only in understanding and interpreting the findings presented in later chapters, but also in conducting further cross-linguistic comparison. Chapter 4 presents the research methodology. I will first describe the research approach, characteristics of the participants and the recruitment process. Then I will detail how the data is collected, coded and analyzed. The issues of multicollinearity will also be explained. Chapter 5 reports and discusses the findings of lexical variation. The findings and discussions of the two phonological variables: DEAF/HEARING and location drop are given in Chapter 6 and Chapter 7 respectively. Finally, Chapter 8 concludes the findings of this study and discusses the limitations. 


\section{Chapter 2}

\section{Literature review}

\subsection{Overview}

When the field of sociolinguistics was developing in the 1960s, sign linguistics was also emerging. The pioneering work of William C. Stokoe asserted that the fundamental linguistic properties of natural signed languages are parallel to those of spoken languages, including having a sociolinguistic dimension. Like spoken languages, sociolinguistic variation in signed languages occurs at different linguistic levels including lexical, phonological, morphological, syntactic, and discourse (Bayley \& Lucas, 2011). Yet, there are some idiosyncratic issues linked with deaf signing communities that sociolinguists should be aware of in doing sign language research (Wilbur, 1987; Lucas \& Bayley, 2010).

In this chapter, I first review the literature about sociolinguistic variation in spoken and signed languages in Section 2.2 and Section 2.3 respec- 
tively. The sections focus on lexical and phonological levels. Since this research is a modified replication of three earlier studies in sign language variation, detailed accounts of the original studies are delineated. In Section 2.4, the issue of documentation is discussed for the reason that documentation of sign variants is a by-product of this study. The last section, Section 2.5 , gives a summary of the whole chapter.

\subsection{Sociolinguistic variation in spoken languages}

\subsubsection{Lexical variation in spoken languages}

Speakers of different English varieties use different words to mean the same thing. For instance, pavement is used in the United Kingdom (UK), sidewalk in the United States (US), and footpath in Australia (Svartvik \& Leech, 2006). In the dialects of American English, the list of regional lexical variants is counted in thousands, which covers a wide range of categories. Many of these words are the outcomes of various word-formation processes such as compounding, blending, and borrowing (Wolfram \& Schilling-Estes, 2006). In studying the campus English used by Anglophone university students in Cameroon, Kouega (2009) found that many non-standard lexical items were incorporated in the informal speech of these students. These variants sprang from a variety of word-formation processes, like borrowing, coinage, compounding, phraseological units, shortening, affixation, and meaning change. More recently, the Voices of the UK (VoUK) project of the British Library described the lexical variation in the BBC Voices Recordings, a set of group conversations about 
language, accent and dialect recorded in different locations across the UK. The project showed that different lexical items were used, and that these reflected not only the age and regions of the speakers, but also that some terms related to speaker interest or expertise, particular industries, cultural and leisure pursuits, and new influences from other languages (Robinson, 2012).

In fact, dialect atlases have been published to document the regional varieties in both the US and the UK, recording both phonological and lexical variants (Labov, Ash, \& Boberg, 2006; Upton \& Widdowson, 2006). However, work in linguistic variation and change has been focused more on phonology, syntax and discourse, whilst lexis has been relatively neglected (Beal, 2010; Chambers, Trudgill, \& Schilling-Estes, 2004). Nonetheless, many sociolinguistic researchers have demonstrated the importance and value of studying lexical variation (e.g., Armstrong, 2001; Chambers, 2000; Johnson, 1993; Nagy, 2011). As Lodge (2004) commented, "Far from being peripheral to sociolinguistic structure, 'words' approach the very core of the linguistic identity of the different social groups that make up any community" (p. 228).

Apart from regional variation, age related difference is another common finding in lexical variation studies. By applying the apparent-time construct, synchronic data can reflect diachronic linguistic development and language change in progress (Labov, 1994). That is to say, research showing different variant choice between old and young language users can be a plausible indicator of language change over time. Although issues like age-grading and stability of idiolect are substantial concerns, real-time comparisons generally support the apparent-time hypothesis, 
i.e., that synchronic differences in the distribution of variants across age groups is a reliable indication of change in progress (if not the actual rate of change) (Bailey, Wikle, Tillery, \& Sand, 1991; Bailey, 2004; Boberg, 2004; Pope, Meyerhoff, \& Ladd, 2007). Age-grading occurs when individuals change their linguistic behaviour at a particular age during their lifetimes, but there is no relevant change in the community as a whole. Such changes recur in successive generations and could appear in different age groups (Chambers, 2009; Eckert, 1997; Hockett, 1950). On the other hand, individuals can acquire new lexis and pronunciation throughout their lifetimes (Bayard, 1989; Chambers, 1992). This makes the study of lexical variation across generations problematic, given that the apparent-time method was designed for aspects of the linguistic system which are remain basically stable after language has been acquired (Labov, 1994).

In examining lexical variation and change in Canadian English, Boberg (2010) conducted an apparent-time analysis with the support of some realtime data. His results illustrated "the complex interplay of age-grading, late adoption and real-time change" (p. 195). Regarding the claim of Americanization of Canadian English words, examples of both convergence and divergence with American vocabulary were observed. In addition to this cross-border influence, some lexical variables exhibited changes related to cultural developments.

Adopting a real-time approach, Johnson (1993) analyzed the relationship between lexical variation and change by using comparable data from the mid-1930s and 1990. Besides regional and age-related variation, other social factors including sex, race, education, and rurality were also associated with lexical choices. However, $71 \%$ of the lexical items that exhibited 
changes were not related to any social factors. She suggested that such changes pertain to "cultural changes involving technology, the economy, education and information" (p. 301).

Differing patterns between males and females are often observed in studies of variation in linguistic structures other than lexis. Nevertheless, Bayard's study (1989) of American lexical diffusion in New Zealand is among the few that have shown sex-related differentiation. In examining the use of and preference for 27 pairs of lexical items, he found that women were slightly more conservative in usage, though no big difference was observed in general. More typically, Nagy (2011) reported a lack of systematic patterns of lexical variation across regions, age, and sex in her study of Faetar in Italy and Canada, a language contact situation. She proposed that lexical change is more evident in early stages of language contact and that a contact situation may cause resistance to change. She further suggested that lexical change may behave differently than structural changes.

Extending variationist study to non-native language users, Nadasdi, Mougeon, and Rehner (2008) investigated the variation patterns in the spoken French of second language learners. Their study focused on five variants of the concept car in spoken Canadian French used by immersion high school students with a variety of language backgrounds. Results showed that home language and lexical exposure were the main factors affecting variant choice.

To summarize, lexical variants originate from a variety of word-formation processes and lexical choices may reflect a speaker's interests, exposure to innovation, or even more. Research in spoken languages has shown that 
region, age, sex, race, education, rurality, language background, and lexical exposure are all possible social factors in predicting lexical variation. Yet, other possible effects on lexical change like cross-border influence, cultural developments, and situations of language contact should not be ignored. It is possible that lexical change proceeds in a different way than that of structural changes such as phonological change.

\subsubsection{Phonological variation in spoken languages}

At a finer level, speakers of different varieties pronounce the same word differently. For example, data is pronounced as /'dertə/ in the US, whereas in New Zealand, people say /'detə/. Indeed, variation occurs at every level of phonological representation (Kügler, Féry, \& Vijver, 2009). Labov (1966) investigated five phonological variables in the speech of New Yorkers. By employing quantitative methods and controlling for stylistic variation, he described how the variables correlated with social factors like social class, gender, ethnicity, and age. The study also presented evidence of change in the prestige norm (Labov, 1966). In addition, his findings showed a notable homogeneity in speech attitudes among speakers, despite the interindividual and intra-individual heterogeneity in their speech habits (Halliday, 1975).

However, the groundbreaking work of Labov has been criticized for assuming a linear phonetic dimension scale and a single non-standard to standard sociolinguistic continuum (Hudson, 1996; Knowles, 1978). In view of these limitations, James Milroy (1982) took different phonetic parameters into consideration when analyzing the complex vowel variables 
in data from the Belfast study. Instead of quantifying the variants based only on vowel height, he examined variation in rounding, backing, lengthening and diphthongization. He also used a range score to calculate the tendency of reduced allophones in exploring variation. While the workingclass speech had a wide range of phonological variation, the middle-class tended to converge on a single variant (Milroy, 1982; Milroy, 1987a). To address the problems with treating the social class as a continuum, Milroy (1987b) applied the concept of social network as an analytic tool. The results demonstrated a substantial and reliable relation between an individual's language use and his/her social network. Furthermore, the Milroys' studies suggested that a 'prestige' or 'standard' norm may not always be present (Milroy \& Milroy, 1978).

In Hong Kong, phonetic variation and change are observed in all components of Cantonese syllables, consonants, vowels, as well as tones (Bauer \& Benedict, 1997). Cantonese, like other Chinese languages, does not use affixes to the extent and in the way that English or other inflectional languages do. This lack of affixational morphology resembles most (if not all) signed languages (Lucas et al., 2001). Since the linear affixation in spoken language plays a role in phonological variation, it would be interesting to see what other linguistic factors (apart from immediate phonological environment) may constrain phonological variation in languages like Cantonese. Furthermore, it would be worth finding out if the most influential social factors of the speaking community are also relevant to the signing community in Hong Kong.

Among the list of phonological variables examined in Hong Kong Cantonese, four initial consonant alternations had raised much concern by re- 
searchers and these were $\mathrm{n}-/ 1-, \mathrm{y}-/ 0-, \mathrm{k}^{\mathrm{wh}}-/ \mathrm{k}_{-}$, and $\mathrm{k}-/ \mathrm{h}-$. Two pilot studies showed that young and male speakers were more innovative in their choices of the first three variables (Pan, 1981; Yeung, 1980). Despite the small sample size and lack of statistical testing, their findings suggested age and gender were significant social factors. Pan (1981) also demonstrated that these variables were in a prestige/non-prestige dichotomy, with $/ \mathrm{n} /, / \mathrm{y} /, / \mathrm{k}^{\mathrm{wh}} /$ as the prestige forms, which were produced more frequently when formality level increased in his data. A later quantitative study focused on three sociolinguistic variables: (n), (y), and (k), and was aimed to investigate non-linguistic factors including age, gender, ethnicity, education and speech register (Bourgerie, 1990). The last variable only occurs in the third-person pronoun / $\mathrm{keoi}^{5}$ / 佢 'he/she/him/her/it,' whereas the first two variables involve second and first-person pronouns: $/ \mathrm{nei}^{5}$ / 你 'you' and $/ \mathrm{yo}^{5}$ / 我 'me.' Again, gender and age appeared to be the most significant social factors, though different patterns were observed. Unlike the previous studies, females were found to be more innovative and used more non-prestige forms. In accordance with other studies, age was inversely correlated to the use of innovative forms. Older speakers were more conservative than younger speakers, but the critical boundaries of each variable were different. Speech register was also found to be significant, while as the level of formality increased, the use of nonprestige forms decreased. Although the original focus of the study was social factors, the data that emerged called for a look at word class ${ }^{1}$ as well. For the $(\mathrm{y}) /(0)$ variable, adverbs and verbs had a high propensity to be realized with the innovative form (0), while stative verbs had a much

\footnotetext{
${ }^{1}$ The five word classes coded were adverbs, verbs, stative verbs, pronoun and noun.
} 
lower tendency. As for the (n)/(l) variable, verbs, stative verbs, and adverbs showed a high tendency to be realized with (1), but the opposite in the case of demonstratives and particles.

\subsection{Sociolinguistic variation in signed languages}

\subsubsection{Introduction}

Unlike spoken languages, most signed languages are young, minority languages that are used in contact with a majority spoken language, although they are produced in a different modality. (Schembri \& Johnston, 2012). Discernible variation in signed languages is found to be influenced by the dominant spoken languages of the hearing community (Adam, 2012). Conditions for the acquisition of sign language for most deaf signers are different from the hearing population, who learn their native language from their parents. It is believed that at least $92 \%$ of deaf children have hearing parents who do not know sign language (Mitchell \& Karchmer, 2004). Most deaf signers learn sign language from their peers, or sometimes from their teachers, rather than their parents. Usually this is at a later age when they go to school, in particular, deaf schools (which are very often the place where most deaf people acquire sign language and build up their deaf identity). The transmission of sign languages is further weakened by educational policies for deaf children. In many countries, sign language has been forbidden as a medium of instruction in deaf education over the past century or so. Only during the last three decades, has the importance of sign languages in educating deaf children begun to be 
recognized and utilized (Plaza-Pust, 2012).

Internal lexical variation appears to be a prominent feature within signed languages; it is perhaps a result of their distinctive acquisition patterns and fragile transmission. It is believed that deaf schools have played an essential role in forming sign language communities, and to a certain extent, have contributed to age and regional variation (Johnston \& Schembri, 2007; Quinn, 2010; Stamp et al., 2014). Age variation is often the result of changing language policies and attitudes at deaf schools in different generations (Lucas, Bayley, \& Valli, 2001; McKee \& McKee, 2011). Regional variation is linked to the location of deaf schools and has been noticed in sign languages from various parts of the world, such as Flemish Sign Language (VGT) in Flanders, Belgium (Vanhecke \& Weerdt, 2004), Sign Language of the Netherlands (NGT) (Schermer, 2003), British Sign Language (BSL) (Sutton-Spence \& Woll, 1999; Quinn, 2010), American Sign Language (ASL) (Lucas et al., 2001), South African Sign Language (SASL) (Penn \& Reagan, 1994), Australian Sign Language (Auslan) (Johnston \& Schembri, 2007), New Zealand Sign Language (NZSL) (McKee \& McKee, 2011), and Taiwan Sign Language (TSL) (Chen \& Tai, 2009), among others.

On the grounds that this project is a modified replication of previous quantitative studies on ASL, Auslan, and NZSL, detailed description of their methods and findings will be given. Before recounting these studies, I first consider the literature on lexical variation and phonological variation in signed languages in the next two sections. 


\subsubsection{Lexical variation in signed languages}

While studies of lexical variation in spoken languages are quite often looking at lexical choice between a pair of standard and non-standard (vernacular) forms, or a pair of local and 'imported' forms (e.g., the diffusion of American vocabulary in other English varieties); the situation in signed languages is quite different. Instead of starting with dichotomous variants, research in signed languages usually starts from asking how many variants exist in the language.

As mentioned in the previous section, the majority of deaf individuals learn sign language in deaf schools. Hence, language policy shift from manual to oral in deaf education is crucial in shaping variation patterns in a sign language. For instance, due to the influence of ASL in education in Thailand in the 1950s, Woodward (1996) found that most urban signers below the age of 40 used Modern Standard Thai Sign Language (MSTSL). Though this had a certain amount of vocabulary in common with the two original sign languages used by older signers, the lexicon of MSTSL was closer to ASL than the Original Chiangmai Sign Language (OCSL) or Original Bangkok Sign Language (OBSL).

In countries like Ireland, where deaf boys and girls have been educated separately, gender variation is salient. Though gender difference is more striking in older generations, it continues to play a role in the lexicon of Irish Sign Language (ISL) and possibly beyond the lexical level (Leeson \& Grehan, 2004). When the educational language policy shifted from manual to oral in the mid-twentieth century, the transmission of ISL was interrupted and created generational variation (LeMaster, 2002). The opening 
of a new school in the mid-west region in 1979, the increasing number of deaf children educated in mainstream, and the influence of BSL in Northern Ireland have all been contributing factors to the regional variation in ISL (Leeson \& Saeed, 2012).

Gender variation has also been observed in Flemish Sign Language (VGT) used by signers in Flanders, the northern part of Belgium, where segregation of deaf boys and girls in schooling happened before the 1970s (Van Herreweghe \& Vermeerbergen, 2004). However, regional variation in Flanders is more conspicuous. The lexicographic study conducted by Vanhecke and Weerdt (2004) investigated the signs used in five regions where deaf schools were present. It was believed that five regional varieties of VGT had developed in and around the deaf schools. Despite the fact that lexical variation appeared within and among regions, the researchers concluded that an ongoing natural standardization process can be observed. Another study in VGT focused on the evolution of signs in all five regions. VGT signers from different age groups were recruited to investigate how the signs described in the sign language book printed in 1858 had evolved. The variation and change they noticed involved both parametric change and shift, meaning there was phonological change in form and total shift to a distinct form, with examples of the latter outnumbering the former. Another interesting finding in this study was that one of the schools, for which none of the deaf students in the study had deaf parents, was strongly influenced by Signed Dutch when compared with other schools. This was due to the lack of sign role models (deaf students with deaf parents) and hence, the deaf children mainly learnt Signed Dutch signs from their teachers and educators (Antoons \& Boonen, 2004). 
Intraregional variation is far from uncommon in signed languages, especially when a region has more than one deaf school. By comparing variant choice of 73 concepts from child and adult corpora data, Eichmann and Rosenstock (2014) investigated the role of deaf school in regional variation in German Sign Language (DGS). ${ }^{2}$ The study focused on the lexical variants used by pupils and adults who had graduated from three deaf schools in the same region, Saxony (southeast Germany). Statistical analysis showed a strong association between school and variant choice for 30 and 20 concepts in the adult and child data respectively. Results confirmed the link between deaf schools and variation in DGS and suggested this link was weaker in the younger generation.

A similar situation of intraregional variation exists if there is no deaf school in a region. When deaf children returned to their home area after studying in a residential school in other areas, they brought along their sign variants to the region. In examining regional variation in BSL in his home area, where no deaf school existed, Quinn (2010) found that lexical choice by signers reflected the location of schools they attended rather than where they lived. In particular, the colour signs one learnt in early school days were very likely be retained in one's adulthood.

A more recent large-scale study in BSL was based on corpus data. Stamp et al. (2014) investigated lexical variation and change in 41 lexical items of four semantic categories: countries, colours, numbers and UK

\footnotetext{
${ }^{2}$ Like many other parts of the world, most deaf children acquire DGS through peer interactions in deaf schools.
} 
place names. ${ }^{3}$ The corpus collected spontaneous and elicited data from 249 signers from eight cities across the UK. Taking signer's region as a reference, tokens of colour, country, and number signs were coded as 'traditional' or 'non-traditional,'4 whereas tokens of UK place names were coded as 'local' and 'non-local. ${ }^{5}$ Rbrul was used to perform multivariate analysis on these two datasets. Results showed that age, school location, and language background (i.e., signer with deaf parent(s) or not) were all significant predictors in the analysis of signs for countries, colours and numbers, with age as the strongest predictor. When these three semantic domains were analyzed separately, age was the only factor explaining variant choice of country signs; age and school location were predictors of colour signs; all three social factors: age, school location, and language background were predictors for number signs. The researchers suggested that signs for countries were subject to other external influence, like political correctness, attitudinal changes towards borrowing, and increased mobility internationally which resulted in greater transnational interaction. Hence, younger signers tended to use more 'non-traditional' variants. Regarding UK place names, results confirmed that local residents of the region were more likely to use local variants, showing different preferences among residents and non-residents (Stamp et al., 2014).

\footnotetext{
${ }^{3}$ Skinner (2007) is an earlier work on BSL number variations, but does not consider the interplay of social and linguistic factors which is the focus in the current study.

${ }^{4}$ Two methods were used to decided whether a token would be coded as 'traditional/non-traditional.' One was to categorise as 'traditional' forms used in teaching materials and earlier studies; another was if a local deaf fieldworker classified it as such. Uncertain tokens were excluded.

${ }^{5}$ Local deaf fieldworkers of different regions identified their 'local' variant. All variants other than the 'local' variant were coded as 'non-local.'
} 
Stamp, Schembri, Fenlon, and Rentelis (2015) further investigated the use of handedness in BSL number signs. A subset of number signs which had both one-handed and two-handed variants were coded for multivariate analysis. Three social factors: age, language background and gender were found to be significant. Old signers, signers with deaf parents, and male signers favoured two-handed forms. Young signers aged 16 to 39 disfavoured two-handed forms the most. Social class, school location and ethnicity were not significant factors in the use of two-handed number signs. They concluded their results were analogous with other research (e.g., McKee, McKee, \& Major, 2011) in two ways. Firstly, one-handed signs were more prevalent than two-handed signs. In their database, the former accounted for $86 \%$ of all tokens. Secondly, the reduction in use of two-handed signs among young signers indicated language change in progress, that is, a phonological shift from two- to one-handed signs was occurring over time (Stamp et al., 2015).

In another corpus-based study in Italian Sign Language (LIS), Geraci et al. (2011) selected ten lexical items for statistical analysis. ${ }^{6}$ This subset was chosen because of the phonological variation and/or diachronic change observed. The researchers aimed to investigate three processes of articulatory simplification, which caused phonological change in the lexicon, as suggested in earlier studies by Radutzky $(1989,2009)$. These three processes were: (1) neutralization of contact feature; (2) neutralization of neu-

\footnotetext{
${ }^{6}$ The study was part of a large-scale corpus project consisting of data from 165 signers recruited in 10 cities within 4 regions in Italy. Four types of data were collected to form the corpus: free conversation, individual narration, question-elicitation and picture-naming. The lexical variation study consisted of data elicited from the picture-naming task from 128 signers from 8 cities.
} 
tral space; (3) handshape assimilation. The first one referred to the change of a sign from having contact with the signer's face or body to one without contact. The second one concerned the place of articulation moving from the signer's body to neutral space. The last one included two operations: one involved the handshape of the non-dominant hand assimilating to the dominant hand in a two-handed sign; another one was the partial or full handshape assimilation of one stem to the other in a compound sign.

Based on these features, the relevant tokens (only tokens from seven out of the ten selected items were available) were coded as primitive form or derived form. Their multivariate analysis using VARBRUL revealed that age and gender were the most significant social factors, with non-old signers (combining young and middle-aged signers) and female signers using more derived forms. Motivated by the data collected, they also examined lexical variants. The more prevalent target variants (i.e., variants produced in all cities) were classified as national variants (which included the primitive and derived variants mentioned above), while other forms (i.e., variants that appeared in a few cities) were classified as local variants. The results of the analysis of nine out of the ten selected items (one item was excluded due to failure in elicitation) showed that older signers favoured the use of local variants and signers from the central region (e.g., Rome) preferred the national variants. Geraci et al. (2011) concluded that "a process of standardization seems to be taking place, with younger signers leading this way. An effect of gender and a leading role of the LIS variety signed in Rome are also attested" (p. 568).

Employing a real time approach, Frishberg (1975), Woll (1987), Chen and Tai (2009) compared sign language publications from different peri- 
ods to explore variation and historical change in ASL, BSL and TSL respectively. According to Frishberg (1975), formational changes in signs were motivated by two basic principles: ease of articulation and ease of perception. Both compound signs in ASL and BSL tended to assimilate two parts or retained only one part of a compound over time. While cases of both two-handed signs becoming one-handed and one-handed becoming two-handed were observed in ASL and BSL, only the second change was seen in TSL. Concerning parametric changes, similar patterns were reported in both ASL and BSL. For handshape, a two-handed sign became more symmetric by assimilating the non-dominant hand to the shape of the dominant hand. As for location, the centralization of signs or moving towards neutral space happened, except for signs articulated on the face, which were more likely to move from central to peripheral. On the other hand, the TSL data presented a different pattern. No tendency towards symmetry of handshape was found; while signs articulated at the body appeared to be centralized, signs made in the face area tended to be lowered. Regarding movement, all three languages exhibited an inclination to reduced, simplified, or smoothed movement in contributing to fluidity. Different and repeated movement changes were also observed in the TSL data. In the BSL data, handshape changes appeared more frequently than other parameters, whereas the TSL data suggested movement and location were more apt to change. It should be noted that two main resources were used for comparison in the work of ASL and TSL. The ASL materials were around 50 years apart and those of the TSL were between 7 and 20 years apart. On the other hand, 13 different sources of BSL over the past 150 years were collected for examination. Hence, the different patterns observed may be attributed to the different time periods over which change 
was observed.

In Hong Kong, Sze, Chu, et al. (2013) conducted a small-scale project on lexical variation and diachronic change in HKSL. They invited four old signers and six young signers to perform a lexical elicitation task. ${ }^{7}$ Their list for picture or video priming included 146 concepts, which had been observed to have variants. A total of 1,985 signs were collected and the authors identified 973 lexical variants. The number of variants for each concept ranged from two to thirteen. They further classified the signs into "older forms" and "more current forms." The "older" forms were those used by the old signers or those used in the conversation between the young signers and their parents; the "more current forms" were those used by the young signers with their peers. By examining the related phonological variants, Sze and her colleagues identified several phonological processes, such as assimilation and deletion, that contributed to the diachronic change in some lexical items. Over time, these signs had become "more symmetrical, fluid and centralized" and "simpler in structure." They claimed that these changes were rapid, "resulting in variants within and across generations of signers."

More recently, there has been research focusing on the lexicons used in two oral schools: Victoria Park School for the Deaf (VSD) and Caritas

\footnotetext{
${ }^{7}$ The old signers had graduated from the two earliest deaf schools and all the young signers were from the first deaf school and have deaf parents. The age of the old signers in their samples were mid-late 50s and the young signers were in their early-mid 30s.
} 
Magdelene School for the Deaf (CMS) ${ }^{8}(Y u, 2014)$. Adopting the modified Swadesh list, the study examined and compared 100 core lexical items used by 28 signers of various age cohorts. The majority of the participants had either gone to both schools (VSD for primary education and CMS for secondary education) or had just attended CMS (for both primary and secondary education). The informants were asked to recall the signs they used in VSD if they had studied in VSD. The results showed that the signs used in the two schools were highly related. The findings also suggested some lexical changes: change in location, reduction in movement, deletion in compounds and deletion of non-dominant hand. This was the first study that aimed to document signs used in schools other than the first deaf school and the old signing schools.

While it was encouraging to see research involving non-native signers of other schooling backgrounds, there were both theoretical and methodological issues with the study. Firstly, the research assumed there were two signing varieties, one of the VSD and one of the CMS. As an oral primary school where sign language was prohibited, by the time the students left VSD at the age of 11 or 12, their signing skills were still developing. As $\mathrm{Yu}$ (2014) reported, some target concepts were not found in VSD. It is very unlikely that there was a VSD signing variety. Secondly, the research method relied on the informants' ability to recall signs they used when they were young. This would certainly cause memory errors which means the findings must be interpreted with caution.

\footnotetext{
${ }^{8}$ The abbreviations VSD and CMS are used in consistency with those used in the next chapter. The abbreviations used in Yu's study were VPSD and CSD/CMS for the two schools.
} 


\subsubsection{Phonological variation in signed languages}

Over the past fifty years, different models of sign language phonology have been proposed, including the Cheremic Model (Stokoe, Casterline, \& Cronberg, 1965), the Hold-Movement Model (Liddell, 1984; Liddell \& Johnson, 1989), the Hand Tier Model (Sandler, 1986, 1989), the Moraic Model (Perlmutter, 1991), and the Prosodic Model (Brentari, 1998), among others. Despite the different view in representing the phonological structure of sign language, it is generally agreed that manual signs are composed of three main parameters: handshape (hand configuration), location, and movement. Battison (1978) suggested orientation as a fourth parameter because it is sometimes contrastive, while others argue that orientation is not an independent category like handshape, location, and movement, but a confined feature that can be conceived in relational terms with other features (Brentari, 1998; Sandler \& Lillo-Martin, 2006). Non-manual feature is another component of sign. Non-manual features include facial expressions, mouth actions, movements of head, eyebrows, cheek, or body. Some of these features carry meanings, and may perform specific grammatical functions. While not all signs require a non-manual signal, some signs are regarded as incorrect if a specific non-manual feature is missing. With regard to use of articulators, there are both one-handed signs and two-handed signs in the sign language lexicon. Interestingly, the two hands in a two-handed sign are not as independent as they seem to be. The non-dominant hand (weak hand) is always subordinate in some sense, and is constrained in its formational elements like handshape and movement (Brentari, 1998; Sandler \& Lillo-Martin, 2006). Sometimes, what is known as "weak drop" may occur, i.e., the deletion of the non-dominant 
hand, resulting in a one-handed sign with the same meaning. Conversely, some one-handed signs are observed being produced with both hands having the same handshape and the same or mirror movement. Again, two different forms convey the same meaning. This type of variation is unique in signed languages as they have two articulators.

Table 2.1 lists the possible phonological variability in signed language and their analogical counterparts in spoken language. Phonological variation in sign language can be seen in the production of the compositional units of signs like handshape, location, orientation, movement, non-manual feature, and number of articulators. Early studies of phonological variation in sign language were small in scale and were mainly conducted in the US, i.e., the study of ASL. These studies investigated the variation in number of articulators (Battison, 1974; Woodward \& De Santis, 1977), location (DeSantis, 1977; Lucas, 1995; Woodward, Erting, \& Oliver, 1976), and handshape (Battison, Markowicz, \& Woodward, 1975; Hoopes, 1998; Metzger, 1993).

\begin{tabular}{lll}
\hline \multirow{2}{*}{ Variable unit } & \multicolumn{2}{c}{ Example } \\
\cline { 2 - 3 } Features of individual segments & Signed language & Spoken language \\
& change in location, & final consonant devoicing, \\
& movement, orientation, & vowel nasalization, vowel \\
& handshape in one or more & raising and lowering \\
Individual segments deleted or added & segments of a sign & \\
& hold deletion, movement & -t, d deletion, -s deletion, \\
Syllables (i.e., groups of segments) & first or second element of a & epenthetic vowels and \\
added or deleted & compound deleted & consonants \\
Part of segment, segments, or syllables & metathesis & metathesis \\
rearranged & & \\
\hline Source: Lucas et al. (2001, p. 25) & &
\end{tabular}

Table 2.1: Phonological variability in signed and spoken languages 
The results suggested that these phonological variables were influenced by both linguistic and social constraints. For example, symmetrical signs (both hands have the same handshape and the same or mirror movement) were more likely to delete the subordinate hand ("weak drop"); region and ethnicity had effects on both the choice of one-handed or two-handed forms and the place of articulation of certain signs. Another early study on BSL found that "weak drop" was more frequent in signs with unmarked handshapes, such as the B-handshape fact that some one-handed signs were formerly produced with two hands, Deuchar (1984) suggested that this variation of "weak drop" may indicate language change in progress in BSL.

Although the early studies have suggested the effect of extra-linguistic factors and other internal constraints in phonological variation, the role of the immediate phonological environment was reported even before the first large scale variation study was completed. For instance, Liddell and Johnson (1989) described the metathesis of DEAF (the production of chinto-ear non-citation form) and the change of handshape of the sign ME (PRO.1) as the outcome of phonological assimilation with the contiguous signs. That is, if DEAF was immediately preceded by a sign in lower areas (at or below chin), it would appear as chin-to-ear rather than ear-to-

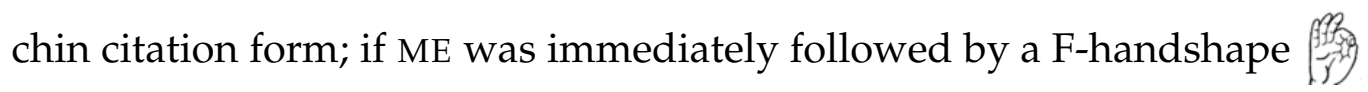
sign like CURIOUS, it would be made with a F-handshape rather than a 1-handshape citation form. A recent empirical study of phonological variation in BSL, together with the previous studies in ASL, Auslan, and NZSL (to be elucidated in the next two sections), showed that immediate phonological environment was only part of the explanation, other internal and external constraints were conditioning the variant choice of signers as 
well.

Following the ASL study (Lucas et al., 2001), Fenlon, Schembri, Rentelis, and Cormier (2013) examined phonological variation in BSL signs produced with a 1-handshape. Like the results of the ASL study, they found that both the surrounding phonological environment and grammatical function were significant linguistic factors. They further suggested that indexicality (i.e., pointing signs like pronouns) and lexical frequency were also important factors, and these two factor groups interacted with grammatical category. Since the three factor groups were non-orthogonal, the researchers combined grammatical category and indexicality for analysis and conducted a separate analysis with lexical frequency. The results showed that pronouns (i.e., indexical signs) and highly frequent signs (all indexical signs) strongly favoured handshape variation in two separate analyses. In other words, it was not possible to tease apart the effects of indexicality and lexical frequency. Unlike the ASL study where several social factors were found to be significant, region was the only social constraint reported as significant in the BSL study. As suggested by the researchers, this discrepancy may reflect the societal, historical and attitudinal differences in the two deaf communities. They also noted an important difference in their methodological approaches - the BSL study modelled the variation using mixed effects, while the ASL one used only fixed effects. As Paolillo (2013) notes, employing mixed-effects model may lead to possible Type II error when investigating the main effects, especially when the data may be sparse. In this case, there were just ten tokens or less from each of the 211 signers.

In McCaskill, Lucas, Bayley, and Hill (2011), they explored the nature 
of Black ASL as an ASL dialect. Like the earlier ASL study, they examined location lowering as one of the phonological features. They coded 877 tokens from both interview and conversation data collected from southern Black ASL signers in different settings. Their results showed similarities with the previous ASL study, yet with some differences. As in the earlier study, grammatical function was found to be the most significant linguistic constraint, but the order of factors differed. Other linguistic factors were preceding location and contact, in which preceding contact with the body favoured non-citation forms and no contact in preceding head location disfavoured them. For social factors, only region and age were found to be significant. Texas signers and those aged 35 and younger preferred the lowered variant. The differences may be due to both social and diachronic factors, as the two studies were conducted at different times.

The last two sections have introduced some fundamental findings in variation in signed languages. This sets the scene for introducing in detail the three foundational studies on which this thesis is based.

\subsubsection{The ASL study}

The first quantitative study of sociolinguistic variation was carried out in the US in the 1990s (Lucas et al., 2001, 2002). This study investigated variation in ASL at the lexical, phonological, and morphosyntactic levels. The data collected included free conversation, interviews, and lexical elicitation. At the lexical level, the signs for 34 concepts were elicited for examination. At the phonological level, two kinds of variables were analyzed: handshape and location. At the morphosyntactic level, null pronoun (sub- 
ject deletion) variation was investigated. Since the handshape variable has been mentioned in the previous section and null pronoun is irrelevant to the current project, they are not elucidated here.

In Lucas et al. (2001), a total of 207 ASL signers were recruited from seven sites, representing major areas of the country. Based on the history in language policy in deaf education, participants of three different age groups were recruited: 15 to 25, 26 to 54, and 55 and above. Participants in the first group (i.e., the youngest group) were educated at a point in history when awareness of the status of ASL had increased. The medium of instruction in schools at that time was Total Communication or ASL. ${ }^{9}$ Participants in the second group witnessed change in attitudes towards ASL, and experienced oralism or the change from oralism to Total Communication. The older participants in the third group were largely educated in oral residential schools. During that period of time, though the use of ASL was tolerated in the dormitories, it was forbidden in the classroom. The sample recruited a balanced number of males and females. This study also investigated the effects of language background, ethnicity, and social class. Hence, the sample was also composed of native and near-native ASL users, Caucasians and African Americans, and signers from working class and middle class.

The lexical elicitation task was done by showing a set of 34 stimuli (mostly pictures with some fingerspelling) to the informants. The selec-

\footnotetext{
${ }^{9}$ Total Communication is a teaching philosophy in deaf education introduced in the late 1960s, which suggested using all possible language modes, gestures, signs, speech, lip-reading, fingerspelling, reading and writing in educating deaf children. In practice, it became a teaching method that employed the simultaneous use of speech and signing (Baker \& Knight, 1998).
} 
tion of these 34 concepts was driven from earlier work on lexical variation in ASL, in which 1,200 sign variants for 130 lexical items were collected across the US (Shroyer \& Shroyer, 1984). The analysis of the 34 lexemes was concentrated on three concerns. The first concern was the relative proportion of lexical variants to phonological variants within each lexical item. The second one inquired into the functional and semantic patterns in variation if any. The last was concerned with whether there was any evidence of language change in progress, whether related to phonological constraints or lexical innovation.

Lexical variants referred to those that were unrelated to each other phonologically, whereas phonological variants shared many phonological features and differed in only one feature in most cases. The data suggested that the lexical items with the highest number of phonological variants were those most frequently used and most widespread across the country. However, the lack of statistical testing has left this association unvalidated. With regard to the amount of variation across the different syntactic categories, the average number of distinct variants of verbs, adverbs, and predicates (the combination of verbs and adverbs) were 8.7, 11, and 9.5 respectively. They were all notably higher than the average of 4 for nouns. On the side of semantic patterns, only the domains of food and animal were compared. The food average of 5.7 was higher than that of the animal signs which was 4 . Considering the number of possible lexemes that showed variation (at least 130 as mentioned earlier), these generalisations derived from 34 concepts appear to be inconclusive.

Concerning language change in progress, the researchers selected four signs to exemplify changes connected to location (from head to hand for 
RABBIT), handedness (from two-handed to one-handed for DEER), assimilation (from unassimilated non-dominant hand to assimilated for TOMATO), and compound form (from compound to simplex form for SNOW). Despite the varying stages of change observed, they described the evidence as "the usual and natural unfolding of phonological processes, of phonological variation followed by change, very similar to what we see in spoken languages" (Lucas et al., 2001, p. 191). At one extreme of the stages of change, two country signs: AFRICA and JAPAN, were used to demonstrate the completion of lexical innovation.

In relating the lexical variation to social factors, 27 out of the 34 concepts were found to have shared variants across all regions and some sites had unique variants not used in other sites. This high number of shared lexical forms across all sites was not surprising due to the fact that all residential schools had some connection with the American School for the Deaf, the first deaf school in the US. For other factor groups: age, gender, language background, social class, and ethnicity, less than one-third of the items had shared forms within each factor group. In other words, there were variants unique to each of the three age groups, female and male signers, ASL-family and non-ASL family signers, signers from middleclass and working class, African American and Caucasian signers. In particular, there were more unique forms used by non-ASL family signers and African American signers than their counterparts.

As for phonological variation, two location variables were examined: the sign DEAF and a class of signs produced at or near the forehead area represented by KNOW and FOR. The citation form of the ASL sign DEAF is produced by moving the index finger from ear-to-chin. There are two non- 
citation variants using the same handshape, one shows metathesis of the citation form, that is moving the index finger from chin-to-ear. The second one simply involves contact with the lower cheek. Four linguistic factor groups were coded: grammatical function (noun, adjective, predicate adjective, or compound), location of the preceding segment (high - at ear or above, middle - between ear and chin, or low - chin or below), location of the following segment (same as location of the preceding segment), and genre of text (conversation or narrative). The social factors coded were the same as in the lexical analysis.

Among the 1,618 tokens of DEAF analyzed, 31\% were produced in citation form, 55\% were chin-to-ear non-citation form and $14 \%$ were cheek contact. Taking the ear-to-chin citation form as the underlying form, the researchers assumed a model of rule ordering that explains the different variants of DEAF. First DEAF underwent metathesis and formed the chinto-ear form; then, movement-deletion created the contact-cheek form. Based on these assumptions, the initial analysis was citation form versus noncitation forms (collapsing chin-to-ear and contact-cheek into one category) and the second analysis was between the two non-citation forms, chin-toear and contact-cheek. The results of both VARBRUL analyses showed that grammatical function had the strongest effect, with predicate adjectives favouring the citation form and compound favouring the non-citation form, specifically the contact-cheek form. While no phonological constraint was found to be significant in the analysis of citation against noncitation forms, only location of the following sign significantly conditioned signers' choice between the two non-citation forms. With respect to the social factors, only age and region reached statistical significance in both analyses and interactions between the two were observed. For exam- 
ple, in some regions, younger and older signers were more likely to use non-citation forms than middle-aged signers; in one region, all signers favoured citation forms. The authors concluded that different variation patterns across the regions reflected the educational history of the deaf communities in the US.

In analyzing location drop in the class of signs produced at the forehead or temple, a total of 2,862 tokens were sampled. This class include nouns like FATHER, adjectives like DIZZY, interrogatives like WHY, prepositions like FOR, and verbs of thinking and perception such as THINK, BELIEVE, SEE. It is worth noting that BELIEVE is a lexicalized compound in which the first part begins at or near the forehead and the second part in a lower position, and SEE is articulated near eye level (Schembri et al., 2009). The researchers initially coded for 'impeded signing,' that is when the signers were uttering the target sign, their elbows or forearms were resting on a table, chair, or their laps. Their initial analysis showed that 'impeded signing' induced lowered signs as hypothesized. However, due to the fact that this kind of influence was subject to neither linguistic nor social factors, they eliminated the 268 tokens of impeded signing. This resulted in 2,594 tokens for multivariate analysis in VARBRUL.

There were eight linguistic factors coded for analysis and they were grammatical function of target sign (noun, adjective, verb, grammatical function words), preceding sign or pause, location of preceding segment (head or body), contact of preceding segment (no contact, contact with head or face, contact with body, contact with the other hand), following sign or pause, location of following segment (same as location of preceding segment), contact of following segment (same as contact of preced- 
ing segment), and genre of text (conversation or narrative). Social factors coded were the same as those for lexical analysis.

The non-citation variants were found to be more common than the citation forms, with the former accounting for $53 \%$ of the tokens and the latter $47 \%$. Results showed that three out of the eight linguistic factors were significant at the 0.05 level, namely, grammatical function, preceding location and following contact. Grammatical function words favoured noncitation forms with a factor weight of 0.581 , preceding location at head and following segments with contact disfavour it with factor weights of 0.463 and 0.466 respectively. Considering social factors, age, gender, region, language background and ethnicity by social class were found to have significant effects on the location of target signs. Older signers (aged 55 or above), women, rural signers (signers from Washington and Virginia), signers with deaf parents, and African Americans all disfavoured non-citation forms. In contrast, younger signers (aged 15 to 25) showed the highest factor weight (0.602) in favour of the non-citation variants, other significant factors favouring non-citation forms were if the signer was Caucasian or male.

\subsubsection{The Auslan and NZSL studies}

Since Auslan and NZSL are closely related languages of the BSL family (Johnston, 2003) and some parts of the results of the two studies were reported together, I delineate them here in one section. Modelled on the ASL study (Lucas et al., 2001), the Auslan study and the NZSL study were conducted in the 2000s, with the latter following the former about a year later 
(Schembri et al., 2009; McKee \& McKee, 2011). Like the ASL study, three types of data were collected: free conversation, informal interview, and lexical elicitation. ${ }^{10}$ Both studies had investigated the location drop variable (Schembri et al., 2009) and variable subject presence (McKee, Schembri, McKee, \& Johnston, 2011) based on conversation and interview data. Again, the syntactic variable is not presented here because of its irrelevance to the current study. McKee and McKee (2011) also reported their results in examining lexical variation in NZSL from the lexical elicitation data. I first describe the findings and results of location drop in both studies, then present the analysis of lexical variation in NZSL.

Both Auslan and NZSL studies employed the same data collection procedures. Participants were recruited through deaf contact persons from five sites across Australia and New Zealand. For the Auslan study, 70 groups of two to five participants were convened with a total of 205 participants and 147 of them were interviewed; whereas for the NZSL study, 55 groups of two to four giving a total of 138 participants and 69 of them were interviewed. Following the ASL study, the class of signs articulated at or near forehead in their citation forms were investigated. Examples of these signs in both Auslan and NZSL were THINK and NAME. Unlike the ASL study, the researchers excluded signs made lower than the eyebrow and lexicalized compound signs in which the second part was made at a lower place like ASL sign BELIEVE. Target signs produced above or on the eyebrow ridge were coded as citation form, while those below were coded as non-citation form. Both studies included tokens coded from the con-

\footnotetext{
${ }^{10}$ In the Auslan study, another two types of data were also collected: "an interview about the participants' sign names" and "two narratives" (Schembri \& Johnston, 2004).
} 
versation and interview data in order to test if any situational differences may occur.

Linguistic factors coded were grammatical function, preceding and following phonological environment, including location of the preceding and following signs, contact of the preceding and following signs, change in hand dominance before and/or after the target sign, pause before or after the target sign. In view of the recent research about the role of word frequency in phonological variation and change (Bybee, 2002; Dinkin, 2008; Phillips, 1984, cited in Schembri et al., 2009), the researchers also coded for lexical frequency. The ten most frequent signs in the list accounted for over $70 \%$ of the tokens in both Auslan and NZSL datasets. These top ten were coded as high frequency signs and the others were coded as low frequency. Their coding was supported by the findings of the Wellington Corpus of NZSL (McKee \& Kennedy, 1999; McKee \& Kennedy, 2006). This sign frequency factor was not considered in the ASL study. The Auslan study had conducted an inter-rated reliability test in coding the above linguistic factors by two coders and achieved $93 \%$ consistency.

Social factors coded in both studies were age, gender, region, and language background. For ethnographic reasons, the coding of these factors was different in the two studies. As for age, the Auslan study had four groups: 15 to 30,31 to 50,51 to 70,71 or above; the NZSL had three groups: 15 to 39,40 to 64,65 or above. The different age divisions in the two studies reflected differences in changes to deaf education and language policy in the two countries. The NZSL study further collapsed the five sites where the data was collected into three regions: north, central, and south. While the Auslan study also coded for social class (working class or mid- 
dle class), the NZSL study coded for ethnicity (Māori or Pākehā), but not social class because the socioeconomic status of New Zealand deaf people is generally homogeneous due to limited educational opportunities.

A total of 2667 tokens from 205 Auslan signers and 2096 tokens from 138 NZSL signers were analyzed using GoldVarb. ${ }^{11}$ More citation forms (55\% in the Auslan data and $57 \%$ in the NZSL data) than non-citation forms were found in the two studies, while the opposite was true for the ASL study where citation forms accounted for $47 \%$ and non-citation forms accounted for $53 \%$ of the corpus. With regard to linguistic factors, the results of the two studies were very similar. Four factors related to the immediate phonological environment were found to be significant and they were preceding location, following location, following sign or pause, and preceding contact. However, the most significant linguistic factor was the interaction between grammatical function and frequency of the target sign. Both studies revealed that high frequency verbs favoured non-citation forms with factor weights of 0.577 and 0.566 in the Auslan study and the NZSL study respectively. To conclude, linguistic factors that favoured location drop (non-citation forms) in both Auslan and NZSL are: (1) highly frequent verbs; (2) signs that were preceded or followed by signs produced in the body region; (3) signs that were followed by a pause; (4) signs that were preceded by signs making no contact with the body.

On the other hand, the two languages showed different patterns with respect to social factors, though with some underlying similarities. Age,

\footnotetext{
${ }^{11}$ A later version of VARBRUL, which was used in the ASL study mentioned in the previous section.
} 
region and gender were found to be significant in the Auslan data, and region, ethnicity, gender and language background were significant in the NZSL data. Both studies showed that large urban communities and females favoured the use of lowered variants. While younger Auslan signers (under 51 years old) tended to use more non-citation forms, age was surprisingly not a significant factor in the NZSL study. Language background showed an effect in the NZSL data where native signers favoured lowering, but not in the Auslan data. However, as the New Zealand native signer sample size was rather small, the researchers advised that this finding should be interpreted with caution. Lastly, Māori signers preferred citation forms, but Pākehā signers did not.

Further comparison among the results of the three studies will be discussed in Chapters 5 to 7 when the results of the current study are presented. I now turn to the other part of the NZSL study: lexical variation.

In examining lexical variation in NZSL (McKee \& McKee, 2011), 80 concepts were elicited using flash cards of pictures or English words. Social factors explored were age, region, gender and ethnicity. As with the location drop analysis, participants were grouped into three age groups: 18 to 39,40 to 64,65 or above. As mentioned earlier, the age groupings were based on changes in signers' experience with formal education. Those in the youngest group were exposed to the Australasian Signed English (ASE) in schools when the Total Communication approach was introduced in 1979. Before 1979, the oralist approach was adopted, and older signers were expected to use more mouthing, more limited sign vocabulary, and minimal use of fingerspelling. The import of ASE impacted on NZSL such that 'modern' variants were added to the lexicon. 
Again, the five sites of data collection were collapsed into three regions to investigate regional variation across north, central, and south. All informants had acquired NZSL before the age of twelve, with 91\% having acquired it before seven and this included some native signers (the proportion of native signers reflects the population profile), but language background was not analyzed as a factor in McKee \& McKee (2011).

Cross-tabulation of frequency of lexical variants by social factors was used to reveal the distribution patterns. A total of 249 distinct variants were recorded in 11,040 tokens. Only one concept (i.e., CHRISTCHURCH) showed no variation. Over $70 \%$ of the eighty lexical items had two or three variants, one-fourth of them had four to five variants, and two concepts had six variants. Unlike the ASL study, the most frequently used and widely distributed (across regions) variants did not have the highest number of subvariants in NZSL; and nouns rather than verbs and adverbs showed more variation in this dataset.

In terms of social factors, age group was the most prominent amongst the four examined. Young signers used more 'modern' forms, while older signer favoured 'early' ones. Complete change was observed in concepts like DEAF and FRIDAY, where no young signers use the 'early' variants. Moreover, semantic change in some early variants was identified. While older signers used mouthing together with generic signs to distinguish meaning in semantic classes like colours and months, younger signers used more different signs, reserving the generic signs to refer to the category and occasionally some terms within the category.

Regional variation was realized in the proportion of variants used rather than unique regional variants. Similar to the ASL study, considerable com- 
monality was observed across the three regions in the most frequently used variants. Yet, there was substantial contrast between signers from the north and the south. Unsurprisingly, certain concepts did capture gender and ethnicity differences. They were sports signs (presumably the men were more interested in sports than women in general) and Māori related concepts respectively. The researchers commented that a decontextualized elicitation task is not an effective way to investigate gender variation, as such a variation is more likely to manifest itself in discourse. Notwithstanding, gender appeared to be one of the significant factors when they analyzed the use of 'less frequent' versus 'more frequent' variants.

Multivariate analysis was conducted to confirm the observed lexical leveling in the data. All tokens were coded as either frequent or nonfrequent based on their occurrence in the data. The former mainly consisted of 'modern' forms but were reasonably standardised, whereas the latter included 'early' and highly innovative forms. By using the statistics package GoldVarb, their analysis showed that age, region, and gender were all significant factors in predicting the use of frequent variants. Younger and middle-aged, northern, and female signers tended to produce more frequent variants, which the researchers interpreted as an indication of lexical leveling and a progressive standardization. They surmised that this reflected the effect of the top-down intervention of language policy in deaf education. Both findings related to age and region were parallel to those found in the studies of New Zealand English.

Indeed, the results of the lexical variation study mirrored an earlier pilot study on numeral variation in NZSL (McKee, McKee, \& Major, 2011). The numerals one to twenty were examined: half of them displayed high 
consistency and the other half showed greater variation. This variation was correlated to social factors, and age was found to be the strongest social factor, followed by region and then gender. Again, there was an indication of increasing standardization among younger signers in terms of the number lexicon. Some formerly known two-handed forms were absent in the dataset and an age-related shift from traditional two-handed to one-handed forms was observed. This echoed the findings of the BSL study mentioned earlier (Stamp et al., 2015). The researchers concluded that it was the result of both internal change (as suggested in the BSL study) and the adoption of ASE variants.

\subsection{Variation and documentation}

Linguistic research which involves the collection of primary language data, regardless of its aim, can be viewed as a form of language documentation (Himmelmann, 1998, p. 165). As Woodbury (2011) said, “Language documentation is the creation, annotation, preservation and dissemination of transparent records of a language" (p. 159). Documentation of human languages has a long history. It has evolved as a response to endangered languages following a call for action to record and archive vanishing languages in the early 1990s (Hale et al., 1992). Work on language endangerment has posed substantial challenges, such as demands on fieldwork practices, to the traditional structuralist models of linguistics (Himmelmann, 2008). Over the past two decades, language documentation has developed as a subfield of linguistics, also known as 'documentary linguistics.' While different from descriptive linguistics, the two complement 
each other in terms of activities, goals and outcomes (Austin, 2010).

Certainly, not only endangered languages are in need of documentation and the conventions of language documentation can be applied to non-endangered languages as well (Austin, 2010; Himmelmann, 2008). While expanding the research targets to non-endangered languages, researchers have become aware of a lack of a consideration about social factors in this emerging field. This awareness has caused researchers to rethink their practice and explore the link between language documentation, sociolinguistics and language revitalization (Farfán \& Ramallo, 2010). In responding to new directions for research and action in the field, Grenoble (2013) advocated the following,

... that we should expand documentation to other understudied, pre-shift speech communities - is based on the belief that the description and documentation of languages and linguistic structures is itself an incomplete and insufficient enterprise; our real goal should be a description and documentation of language ecologies. That is, we need to study languages as they are culturally and socially situated, in a full social context of production and use (p. 54).

We need to rethink some of our research targets and document not just ancestral languages but languages in the process of shift; to work with all kinds and varieties of speakers, at all levels of proficiency, and in all kinds of situations (p. 55).

This echoes Woodbury's (2011) claim that documentation should not focus only on fluent language users, but also be the study of "semi-speakers" 
and "of the variation in communities undergoing rapid language shift" ( $p$. 180). Indeed, variation should be part of the agenda in any language documentation project, as "no documentation can be complete without a study of variation" (Grenoble, 2010, p. 82). In actual practice, both goals of documentation and variation study can be achieved together and impact the local community in a positive way (Meyerhoff, 2015).

In regard to sign language documentation, various projects started in the 2000s. As Schembri (2010) noted, some projects aimed to create a sign language corpus, such as the Corpus NGT,12 while some had the dual goals of corpus creation and sociolinguistic research on variation and change like the BSL Corpus Project. ${ }^{13}$ In fact, the study of sign language variation requires the collection of natural language data from a representative sample of the deaf community, which results in the creation of a corpus. Hence, in the current study, a corpus of HKSL is created. In spite of the small scale of the corpus, it is important to take a 'snapshot' of this language that is changing rapidly as a result of the educational change that has happened in the past decade (which will be explained in the next chapter).

\footnotetext{
${ }^{12}$ Corpus NGT: www.ru.nl/corpusngtuk

${ }^{13}$ BSL Corpus Project: www.bslcorpusproject.org
} 


\subsection{Chapter summary}

In this chapter, we have seen that both lexical and phonological variation are common in signed languages. As with spoken languages, some of the variation is constrained by linguistic factors and some by social factors. Major social factors like age, region, gender, social class, and ethnicity found in oral languages are also contributing factors to variation in signed languages. Besides, having double articulators, language background and school are unique factors observed in signed languages. I have also detailed the studies in ASL, Auslan and NZSL, describing their methods and findings. Lastly, I have given an account of variation and documentation.

The literature review has provided the background information about sociolinguistic variation in signed languages and has recounted the exemplars of the current study. While the literature review is important in forming the present research, understanding the context of the language under study is equally important. In the chapter which follows, I delineate the sociohistorical context for variation in HKSL. 


\section{Chapter 3}

\section{Sociohistorical context for HKSL variation}

\subsection{Overview}

This chapter provides the sociohistorical context for variation in HKSL: the history of deaf education and the social conditions that make the presence of variants possible. Research on deaf history in Hong Kong is scarce. The chapter is mainly based on the work done by Hong Kong Society for the Deaf (1987), Sze, Lo, Lo, and Chu (2011, 2013), a recent thesis on signs used in two of the oral schools by Yu (2014), and some school publications. I also draw on narratives from my interview data and informal interviews with two teachers of the deaf. Some information comes from my own experience and connection with the Hong Kong deaf community. In Section 3.2, I begin with an overview of deaf education in Hong Kong since the establishment of the first deaf school in 1935. I then examine the language 
policy and language use in different deaf schools over the past decades in Section 3.3. A simplified picture of how different school varieties have mingled to form the modern form of HKSL and how HKSL is used in the deaf community is depicted in Section 3.4. Section 3.5 describes the language contact situation in the community. Lastly, Section 3.6 gives a summary of this chapter.

\subsection{History of deaf education in Hong Kong}

Although Hong Kong was a British colony for over 150 years, there is hardly any trace of British Sign Language (BSL) in the modern form of HKSL. Rather, the Shanghai variety of Chinese Sign Language (CSL) has influenced HKSL deeply through education in the late 1940s to the mid1970s. ${ }^{1}$ In their recent study, Sze et al. (2013) elucidate the role of the first two deaf schools in HKSL development. They suggest that "a signing variety developed among the residential students" (p. 175) of the first deaf school, which was established in 1935, long before the second deaf school introduced the Shanghai variety of CSL to its students in 1948. They further conclude that the modern form of HKSL is a mixture of this Shanghai variety and what they refer to as the locally developed varieties (Sze et al., 2013). Figure 3.1 below shows an overview of deaf education in Hong Kong over the past decades and how the Shanghai signs disseminated

\footnotetext{
${ }^{1}$ I use Shanghai variety throughout the thesis instead of Nanjing/Shanghai variety, unless quotes from Sze et al.'s study (2013) are used. This is because the Shanghai variety is one of the two main varieties in CSL, Nanjing and Shanghai are geographically close and their signing are quite similar.
} 
to other deaf schools through teachers and students of the second deaf school.

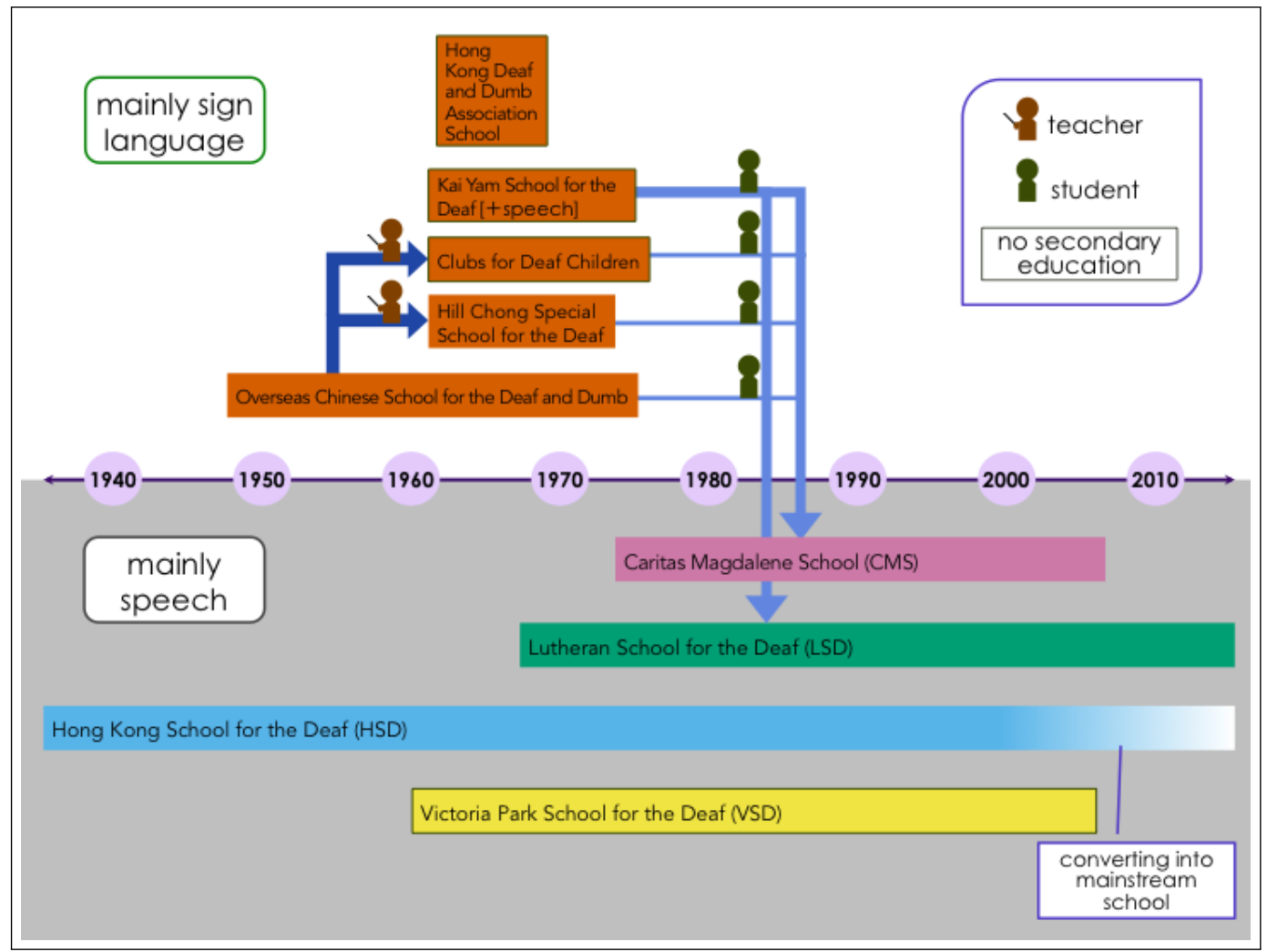

Figure 3.1: Overview of deaf education in Hong Kong (See Section 3.3 for details)

Figure 3.1 shows that deaf education in Hong Kong has been predominantly oralist, except for the period from the late 1940s to the mid-1970s. According to Sze et al. (2013), the Education Department set up a Special School Section in 1960, and took over the responsibility of educating deaf children, which was under the Social Welfare Department. The Education Department also invited an audiology professor from Gallaudet University (College at that time) to advise on deaf education. The consultancy report stressed the needs for early intervention, audiological services, and 
verbal communication (Frisina, 1963, cited in Sze et al., 2013). This led to a policy and attitude change towards an auditory/oral approach and inclusive education in the early 1960s. As a result, all manual (i.e., signing) schools and clubs for deaf children were closed down in the mid-1970s and the enrolment in deaf schools has decreased gradually every year. In the 2000s, two oral deaf schools have closed down; only one deaf school remains, whereas another one has converted to a mainstream school and is phasing out their deaf students.

Despite the speech-only policy from 1970s to 2000s, limited signs were used in some classrooms and signing was tolerated during recess and in the dormitories in schools. Like other parts of the world, schools for the deaf provide optimal conditions for the development of sign language, deaf identity, and deaf community, particularly residential schools (Senghas, Senghas, \& Pyers, 2005; Winzer, 1993; Woll, Sutton-Spence, \& Elton, 2001). Some lexical variation observed nowadays is anecdotally attributed to the signs created in different schools during this period of oralism. As one of the older informants who went to a club for deaf children (which used sign language as the medium of instruction) commented, "It was better in the past. There was only one sign language... It was about 1980, the signing changed. People from different deaf schools signed differently..." (LWF, December 20, 2013). 


\subsection{Deaf schools in Hong Kong from mid-1930s to 2000 s}

In this section, I give a brief description of each school (or a group of institutions in the case of the old manual schools/deaf clubs). This is because knowing the schooling background of the participants may help us better understand the variation patterns found in the analysis reported in later chapters. By including the participants' and the teachers' views and experience, I wish to give a more complete picture. As indicated in the previous section, deaf education in Hong Kong was very much either manual or oral before the mid-1970s and oral only afterwards. No artificial sign system such as Signed Chinese was invented or adopted formally in any of the deaf schools during the past 80 years. ${ }^{2}$ Figure 3.2 shows the rough location of the four oral schools (in black) and clubs for deaf children (in grey).

\footnotetext{
${ }^{2}$ Similar to the manually coded English system in the United States, Signed Chinese is an artificial signed system created by educators with an aim to teach deaf students Chinese. Different Signed Chinese systems have been invented in China and Taiwan, and have been used in deaf education (Lytle, Johnson, \& Hui, 2005; Smith, 2005).
} 


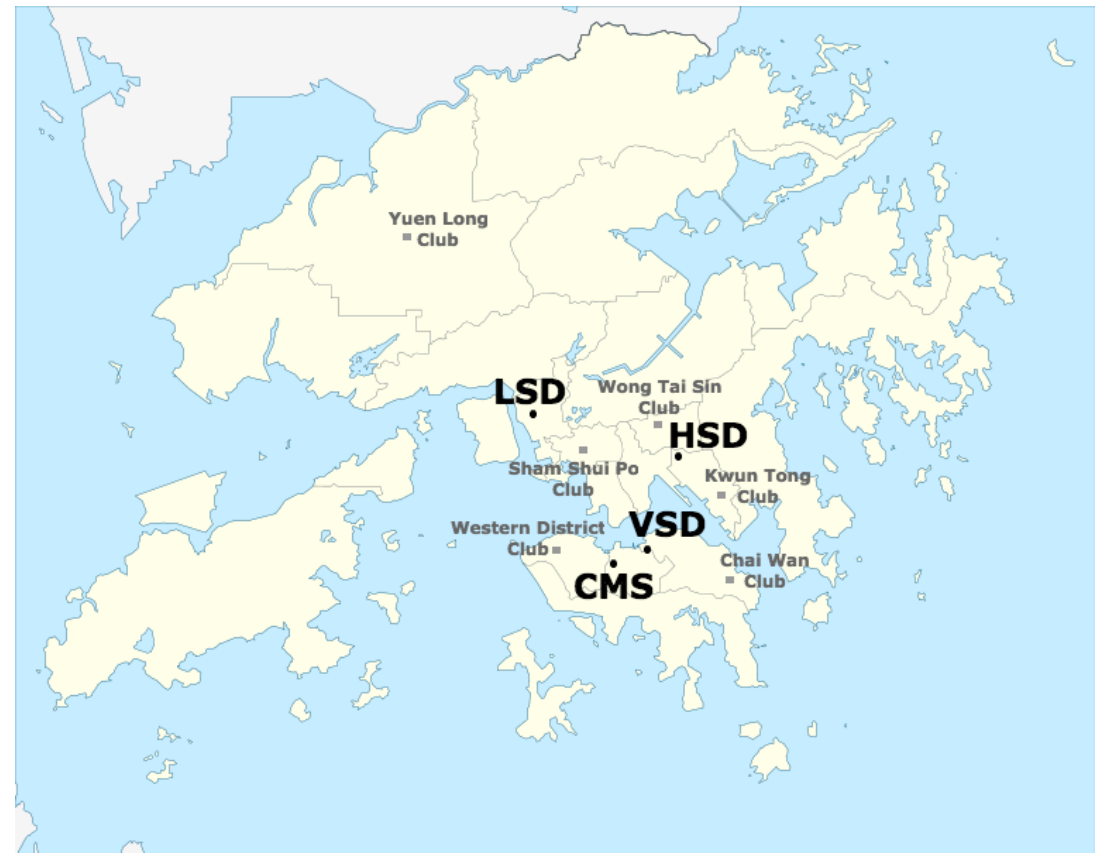

Figure 3.2: Hong Kong map showing the location of oral schools and clubs for deaf children

\subsubsection{Old manual schools (OMS)}

In the current study, I use Old Manual Schools (OMS) to refer to the four private manual schools and six clubs for deaf children run by the Social Welfare Department in the history of Hong Kong deaf education. Most of them operated until the mid-1970s (see orange boxes in Figure 3.1 on page 49). Table 3.1 gives a brief profile of them. Except Kai Yum School for the Deaf, which used speech together with signs, all other schools and clubs used sign language as the medium of instruction. 


\begin{tabular}{|c|c|c|}
\hline Name of school & Duration & Education offered \\
\hline Overseas Chinese School for the Deaf and Dumb & 1948 to $1975 / 76$ & Kindergarten to Secondary 3 \\
\hline \multicolumn{3}{|l|}{ [Kowloon branch opened in 1956] } \\
\hline Hill Chong Special School for the Deaf & 1961 to 1974 & Kindergarten to Secondary 2 \\
\hline Kai Yum School for the Deaf & 1961 to $1975 / 76$ & Kindergarten to Primary 6 \\
\hline Hong Kong Deaf and Dumb Association School & 1962 to 1969 & Primary only \\
\hline Clubs for Deaf children & & Primary only \\
\hline 1. Wong Tai Sin & 1960 to 1977 & \\
\hline 2. Western District & 1960 to 1975 & \\
\hline 3. Sham Shui Po & 1961 to 1977 & \\
\hline 4. Chai Wan & 1964 to 1972 & \\
\hline 5. Kwun Tong & 1966 to 1975 & \\
\hline 6. Yuen Long & 1970 to 1977 & \\
\hline
\end{tabular}

Table 3.1: Private deaf schools and clubs for deaf children before 1980

The second deaf school in Hong Kong, the Overseas Chinese School for the Deaf and Dumb (OCSD), was established by a deaf couple from China in 1948. The principal was originally from Nanjing and graduated from a deaf school in Shanghai (上海福啞學校) (Sze et al., 2011). Hence, the Shanghai variety of CSL was brought into use in OCSD. The American fingerspelled alphabet was used in the Shanghai deaf school that the principal went to (Yi, 2008). Therefore, it is possible that the American fingerspelled alphabet was disseminated in Hong Kong at that time. The sign language used in OCSD was spread to other private deaf schools and clubs for deaf children through their teachers and graduates in the 1960s; and later, through their students to oral deaf schools when the signing schools and clubs closed down in the 1970s (see Figure 3.1 on page 49). The role of OCSD and the language they used played a prominent part in Hong Kong deaf education and the development of HKSL. As Sze et al. (2013) remark, "the large student population, the strong signing tradition, and the high level of the students' signing proficiency partly explain why 
the Nanjing/Shanghai variety introduced by the OCSD became so influential in the subsequent development of HKSL" (p. 170).

While some teachers of OCSD went to teach at Hill Chong Special School for the Deaf and the clubs for deaf children and brought along the signing variety used in OCSD, not much information about the other two private schools (Kai Yum School for the Deaf and Hong Kong Deaf and Dumb Association School) is known. According to the documents collected by Sze and her colleagues, there were only an average of seventy students enrolled in Kai Yam School and Hong Kong Deaf and Dumb Association School annually throughout their operating years in the 1960s, as opposed to an average of over three hundred enrolment in OCSD, clubs for deaf children, and Hill Chong School for the Deaf (Sze et al., 2013). ${ }^{3}$ Moreover, one of their informants informed them "the signing variety at the Hong Kong Deaf and Dumb Association School was similar to the Shanghai signs" (p. 173). Nevertheless, we can assume that most of the deaf people who studied at OMS learnt the Shanghai variety of CSL. These deaf people, most of them aged over 60 now, and their signing have had a major influence on the development of the modern form of HKSL, a variety closely related to the Shanghai signs (Woodward, 1993).

It is interesting to note that the two-handed fingerspelled alphabet used in BSL, Australian Sign Language (Auslan), and New Zealand Sign Language (NZSL) was found being used for student's name signs in clubs for

\footnotetext{
${ }^{3}$ These documents included the Social Welfare Department's annual reports (1948-1971), the Education Department's annual reports (1961-1972), the Hong Kong government's annual reports (1954-1964), and the Hong Kong Annual Digest of Statistics (1980-2009).
} 
deaf children. Three of the participants from the club for deaf children in Yuen Long told me that name signs were assigned to them by their teachers according to the sequence when they entered the club. The first child in was $\mathrm{A}$, second was $\mathrm{B}$, and so on. One of them still uses this name sign now. This may suggest that there were some other sources for the signing used in this club for deaf children or possibly in other clubs as well. More research is needed to explore this possible source.

\subsubsection{Hong Kong School for the Deaf (HSD)}

Established in 1935, the Hong Kong School for the Deaf (HSD) was the first school providing formal education for deaf children in Hong Kong. It was set up by Christian missionaries, and the first principal was trained in Chefoo school for the deaf in China (Hong Kong School for the Deaf, 1995). Same as the schools established in China by missionaries with the same training background, HSD adopted the oral approach from its opening. Located at Kowloon East since 1948, HSD was first run privately and received a government subsidy starting in 1961 (Hong Kong School for the Deaf, 1995). In 2004, HSD was converted to a mainstream school and renamed as Chun Tok School. ${ }^{4}$ Only hearing students are enrolled and the remaining deaf students are phasing out now. At the time of the fieldwork for the current study, i.e., early 2014, only a few Form 4 to Form 6 deaf

\footnotetext{
${ }^{4}$ The Chinese name of HSD was 真鐸啓喑 and its transliteration is 'Chun Tok Kai Yum.' The last two characters literally mean 'open-mute.' Therefore, they were removed from its name when HSD was converted to a mainstream school and their English name was transliterated from this new Chinese name.
} 
students remained in HSD. ${ }^{5}$

Among all the deaf schools, HSD was regarded as an elite school, in the eyes of both educators and deaf adults. Some older participants (aged 50 or above) recalled their experience of being rejected by the principal of HSD when their parents took them to the school for interview. The reasons for being rejected might be that the deaf child was too old and had no or poor speech, or the child had learnt to use signs in a manual school or in a club for deaf children. One of the rare cases who got accepted in HSD was a participant who is now in his 50s. This participant went to HSD after having several years of education in the OCSD (one of the OMS, see Section 3.3.1 on page 52). He told me in the interview that he was referred to Victoria School for the Deaf (VSD, see Section 3.3.3 on page 58) for speech training, so that he could enter HSD.

It is believed that students who could continue their education after finishing their study in VSD would go to HSD for secondary education in the early years. This was because VSD was a companion school to HSD, and other deaf schools had not been established until the late 60s to early 70s. But in later years, only those who had good academic results would be recommended to HSD. As one of my friends in her thirties who went to VSD told me, most of her classmates would go to Caritas Magdalene School (CMS, see Section 3.3.4 on page 60) for secondary education, only a few top students would go to HSD. Likewise, when only HSD offered higher secondary education (Form 4 and Form 5) in the old

\footnotetext{
${ }^{5}$ In general, Form 4 to Form 6 students aged around 16 to 18 in mainstream schools. Students in deaf schools maybe slightly older and may have a wider age range.
} 
days, the other two oral schools would recommend a few students who demonstrated good academic performance to HSD to further their studies. It seems that HSD always had the privilege of selecting and accepting 'good students.' One of the teachers from Lutheran School for the Deaf (LSD, see Section 3.3.5 on page 62) told me, "according to the education policy, students should be allocated to the school near their home. However, if a deaf child is good, the child will be sent to HSD even though the child lives here." ${ }^{6}$ She further explained, "when the number of deaf children enrolling in deaf schools declined, we accepted all deaf children with multiple handicaps. But HSD refused to accept any of them" (Ms Tsang, February 19, 2014). 'Good student' here may refer to a deaf child of normal or above intelligence, who has demonstrated good academic performance in another deaf school (if transferred from another school), and has good family support to perform speech training at home (especially when the child was young). It is interesting to note that there were quite a number of deaf students with deaf parents from HSD, even though their first language was sign language (which opposed the school's strict oral policy). This may reflect that these children have better cognitive development than their peers with hearing parents when they entered school.

In spite of their strict aural-oral approach, signing was tolerated in recesses and the dormitory in HSD. ${ }^{7}$ As evidenced in Sze et al.'s study (2013), a signing variety developed among the residential students within

\footnotetext{
${ }^{6}$ She was in Yuen Long, a local place far away from HSD, at the time of the interview.

${ }^{7}$ All students of HSD were boarding from the opening of the school in 1935 to 1958, and probably until the mid-1960s. In 1968, about $73 \%$ of the students were boarding, whereas in 1978, 1985 and 1995, the percentage dropped to less than 8\%. (Hong Kong School for the Deaf, 1995, p. 83).
} 
13 years of its reopening in 1949 (p. 169). Certainly, this variety has passed from older students to newcomers over the years. It is possible that some teachers had learnt some signs from their students in the old days, as students of the 1970s and 1980s remembered some teachers secretly using signs in classroom when the principal was not around. One of the participants, who went to HSD in the 1950s, had worked as a workman in HSD for nearly 50 years after he finished his primary education there. Although he complained that the younger generation made up their own signs and he could hardly understand them, his role as a deaf adult in the school would definitely have helped in transmitting the HSD signing variety from generation to generation. Furthermore, there was a deaf teacher, who is also an alumna of HSD, and she taught arts at HSD for over ten years before her retirement. This would also have contributed to creating an environment providing adult models for students coming into HSD in later years.

\subsubsection{Victoria Park School for the Deaf (VSD)}

Due to the high demand for deaf education for those who could not afford to go to private school, VSD was set up in 1960 as a companion school to HSD. The principal of HSD was in charge of VSD when it was first established. In spite of that, VSD was located at Hong Kong Island, geographically not close to HSD. As the number of deaf children declined and many of them were mainstreamed in later decades, VSD closed down in 2006. Like the clubs for deaf children, VSD did not provide secondary education. Students who could continue their education after finishing their study in VSD would go to HSD for secondary education in the early 
years. After the establishment of CMS in 1973, an increasing number of the graduates from VSD went to CMS because they were geographically closer; both schools were on Hong Kong Island (see Figure 3.2 on page 52). As mentioned in the early section about HSD, only a small number of students with good academic results would be recommended to HSD. Among the 65 participants in the current study, eleven of them transferred from VSD to CMS whereas seven switched from VSD to HSD. Only one who went from VSD to CMS is over forty years old, the remaining ten are aged below forty. On the other hand, five out of the seven who went to HSD are aged forty or above, and the two younger ones have deaf parents. When the primary section in CMS closed in 1993, their students were transferred to VSD to continue their primary education before being promoted to the secondary section of CMS. In addition, some students of VSD who lived far away from the school had stayed in the dormitory of CMS from 1994, i.e., both primary students from VSD and CMS and secondary students of CMS were living in the same dormitory. These events all evidenced the close connection between VSD and CMS after the latter was established in 1970s.

As in HSD, speech was the main medium of instruction in VSD. However, those who had studied in VSD reported that limited signs were used by some teachers. Based on the interview data, all the teachers were hearing, no deaf teacher or worker was employed in VSD. The teachers used mainly speech, with very few simple signs in classrooms. Signs were usually used by more experienced teachers and they could only sign secretly when not being observed. Students used signs among themselves during recess and they learnt from seniors and peers. These narratives (and those about CMS described in the next section) affirmed those found in a recent 
research by Yu (2014) focusing on the signs used in VSD and CMS. Additionally, he found that some VSD teachers went to the Hong Kong Society for the Deaf to learn sign language (Yu, 2014, p. 32).

\subsubsection{Caritas Magdelene School for the Deaf (CMS)}

Formerly known as Canossa School for the Deaf, CMS was set up by Canossian Daughters of Charity in 1973. It was renamed in 1991 when Caritas Hong Kong, the official social service agency of the Catholic Church, took charge of the school. The school first provided kindergarten and primary education only. A year later in 1974, the junior secondary section and dormitory were opened and senior secondary education was offered in 1981 (Caritas Magdalene School, 2000). In 2007, CMS closed down for the same reason as VSD mentioned in the previous section.

When CMS first opened, it received many students from the signing schools and clubs for deaf children, which were closing down around the mid-1970s. One of my participants was among the first cohort in CMS. She was from the club for deaf children in Yuen Long and she told me all the younger students were moved to CMS while the older ones (probably Primary 4 or above) remained in the club. ${ }^{8}$ New students also included those who previously studied in hearing schools. She lived in the dormitory for three to four years and remembered the staff there learnt signs from students. Another informant who was transferred from OCSD to CMS in

\footnotetext{
${ }^{8}$ Nowadays, mainstream Primary 4 students are about 10 years old. But in the 1970s, children entered schools at a later age and the age range in the same grade was wider.
} 
1975 recalled teaching signs to both teachers and students in CMS. One informant who entered CMS at the age of five in 1975 told me he was impressed and excited when he saw students signing among themselves and some teachers knew signs too. But he was disappointed after a few years, as he said the teachers used more speech after an Australian expert came to visit the school. ${ }^{9}$

Apart from these anecdotes about the early years, most informants reported similar stories to those in VSD: all teachers were hearing, classroom teaching was mainly oral with limited signs, only a few experienced teachers used some signs. Nevertheless, the change in teaching method due to the change in education policy and philosophy when Caritas took over CMS was recounted by the informants in Yu's study. Yu (2014) noted, "Some experienced teachers who used to sign or adopt simultaneous communication teaching in CSD (Canossa School for the Deaf) were not allowed to do so anymore in the classroom in CMS" (p. 17). However, none of my participants from CMS mentioned this change. I also talked to a former CMS teacher, who taught there from 1974 to 2003, during my fieldwork in Hong Kong. She did not bring up this issue in our conversation. To her, oralism was the main policy over her 30 years of teaching in CMS. Despite the "no sign language allowed" order from the principal, she learnt signs from her students and used signs to communicate with her students. Nevertheless, she considered her signing to be very limited and according to her, most teachers did not know or only knew very few signs.

${ }^{9}$ There is no other information about the Australian expert this participant mentioned. 
It is very likely that the signs used in CMS were created and developed by its students based on the signs brought along by those who had studied in signing schools, i.e., the Shanghai variety. While signing was not sanctioned in classrooms, the dormitory allowed it to flourish. Besides the earlier anecdote about students teaching signs to the staff in dormitory when it first opened, a deaf caretaker was employed later in 1980s and she worked there until the school shut down in 2000s. In my interview data, one non-boarding informant expressed her feeling of amazement when she first saw the dorm social worker signing with her boarding schoolmates. Apparently, the language policy in the dormitory was different from the one adopted in the school.

\subsubsection{Lutheran School for the Deaf (LSD)}

Lutheran School for the Deaf (LSD) was established in 1968 and it is now the only deaf school in Hong Kong which offers education from Primary 1 to Form $6 .{ }^{10}$ The school was first located at Kowloon West (KLW), it was then moved to its current address at New Territories West (NTW) in 1990. Unlike the other three oral deaf schools, LSD has been allowing the use of signs even though they claim that they employ the oral approach. Moreover, LSD was the only school that has adopted cued speech since its early years. Cued speech is "a visual mode of communication that uses handshapes and placements in combination with the mouth movements of speech to make the phonemes of a spoken language look different from

\footnotetext{
${ }^{10}$ LSD previously provided up to Form 5 education. Due to the recent education reform in Hong Kong, secondary education is extended to Form 6.
} 
each other" (National Cued Speech Association, n.d.). This method was mainly used in the primary section, and it was phased out when the cuedspeech-trained teachers retired. While cued speech was being emphasized in primary classrooms with the limited use of signs, signing has been used together with speech in the secondary section over the past forty years. The interview data confirmed this brief summary of language use in LSD.

In addition, Total Communication is an approach that only LSD has employed and it is still emphasized in educating deaf children (Lutheran School for the Deaf, 2009). As mentioned in Chapter 2, Total Communication originated in the United States in the late 1960s. This philosophy simply means incorporating all possible ways to communicate with deaf children, including but not limited to speech, signs, listening, lip-reading, visual aids, written text, and (formerly) cued speech. Having said that, the use of signs in the classroom has depended largely on the teachers, their attitude towards sign language, and their signing skills. Hence, the quantity and quality of signs used in LSD have varied from teacher to teacher, and from time to time (this is also true in the other oral schools).

It seems that LSD has been the most tolerant among the four oral schools regarding the use of sign language. When I asked one of the LSD teachers, who taught there from 1980s to 2010s, about the origin of LSD sign language, she told the following story:

We all learnt from an old teacher called Miss Suen, Suen SauYing [transliterated from Cantonese]... When LSD was first opened, sign language was taught before they started teaching the curriculum. There was a group of teachers, who discussed and studied about signs. They drew pictures to teach vocab- 
ulary. The signing skills of that group of teachers were better [than the teachers now]...

As new teachers, we sought help from Miss Suen whenever we got stuck with signs. And I know that Miss Suen and others [teachers] had made a little book, full of drawings, which was used in LSD...

... In the primary section, apart from cued speech [cued speech trained teachers], there was a group of teachers, who taught sign language in the past, and they signed better than us [current teachers]... (Ms. Tsang, February 19, 2014)

In the interview, I then asked where the sign language used by that group of teachers came from. She replied, "From deaf people, I believe." (Ms. Tsang, February 19, 2014)

This account of historical events may need to be verified with more evidence. Yet, it suggests that the sign language used in LSD may not have solely been created by their deaf students in the early years, as in the case of HSD. There may be a possibility that some teachers (like the students) from the old signing schools had transferred to LSD. Another possible consideration is the role of the Lutheran Deaf Evening School as noted in the work of Sze and her colleagues. According to the information they collected, the Lutheran Church set up an evening school for the deaf in 1965, three years before LSD was founded, and one of the deaf graduates from Hill Chong Special School for the Deaf, who was then employed as a teacher there, switched to the Lutheran Deaf Evening School (Sze et al., 2013, p. 172). Though no information about the operation and closure of the evening school was reported in their work, it is very likely the two 
schools were associated.

However, there was a contradicting account told by one of my participants, who was enrolled in the second semester just after LSD's opening in 1968. He said the school was oral only, no sign was allowed when he first entered. The principal at that time was British and he wanted the students to focus on learning English. If the students were good, he would send them to England or Germany to study English. Later, this interviewee said, when a new student who already knew signs entered the school and taught other students signs, the principal was angry, he made no more plans to send any of them abroad, and he finally left Hong Kong. "All teachers did not know signs. Someone from Australia was invited to teach us cued speech," he added (CKW, January 14, 2014). Certainly, more research on the history about the sign language used (or taught as the teacher said) when LSD first opened is needed.

The openness in using sign language in the classroom and the adoption of cued speech made LSD unique among the oral schools. There is a deaf teacher who went to VSD and HSD, and graduated from Gallaudet University, teaching at LSD. He has been teaching since 1988. It is unsure how his signing may have impacted on the sign language use in the school. Furthermore, due to its location, there have been many immigrant children from China enrolled in LSD over the past decades. These children of various ages came from different parts of China with diverse linguistic backgrounds and they have added complexity to the language used in the school. 


\subsection{Language use in the deaf community}

During their time in school, deaf students did not have much time to socialize with deaf students from other schools. However, after their graduation, they had more opportunities to meet other deaf people with different schooling backgrounds when they went to deaf organizations or deaf events. The first deaf organization, Hong Kong Deaf and Dumb Association, was established by some deaf people in 1955. It also founded a primary school in the 1960s for a short period of time as mentioned earlier in Section 3.3.1. Later, the Y's Men's Centre for the Deaf was founded by the Chinese YMCA of Hong Kong in 1967, the Hong Kong Society for the Deaf was set up in 1968, and the Cheung Ching Lutheran Centre for the Disabled was launched in 1984. In 1976, a group of deaf people established the Hong Kong Association of the Deaf. While the first deaf organization seems to be fading out in the past decades, the other four deaf organizations have offered places and opportunities for deaf people with different schooling backgrounds to gather and socialize during this time. As most of the participants told me, their friends include their old classmates and those from other deaf schools.

Indeed, some of my participants told me they met deaf people from other deaf schools when they continued their education at the Vocational Training Centre or other educational programmes for deaf students who finished Form 5. While they noticed differences in their signing, most of them did not consider it a big problem and they could communicate with each other quite well. In other cases, deaf people continued their education in mainstream institutions where they were the only deaf person in class with no interpretation or note-taking services. While some endured 
and tried their best to finish their studies by seeking help from hearing classmates or teachers, some just gave up and looked for work.

Over the past fifteen years or so, more deaf related organizations have been set up. A special one is the Centre for Sign Linguistics and Deaf Studies (CSLDS), which was established in 2003, under the Department of Linguistics and Modern Languages in the Chinese University of Hong Kong (as mentioned in Chapter 1). The centre employs many deaf people to work on HKSL research and education programmes. Several of my participants work in the centre and have expressed that their views and perceptions about HKSL have changed since they entered CSLDS. Certainly, their now positive attitude towards the language and the knowledge they have learnt about the language would have disseminated to other deaf people who are not working in the centre through their networks.

Furthermore, the CSLDS deaf workers are more aware of natural signing and a more contact form of signing, which would incorporate more Chinese features such as mouthing and word order. When I requested my friends (who are working or have worked in CSLDS) to refer participants for the current study, some of them would ask me if I needed good signers. In fact, even if most members of the deaf community went to deaf school, their signing competence may vary. It is the reality that deaf people use a variety of signing styles from natural signing with minimal features of Chinese to a form of signing with more Chinese influences (see Figure 3.3). Having said that, I have never met a deaf person who would produce a form of signing that is at the Chinese end of the continuum, i.e., Signed Chinese. This is because Signed Chinese has never been introduced and used in deaf schools in Hong Kong. This is unlike 
the situation in Taiwan, for example, where Signed Chinese was invented and many character signs were created, so that a Chinese sentence can be signed character by character (Lee, Tsay, \& Myers, 2001). It may be possible that the teachers in deaf schools used a form of contact signing with more Chinese influence because of their limited knowledge of HKSL grammar and their first language interference. This happens commonly in hearing adult HKSL learners. Except for people who have deaf parents and who would be more likely to have acquired the language naturally, all other deaf people have to learn it from their peers and probably from their teachers (with limited signing skills) in a predominantly oral environment. This clearly would affect how one learnt a language and hence explain the diverse signing skills observed among signers.
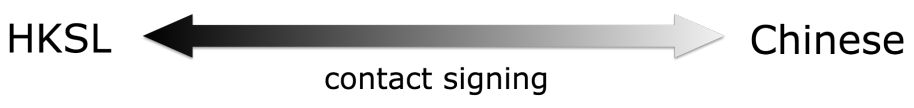

Figure 3.3: Contact signing continuum

Nevertheless, it is common to see a deaf signer switching his/her signing along the contact signing continuum to match the interlocutors. That is, one may use more mouthing when signing with a hearing person, but use a more natural way of signing when chatting with friends. It is worth noting that most of the informants who have deaf parents pointed out that they sign differently with their parents at home and with their classmates in school. Some said that they just assumed the language use in school and at home should be different and they did not realize the difference until they entered CSLDS. One told me that she sometimes has to interpret for her parents and friends, because her parents could not lip-read and her friends use a lot of mouthings. 


\subsection{Language contact in the deaf community}

Apart from Cantonese and written Chinese (traditional) which are used in Hong Kong, written English is also commonly used. Both Chinese and English are taught in schools, including deaf schools. Many people have English names including deaf people and fingerspelling may be used to introduce one's English name to another person. The one-handed fingerspelling system is adopted with modification in a few letters. Other than fingerspelling names, simple phrases like $\mathrm{O}-\mathrm{K}$, and the use of single letters e.g., when talking about a bus number or grades, fingerspelling is not frequently used in Hong Kong. Due to the influence of English, these alphabet signs may also be used to create new signs. One good example of this is the sign for email with the E-handshape moving from the sender to receiver (see Figure 3.4 below).

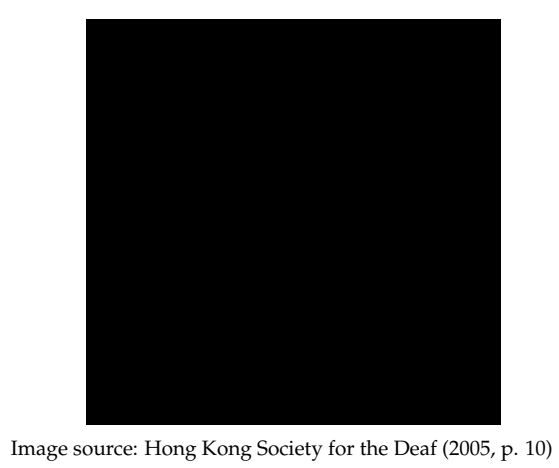

Figure 3.4: EMAIL

While it is not uncommon for signers in English speaking countries to fingerspell an English word to represent a concept that has no sign equivalent, Hong Kong signers resort to Chinese writing on palm or paper, tracing Chinese characters in the air (sometimes with the combination of character signs), or typing Chinese using their cell phone. The use of charac- 
Sociohistorical context for HKSL variation

ter signs and using signs to represent written characters is also common in sign languages having contact with written characters such as Taiwan Sign Language and Japanese Sign Language (Ann, 1998; Nonaka, Mesh, \& Sagara, 2015).

Loan translation is another strategy to represent a concept in Chinese literally through signs. For example, if a signer's name has the character 美 (means pretty), and she may create her name sign as PRETTY. This strategy is also used in creating new signs when new concepts arise, as new terms appear in Chinese and are made known to deaf signers for the first time. For instance, the term for internet access, 上網 (literally up net), is produced by a compound sign UP^NET. Yet, this is only one of the many ways to create new signs and this method can only apply to words where there are existing signs to represent the Chinese characters involved.

Even if there are signs to represent Chinese characters, this conversion strategy is not always straightforward and clear. One good example was found in the conversation data involving two older signers who went to the same oral school. Signer TKB talked about his experience in Melbourne. There is no sign for Melbourne in HKSL, the Chinese for Melbourne consists of three characters: 墨爾本 (a transliteration based on pronunciation) and the first character means 'ink.' TKB first signed the name sign of Melbourne used in Auslan. As his interlocutor, FBH, did not understand the sign, he made a sign imitating the action of grinding ink using an ink stick together with mouthing to refer to Melbourne. FBH got the meaning and briefly repeated TKB's sign, then he quickly signed BLACK. This indicated that FBH would sign the character 墨 as BLACK instead of the one used by TKB. This may due to two reasons. First, the most common colour 
of ink is black; second, the character for 'black' is 黑, which forms the upper part of the character 墨. Some researchers call this type of sign visual metonymic signs, i.e., they are based on a visual resemblance to Chinese characters (Fischer \& Gong, 2010). This way of creating signs may look complex and confusing to people who do not know HKSL and Chinese, but deaf people seem to manipulate these kinds of strategies quite well in order to communicate with each other using their entire repertoire of linguistic resources. It is similar to the situation described by Johnston (2002), where signers exploit Auslan to represent English in a useful and meaningful way. Additionally, even deaf children would have naturally made use of their linguistic resources to facilitate their own classroom learning, provided a sign bilingual school environment ${ }^{11}$ (Tang, Yiu, \& Lam, 2015). Nonetheless, making use of Chinese in creating new signs is just one of the many word-formation strategies in HKSL.

Another language contact situation is the contact with other sign languages like American Sign Language (ASL), CSL, and Taiwan Sign Language (TSL). Since it has been very difficult for signing deaf people to achieve tertiary education in Hong Kong, those who are capable typically would pursue further study in the United States. There are several deaf signers who have brought ASL back with them, after they finished their study. In addition, technological advances over the past years have made video sharing highly accessible. It is not surprising to see young signers learning and picking up ASL signs to fill the lexical gaps in HKSL through

\footnotetext{
${ }^{11}$ The sign bilingual school environment in Hong Kong is provided under the Jockey Club Sign Bilingualism and Co-enrolment in Deaf Education Programme: www.cuhk.edu.hk/cslds/jcslco/.
} 
watching online videos.

In addition, a surge of immigrants from mainland China since the handover in 1997 has introduced various CSL varieties to Hong Kong. This happens in the deaf school (as mentioned earlier in Section 3.3.5) and the deaf community as a whole. When a deaf pastor came back to do his missionary work after his study in a Taiwan seminary, a number of Taiwan signs were disseminated through churches. Indeed, the increasing social flow between Hong Kong, mainland China and Taiwan has promoted and encouraged borrowings among the three sign languages over the past decades.

To conclude, all the various sources of contact described above have contributed to lexical variation and phonological variation in HKSL to some degree.

\subsection{Chapter summary}

In this chapter, I have provided an overview of deaf education in Hong Kong from 1930s to 2000s. While both manual and oral schools co-existed for over twenty years before the mid-1970s, oralism predominated from the mid-1970s to 2000s. In this study, OMS included all private deaf schools and clubs for deaf children run by the Social Welfare Department. The majority of these schools used the Shanghai variety of CSL as the medium of instruction. Therefore, the modern form of HKSL is greatly influenced by this variety, and the two languages are known to belong to the same family. When OMS closed down in mid-1970s, many of their students were 
transferred to the oral schools and hence, spread the Shanghai variety to these schools.

During the oralism period, there were four deaf schools: HSD, VSD, CMS, and LSD. While all schools claimed to adopt the same language policy, there were discrepancies in practice. HSD was viewed as the strictest, VSD and CMS were considered as moderate, and LSD was the most open in allowing the use of signs together with speech. My interview data supported this observation. Most participants from HSD, VSD and CMS recalled some teachers using limited signs to teach and communicate with them. By contrast, most people from LSD remembered using cued speech during primary, and both speech and sign in secondary school. Those teachers who used signs were often the more experienced and had learnt signs from their students. By virtue of such learning, very few teachers would have reached a high proficiency level and most of them would be using a form of contact signing or sign supported speech. This in turn may affect the learning and signing of their students.

After finishing their study in deaf schools, deaf people of different schooling backgrounds gather and socialize with each other by going to deaf events, joining activities organized by deaf organizations or other social functions. Over the years, a modern form of HKSL has formed and a high degree of lexical variation has been observed. Nonetheless, the development of the language has been hindered by the prohibition of the use of sign language in education over 30 years. This has resulted in a considerable number of lexical gaps.

Most deaf signers are bilinguals in HKSL and Chinese, or even trilingual, as English is also taught in schools. Neologisms may use features 
other than HKSL. Signers would also exploit their linguistic resource of HKSL, Chinese, and English to create signs online to facilitate their conversation. Borrowing is another strategy available to Hong Kong signers. ASL, CSL, TSL and possibly other signed languages may all be the source of borrowing, given the condition for HKSL to contact with each of these languages. All of these language contact phenomenon have certainly added complexity to the picture of variation in the Hong Kong deaf community. 


\section{Chapter 4}

\section{Research methodology}

\subsection{Overview}

This chapter describes the research methodology of the current study. Section 4.2 outlines the research approach adopted in this study. Section 4.3 describes the characteristics of the participants and the recruitment process. Data collection and data coding are detailed in Section 4.4 and 4.5 respectively. Section 4.6 discusses how the data were analyzed and lastly, Section 4.7 explains the issue of multicollinearity and how it is mitigated.

\subsection{Research approach}

This research is a modified replication of the American Sign Language (ASL), Australian Sign Language (Auslan), and New Zealand Sign Language (NZSL) studies mentioned earlier (Lucas et al, 2001; McKee \& Mc- 
Kee, 2011; Schembri et al., 2009). As in these studies, two important principles underlie the approach to the current research. One is the principle of quantitative modeling and the other one is the principle of multiple causes (Young \& Bayley, 1996). The first principle addresses the quantitative approach needed for examining the forms of a sociolinguistic variable and their co-occurring linguistic environment and social phenomena. According to Tagliamonte (2006), "[t]he advantage of the quantitative approach lies in its ability to model the simultaneous, multi-dimensional factors impacting on speaker choices, to identify even subtle grammatical tendencies and regularities in the data, to assess their relative strength and significance" (p. 12). The second principle refers to the supposition that no one single contextual factor, linguistic or social, can adequately explain the variability in a given dataset. By coding as many conceivable constraints as possible, it is hoped that all potential factors can be captured in the model that best describes the variable phenomenon under study.

\subsection{Participants and recruitment}

By using non-random sampling and a snowball technique (Milroy \& Gordon, 2003; Schilling-Estes, 2007), fluent signers of different age groups from different deaf schools were recruited based on the following criteria:

1. born or became deaf before the age of 3

2. born or migrated to Hong Kong before the age of 3, and grew up in Hong Kong 
3. entered deaf school before the age of 7 (or 10 for older signers) ${ }^{1}$

Since the focus of this study is on sociolinguistic variation in HKSL, the above criteria are set to recruit both native and non-native HKSL users who learnt to sign either natively at home or started learning signs in local deaf schools when they were children. This aims to exclude HKSL users who were born hearing and were native speakers of Cantonese before being deafened, and those who had learnt another sign language, such as Chinese Sign Language (CSL), before they moved to Hong Kong and learnt HKSL.

Despite the preset criteria, three participants out of 68 participants recruited did not meet the criteria and their data were not included in the analysis. Among the three excluded participants, two migrated to Hong Kong after 3 years old, of whom one came at the age of 7 and the other at 9 . The third participant entered deaf school at the age of 13 and had studied for two to three years before moving to the United Kingdom with family when she was a teenager. One older participant entered deaf school at the age of 11 but was included because late admission was expected for people at her age and she had grown up in Hong Kong, using HKSL most of her life. Thus, the database under analysis consisted of the data from 65 participants. Due to time constraints, the conversation data of 40 participants were used for analyzing phonological variation, while data from all 65 participants was used for the analysis of lexical variation. A sub-sample of 40 was decided because school was a key factor group, and

\footnotetext{
${ }^{1}$ These age criteria followed the previous studies to ensure that HKSL was acquired early.
} 
participants were identified mainly with four schools. Thus, a sample of 40 allowed 10 participants in each subgroup to form a reasonable sample size for statistical analysis.

The basic demographic characteristics of the 65 participants (for lexical variation analysis) and the subset of 40 participants (for phonological variation analysis) are summarized in Table 4.1 and 4.2 respectively.

\begin{tabular}{|c|c|c|c|c|c|c|c|c|c|c|c|c|c|c|c|}
\hline \multirow[b]{2}{*}{ age } & & \multicolumn{2}{|c|}{ gender } & \multicolumn{5}{|c|}{ school } & \multicolumn{2}{|c|}{$\begin{array}{c}\text { language } \\
\text { background }\end{array}$} & \multicolumn{5}{|c|}{ region } \\
\hline & & F & M & OMS & HSD & LSD & CMS & VSD & HKSL & other & HKI & KLE & KLW & NTE & NTW \\
\hline $20-34$ & 23 & 12 & 11 & - & 11 & 4 & 8 & 9 & 5 & 18 & 2 & 5 & 2 & 6 & 8 \\
\hline $35-49$ & 21 & 13 & 8 & - & 8 & 8 & 5 & 5 & 1 & 20 & 4 & 3 & 3 & 5 & 6 \\
\hline \multirow[t]{2}{*}{$50+$} & 21 & 10 & 11 & 12 & 6 & 2 & 3 & 5 & 0 & 21 & 3 & 5 & 3 & 6 & 4 \\
\hline & 65 & 35 & 30 & 12 & 25 & 14 & 16 & 19 & 6 & 59 & 9 & 13 & 8 & 17 & 18 \\
\hline
\end{tabular}

Table 4.1: Demographic characteristics of 65 participants used for lexical variables

\begin{tabular}{|c|c|c|c|c|c|c|c|c|c|c|c|c|c|c|c|}
\hline \multirow[b]{2}{*}{ age } & & \multicolumn{2}{|c|}{ gender } & \multicolumn{5}{|c|}{ school } & \multicolumn{2}{|c|}{$\begin{array}{c}\text { language } \\
\text { background }\end{array}$} & \multicolumn{5}{|c|}{ region } \\
\hline & & F & $\mathbf{M}$ & OMS & HSD & LSD & CMS & VSD & HKSL & other & HKI & KLE & KLW & NTE & NTW \\
\hline $20-34$ & 12 & 7 & 5 & - & 4 & 3 & 5 & 5 & 3 & 9 & 1 & 2 & 2 & 1 & 6 \\
\hline $35-49$ & 12 & 5 & 7 & - & 3 & 5 & 4 & 3 & 1 & 11 & 3 & 1 & 1 & 2 & 5 \\
\hline \multirow[t]{2}{*}{$50+$} & 16 & 8 & 8 & 11 & 3 & 2 & 3 & 1 & 0 & 16 & 0 & 4 & 3 & 6 & 3 \\
\hline & 40 & 20 & 20 & 11 & 10 & 10 & 12 & 9 & 4 & 36 & 4 & 7 & 6 & 9 & 14 \\
\hline
\end{tabular}

Table 4.2: Demographic characteristics of 40 participants used for phonological variables

It is believed that the closing down of signing schools in the mid-1970s has had a great impact on the development of HKSL, and students of different oral schools have created distinct signs to accommodate their communication needs. As detailed in Chapter 3, before the mid-1970s, there were a few private manual schools, clubs for deaf children run by the Social Welfare Department, and the Hong Kong School for the Deaf (HSD). From the 1970s to 2000s, there were four oral deaf schools: HSD, Victoria School for the Deaf (VSD), Lutheran School for the Deaf (LSD), and Caritas 
Magdalene School (CMS). Considering the small number of students who studied in the private schools and in the clubs run by the Social Welfare Department, those participants who had been educated in these schools or clubs were grouped together and labeled as Old Manual Schools (OMS) in this project (see Section 3.3.1 on page 52). Hence, the sample included signers from five different schooling backgrounds: HSD, OMS, VSD, LSD, and CMS. As the OMS were all closed down around mid-1970s, the OMS group only had participants over the age of 50. Further, there were participants who had studied in more than one deaf school for two main reasons. First, following the closing down of OMS, some students were transferred to the newly opened schools. In my sample, three participants in their 50s had attended OMS, two were then transferred to CMS and one was transferred to HSD. Second, VSD only provided primary education. Those students who wanted to continue with secondary education were required to go to another deaf school. The majority of them went to CMS for the close link between the two schools stated in Chapter 3. In my sample, eleven participants went to CMS after VSD, while another seven participants entered HSD instead. There was one participant who went to three different deaf schools, but only the two schools he spent much time studying were coded. The one he had attended for only two years was excluded. By grouping participants according to the schools they had attended, I aimed to explore if certain variants were specific to certain schools.

Participants were divided into three different age groups: 18 to 34, 35 to 49,50 and above. These groupings produced a more balanced number of signers in each group. For the reason mentioned in the previous paragraph, the last age group consisted of participants from all different schools, whereas the first two age groups did not have signers with 
OMS background. Although there were no significant language policy changes in deaf education before the closing down of deaf schools in the 2000s, generational differences and language change were expected to be found by dividing the participants into younger, middle-aged, and older age groups.

Gender has been shown to play a significant role in sociolinguistic variation research in both signed and spoken languages (Cheshire, 2004; Leeson \& Grehan, 2004; Lucas et al., 2001; Schembri et al., 2009). Hence, both male and female signers were recruited. Like age and gender, region has been another main focus of variation studies. Regional variations have been found in most if not all the studies of national sign languages, as stated in Chapter 2. Some people in Hong Kong have claimed that HKSL has regional variations as well. In order to find out if regional variations in a regional language really exist in this small and highly dense city, participants living in various parts of Hong Kong were included. Depending on where they lived at the time of data collection, participants were grouped into five geographical areas: Hong Kong Island (HKI), Kowloon West (KLW), Kowloon East (KLE), New Territories West (NTW), and New Territories East (NTE) (see Figure 4.1). Over $85 \%$ of the participants had been living in the same area for ten years or more. There were six participants who had lived at their current address for less than seven years. In such cases, their previous address, which they had been living for a longer period than their current ones, or the area they grew up in, were used. This is because the place where one grew up or had been living at for a longer period would be more representative if regional variations do exist. 


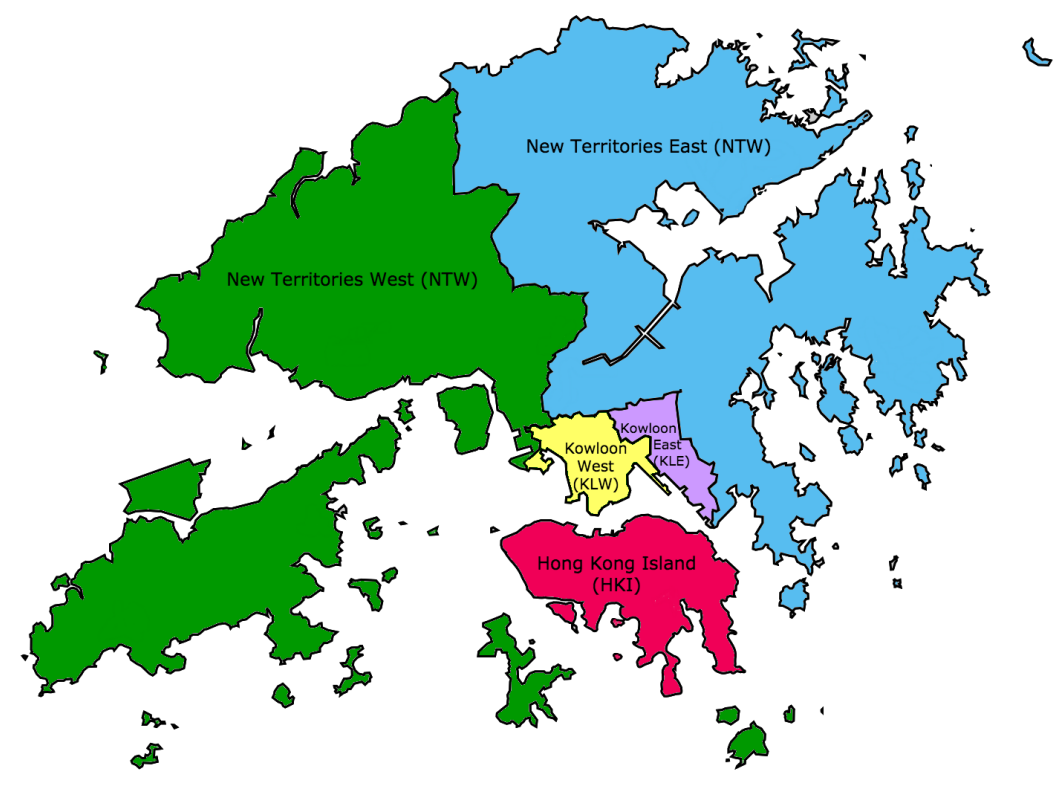

Figure 4.1: Hong Kong map showing the five regions

My sample also included signers with deaf parents, who acquired HKSL natively. They have a different language background from most of the deaf people who learnt HKSL later in schools. Since the studies of ASL and NZSL have shown that signers with different language backgrounds behave differently in their language use, it is important to include signers from both hearing and deaf families. In a study carried out in the United States, at least $92 \%$ of deaf children have hearing parents who do not know sign language (Mitchell \& Karchmer, 2004). I intended to recruit at least six signers with deaf parents in order to have a better sample size for statistical analysis. This represents $9.2 \%$ of my sample and reflects the projection of the general population, albeit the number may be too small for statistical analysis.

Social class is another social factor that has long been a focus of variation studies (Ash, 2013). The studies of ASL and Auslan included social 
class as a factor. However, as in the NZSL study, most Hong Kong deaf people have low socioeconomic status due to the poor education and limited employment opportunities. Therefore, social class is not considered in recruitment and the analysis. Nevertheless, the participants have different levels of educational attainment and they were coded accordingly to see if educational level would have any effect on the variable. Similarly, ethnicity is not taken into account for over $90 \%$ of the Hong Kong population are Chinese. The small number of non-Chinese deaf children, such as immigrants from South Asian countries, would go to the same deaf schools as their Chinese counterparts. No difference in the signing of non-Chinese deaf adults, who grew up in Hong Kong, is observed.

\subsection{Data collection}

Participants were recruited through the researcher's networks in the HKSL community, using a snowballing technique to extend to "friend of a friend." Since I am a hearing person and HKSL is not my first language, I invited two deaf friends, AL and AY, to work as research assistants on a voluntary basis. Both assistants were female, fluent HKSL signers and grew up in Hong Kong. Both research assistants helped recruit participants who met the criteria through their social networks. In addition, they assisted in the lexical elicitation task and in explaining the project to participants. Instructions were given to both assistants before commencing the data collection process. They were required to sign a confidentiality agreement form (Appendix A1) and were reimbursed for travel expenses. One of the research assistants had also participated in the study before she was recruited as a 
research assistant.

All the data collection sessions were done in dyads. When one potential participant agreed to take part after initial contact, he/she would then look for a partner based on the three criteria. In some cases, the research assistants arranged the dyad after seeking consent from both individuals who already know each other. All dyads were friends of each other, including one pair of brothers. Married couples were separated into different dyads, but there was one girlfriend-boyfriend pair. Many dyads were of similar age and schooling background. Since the participants were allowed to converse about any topic and the conversation data was used for phonological analysis, participant familiarity would not be expected to affect the data.

Some potential participants declined to participate for the reason that they would be videotaped, this was especially an issue when recruiting women over the age of 60 . As a result, there was only one woman aged over 60 in my sample. Another target group we found difficult to recruit was young males from LSD. This may be due to the fact that both research assistants and I have limited links with this group of deaf signers. Even when we had reached some potential participants, they either declined by saying "not interested in it," or failed to meet the second criteria - born or migrated to Hong Kong before the age of 3 . The first reason may relate to "a lack of awareness about the importance of this kind of research. This points to a lack of metalinguistic awareness and even to negative attitudes about ASL [HKSL in this case] and variation" (Lucas et al., 2001, p. 39). In fact, a few participants recalled having negative attitudes about HKSL when they were young or before working in the Centre for Sign Linguistics 
and Deaf Studies (CSLDS). The second reason reflected the fact that many deaf children who migrated to Hong Kong entered LSD, while many local deaf children were mainstreamed. As one of the young male participants from LSD told me, many of his classmates were from mainland China with different signing backgrounds.

In total, 34 dyads were filmed. I ran the entire session for eight of these dyads because no research assistants were available. For the other 26 dyads, one of the two assistants was there helping. All sessions were conducted within a single day except one dyad, which was filmed in two separate days. ${ }^{2}$ Some sessions were conducted in the home of one of the participants. Some sessions were conducted in research assistant AY's studio where she offered HKSL classes. For all other sessions, data were collected at the common areas in one of the universities. These areas are lounges for students, with sofa and coffee table, and there was space for setting up two camcorders. ${ }^{3}$

At the beginning of each session, an information sheet and consent form (Appendix A2 and A3) in Chinese were given to the participants. Either the research assistant or I would explain the forms in HKSL unless some participants told us they fully understood the Chinese description and they did not need translation in HKSL. After they signed a consent

\footnotetext{
${ }^{2}$ On the first day of filming, that dyad walked out of the camera's view in the middle of the filming, which made their conversation video quite short. I worried that there would not be enough data for analysis, so I requested them to film again on another day and they agreed.

${ }^{3}$ There may be people (mostly hearing students of that university) walking around during the filming, but the camcorders were not blocked.
} 
form, they provided basic information about themselves on a short demographic profile (Appendix A4).

Three types of data were collected in each session and all sessions were video-recorded. The first part was free conversation between the pair for approximately 45-60 minutes. In order to obtain natural data and minimize the effects of Observer's Paradox (Labov, 1972), participants were left alone after the camcorders were turned on. The second task was lexical elicitation which was done with individual participants separately. The research assistant showed the participants a set of 120 stimuli (pictures with or without Chinese characters equivalence in random order) to elicit signs for the concepts represented. This 120 lexical item list consisted of several semantic domains, including colour, number, kinship, country/region, food, local place, festival. All items were observed to have variants based on my own experience and by comparing HKSL books and the dictionary. ${ }^{4}$ The lexical elicitation task was done by using either a laptop (for the first few sessions) or an iPad, showing slides of stimuli as in Figure 4.2 below. After the first few sessions, some participants failed to recognize the concepts represented by a few pictures, mainly for the country/region domain. ${ }^{5}$ Consequently, a few tokens were missed. A couple of stimuli were then revised by adding Chinese words, changing pictures or both. The revised set was then used throughout the remaining sessions. The third task was a short interview. I interviewed all the participants

\footnotetext{
${ }^{4}$ See www.uclan.ac.uk/research/explore/projects/sign_language_typology.php for more on semantic domains in other sign languages.

${ }^{5}$ Before I started to collect the data, I had tested the lexical elicitation part with one of my deaf friends, who did not participate in this study. This signer could recognize all the concepts shown in the slides and gave me the signs for each concept.
} 
individually about their background, social network, and language use, based on a list of guiding questions (Appendix A5). It has been shown in sign language research that deaf signers are very sensitive to the hearing status of their interlocutors (Lucas \& Valli, 1992). That is to say, participants may sign differently to me, a hearing person, than to my deaf research assistant. To ensure consistency and to have a better understanding of all the informants, I conducted all the interviews myself and the interview data was only used in the analysis of the first phonological variable - DEAF/HEARING due to the small number of tokens found in the conversation data (see Section 4.5.2.1 on page 97 for details).

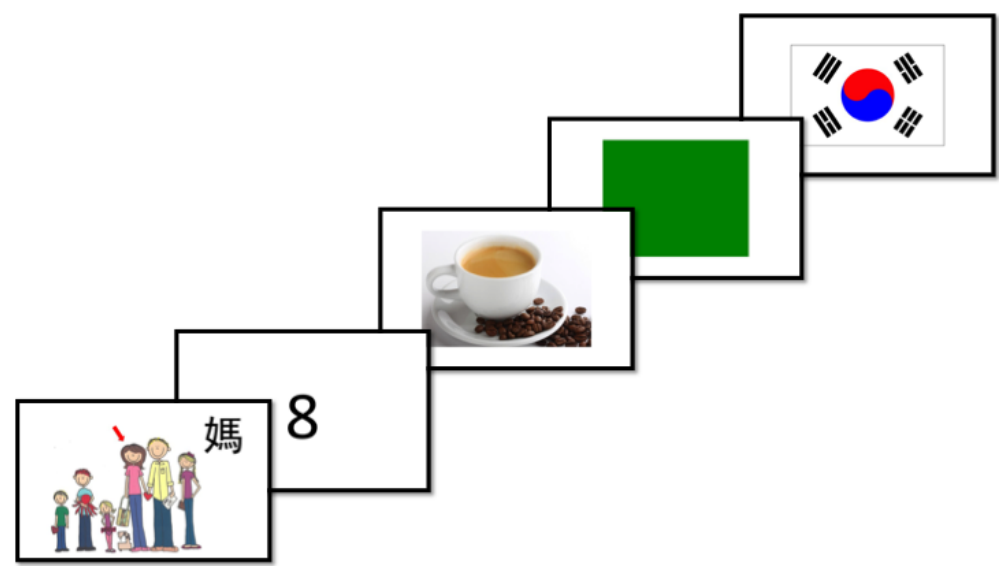

Figure 4.2: Example of stimuli shown to participants

The second and third tasks were conducted at the same time when a research assistant was present. In other words, while the research assistant was eliciting signs from one participant, I was interviewing the other participant. The participants swapped after finished one task. In cases where there was no research assistant, I did the elicitation task myself and one of the participants had to wait until I finished both tasks with their conversation partner. Since the elicitation task focused on lexical items, 
the influence of the hearing status of the elicitor was believed to be minimal. In most situations, the elicitation task and interview were conducted individually to avoid possible influence from the other signer. In a few cases, the elicitation task and interview were conducted in the presence of the conversation partner. The interviewees acknowledged the presence of their conversation partner and the latter agreed not to interrupt during the whole process. After finishing all three tasks, participants were remunerated for their time.

\subsection{Data transcription and coding}

All data were coded using ELAN (EUDICO Linguistic Annotator), professional software for the creation of complex annotations on multimedia resources. Its ongoing advancement by the developer, Max Planck Institute for Psycholinguistics in Nijmegen, in collaboration with sign language researchers has enhanced the functionality and usability for sign language research, including sign language corpora (Crasborn \& Sloetjes, 2008, 2014).

In total, there were around 8 hours of lexical data from 65 participants, and roughly 13 and 31 hours of interview and conversation videos from 40 participants respectively. Only selected and relevant signs, plus related features were annotated for analysis. The following sections detail how different parts of the data were coded. 


\subsubsection{Lexical data}

For the lexical data, 52 out of the 120 elicited concepts were transcribed and 51 of these were coded because no variation was found for the colour sign YelLOW. ${ }^{6}$ Hence, the expected number of tokens would be 3,315 (51x65). However, there were 24 missing or erroneous tokens due to several reasons. As mentioned in Section 4.4, some participants could not identify the target concept before the elicitation process was revised. Some participants responded they "do not know the sign" or "forgot the sign" for a certain concept; this usually happened with country/region signs. A few participants gave the same signs for two concepts by mouthing the Cantonese word for the less common concept, such as GREY for SILVER with mouthing 銀 /ngan ${ }^{4}$ / (Cantonese for silver). One informant gave the sign for $\$ 3$ when she prompted by the stimulus 3 .

On the other hand, 42 out of 65 participants gave more than one variants for at least one concept. This added 109 more tokens for coding. As a result, the total number of tokens coded was 3,315-24+109 $=3,400$. The number of tokens of each concept is tabled in Appendix B. Unlike the studies of ASL, Auslan, and NZSL, which selected the first variant or asked for preference when two or more variants were given, I included all variants given by each informant. The reason was that several participants explicitly expressed that there are two signs for a certain concept when prompted. When asked which variant they prefer, they would either say "no preference" or "depending on who they are talking to." This showed

\footnotetext{
${ }^{6}$ Due to time contraint, 52 concepts were selected and they mainly belong to the semantic fields that have been studied in other signed languages.
} 
that Hong Kong signers are aware of the lexical variations in their language and they use more than one variant for certain concepts in their everyday life. In this situation, it would be inappropriate to choose just one variant for analysis. ${ }^{7}$

Among the 51 concepts under examined, twelve were colours, seven were kinship terms, eighteen were numbers, eight were countries/regions, two were the signs DEAF and HEARING (the first target phonological variables in this study). The remaining four were possible homonyms of four colours, which means the same form can refer to either concept in each pair: BROWN vs COFFEE, ORANGE vs ORANGE (fruit), PURPLE vs GRAPE, RED vs HUNG-HOM (local place). These four pairs were further reviewed to check if they were produced identically by the participants. They were then coded accordingly to see if any social factors would associate with the pattern of using homonyms.

In most cases, variants that differed in only one phonological parameter were considered to be phonological variants, as in previous studies (Lucas et al., 2001; McKee \& McKee, 2011). They were coded by adding letters to the sign label, such as BLUE1a and BLUE1b in Figure 4.3. Separate lexical variants with distinct phonological representation were coded using numbers, like BROWN3 and BROWN4 in Figure 4.4.

\footnotetext{
${ }^{7}$ Note that by not forcing signers to choose one preferred form over others, this study constitutes a more complete picture of the variation in HKSL than previous studies of lexical variation in sign languages may have.
} 


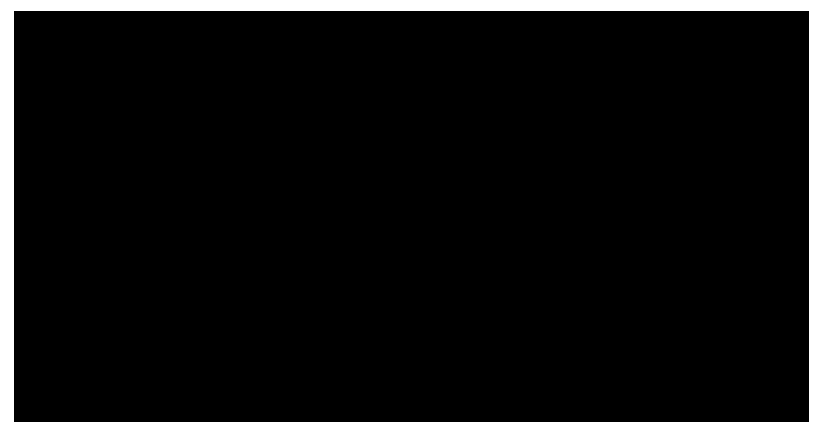

Figure 4.3: Phonological variants of BLUE

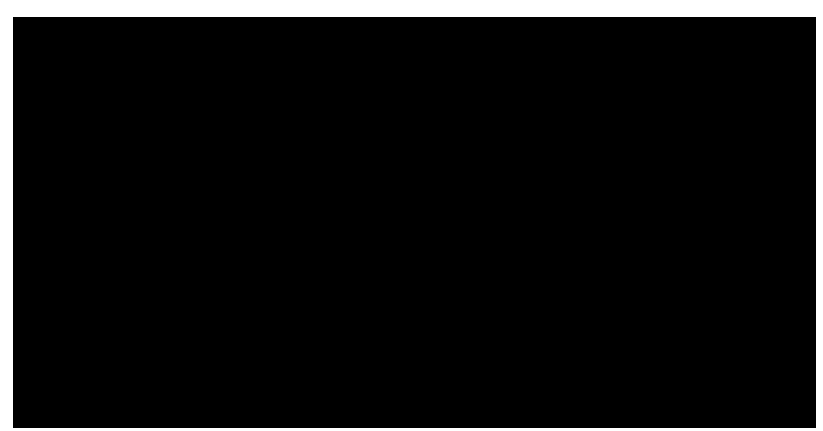

Figure 4.4: Separate lexical variants of BROWN

However, as Lucas et al. (2001) cautioned, "separating out what is truly a separate variant from what is a phonological variant of another variant is not always completely straightforward" (p. 180). There exist several lexemes that have variants which differed in more than one parameter, yet they look phonologically related to each other. One example is the five variants of GREEN illustrated in Figure 4.5. GREENa, GREENb, and GREENC differ in palm orientation only, with palm out, palm in, and palm down respectively, while wiggling the extended index and middle fingers (Uhandshape). Though GREENc also involves bending of the two fingers, it looks more like a phonological variant of GREENa and GREENb rather than a separate lexical variant. When comparing GREENC and GREENd, the only difference is the direction of the two extended fingers. While the 
former points out, the latter points to the side. The difference between GREENd and GREENe is location, as the former is articulated in front of the face. All five variants are consistent in their movement and handshape, they vary in their palm orientation and location in one. It is arguable to treat GREENC as a distinct variant from GREENa and GREENb, and to see GREENd and GREENe as subvariants of GREENc. Nonetheless, they appear to evolve from the same origin, which is probably GREENb, imitation of grass to signal green (further explained in Chapter 5). In such case, it is more convincing to regard them as five phonological variants.

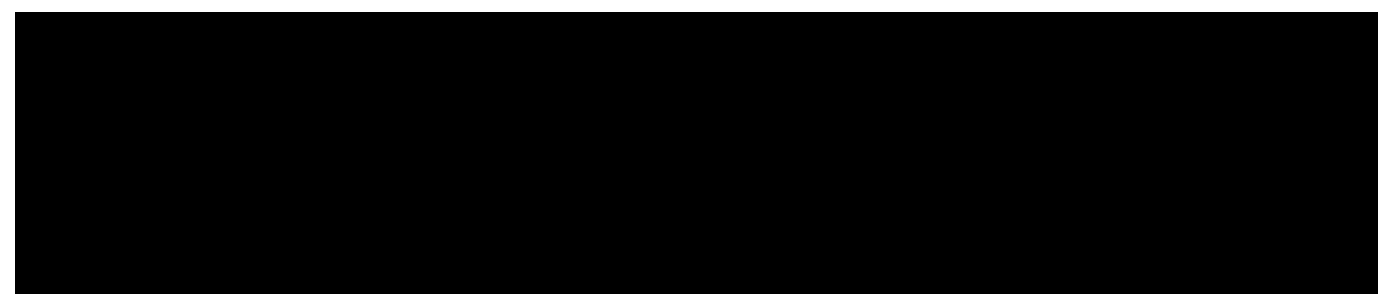

Figure 4.5: Phonological variants of GREEN

Another issue in classifying variants was the judgement and grouping of compounds. Since the participants could produce more than one sign for each stimulus item, sometimes it was difficult to judge whether the signer was producing a compound or two separate variants. Although there were cues such as reduction and deletion of movement segment (Liddell \& Johnson, 1986; Sutton-Spence \& Woll, 1999), many decisions were not easy to make. After deciding a response was a compound rather than two distinct variants, the next problem was to decide how to group the compound. There were compounds made up of the same signs but produced in a reverse order. For instance, one signer would produce RED^GRAIN for pink, while another signer would make it GRAIN`RED. In this case, I grouped them as a variant with a sublexical variant. In cases 
where only one part of the compound was the same, they were treated as two lexical variants.

\subsubsection{Compound signs}

After a preliminary investigation of colour signs, it seemed that older signers are more likely to use compound signs than younger signers. That is, they used two signs together to refer to a single concept. For example, an older signer may produce RED^LITTLE to mean pink as in Figure 4.6, while a younger signer may produce a simplex form PINK1a as in Figure 4.7. In order to further examine this phenomenon, I also coded for simplex and compound wherever applicable. Twenty-one out of the 51 concepts under examination appeared to be produced with a compound form by at least two informants. Two concepts (MOTHER and RELATIVES) for which only one signer produced a compound form were excluded, because those two lexical items do not normally have a compound variant, i.e., these compounds seem to be idiosyncratic innovations. Only simplex forms were given for the remaining 28 concepts. If a signer produced more than one variant and included a compound form, it would be coded as 'compound.' No signer produced two or more compound forms for one single concept. There were 20 missing or erroneous tokens in this subset. A total of 1,345 tokens were coded. These 21 concepts consisted of all semantic domains under study except for numbers. They are listed in Appendix C. 


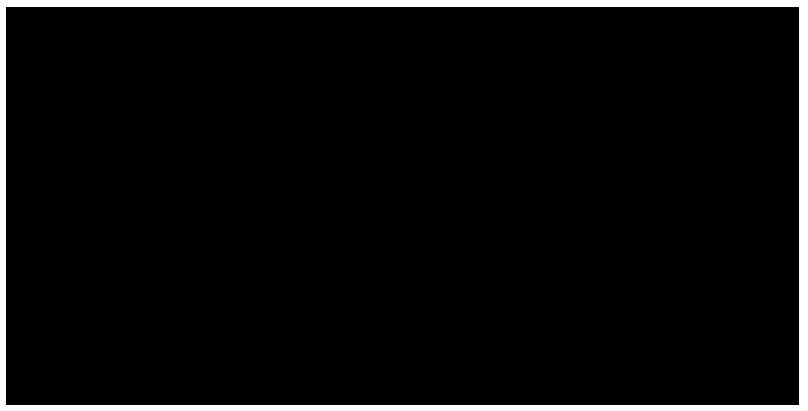

Figure 4.6: Compound variant of PINK

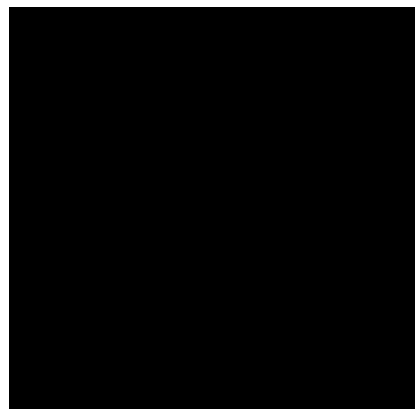

Figure 4.7: Simplex variant of PINK

\subsubsection{2 'Citation form'}

As described in Chapter 3, HKSL was not used formally in deaf schools from the mid-1970s until mid-2000s. Organizations offering HKSL classes have developed their own teaching materials. It is not surprising to see different signs for the same concept appear in different sign language books. The only dictionary of HKSL has yet to include all sign variants. Hence, it would be inappropriate, if not impossible, to call one of the sign variants the citation form. In spite of this, it is true that some variants occur more frequently and are more popular than others. With a view to explore which variants are more common in general, I juxtaposed sign pictures of the concepts under examination from five HKSL publications, includ- 
ing the dictionary (Appendix D). Three numbers: TWELVE, TWENTY-ONE, TWENTY-SEVEN, were not found in all publications. Though pictures of 47 lexemes were juxtaposed for comparison, some lexemes appeared only in one, two, three or four publications, but not all. Consequently, I chose 20 lexemes that appeared to have the same form in three or more of the five publications and considered that form to be the 'citation form' for this sub-study. ${ }^{8}$ For example, the sign MOTHER in Figure 4.8 appears to be the same in all publications; while only two publications show the same sign for ITALY as in Figure 4.9. Hence, MOTHER is included in the 'citation form' list, but ITALY is not. Based on this definition of 'citation form,' the 20 concepts identified were further coded as cf (citation form) or -cf (noncitation form). There were two missing or erroneous tokens in this subset. A total of 1,298 tokens were coded for analysis.

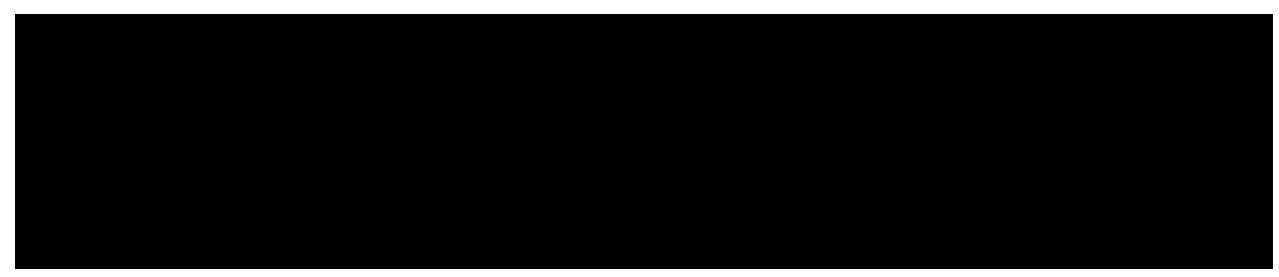
(a) 1972
(b) 1985
(c) 2004
(d) 2005
(e) 2007

Figure 4.8: MOTHER in different HKSL publications

\footnotetext{
${ }^{8}$ Some marginal cases were not treated as 'citation form.' For example, the sign BLACK has mainly two forms. Two books showed one and another two books showed the other one, the dictionary displayed both variants (See Appendix D, Figure 1).
} 


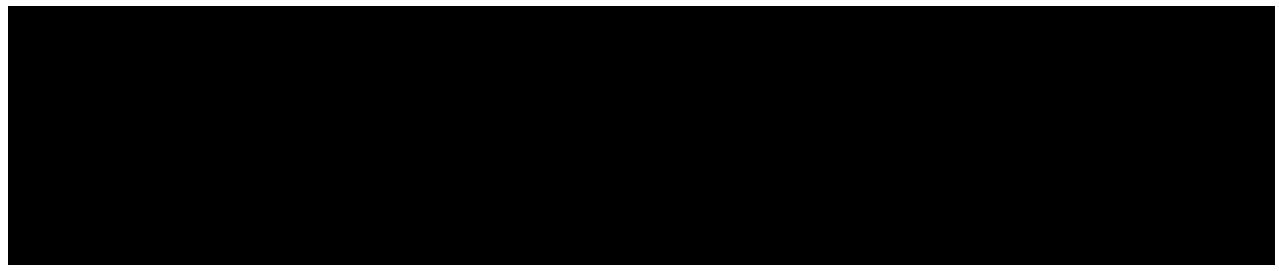

(a) 1972

(b) 1985

(c) 2004

(d) 2005

(e) 2007

Figure 4.9: ITALY in different HKSL publications

\subsubsection{Number signs: one-handed versus two-handed}

In HKSL, numbers above ten can be produced with either one or two hands. Figure 4.10 illustrates the one-handed and two-handed variants of the sign for thirteen. A shift from two-handed to one-handed signs has been observed in other signed languages (Frishberg, 1975; Stamp et al., 2015; Woll, 1987). This shift has also been found to correlate with some social factors as delineated in Chapter 2. Thus, numbers above ten were further coded as one-handed or two-handed. If a signer produced both one-handed and two-handed variants for a certain number, it would be coded as 'both.' Among the selected number signs, there was a total of 15 number signs coded and they were numbers ELEVEN, TWELVE, THIRTEEN, FIFTEEN, SEVENTEEN, EIGHTEEN, TWENTY, TWENTY-ONE, TWENTY-TWO, TWENTY-THREE, TWENTY-FIVE, TWENTY-SEVEN, TWENTY-EIGHT, THIRTY, and HUNDRED. These 15 numbers were further recoded into four different classes: tens, twenties, twenty/thirty, and hundred, in order to investigate what kinds of numbers are more likely to be signed with two hands. This categorization system differentiates (i) tens and twenties (made by a sequence of two handshapes, for example, TWELVE consists of a 1-handshape and a 2-handshape; TWENTY-FIVE consists of a 2-handshape and a 5-handshape; (ii) twenty/thirty (the major variant of each is formed by closing the se- 
lected fingers to make a fist); (iii) hundred (which has basically three compositionally different variants). It was decided to code tens and twenties separately because even though they are compositionally similar, there seemed to be a difference in how often one or two-handed signs were used. There were no missing or erroneous tokens in this subset. A total of 975 tokens were coded for analysis.

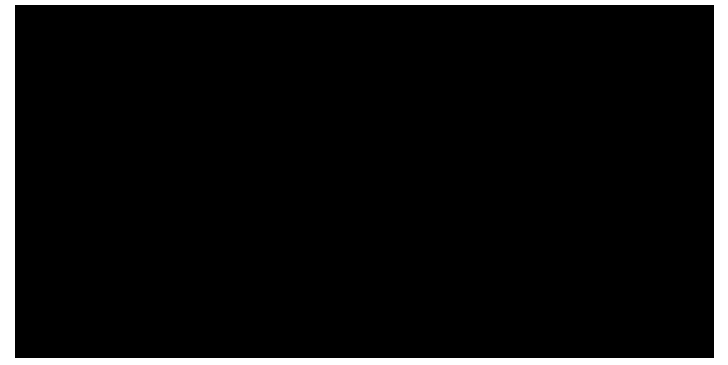

(a) One-handed form

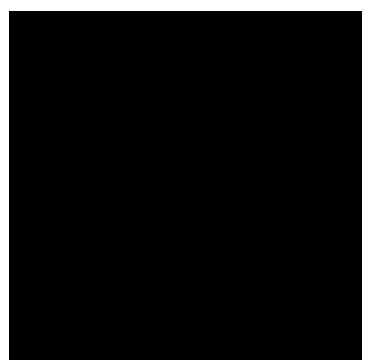

(b) Two-handed form

Figure 4.10: Variants of THIRTEEN

\subsubsection{Conversation data}

The conversation data from 40 of the 65 participants were coded for studying two phonological variations in this study. The first one is the DEAF/ HEARING variables, which are known of having at least two forms for each, and DEAF and HEARING are minimal pairs differing in handshape only. The second one is the location variable in the class of signs exemplified by KNOW, GOVERNMENT, and CRAZY (see Figure 4.11), which are canonically produced at the forehead or temple. The following two sections detail how the phonological variables were coded. 


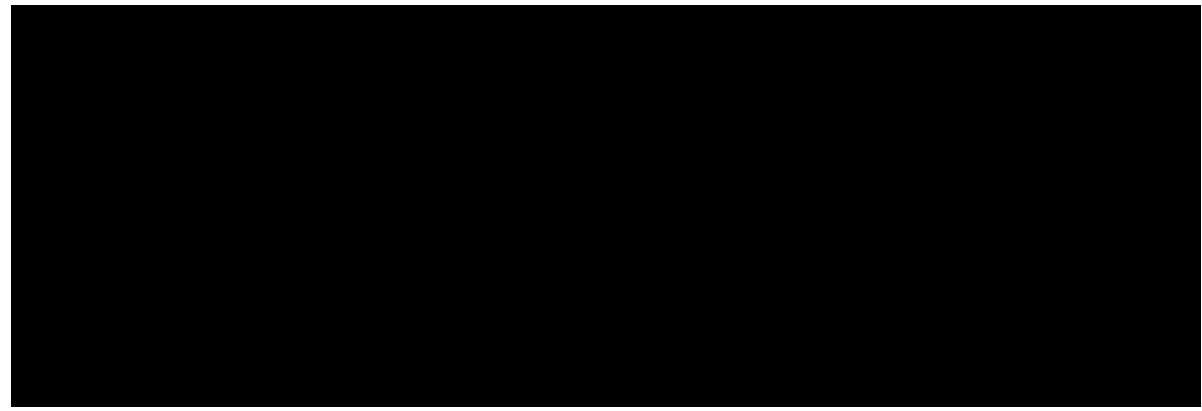

Figure 4.11: Examples of target signs: KNOW, GOVERNMENT and CRAZY

\subsubsection{Variation in the signs DEAF and HEARING}

One of the main reasons for choosing these two signs for examination is that DEAF is directly linked with the deaf identity and HEARING is used to refer to all non-deaf people. Both signs are very common in deaf discourse. Exploring variation in these two signs will not only help us understand HKSL and the signing community, but will also shed some light on the relationship between language and identity in general.

The sign DEAF was also investigated in the ASL study, in which three different forms were examined. In fact, the citation form of ASL DEAF is similar to one of the HKSL DEAF variants, just different in handshape. While the 1-handshape (index finger) is used in the ASL sign, the BADhandshape (pinky finger) is used in the HKSL sign. Both signs are produced by moving the selected finger from ear to chin. Figure 4.12 illustrates the two signs. Following the ASL study, the "Hold-Movement" model proposed by Liddell and Johnson (1989) is adopted. This model views a sign as composed of sequences of movement and hold segments, each segment containing a bundle of features including handshape, location, orientation, and nonmanual signals (Liddell \& Johnson, 1989). As 
Lucas et al. (2001) explained, "as Liddell (1993) amply demonstrates, it allows not only for an accurate account of the description of any individual sign but also for an accurate account of phonological processes such as assimilation, metathesis, epenthesis, and segment deletion, processes that play central roles in variation" (p. 20).

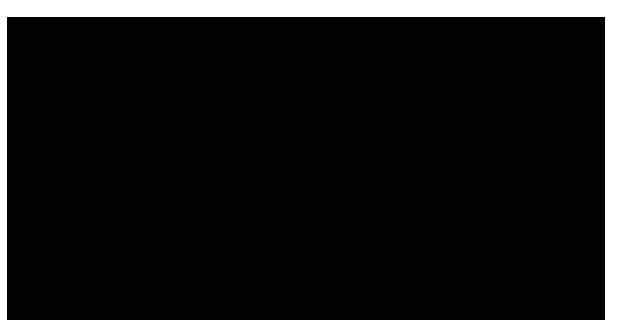

Image courtesy of Dr. Bill Vicars \& www.lifeprint.com

(a) ASL

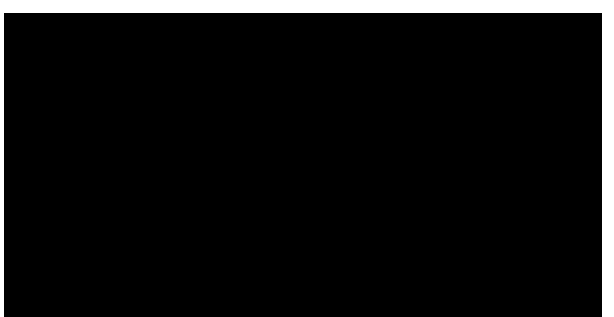

(b) HKSL

Figure 4.12: DEAF in ASL and HKSL

The target signs in the current study include both DEAF and HEARING. As explained earlier, the two signs are a minimal pair in HKSL with only one parameter difference, which is the handshape. The sign HEARING is composed of a GOOD-handshape (thumb) as opposed to the BADhandshape (pinky finger) in the sign DEAF. It should be noted that the variant of DEAF as shown in Figure 4.12b was originally a compound of DEAF and MUTE. It is evident in the first sign language publication, two separate entries for DEAF and MUTE were recorded in the book, as shown in Figure 4.13 (Goodstadt, 1972, p. 229). Since it was very hard (if not impossible) for deaf people to acquire speech in the old days, most prelingually deaf people could not speak and were called deaf-mute. Deaf people would also combine the two signs DEAF and MUTE when talking about themselves. This lexicalized compound was then viewed as one single sign in describing or referring to someone who is deaf. Though it is not 
sure whether the compound was formed as DEAF^MUTE or MUTE^DEAF when it was used at the very beginning, it seems that the first one (moving the pinky finger from ear to chin) became the 'citation form.' Figure 4.14 demonstrates the same sign printed in two HKSL books published by the same deaf organization at two different times (Hong Kong Society for the Deaf, 1985, Book 2, p. 10; Hong Kong Society for the Deaf, 2005, p. 3). Note that both the Chinese and English terms have changed over time, which reflects the societal view on deaf people at different points in time.

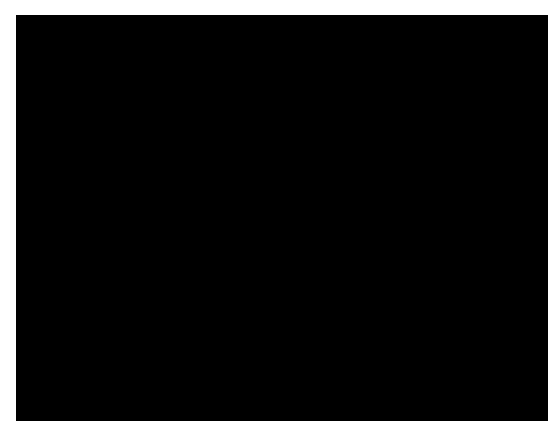

Figure 4.13: DEAF and MUTE in the first sign language book (1972)

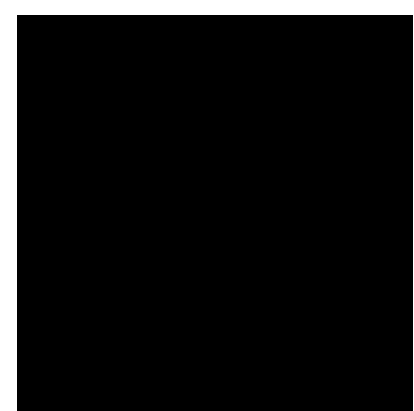

(a) $1980 \mathrm{~s}$

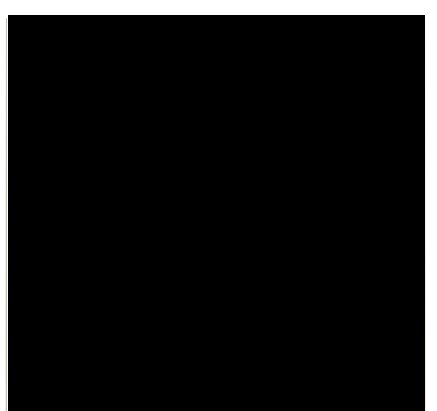

(b) $2000 \mathrm{~s}$

Figure 4.14: DEAF in HKSL books published in different years

On the other hand, the sign HEARING shows another pattern of change when we compare publications at different times. In the first book, the sign begins below the mouth and moves up to the ear (see Figure 4.15, Good- 
stadt, 1972, p. 229). In all the books published later (from 1980s to 2000s), the sign begins at the ear and ends near the corner of the mouth (see Figure 4.16, Hong Kong Society for the Deaf, 1985, Book 2, p. 10; Hong Kong Society for the Deaf, 2005, p. 4). The later version indicates the process of metathesis. Although the chin-to-ear version of HEARING appears to be the earliest form documented, no chin-to-ear version of DEAF has been found in any HKSL publications. Interestingly, despite this difference observed in the print sources, each of the signs has five variants parallel with each other in the current data (detailed in the coming paragraphs).

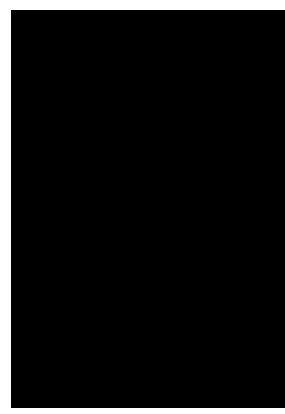

Figure 4.15: HEARING in the first sign language book (1972)

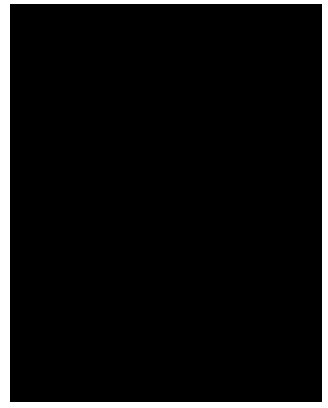

(a) $1980 \mathrm{~s}$

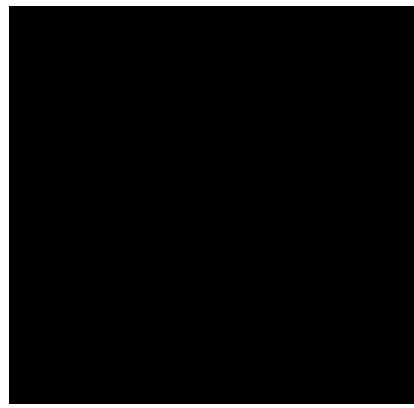

(b) $2000 \mathrm{~s}$

Figure 4.16: HEARING in HKSL books published in different years

Based on the "Hold-Movement" model, the ear-to-chin or the chin-toear variants of both DEAF and HEARING consist of a hold, a movement, 
and a hold (HMH). Unlike DEAF in the ASL study where the variation in location was modelled, ${ }^{9}$ the variation in movement was far more prominent in the HKSL signs DEAF and HEARING. Therefore, the variation in movement pattern in both DEAF and HEARING is the primary attention in the analysis described later. Having said that, the location variation was indeed noticed and coded. ${ }^{10}$

The conversation data for coding was the same as the one used for location variation analysis, except for one participant. ${ }^{11}$ The whole conversation of each of the 40 participants was viewed and coded for both DEAF and HEARING. In situations where the target sign was not clear due to the signer's posture, the viewing angle, or some other issue, those signs were ignored. ${ }^{12}$ Besides unclear signs, which made it difficult or impossible to code, 'impeded signing' was not coded as well. In such situation, the signer's elbows were resting on his/her own legs or chair armrest, and the articulation of the target sign may be affected in some way.

A few tokens of MUTE (pinky finger touched the chin/the corner of

\footnotetext{
${ }^{9}$ It can be argued that there is variation in movement among the three variants in the ASL DEAF. The ear-to-chin variant moves downwards, the chin-to-ear variant moves upwards, and the contact-cheek variant just contacts and holds without movement. Nonetheless, the researchers focused on the variation in location for analysis.

${ }^{10}$ The codings for location here were ear, cheek, chin, ear-to-chin, and chin-to-ear.

${ }^{11}$ One participant of the 40 selected participants (they were chosen when location variable was first coded) produced just a few tokens of DEAF and did not produce any tokens of HEARING. However, her interlocutor produced a fair amount of both signs. Therefore, data from her interlocutor (who was not coded elsewhere) replaced her conversation data when coding for DEAF and HEARING variables.
}

${ }^{12}$ There were two cameras setup for filming, one for each signer. 
the mouth) were identified and they were not coded as they clearly signified cannot speak in the context. Tokens with the same form but which convey the meaning of deaf in the context were coded as DEAF. ${ }^{13}$ Nevertheless, three participants did not produce either target sign throughout the whole conversation, seven participants produced none or just one token of HEARING, and one participant produced only one unclear token of DEAF. Hence, for these 11 participants, there were not enough tokens for statistical analysis. In order to obtain more tokens of both target signs, the interview data of these 11 participants was also coded. Since the interview topic was about their deaf experience, it was expected that the target signs would occur frequently in the interview data and this was the case for most people. Consequently, a total of 945 tokens of both DEAF and HEARING were coded, in which 645 tokens were coded from the conversation data, and 300 were coded from the interview data. The five phonological variants of both DEAF and HEARING coded are described below (note that the selected finger refers to pinky finger in DEAF and thumb in HEARING):

1. HMH: the selected finger begins at the ear and moves to the chin or vice versa, holds briefly at the beginning and the end

2. MMM: the selected finger moves back and forth along the line between the ear and the chin without hold at any point, the elbow joint is involved to form a path movement (sometimes the wrist joint is involved too)

\footnotetext{
${ }^{13}$ Following the practice of ID-glosses (Johnston, 2010), all the tokens of MUTE were annotated as MUTE on the gloss tiers. The tokens meaning deaf in the context were annotated as DEAF on the D/Htarget tiers, i.e., the tiers for the variables DEAF and HEARING.
} 
3. $\mathrm{MH}$ : the selected finger moves from the ear to the chin or vice versa, then holds briefly at the end

4. M: the selected finger moves back and forth along the line between the ear and the chin without hold at any point, only the wrist joint is involved to form a local movement

5. H: the selected finger contacts the ear, cheek or chin and holds briefly

Since movement is varied, it is logical to think that the phonological environment related to movement may have effects on signers' choices among these five variants. The movement of the preceding and following sign were coded. Basically, movements can be classified into two types: simple and complex. Simple movement consists of a single local movement or a path movement, where local movement is associated with the wrist, knuckle, or finger joints and path movement involves the shoulder or elbow joints. Movements are complex if more than one type of movement are involved (Tang, 2007). Besides movement, I also coded for other phonological environments similar to the location variable, i.e., the change in hand dominance, whether the preceding/following segment is a sign or a pause, the location of the preceding/following segment, and contact of the preceding/following segment. Since it is still unknown how the bundle of features may affect each other in phonological processes, I included the location of the surrounding phonological environment. Additionally, as previously stated, the location of the target signs DEAF and HEARING did appear to vary and this was coded. Some variants may favour a certain location. If the location of the surrounding phonological environment turned out to be a significant constraint, I could reexamine whether it is primarily conditioning the movement or the location of the target signs. 
Apart from the phonological environment, the grammatical class of the target signs DEAF and HEARING was also coded, based on the coding scheme of the ASL study. Because interview data was used, the genre was also coded. All the linguistic factors coded are as follows:

1. Grammatical function: noun (e.g., DEAF KNOW 'deaf people know'), adjective (e.g., MOTHER FATHER DEAF GIVE-BIRTH-TO ... 'Deaf parents gave birth to ...'), predicate adjective (e.g., PRO.3 DEAF '(s)he is deaf'), compound (e.g., DEAF^SCHOOL 'deaf school')

2. Change in hand dominance: no (no change in hand dominance before and after the target sign), before (change in hand dominance between the target sign and the sign before ), after (change in hand dominance between the target sign and the sign after), both (change in hand dominance between the target sign and both the sign before and after)

3. Preceding segment: sign, pause (target is the first sign) ${ }^{14}$

4. Movement of preceding segment: no (preceding sign consists of a hold segment only/preceding segment is a pause), simple (involved a single local or path movement), complex (involved more than one type of movement)

5. Location of preceding segment: high (at ear or above), middle (between ear and chin), low (chin or below/preceding segment is a

\footnotetext{
${ }^{14}$ The small number of meaningful gestures was coded as sign in preceding and following segments.
} 
pause $)^{15}$

6. Contact of preceding segment: no (no contact/preceding segment is a pause), head, hand, body ${ }^{16}$

7. Following segment: sign, pause (target is the last sign)

8. Movement of following segment: same codes as for movement of preceding segment

9. Location of following segment: same codes as for location of preceding segment

10. Contact of following segment: same codes as for contact of preceding segment

11. Genre: conversation, interview

Regarding social factors, the coding scheme is the same as the one adopted for location variable and is outlined in Section 4.5.3 about interview data.

\footnotetext{
${ }^{15}$ Unlike the coding for the location variable where 'not applicable' (NA) is coded if a change in hand dominance was involved, the preceding location of the hand used for the target sign was coded, i.e., where the hand was before the hand produced the target sign. This is to avoid NA codes that have to be removed before fitting the model in Rbrul analysis.

${ }^{16}$ Again, to avoid NA codes, the preceding contact of the hand used for the target sign was coded, i.e., which part of the body did the hand touch before the hand produced the target sign.
} 


\subsubsection{Variation in location}

The target signs were lexical items articulated at or above the forehead, either in touch with or close to the forehead area in their citation form. ${ }^{17}$ These signs have been observed to vary in location lower than the forehead in everyday conversation. Following the Auslan and NZSL studies, only signs made at the forehead region in their citation form were included and lexicalized compound signs having the second segment below the eyebrow ridge were excluded (Schembri et al., 2009). Likewise, two groups of signs were also excluded in order to get a more homogeneous set of data. The first group was signs involving two locations: the first at the forehead region and the next lower than the forehead region such as GAY (see Figure 4.17). The second group was target signs with negative incorporation, in which a bound, twisting outward movement is incorporated in a verb sign to indicate it is negated, for example THINK-neg (see Figure 4.18) because this bound movement may drop below the forehead area.

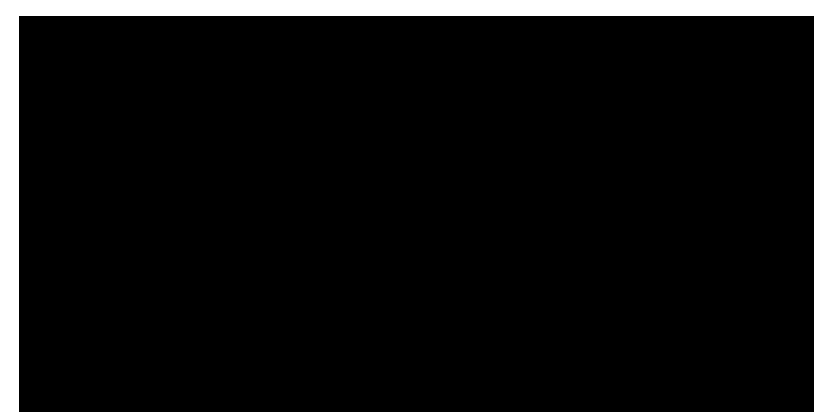

Figure 4.17: GAY

\footnotetext{
${ }^{17}$ Citation form here means the form that is illustrated in the dictionary and sign language books, and is taught in sign language classes. The use of the term citation form is different from the earlier one in analyzing lexical variation. Therefore, the quoted form 'citation form' is used in Section 4.5.1.2.
} 


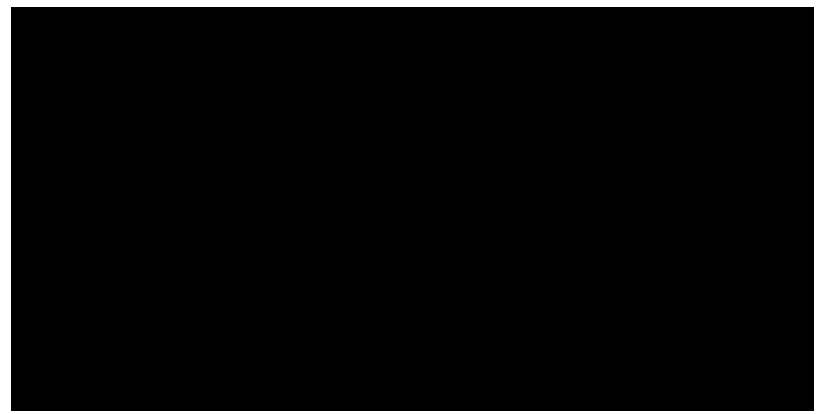

Figure 4.18: THINK-neg

As discussed in Section 4.5.2.1, unclear signs and 'impeded signing' were not coded. When the signer's elbows were resting on his/her own legs or chair armrest (i.e., impeded signing), and the target sign was obviously pulled down as a non-citation form due to the lean. In one instance, the signer deliberately tilted her head to articulate the signs at forehead position, i.e., the location in citation form, and the target signs were included and coded as citation form. In other cases, coding resumed once the signer's elbows were not resting on anything. Since the phonological environment was also coded for possible effects of linguistic factors, a target sign was not coded if its preceding or following hand action was not a sign, such as pushing up some sliding down spectacles.

Coding started from the beginning of the conversation until 20 target signs were sampled from each of the 40 participants, resulting in a total of 800 tokens (see Table 4.3 on page 110 for a complete listing of all target signs and their frequency in the database). In some studies, taking only the first 20 tokens from a participant might return a conservative measure of the variation, however, in the case of HKSL, this is arguably less of a problem than in more standardised languages where there are recognised 'formal' and 'casual' indexes for certain variants. Target signs produced on 
or above the eyebrow ridge were coded as citation form (cf), while those produced below the eyebrow ridge were coded as non-citation form (-cf). For double-handed target signs, in which both hands have the same handshape and move identically or symmetrically, there were signs having only one hand at the location above the eyebrow ridge, and the other below the eyebrow ridge. They were coded as citation forms. Only signs with both hands located below the eyebrow ridge were coded as non-citation forms. A maximum of three tokens with the same lexical item (type) were coded in order to reduce potential lexical effects as suggested in the previous studies (Lucas et al., 2001; Schembri et al., 2009).

In accordance with the previous studies, a similar coding scheme was used to code the linguistic factors. These factors are as follows:

1. Grammatical function of target sign: noun (e.g., GOVERNMENT, RABBIT), verb (e.g., KNOW, THINK, UNDERSTAND), adjective (e.g., BLACK, CRAZY, DIZZY), adverb (e.g., TOMORROW), grammatical item (e.g., SORRY)

2. Change in hand dominance: no (no change in hand dominance before and after the target sign), before (change in hand dominance between the target sign and the sign before ), after (change in hand dominance between the target sign and the sign after), both (change in hand dominance between the target sign and both the sign before and after) 
3. Preceding segment: sign, pause (target is the first sign $)^{18}$

4. Location of preceding segment: head, body, not applicable (change in hand dominance before the target sign)

5. Contact of preceding segment: no (no contact), head, hand, body, not application (change in hand dominance before the target sign)

6. Following segment: sign, pause (target is the last sign)

7. Location of following segment: same codes as for location of preceding segment, not applicable (change in hand dominance after the target sign)

8. Contact of following segment: same codes as for contact of preceding segment, not applicable (change in hand dominance after the target sign)

9. Lexical frequency: high, low (Based on the frequency distributions in Table 4.3, a qualitative decision was made to contrast the 8 most common signs comprising half the total data set with all others.)

\footnotetext{
${ }^{18}$ There was a small number of meaningful gestures in the preceding/following segments. They were coded as sign.
} 


\begin{tabular}{|c|c|c|c|c|c|c|}
\hline & Target signs & $\begin{array}{l}\text { No. of tokens } \\
\text { in dataset }\end{array}$ & $\%$ of tokens in dataset & Cumulative \% & No. of cf & $\%$ of cf \\
\hline 1. & NOT-UNDERSTAND & 93 & $11.63 \%$ & $11.63 \%$ & 52 & $55.91 \%$ \\
\hline 2. & KNOW & 88 & $11.00 \%$ & $22.63 \%$ & 62 & $70.45 \%$ \\
\hline 3 . & THINK & 68 & $8.50 \%$ & $31.13 \%$ & 52 & $76.47 \%$ \\
\hline 4. & REMEMBER & 40 & $5.00 \%$ & $36.13 \%$ & 37 & $92.50 \%$ \\
\hline 5. & UNDERSTAND & 36 & $4.50 \%$ & $40.63 \%$ & 30 & $83.33 \%$ \\
\hline 6. & GOVERNMENT & 35 & $4.38 \%$ & $45.00 \%$ & 33 & $94.29 \%$ \\
\hline 7. & LEARN & 34 & $4.25 \%$ & $49.25 \%$ & 33 & $97.06 \%$ \\
\hline 8. & \#-DAY & 33 & $4.13 \%$ & $53.38 \%$ & 29 & $87.88 \%$ \\
\hline 9. & DIFFICULT & 23 & $2.88 \%$ & $56.25 \%$ & 16 & $69.57 \%$ \\
\hline 10. & HSD & 23 & $2.88 \%$ & $59.13 \%$ & 11 & $47.83 \%$ \\
\hline 11. & TROUBLESOME & 22 & $2.75 \%$ & $61.88 \%$ & 21 & $95.45 \%$ \\
\hline 12. & LSD & 21 & $2.63 \%$ & $64.50 \%$ & 6 & $28.57 \%$ \\
\hline 13. & TOMORROW & 20 & $2.50 \%$ & $67.00 \%$ & 18 & $90.00 \%$ \\
\hline 14. & FORGET & 18 & $2.25 \%$ & $69.25 \%$ & 11 & $61.11 \%$ \\
\hline 15. & LUCKY & 16 & $2.00 \%$ & $71.25 \%$ & 13 & $81.25 \%$ \\
\hline 16. & FUTURE & 13 & $1.63 \%$ & $72.88 \%$ & 9 & $69.23 \%$ \\
\hline 17. & HIU-CHONG & 11 & $1.38 \%$ & $74.25 \%$ & 9 & $81.82 \%$ \\
\hline 18. & BLACK & 10 & $1.25 \%$ & $75.50 \%$ & 6 & $60.00 \%$ \\
\hline 19. & cow & 10 & $1.25 \%$ & $76.75 \%$ & 6 & $60.00 \%$ \\
\hline 20. & RESEARCH & 10 & $1.25 \%$ & $78.00 \%$ & 9 & $90.00 \%$ \\
\hline 21. & SORRY & 9 & $1.13 \%$ & $79.13 \%$ & 8 & $88.89 \%$ \\
\hline 22. & KNOW-WHAT-TO-DO & 8 & $1.00 \%$ & $80.13 \%$ & 8 & $100.00 \%$ \\
\hline 23. & CLEVER & 7 & $0.88 \%$ & $81.00 \%$ & 5 & $71.43 \%$ \\
\hline 24. & HEAD & 7 & $0.88 \%$ & $81.88 \%$ & 6 & $85.71 \%$ \\
\hline 25. & HOT & 7 & $0.88 \%$ & $82.75 \%$ & 7 & $100.00 \%$ \\
\hline 26. & MEDICAL & 7 & $0.88 \%$ & $83.63 \%$ & 7 & $100.00 \%$ \\
\hline 27. & NOT-KNOW & 7 & $0.88 \%$ & $84.50 \%$ & 4 & $57.14 \%$ \\
\hline 28. & CRAZY & 6 & $0.75 \%$ & $85.25 \%$ & 5 & $83.33 \%$ \\
\hline 29. & DREAM & 6 & $0.75 \%$ & $86.00 \%$ & 5 & $83.33 \%$ \\
\hline 30. & MONEY-MINDED & 6 & $0.75 \%$ & $86.75 \%$ & 6 & $100.00 \%$ \\
\hline 31. & RABBIT & 6 & $0.75 \%$ & $87.50 \%$ & 6 & $100.00 \%$ \\
\hline 32. & SICK & 6 & $0.75 \%$ & $88.25 \%$ & 4 & $66.67 \%$ \\
\hline 33. & TALK-NONSENSE & 6 & $0.75 \%$ & $89.00 \%$ & 4 & $66.67 \%$ \\
\hline 34. & FREE-FROM-DUTY & 5 & $0.63 \%$ & $89.63 \%$ & 3 & $60.00 \%$ \\
\hline 35. & INEXPLICABLE & 5 & $0.63 \%$ & $90.25 \%$ & 3 & $60.00 \%$ \\
\hline 36. & PRACTICE & 5 & $0.63 \%$ & $90.88 \%$ & 4 & $80.00 \%$ \\
\hline 37. & MEMORY-FADING & 4 & $0.50 \%$ & $91.38 \%$ & 4 & $100.00 \%$ \\
\hline 38. & POLICE & 4 & $0.50 \%$ & $91.88 \%$ & 4 & $100.00 \%$ \\
\hline 39. & ALL-DONE & 3 & $0.38 \%$ & $92.25 \%$ & 3 & $100.00 \%$ \\
\hline 40. & CONSERVATIVE & 3 & $0.38 \%$ & $92.63 \%$ & 2 & $66.67 \%$ \\
\hline 41. & DIZZY & 3 & $0.38 \%$ & $93.00 \%$ & 3 & $100.00 \%$ \\
\hline 42. & EVERYDAY & 3 & $0.38 \%$ & $93.38 \%$ & 3 & $100.00 \%$ \\
\hline 43. & HABIT & 3 & $0.38 \%$ & $93.75 \%$ & 2 & $66.67 \%$ \\
\hline 44. & INVENT & 3 & $0.38 \%$ & $94.13 \%$ & 3 & $100.00 \%$ \\
\hline 45. & REVENGEFUL & 3 & $0.38 \%$ & $94.50 \%$ & 3 & $100.00 \%$ \\
\hline 46. & TOO-NERVOUS & 3 & $0.38 \%$ & $94.88 \%$ & 3 & $100.00 \%$ \\
\hline 47. & HOPE & 2 & $0.25 \%$ & $95.13 \%$ & 1 & $50.00 \%$ \\
\hline 48. & IMAGINE & 2 & $0.25 \%$ & $95.38 \%$ & 2 & $100.00 \%$ \\
\hline 49. & INSANE & 2 & $0.25 \%$ & $95.63 \%$ & 2 & $100.00 \%$ \\
\hline 50. & KOREA & 2 & $0.25 \%$ & $95.88 \%$ & 2 & $100.00 \%$ \\
\hline 51. & MATURE & 2 & $0.25 \%$ & $96.13 \%$ & 2 & $100.00 \%$ \\
\hline 52. & NARCOSIS & 2 & $0.25 \%$ & $96.38 \%$ & 2 & $100.00 \%$ \\
\hline 53. & NO-REASON & 2 & $0.25 \%$ & $96.63 \%$ & 2 & $100.00 \%$ \\
\hline 54. & NOTHING-IN-HEAD & 2 & $0.25 \%$ & $96.88 \%$ & 2 & $100.00 \%$ \\
\hline 55. & PHILIPPINES & 2 & $0.25 \%$ & $97.13 \%$ & 2 & $100.00 \%$ \\
\hline 56. & POWERFUL & 2 & $0.25 \%$ & $97.37 \%$ & 1 & $50.00 \%$ \\
\hline 57. & SUN & 2 & $0.25 \%$ & $97.62 \%$ & 0 & $0.00 \%$ \\
\hline 58. & UNLUCKY & 2 & $0.25 \%$ & $97.87 \%$ & 2 & $100.00 \%$ \\
\hline 59. & BEAR-IN-MIND & 1 & $0.13 \%$ & $98.00 \%$ & 1 & $100.00 \%$ \\
\hline 60. & 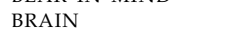 & 1 & $0.13 \%$ & $98.12 \%$ & 1 & $100.00 \%$ \\
\hline 61. & COW-HEAD & 1 & $0.13 \%$ & $98.25 \%$ & 1 & $100.00 \%$ \\
\hline 62. & CREATE & 1 & $0.13 \%$ & $98.37 \%$ & 0 & $0.00 \%$ \\
\hline 63. & DISNEYLAND & 1 & $0.13 \%$ & $98.50 \%$ & 1 & $100.00 \%$ \\
\hline 64. & GOOD-MEMORY & 1 & $0.13 \%$ & $98.62 \%$ & 1 & $100.00 \%$ \\
\hline 65. & HARD-UP & 1 & $0.13 \%$ & $98.75 \%$ & 1 & $100.00 \%$ \\
\hline 66. & HOW-MANY-DAYS & 1 & $0.13 \%$ & $98.87 \%$ & 1 & $100.00 \%$ \\
\hline 67. & KNOWLEDGE & 1 & $0.13 \%$ & $99.00 \%$ & 1 & $100.00 \%$ \\
\hline 68. & NOON & 1 & $0.13 \%$ & $99.12 \%$ & 1 & $100.00 \%$ \\
\hline 69. & NURSE & 1 & $0.13 \%$ & $99.25 \%$ & 1 & $100.00 \%$ \\
\hline 70. & PLEASE & 1 & $0.13 \%$ & $99.37 \%$ & 0 & $0.00 \%$ \\
\hline 71. & REGRET & 1 & $0.13 \%$ & $99.50 \%$ & 1 & $100.00 \%$ \\
\hline 72. & RUDE & 1 & $0.13 \%$ & $99.62 \%$ & 1 & $100.00 \%$ \\
\hline 73. & STUPID & 1 & $0.13 \%$ & $99.75 \%$ & 1 & $100.00 \%$ \\
\hline 74. & SUGGEST & 1 & $0.13 \%$ & $99.87 \%$ & 1 & $100.00 \%$ \\
\hline \multirow[t]{2}{*}{75.} & THE-ONLY-ONE & 1 & $0.13 \%$ & $100.00 \%$ & 1 & $100.00 \%$ \\
\hline & Total & 800 & & & 613 & $76.63 \%$ \\
\hline
\end{tabular}

Table 4.3: List of all target signs and their frequency 
In addition to the above linguistic factors, I also coded for handshape markedness and handedness of target signs. The seven most unmarked handshapes (i.e., A, B, C, S, O, 1, 5) and three related, less marked handshapes were coded as unmarked. ${ }^{19}$ They are illustrated in Figure 4.19. All other handshapes were coded as marked. Deuchar (1981, p. 112) observed that phonological deletion seemed to be more common when a sign occurs with an unmarked handshape. By analogy, then, it seems possible that in signs using the unmarked handshapes might be more likely to be subject to location drop. For handedness, I coded whether the target sign was one-handed or two-handed. Since two-handed signs have offered phonologists essential clues in describing sign language phonology (Brentari, 1998), they may possibly shed light on phonological variation in some way. However, two-handed target signs were far less common than one-handed ones in the data. Moreover, only a handful of participants produced two-handed target signs. In view of this biased dataset, handedness was not considered as a potential linguistic factor in the analysis.

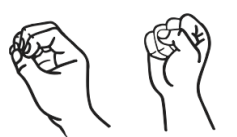

O S

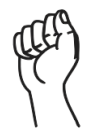

A

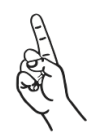

1

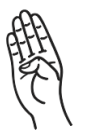

B

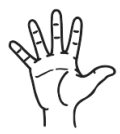

5

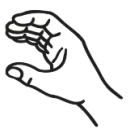

C
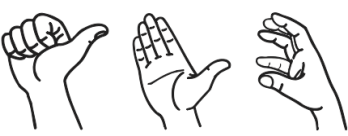

related less marked handshapes

Image courtesy of NZSL Online by Deaf Studies Research Unit, Victoria University of Wellington

Figure 4.19: Handshapes coded as unmarked

\footnotetext{
${ }^{19}$ Marked handshapes are structurally more complex, whereas unmarked or less marked handshapes are more simple and easy to articulate. Unmarked handshapes acquired earlier and children tend to substitute marked handshapes with unmarked ones. Also, unmarked handshapes are more frequent and are used for non-dominant hand in two-handed signs (Brentari, 1998).
} 
The coding scheme for social factors is the same as the one adopted for DEAF/HEARING variables and is outlined in the next section about interview data.

\subsubsection{Interview data}

For the interview data, each video clip of the 40 selected participants was segmented and annotated according to themes. Based on the information I gathered in the interviews, I examined the social network of each participant with an attempt to generate a social network index for further analysis. However, it turned out to be unsuccessful for the reason that diverse responses were given to the same question, which made it difficult to quantify in an impartial and convincing way. It would be more constructive and meaningful to create a standardized form, and the interview data is a good reference for creating such a social network measurement form. Consequently, the idea of analyzing the possible effect of social network in the phonological variables was discarded.

When viewing the interview data, it was found that a number of participants were working in deaf-related agencies. In such work environment, being deaf means the individual would be viewed as a sign language model for their hearing colleagues, customers, or even deaf children. Some participants emphasized their change in view about sign language after working in CSLDS. Considering this may have potential effect on their signing, I also coded whether the participant is working in a deaf related environment or not. This factor group overlaps with whether or not the participant was a sign language teacher, a potential social factor. 
However, there were only five sign language teachers, i.e., less than $10 \%$ of the participants. Therefore, sign language teacher was not coded for analysis.

Personal information given on the short demographic profile by each participant was used to code for other social factors. In cases where the information on the profile sheet was not clear or different from that provided in the interview, information from the latter was used for coding. All social factors coded are listed below:

1. Age group: younger (18-34), middle (35-49), older (50 and above)

2. Gender: male, female

3. School attended: CMS, HSD, LSD, OMS, VSD (see Section 4.3)

4. Region: HKI, KLE, KLW, NTE, NTW (see Section 4.3)

5. Educational level: low (Primary 6 or below), average (from some secondary to vocational training), high (diploma or above)

6. language background: HKSL (have deaf parents), other (have hearing parents)

7. Age entered deaf school: 7 or before, after 7

8. Work environment: deaf (work at CSLDS, deaf school, deaf organization, or deaf restaurant), non-deaf (work in non-deaf related settings $)^{20}$

\footnotetext{
${ }^{20}$ Participants who have deaf colleagues in their work environment were coded as nondeaf (work in non-deaf related settings).
} 


\subsection{Data analysis}

The lexical data exported from ELAN was checked and then imported into $R$, an open-source and user-extendable statistical software environment for cross-tabulation, graph plotting, and statistical testing. For each of the 51 lexical items, variants and social factors were cross-tabulated to show frequency and percentage. Patterns of distribution in contingency tables revealed whether any potential associations existed. When a correlation was observed, it was further tested for significance by using the Fisher's Exact test or the Chi-square test.

For binary variables in compound, 'citation form,' number handedness (lexical variation), and location variable (phonological variation), random forest analysis and first series of regression were conducted in R. The former helped to find out which factors are more important than others, while the latter showed the effects of the linguistic and social factors and which factor groups could be collapsed into fewer levels. Based on the information gathered, relevant factor groups were then selected to perform multivariate logistic regression in Rbrul (Johnson, 2009). This allows the analyst to control for some of the distributional imbalances across the factors that have been coded for present in natural speech/signing.

Like the original variable rule program VARBRUL, and later GoldVarb, Rbrul is designed for analyzing sociolinguistic data and aimed to improve the previous programmes. One advantage in using Rbrul is that it presents results in both factor weights and log odds. The former is familiar to sociolinguists and allows cross-linguistic comparison with the previous studies which use VARBRUL and GoldVarb; the latter is commonly used in 
statistics and is more comprehensible in fields like psycholinguistics. Another advantage of Rbrul is its capability to fit mixed-effects modelling, taking both between-group effects and within-group effects into account for analysis. However, random effects in Rbrul assume a normal distribution. By virtue of the nature of language acquisition among HKSL users, it is very likely that the signers are non-normally distributed in terms of the variables examined. Moreover, small sample size and imbalanced number of tokens may affect the result of mixed-effects modelling (Moineddin, Matheson, \& Glazier, 2007). Taking these considerations, mixed-effects modelling was used in the compound and 'citation form' datasets with lexical items as random effect; fixed-effects modelling was used in the number handedness and location variable datasets. In addition, conditional inference trees were also used to further explore the combined effect of the social factors. Conditional inference trees are "a method for regression and classification based on binary recursive partitioning" (Levshina, 2015, p. 291). They are useful in analyzing data with complex interactions, small sample sizes and a large number of predictors. The reason for using conditional inference trees to complement the Rbrul analysis is that multiple regression modelling may be problematic when there are complex interactions between predictors within a small sample. Since both approaches have their advantages and disadvantages, some researchers suggested using the two in tandem (Eddington, 2010).

For the first phonological variables, movement pattern in the signs DEAF and HEARING, a descriptive analysis was conducted. This is because of the small data size (580 tokens of DEAF and 365 tokens of HEARING), and the imbalance in the number of tokens from each individual (2 to 52 tokens for DEAF and 1 to 35 tokens for HEARING). R was also used to calculate 
and visualize the distribution of variants by various linguistic factors and social factors. When a potential correlation was detected, a Fisher Exact test or Chi-square test was used to test for statistical significance.

\subsection{Multicollinearity and collinearity}

Collinearity refers to the linear correlation between two variables, while multicollinearity appears when more than two variables are correlated with each other. It should be noted that in my dataset, particularly the smaller dataset used to analyze phonological variables, many of the social factor groups are non-orthogonal, i.e., they are correlated with each other to a certain extent. For instances, many participants who are living in NTW went to LSD and those living in HKI went to CMS; it happened that all participants with deaf parents had attended HSD; only older signers have low educational level; no participants from CMS work in a deafrelated organization. Therefore, the issue of multicollinearity would arise when performing a regression analysis with those social variables. Even if the presence of multicollinearity is unavoidable, it is essential to estimate the degree of multicollinearity and its potential impact on regression analysis, due to the small sample size in this study (Baguley, 2012).

In order to detect if there are any problems with multicollinearity, the variance inflation factor (VIF) and tolerance of the social factor groups were calculated. Table 4.4 shows the VIF and tolerance of the three subsamples (i.e., compound signs, number handedness, and 'citation form') in the dataset $(\mathrm{N}=65)$ for lexical analysis. The VIF values of school in all three sub-samples and that of education in the number handedness sub-sample 
are greater than 10 and their tolerance values are less than 0.1 , which indicate a problem with multicollinearity of the independent variables (Hair, Black, Babin, Anderson, \& Tatham, 2006; Menard, 2002). ${ }^{21}$ However, for the reason that school is one of the key factors in this study and research has suggested that school is a better predictor of variant choice than where one lives (Quinn, 2010; Stamp et al., 2014), it seemed more appropriate to eliminate other variables like region, in order to reduce the effect of multicollinearity. After removing the factor group region (the second columns in Tables 4.4 and 4.5), all three VIF values for school have largely reduced and the tolerances have risen. However, only the compound signs subsample has dropped below 10, the other two sub-samples are still problematic, particularly the number handedness sample where the VIF values of school and education are 24.672 and 13.039 respectively. For the other datasets $(\mathrm{N}=40)$ for phonological variation analysis (i.e., DEAF/HEARING variable and location variable), the problem of multicollinearity is also observed (see Table 4.5). Consequently, region was excluded in the Rbrul analysis and some social factor groups were combined (see Chapter 5 and 7). By excluding one factor group, it does not mean that the problem of multicollinearity is resolved, but it offers a remedy to mitigate the effect.

\footnotetext{
${ }^{21}$ Although the rule of thumb for VIF is commonly either 4 or 10 , some researchers may consider a VIF greater than 2.5 to be a point for concern (e.g., Glynn, 2014). Indeed, these rules of thumb are not without challenge (O'Brien, 2007).
} 


\begin{tabular}{lrrrrrr}
\hline & \multicolumn{5}{c}{ VIF(tolerance) } \\
\cline { 2 - 7 } Social factor group & \multicolumn{2}{c}{ compound signs } & \multicolumn{2}{c}{ number handedness } & \multicolumn{2}{c}{ 'citation form' } \\
\hline Age & $3.511(0.285)$ & $2.808(0.356)$ & $4.225(0.237)$ & $3.167(0.316)$ & $3.863(0.259)$ & $3.160(0.316)$ \\
Education & $8.289(0.121)$ & $6.401(0.156)$ & $14.669(0.068)$ & $13.039(0.077)$ & $7.698(0.130)$ & $6.239(0.160)$ \\
Gender & $1.322(0.757)$ & $1.247(0.802)$ & $1.317(0.759)$ & $1.243(0.805)$ & $1.315(0.760)$ & $1.255(0.797)$ \\
Language background & $2.042(0.490)$ & $1.821(0.549)$ & $1.553(0.644)$ & $1.339(0.747)$ & $2.262(0.442)$ & $1.957(0.511)$ \\
Region & $4.120(0.243)$ & - & $4.126(0.242)$ & - & $3.772(0.265)$ & - \\
School & $16.719(0.060)$ & $8.192(0.122)$ & $51.539(0.019)$ & $24.672(0.041)$ & $22.095(0.045)$ & $10.365(0.096)$ \\
Work environment & $2.297(0.435)$ & $2.069(0.483)$ & $1.918(0.521)$ & $1.851(0.540)$ & $2.178(0.442)$ & $1.976(0.506)$ \\
\hline
\end{tabular}

Table 4.4: VIF and tolerance of all social factor groups in the datasets for lexical analysis $(\mathrm{N}=65)$ (First column shows calculations with all social factor groups; second column shows calculations without region.)

\begin{tabular}{lcrrrrr}
\hline & \multicolumn{7}{c}{ VIF(tolerance) } \\
\cline { 2 - 7 } Social factor group & \multicolumn{2}{c}{ location variable } & \multicolumn{2}{c}{ DEAF variable } & \multicolumn{2}{c}{ HEARING variable } \\
\hline Age & $5.912(0.169)$ & $4.473(0.224)$ & $6.217(0.161)$ & $4.821(0.207)$ & $6.161(0.162)$ & $3.448(0.290)$ \\
Education & $5.045(0.198)$ & $5.007(0.200)$ & $3.454(0.290)$ & $2.304(0.434)$ & $1.729(0.579)$ & $1.512(0.661)$ \\
Gender & $1.183(0.845)$ & $1.076(0.930)$ & $1.284(0.779)$ & $1.166(0.857)$ & $2.023(0.494)$ & $1.335(0.749)$ \\
Language background & $1.978(0.506)$ & $2.083(0.480)$ & $3.437(0.291)$ & $2.729(0.366)$ & $2.005(0.499)$ & $2.011(0.497)$ \\
Region & $4.508(0.222)$ & - & $9.569(0.105)$ & - & $12.433(0.080)$ & - \\
School & $23.513(0.043)$ & $9.542(0.105)$ & $17.754(0.056)$ & $6.421(0.156)$ & $25.779(0.039)$ & $2.096(0.196)$ \\
Work environment & $1.888(0.530)$ & $1.816(0.551)$ & $2.939(0.340)$ & $2.758(0.363)$ & $1.929(0.518)$ & $2.051(0.488)$ \\
\hline
\end{tabular}

Table 4.5: VIF and tolerance of all social factor groups in the datasets for phonological analysis $(\mathrm{N}=40)$ (First column shows calculations with all social factor groups; second column shows calculations without region.)

There was another collinearity problem between two linguistic factors in the analysis of the second phonological variable: location drop. Initial analysis of location variation indicated that the name signs of two deaf schools exhibited a high proportion of non-citation variants. Due to the special connotation of deaf schools in the context of deaf conversation, a new factor group deaf school name sign was created to investigate its effects. However, this would cause overlapping between deaf school name sign and grammatical class for all deaf school name signs are proper nouns. Hence, a problem of collinearity between the two factor groups occurred and the VIF values of grammatical class and deaf school name sign are 27.61 and 13.56 
respectively. ${ }^{22}$ As mentioned earlier, a VIF greater than 10 indicates a serious multicollinearity problem and needs to be addressed. To avoid the problem, I combined the two factor groups to form a new group grammatical class $x$ deaf school name sign, which basically added the factor 'deaf school name sign' in the original grammatical class factor group. There are three forehead-located deaf school name signs: HSD, LSD, and HIU-CHONG (one of the OMS). There are 50 tokens of these signs and they are coded as 'deaf school name sign' under the new factor group accordingly.

${ }^{22}$ This calculation used the dataset that excluded all NAs and knockouts (respondents who produced only one variable), i.e., $\mathrm{N}=695$. 



\section{Chapter 5}

\section{Lexical variation}

\subsection{Overview}

This chapter reports and discusses the findings of the analysis of the lexical items elicited through picture naming. I first provide a general overview of the findings, concentrating on the extent of lexical variation and the distribution of separate variants and phonologically related subvariants in Sections 5.2 and 5.3. Section 5.4 presents the association between lexical variation and social factors, including school, age, and gender. Section 5.5 discusses the use of the four pairs of possible homonyms under investigation. The results of the analysis of the three sub-samples: compound signs, 'citation form,' and number handedness are detailed in Sections 5.6, 5.7, and 5.8. Synthesizing all the evidence from the findings, the issue of language change in progress is considered in Section 5.9. Lastly, Section 5.10 summarizes the discussion in this chapter. 


\subsection{Extent of lexical variation}

There were 3,400 lexical tokens in my dataset. Since the participants sometimes produced more than one variant, the dataset included 109 extra tokens. The distribution of these extra tokens across different categories is listed in Table 5.1. Excluding DEAF/HEARING and the four possible homonyms of colours, an average of 23 extra tokens were given in each of the four semantic domains. Country/region has the highest number of extra tokens, on average six per item within this category. This shows that many signers are aware of existing variants. They know and probably use more than one variant, especially the signs for country/region, in their daily conversation.

\begin{tabular}{lcc}
\hline Category (number of items) & Extra tokens & Average \\
\hline Colour (12) & 19 & 1.58 \\
Kinship (7) & 6 & 0.86 \\
Country/region (8) & 49 & 6.13 \\
Number (18) & 19 & 1.06 \\
Possible homonym (4) & 7 & 1.75 \\
DEAF/HEARING (2) & 9 & 4.50 \\
\hline Total & 109 & \\
\hline
\end{tabular}

Table 5.1: Number of extra tokens in each category

Because of the unique nature of number signs, most of the variants are phonologically related. The variants of number signs are then classified into one-handed or two-handed. The number of variants for each number sign is listed in Table 5.2. Numbers ten and below only have onehanded variants. The three instances of these numbers that occurred in the dataset (i.e., THREE, SEVEN and EIGHT) have two to four variants. For numbers over ten, on average, there are more one-handed variants (5.6) 
than two-handed variants (2.9). THIRTEEN has the highest number of variants (14), followed by ELEVEN and TWENTY-EIGHT (each has 11 variants). This may link to the fact that there are two possible handshapes for THREE and EIGHT, as well as the one-handed versions of ELEVEN (to be discussed in Section 5.4.1.4 on page 156). The two numbers with the fewest variants are FIFTEEN and HUNDRED, both of these numbers have six variants each. They both involve only unmarked handshapes in the formation of the sign (see Figure 4.19 on page 111 for handshapes illustrations).

\begin{tabular}{lccc}
\hline Number sign & One-handed & Two-handed & Total \\
\hline THREE & 4 & & 4 \\
SEVEN & 2 & & 2 \\
EIGHT & 3 & & 3 \\
\hline ELEVEN & 6 & 5 & 11 \\
TWELVE & 5 & 3 & 8 \\
THIRTEEN & 8 & 6 & 14 \\
FIFTEEN & 4 & 2 & 6 \\
SEVENTEEN & 5 & 3 & 8 \\
EIGHTEEN & 6 & 3 & 9 \\
TWENTY & 5 & 2 & 7 \\
TWENTY-ONE & 6 & 1 & 7 \\
TWENTY-TWO & 4 & 4 & 8 \\
TWENTY-THREE & 7 & 2 & 9 \\
TWENTY-FIVE & 6 & 2 & 8 \\
TWENTY-SEVEN & 5 & 2 & 7 \\
TWENTY-EIGHT & 9 & 2 & 11 \\
THIRTY & 5 & 3 & 8 \\
HUNDRED & 3 & 3 & 6 \\
Average no. of variants for numbers $>10$ & 5.6 & 2.9 & 8.5 \\
\hline
\end{tabular}

Table 5.2: Number of variants for each number sign

Excluding the number signs and the signs for DEAF and HEARING (which will be discussed in detail in Chapter 6 in the analysis of their phonological variation), there are 32 concepts for discussion. For these 32 concepts, a to- 
tal of 174 distinct variants were identified and 59 of these distinct variants have phonological subvariants (93 in total). This indicates that distinct lexical variants are more common than sublexical variants. Table 5.3 shows the number of lexical variants per concept. The average number of variants is 5.41. Eight of the stimulus concepts have four to six variants and another eight items have seven to nine variants. Three items produced ten or more variants and they were all colour terms (PURPLE, PINK and SILVER). The sign SILVER elicited the highest number of variants (17) and only one of them has subvariants. These three colour terms are less frequent signs compared with other items and this may be part of the explanation for their instability. When we consider all the colour terms, basic colours such as BLACK, WHITE and RED have fewer variants and they are mostly phonological subvariants. Derived colour terms tend to have more lexical variants like PURPLE, PINK and SILVER, as just mentioned.

\begin{tabular}{lc}
\hline Concepts with one variant & 4 \\
Concepts with two variants & 3 \\
Concepts with three variants & 6 \\
Concepts with four variants & 2 \\
Concepts with five variants & 1 \\
Concepts with six variants & 5 \\
Concepts with seven variants & 2 \\
Concepts with eight variants & 4 \\
Concepts with nine variants & 2 \\
Concepts with ten or more variants & 3 \\
\hline Total concepts & 32 \\
\hline
\end{tabular}

Table 5.3: Number of lexical variants per concept

Four of the 32 stimulus concepts produced only one lexical variant. Three of them were colour terms (YELLOW, RED and WHITE), the remaining one was FATHER. Among these four items, YELLOW elicited the same 
response from all the participants and each of the other three items produced one to four phonological subvariants. It is quite surprising to see all the older signers producing the 'modern form' of YELLOW because there is an 'old form' (separate variant) for YELLOW. The two variants are illustrated in Figure 5.1. The picture of the 'old form' was captured in the interview video where the signer was explaining his old name sign. This signer's surname is 黃 (WONG in English), and this character means yellow in Chinese. That is to say, the sign YELLOW can refer to both the colour yellow and someone's surname. It is worth noting that WONG is one of the five most common surnames in Hong Kong. This may probably increase its occurrences in HKSL discourse and contribute to the widespread use of the 'modern form.' As a result, the 'old form' has been phased out completely, at least in my dataset.

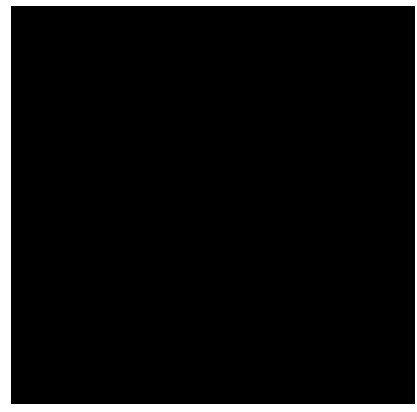

(a) Modern form

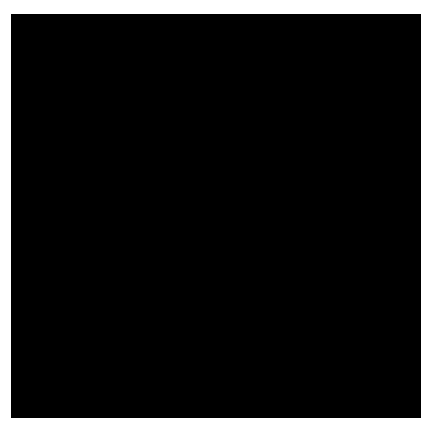

(b) Old form

Figure 5.1: Lexical variants of YELLOW

Lastly, only half of the stimulus items (16 out of 32 ) have a variant that is used by over half of the participants. All the seven kinship terms have a variant produced by more than half of the participants, as do three of the four possible homonyms. Only one-third (4 out of 12) of the colour terms and one-fourth (2 out of 8 ) of the country/region names have a variant given by more than $50 \%$ of the participants. Some of the most frequent 
variants are only used by $20 \%$ to $30 \%$ of the participants, such as PINK and BEIJING. Moreover, the signs for kinship terms appear to be more stable than the other semantic domains.

\subsection{Separate lexical variants and subvariants}

Since the sign YELLOW has no variation, the discussion here concentrates on 31 items, which consist of 2,067 tokens. For these 31 concepts, 173 separate lexical variants were recorded, with a total of 266 forms (including 93 phonological subvariants). The number of variants and subvariants for each of the 31 concepts is shown in Table 5.4. The numbers represent the separate lexical variants for each item and they are ordered from high to low occurrence in the dataset. The number in parentheses records the number of their subvariants. For example, BLACK has three lexical variants. Variant 1 is the most frequent sign and it has two subvariants, Variant 2 has no subvariant, Variant 3 is the least frequent sign and it has one subvariant. The total number of forms for BLACK is then six.

In the ASL study, the researchers noticed that the most frequently used lexical variants had the highest number of subvariants (Lucas et al., 2001). Thirty-one out of their 34 stimuli (i.e., 91\%) had subvariants for the most frequently used variants. Even for the items that had the highest number of lexical variants such as ARREST (11 distinct variants) and EARLY (13 distinct variants), only the one with the highest occurrence in the dataset had subvariants. On the other hand, the NZSL study reported another pattern. In their dataset, both more and less frequent variants have phonological subvariants (McKee \& McKee, 2011). Among the most frequently used 
variant for each of their 80 stimuli, only $32.5 \%$ had subvariants. My data seems to resemble the pattern reported in the ASL study. We can see from Table 5.4 that Variant 1 has the highest number of subvariants, that is 26 out of 31 signs $(84 \%)$. Similar to the ASL findings, the number of variants having subvariants drops gradually from high frequent variants to less frequent variants. For Variants 6 to 17, only two variants have subvariants. Also, the three variants with the highest number of subvariants are GREEN1, WHITE1 (four subvariants each) and KOREA1 (five subvariants). WHITE1 is the only lexical variant and GREEN1 and KOREA1 are the most frequently used variants.

Lucas et al. (2001) also noted that the high number of variants for certain lexemes may be linked to their classifier origins. ASL is a more established language and many nouns that originated in classifiers are completely lexicalized to represent a specific entity. On the other hand, the verbs in their sample were still in the process of lexicalization and they explained "because they represent actions and not things [they] may be more prone to variation" (p. 184). In my HKSL sample, one of the items with classifier origin is ORANGE(fruit), which produced 9 separate variants. ${ }^{1}$ Three of the nine variants have subvariants and five out of nine are compounds. Most of the variants refer to the different actions of eating an orange. This high number of variants and the use of compounds may suggest that lexicalization is in progress for the sign ORANGE(fruit) (Janzen, 2012).

\footnotetext{
${ }^{1}$ Comparison between the sign for fruit orange and the one for colour orange is discussed in Section 5.5 on page 194.
} 


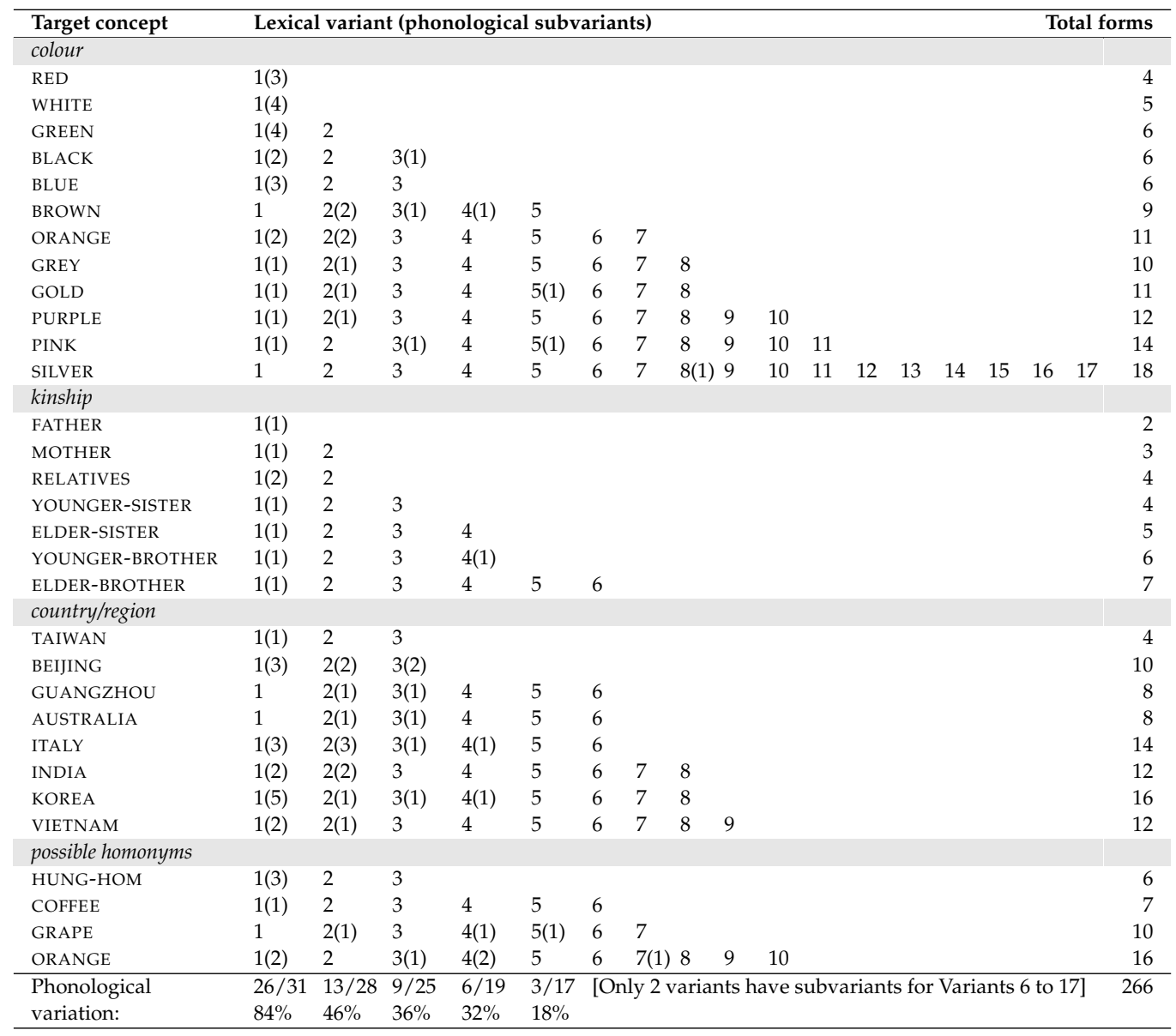

Table 5.4: Lexical variants and subvariants per concept

Finally, it is worth noting that 103 out of the 266 forms, i.e., near 39\%, were produced by only one signer in each case. Indeed in many cases, it is hard to differentiate idiosyncratic and phonological variations. As McKee \& McKee (2011, p. 503) explained:

The great many variants and subvariants recorded in this study confirms our impression (formed over twenty years of research) that the production of compositional elements of signs varies widely between individuals and age groups, to a degree more 
noticeable than a more established language such as ASL. Contributing to this variability are three main factors: (1) the youth and recently underground status of NZSL ...; (2) the scarcity of exposure to mature and native-language models during the acquisition experience of most NZSL users ...; and (3) extensive borrowing of foreign lexicon since the 1980s... (cf. Hoyer's $[2007,201]$ observation of an emerging sign language, also in a period of extensive borrowing, that "At all levels, idiolectal variation in Albanian Sign Language is more the rule than the exception.")

The three main causes leading to the great variability in NZSL described above may also be applied to the variations observed in HKSL. First, the emergence of HKSL can be traced back to the opening of the first deaf school in 1935. ${ }^{2}$ While the language was blossoming buttressed by the signing schools from the 1940s to 1960s, its development was hindered due to the oral only policy and the rise of inclusive education in the 1970s. It was not until around the mid-2000s that HKSL was officially allowed in deaf education. This history of deaf education has certainly interrupted the process of conventionalization of HKSL. Second, the phenomenon of deaf children learning sign language in deaf schools through their peers (rather than from their hearing parents or fluent adults in the community) is no exception in Hong Kong. The number of deaf teachers employed in deaf schools has been low since the full implementation of oral education, so has the number of deaf families. Third, borrowing from other sign lan-

\footnotetext{
${ }^{2}$ Nevertheless, it is possible that an earlier form of signing existed before deaf education began.
} 
guages is not uncommon and the pattern of lexical borrowing with signs for foreign countries and cities is quite widespread in signing communities. Like the country/region signs in the dataset, seven out of the eight signs elicited have a variant that is the country/region's own local sign. Among these seven borrowed signs, six of them have phonologically related subvariants.

In sum, distinct lexical variants are more common than phonological variants among the 31 concepts examined. The most frequently used variants tend to have the highest number of phonological subvariants, a pattern that mirrors the ASL findings. Some signs which have classifier origins appear to be in the process of lexicalization and exhibit a high degree of variation. Idiosyncratic variation seems to be quite common.

\subsection{Variation according to social factors}

This section reports the findings of lexical variation relating to three main social factors: school, age, gender. Target items of the four semantic categories (colour, kinship, country/region, and number) and four possible homonyms of colours are scrutinized, which consisted of 49 lexical items. Variants provided by only one signer are excluded and there are 266 variants including both distinct variants and phonologically related subvariants which are amenable to statistical analysis over the social factors. Fisher's Exact test is used to test the significance of the association between a certain variant and a social factor. Only items that show significance are discussed. 


\subsubsection{School}

School attended shows the strongest association with lexical variation in the dataset. Over $60 \%$ of the 49 stimulus items indicate a significant association between variants and schools. The total number of variants used by participants from different schools with the category breakdown is tabulated in Table 5.5. It should be noted that some participants went to two schools and the variants are counted twice in these cases. Slightly over half of these cases are those who went to VSD then CMS, and one-third are those who went to VSD then HSD (see Chapter 4 for details). Therefore, the findings of VSD and CMS may look analogous.

\begin{tabular}{lrrrrr}
\hline & CMS & HSD & LSD & OMS & VSD \\
\hline Colour & 52 & 53 & 48 & 40 & 52 \\
Kinship & 14 & 15 & 11 & 11 & 15 \\
Country/region & 40 & 48 & 36 & 37 & 40 \\
Number & 90 & 73 & 79 & 56 & 87 \\
\hline Total & 196 & 189 & 174 & 144 & 194 \\
\hline
\end{tabular}

Table 5.5: Total number of variants by categories and schools

As the figures show, the total number of variants produced by OMS participants is the smallest (144), which is nearly one-third less than the number produced by those who went to CMS, HSD, and VSD $(196,189$, and 194 respectively). This apparently links to the fact that the OMS participants learnt to sign from their teachers in signing schools. For the other participants who went to oral schools, different variants might have created by different cohorts as signing was not used as a medium of instruction as the OMS did. As a result, more diverse variants were produced by the participants from the oral schools as compared with those from signing schools. The LSD participants actually produced a smaller number 
of variants (174) than other participants from CMS/HSD/VSD, and this may be associated with the comparatively open attitude towards the use of signs in LSD. If the students were allowed to sign and even the teachers would use signs to teach in classrooms, it would help to spread the signs inside the school and pass them down from cohort to cohort.

One possible problem with using raw numbers (as in Table 5.5) is that the sample size from the schools differs. That is, there are 25 signers who went to HSD (compared to 12 who went to OMS), hence, if every signer produced roughly the same number of different variants, we would necessarily expect the raw numbers for HSD to be higher than they are for OMS. However, individuals did not produce the same number of variants at all. There was considerable variation in the number of variants produced by individuals who had attended the different schools. For this reason, it is not clear that transforming the variation in Table 5.5 into averages would provide a more accurate picture of the variation from the different schools.

Likewise, when we look at the number of variants given by only one single participant (see Figure 5.2), we can see that those from OMS and LSD produced the least, 25 and 26 respectively. The highest number of single user variants are produced by participants who went to HSD (44). ${ }^{3}$ This may be due to the long history of the school and their strict oral policy. Over the course of time, different cohorts created different variants in order to communicate with each other. For those who socialize mainly

\footnotetext{
${ }^{3}$ Again, transforming these raw numbers into averages over the number of participants who produced unique variants is subject to the same problems with individual outliers mentioned in the main text for Table 5.5. Nevertheless, if we convert the frequencies in Figure 5.2 to averages, the relative ranking of the schools does not change dramatically: $\mathrm{CMS}=2.5$ (average); $\mathrm{HSD}=3.67 ; \mathrm{LSD}=2.89 ; \mathrm{OMS}=2.5 ; \mathrm{VSD}=3.17$.
} 


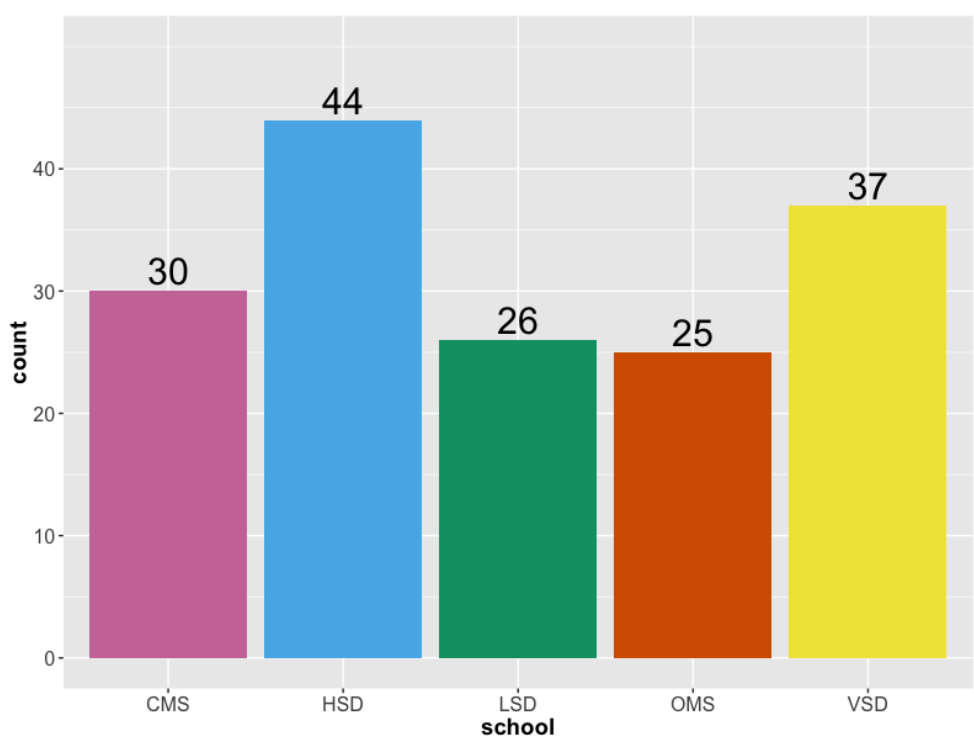

Figure 5.2: Number of unique variants given by a single participant by school (CMS $=12$ participants producing unique variants; $\mathrm{HSD}=12 ; \mathrm{LSD}=9 ; \mathrm{OMS}=10 ; \mathrm{VSD}=12$ )

with their classmates after they left the school, they would be more likely to keep the forms they learnt in school. For example, the unique variants GREEN9 and PURPLE9 (Figure 5.3) are given by one of the young participants from HSD whose social network is mainly his old classmates. When I asked my friends who went to HSD, they told me that these two forms were used in HSD when they were students there. After they left school and met other deaf people from other schools, they relinquished these forms and acquired the more commonly used forms. 


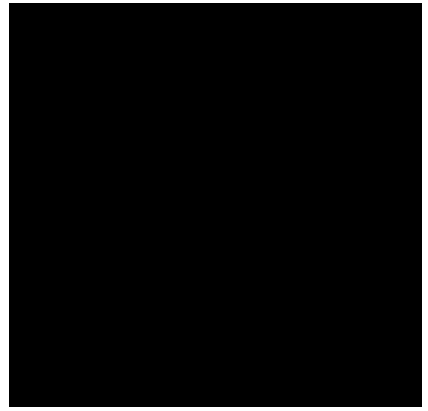

(a) GREEN9

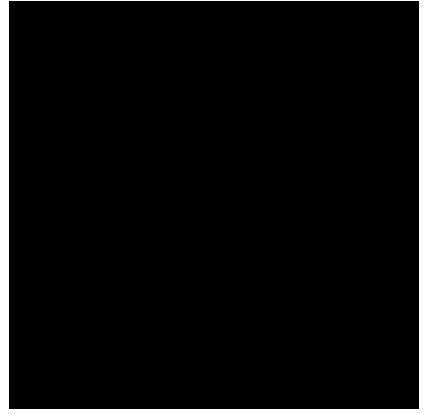

(b) PURPLE9

Figure 5.3: Examples of unique variants produced by single participant

\subsubsection{Colour terms and possible homonyms}

Nine out of the 12 colour terms appear to have school connected variants. ${ }^{4}$ Participants from different schools have different preferences for variant choice. The frequency distribution graphs of GREEN, PINK and GOLD demonstrate such a phenomenon (Figures 5.4, 5.5, and 5.7). In order to avoid double-counting in statistical calculation, VSD is not included in the findings reported hereafter. Those who went to OMS then CMS/HSD are classified as OMS; those who went to VSD then CMS or HSD are categorized as CMS and HSD respectively. One single participant who had only attended VSD is excluded. Hence, there were 14 participants from CMS; 24 from HSD; 14 from LSD; and 12 from OMS.

As Figure 5.4 shows, among the five phonologically related subvariants of GREEN, OMS tends to use GREENa, LSD favours GREENb, and HSD

\footnotetext{
${ }^{4}$ Due to the wide distribution and small numbers of variants in some items, some subvariants were grouped together and some minor variants were excluded to ensure fair numbers for statistical testing.
} 
prefers GREENC (see Figure 4.5 on page 91 for sign illustrations). But CMS seems to have no specific preference. Although all variants are used by CMS without strong inclination for a particular form, GREENd is exclusively produced by CMS.

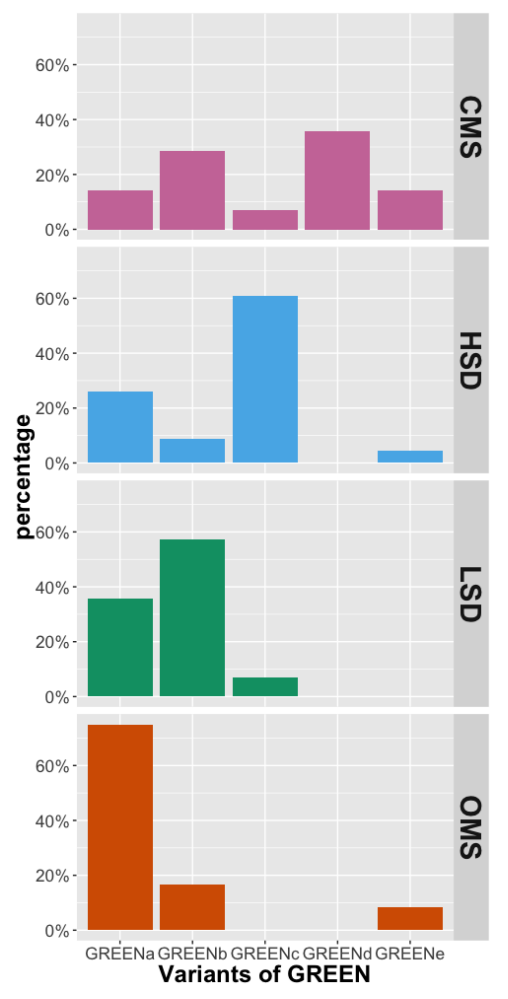

Figure 5.4: Percentage distribution by school: GREEN (Fisher's Exact test, $\mathrm{p}<0.0001)$

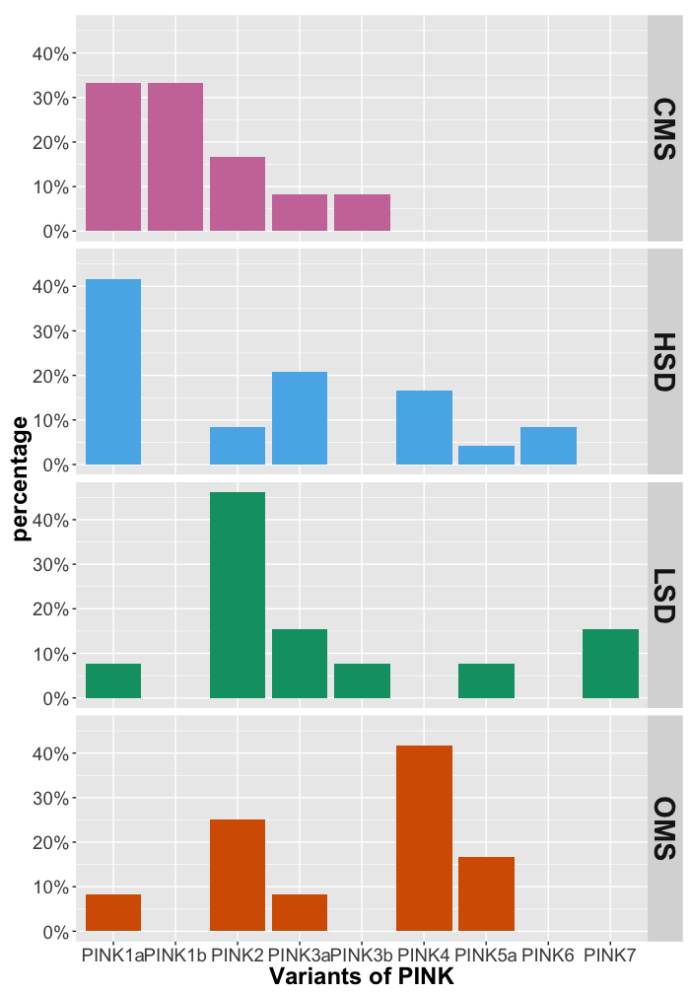

Figure 5.5: Percentage distribution by school: PINK (Fisher's Exact test, $\mathrm{p}<0.01$ )

A similar distribution is observed for PINK (see Figure 5.5). CMS and HSD tend to use PINK1, but LSD prefers PINK2 and OMS favours PINK4 (see Figure 5.6 for sign illustrations). While PINK1a is the most frequent form (around $24 \%$ of the participants used), its subvariant PINK $1 \mathrm{~b}$ is exclusively produced by CMS. In this case, the palm orientation is altered from palm facing out of the signer's body in PINK1a to palm facing in as in PINK1b. 


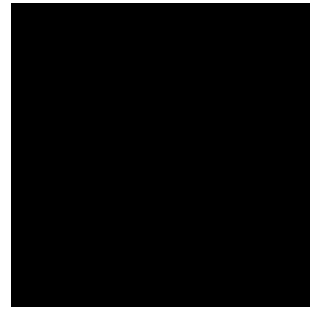

(a) PINK1a

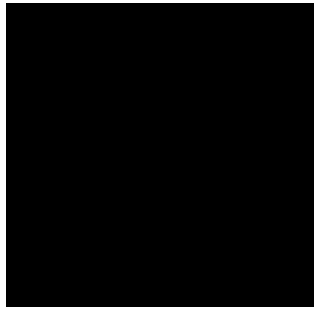

(b) PINK1b

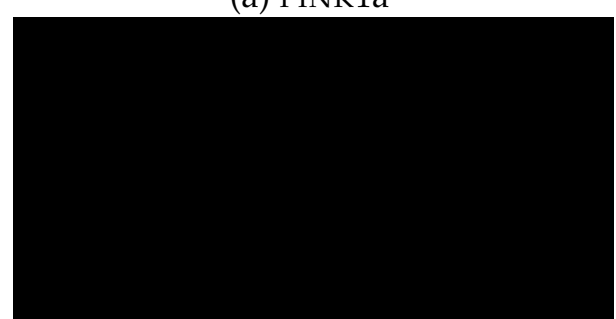

(c) PINK2 (d) PINK3

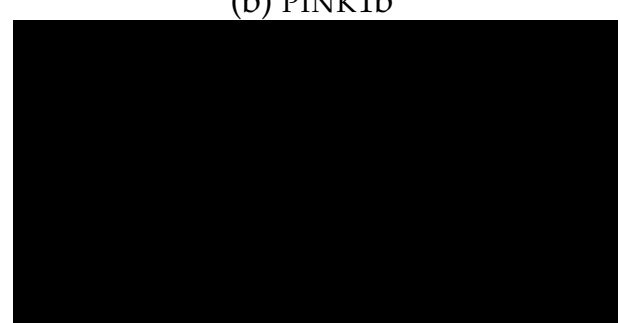

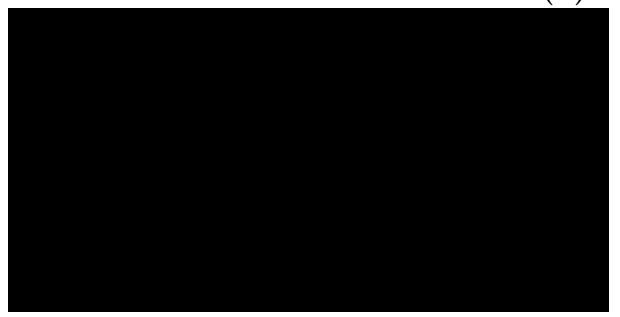

(e) PINK4

Figure 5.6: Major variants of PINK

With regard to GOLD, while GOLD1a is the most frequent form, none of the CMS participants use it. This variant is the favourite choice of HSD and OMS, LSD prefers its subvariant GOLD1b (see Figure 5.9). The CMS participants favour GOLD3, which has the same form as SILVER1 (see Figure 5.10). This explains why no LSD and OMS signers produced GOLD3 and only a few HSD signers used it. For non-CMS signers, this form refers to SILVER. Likewise, CMS does not produce as many SILVER1 as those from HSD or LSD (though it is still the most frequent among CMS signers). Many of them use this form for GOLD. 


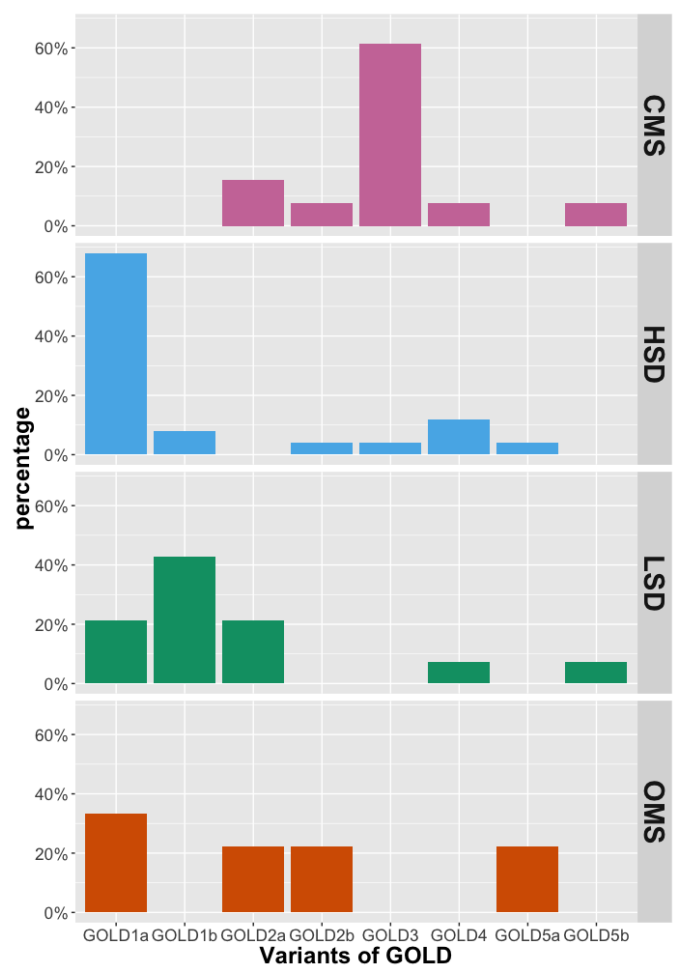

Figure 5.7: Percentage distribution by school: GOLD (Fisher's Exact test, $\mathrm{p}<0.0001$ )

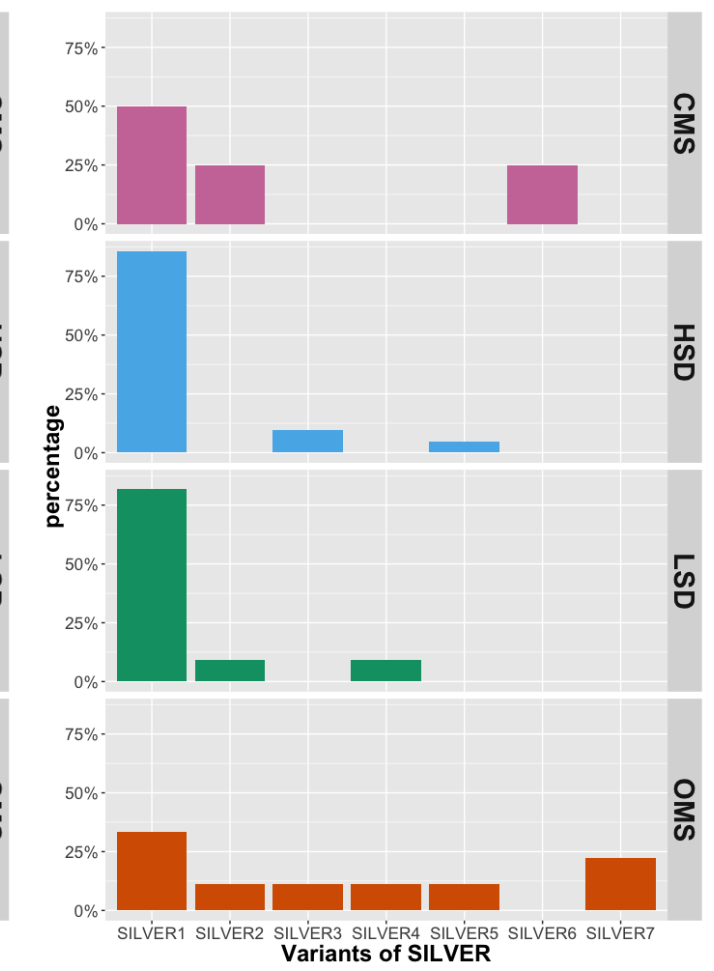

Figure 5.8: Percentage distribution by school: SILVER (CMS vs non-CMS, Fisher's Exact test, $\mathrm{p}<0.05$ )

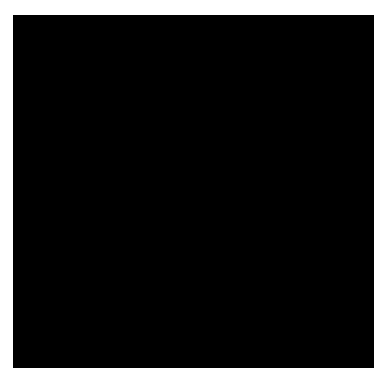

(a) GOLD1a

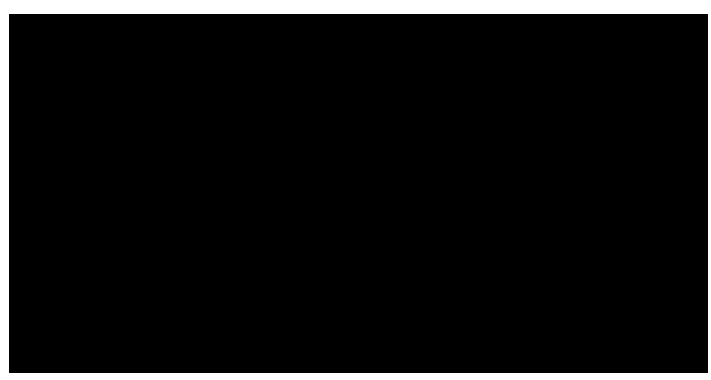

(b) GOLD1b

Figure 5.9: Phonological subvariants of GOLD 


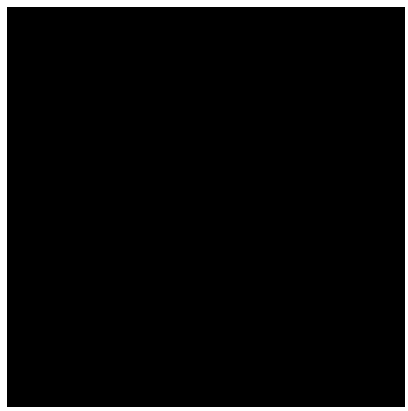

(a) GOLD3

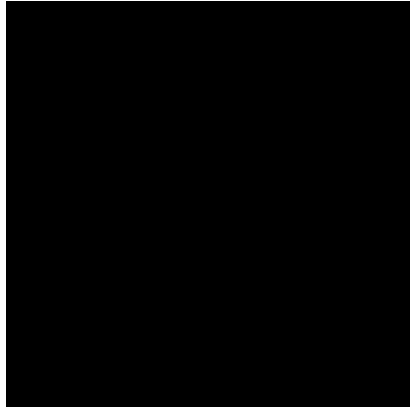

(b) SILVER1

Figure 5.10: Variants of GOLD and SILVER with the same form

In RED and ORANGE, HSD appears to have a distinct preference (see Figures 5.11 and 5.15). While all other schools favour the most frequent variant REDa (which is also the 'citation form' used in all HKSL teaching materials), HSD prefers REDb more than REDa (see Table 5.9 on page 173 for sign illustrations). Likewise, HSD tends to use ORANGE2a more than the others who favour ORANGE1a (Figure 5.13). In fact, it could be argued that ORANGE2a is a separate variant rather than a phonologically related variant of ORANGE2b. The former made use of one-hand to refer to the action of eating an orange, whereas the latter performs the action by twohand (see Figure 5.14). This two-handed form ORANGE2b is believed to be the original form of ORANGE2a in which one hand is dropped. Therefore, I grouped them as related subvariants. However, we can notice that the iconicity of eating an orange in ORANGE2a seems to be vanishing as compared to ORANGE2b. The handshape has evolved from a wedge-like handshape that holds an orange to a flat handshape. This may indicate a change in progress as the original two-handed form is mainly produced by OMS participants. 


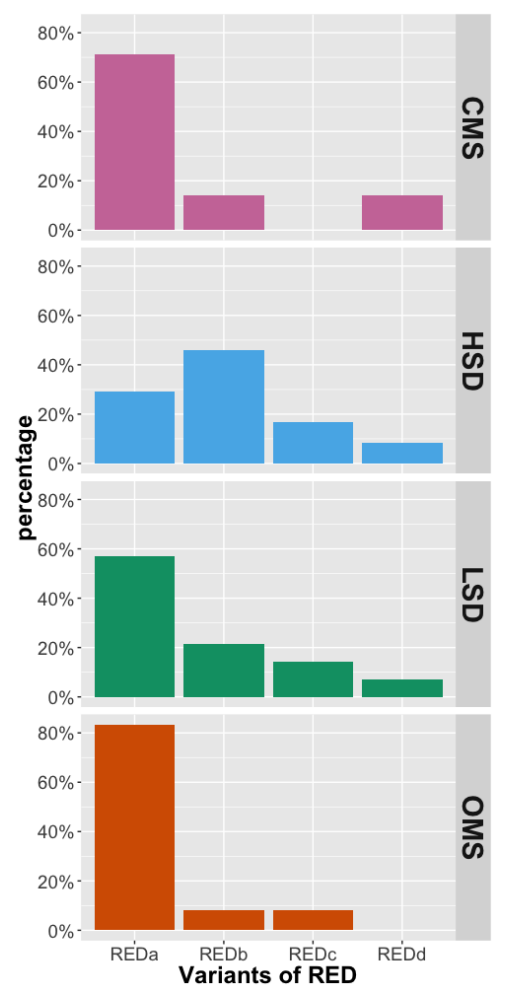

Figure 5.11: Percentage distribution by school: RED (HSD vs non-HSD, Fisher's Exact test, $\mathrm{p}<0.01$ )

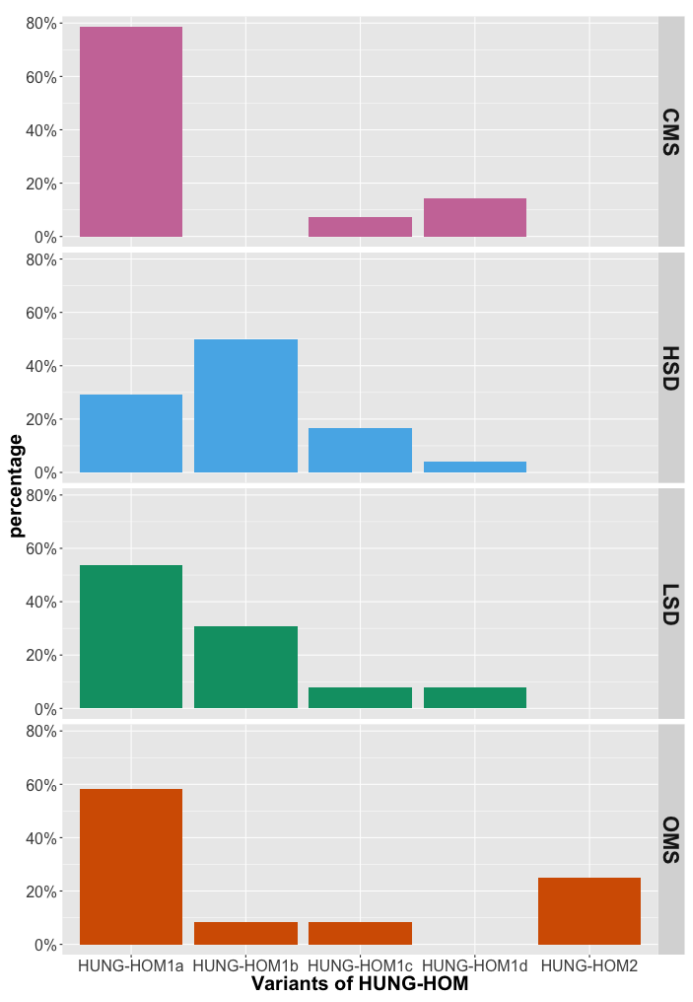

Figure 5.12: Percentage distribution by school: HUNG-HOM (HSD vs non-HSD, Fisher's Exact test, $\mathrm{p}<0.01$ )

Figure 5.13: The most frequent variant: ORANGE1a 


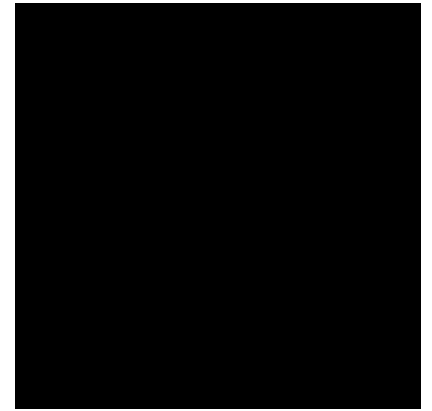

(a) ORANGE2a

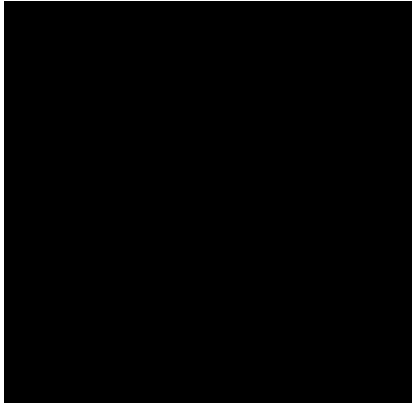

(b) ORANGE2b

Figure 5.14: Phonological subvariants of ORANGE2

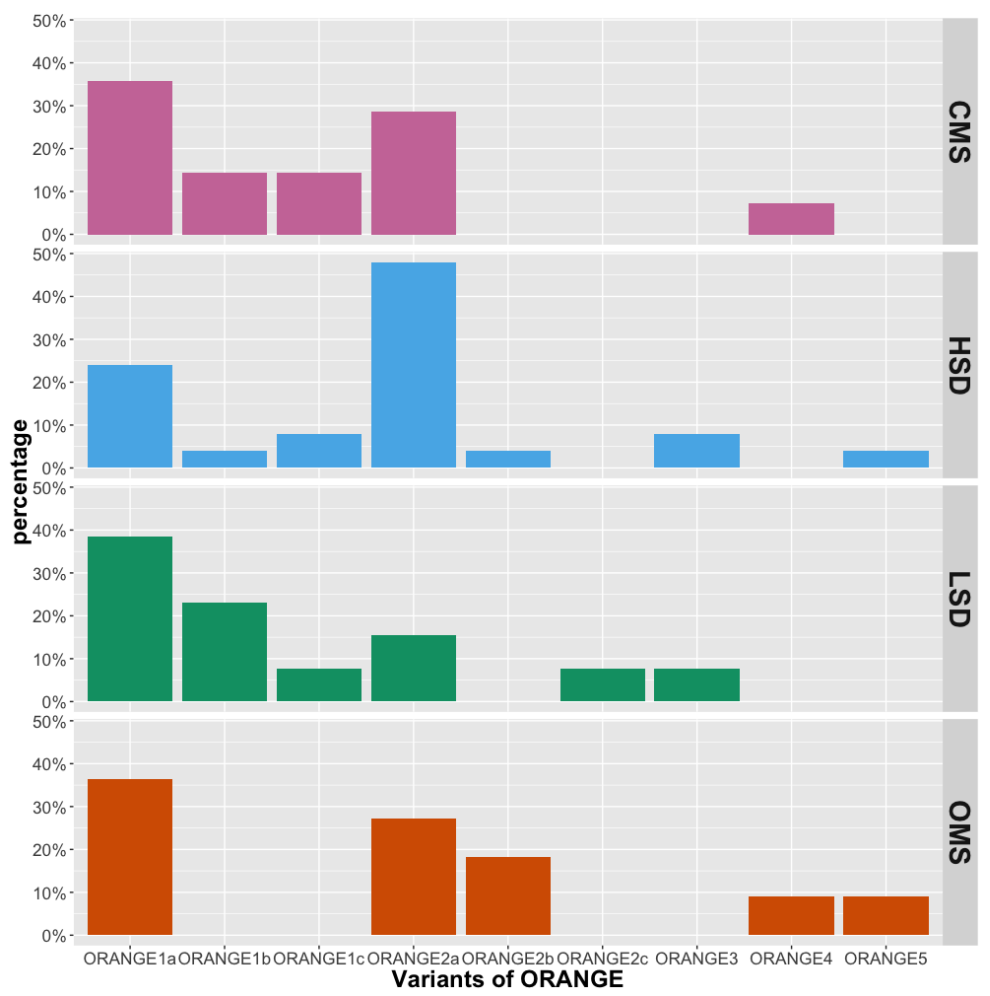

Figure 5.15: Percentage distribution by school: ORANGE (HSD vs non-HSD, Fisher's Exact test, $\mathrm{p}=0.05$ )

The frequency distribution of HUNG-HOM (local place name), a homonym of RED, demonstrates a similar pattern to RED (see Figures 5.11 and 5.12). HSD prefers HUNG-HOM1b (the same form as REDb) rather than the most 
frequent variant HUNG-HOM1a (the same form as REDa). OMS has a specific variant HUNG-HOM2 (Figure 5.16), which is a compound of RED and FERRY / PIER (the pier in Hung Hom is a well known landmark). On the other hand, the fruit ORANGE, a homonym of the colour ORANGE, does not exhibit any school association.

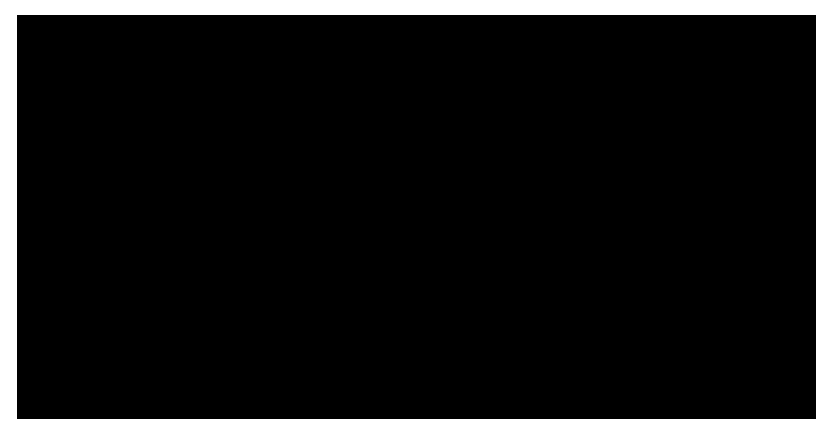

Figure 5.16: Variant used exclusively by OMS: HUNG-HOM2

Further, LSD prefers BLUEb and CMS favours PURPLE2a (see Figures 5.18 and 5.19). BLUEb is a subvariant which exhibits a different palm orientation from the most frequent form BLUEa (see Figure 4.3 on page 90 for sign illustrations); PURPLE2a (Figure 5.17b) is a separate variant which has a classifier origin describing grape. However, this preference is not found in the homonym GRAPE. This may indicate that CMS has separate forms for the two concepts to avoid homonymy. The use of homonyms will be discussed in Section 5.5. 


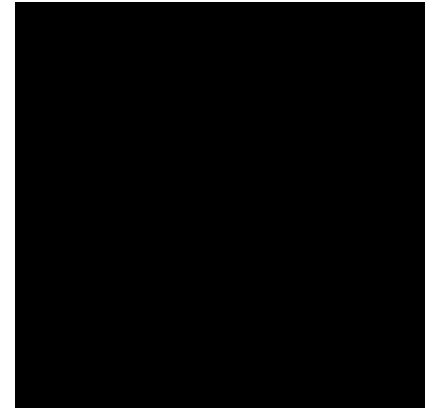

(a) PURPLE1a

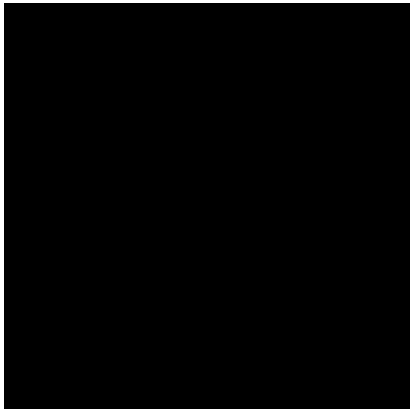

(b) PURPLE2a

Figure 5.17: Major variants of PURPLE

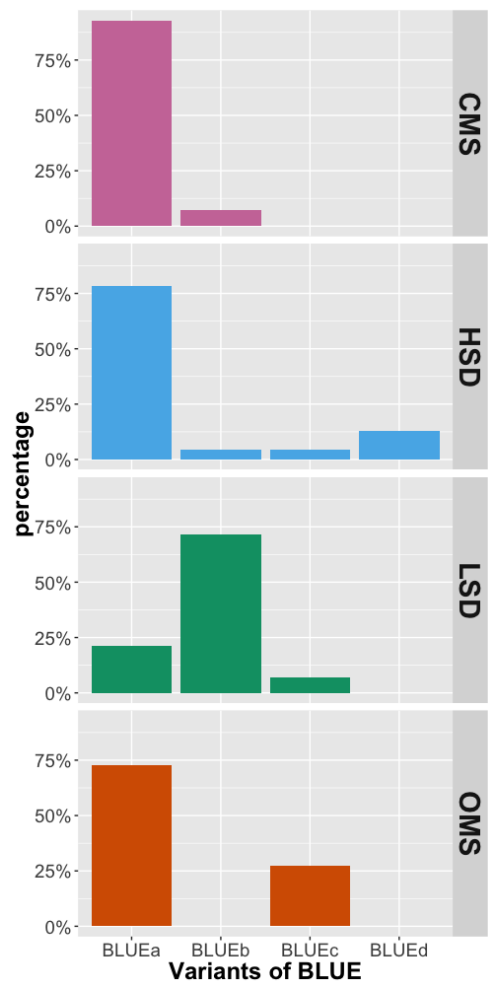

Figure 5.18: Percentage distribution by school: BLUE (LSD vs non-LSD, Fisher's Exact test, $\mathrm{p}<0.0001)$

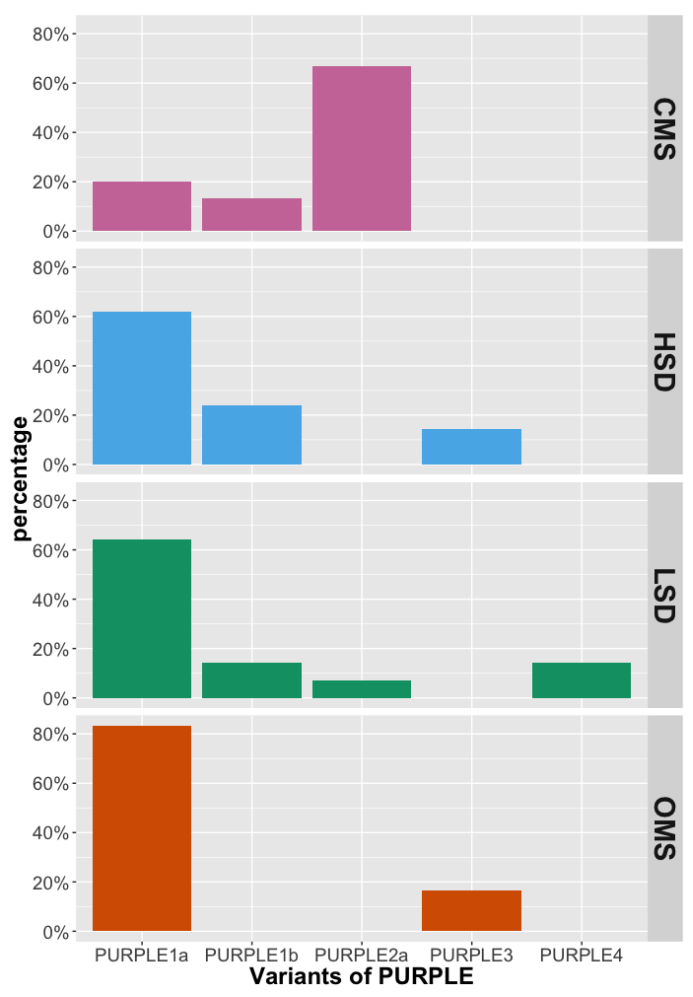

Figure 5.19: Percentage distribution by school: PURPLE (CMS vs non-CMS, Fisher's Exact test, $\mathrm{p}<0.0001)$

Lastly, it appears that there are school associated variants for CMS, LSD, and OMS in GREY (Figure 5.21). As we can see in Figure 5.20, GREY1a is the most frequent variant and signers from HSD, LSD, and OMS tend to 
use it the most. Nevertheless, GREY3 seems to link with LSD, with only one CMS user; and GREY4 is connected to OMS, with only one HSD user who has deaf parents studied at OMS. CMS prefers GREY2b, which is a subvariant of GREY2a and their only difference is handshape. GREY2a uses L-handshape (i.e., the first two fingers), while GREY $2 b$ makes use of the first three fingers. Both forms seem to be associated with CMS, as GREY2a is merely used by a few HSD signers and one LSD signer, and GREY2b is solely produced by CMS participants. Supposing that some variants are more school specific, they seem to find their way to reach signers from other schools.

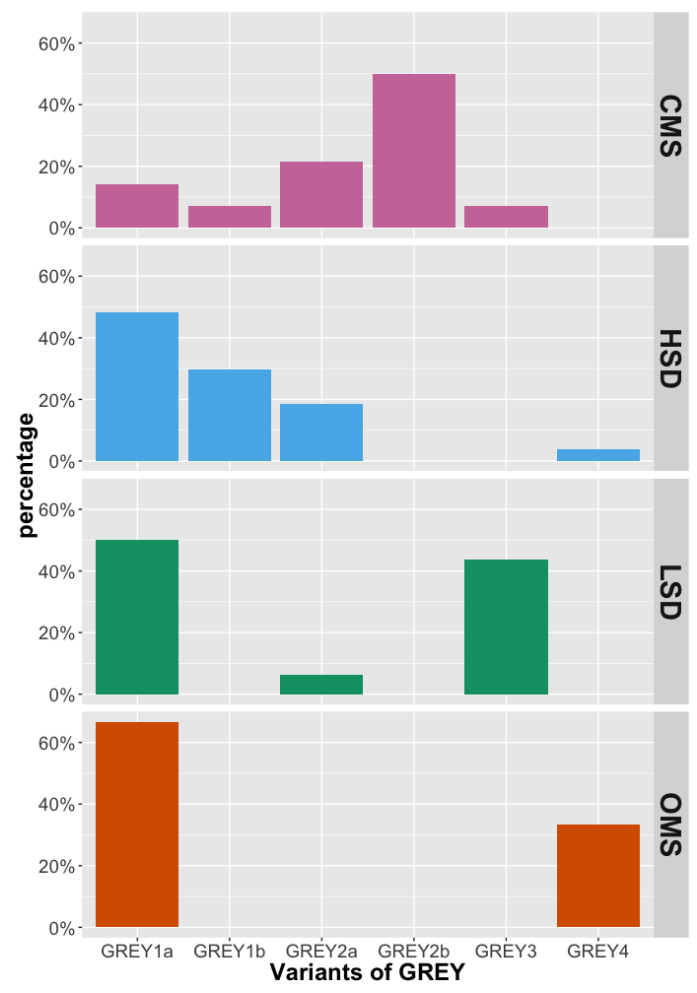

Figure 5.20: Percentage distribution by school: GREY (Fisher's Exact test, $\mathrm{p}<0.0001$ ) 


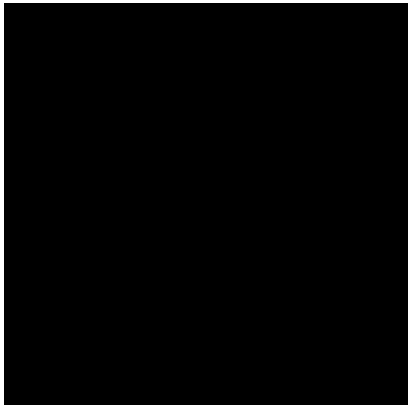

(a) GREY1a

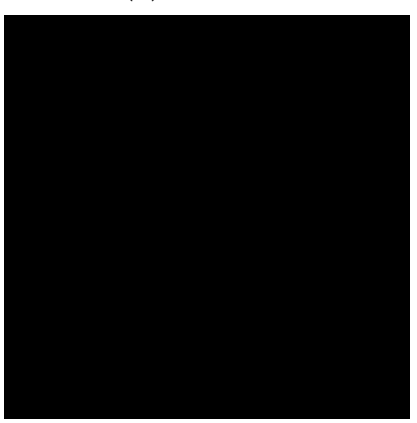

(c) GREY2a

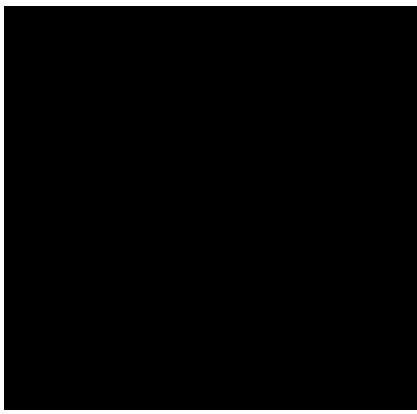

(e) GREY3

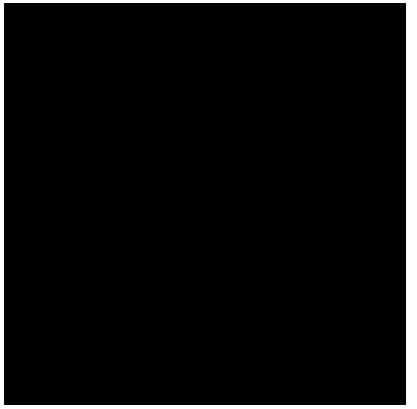

(b) GREY1b

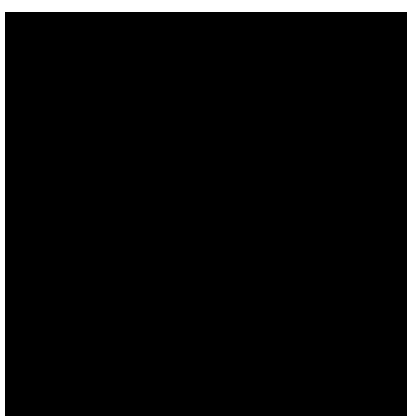

(d) GREY2b

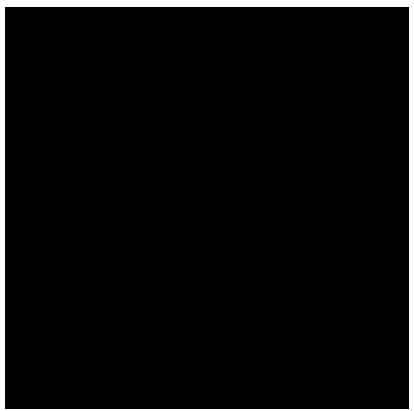

(f) GREY4

Figure 5.21: Major variants of GREY

\subsubsection{Kinship terms}

Kinship is the most stable category among the four semantic domains under investigation. The commonly used 'citation forms' are produced by a greater number of participants. Despite that, there are some innovative forms evolved from the 'citation form,' except for RELATIVES. The new 
forms FATHERb and MOTHERb are phonologically related subvariants of FATHERa and MOTHERa, in which the selected finger (thumb for FATHER and index finger for MOTHER) is placed at the side rather than at the centre of the mouth and chin area (see Figure 5.22). I remember when I first saw the new form MOTHERb, I could not associate it with the meaning mother and thought it was a new sign to learn (I was a novice signer at that time). I immediately asked for clarification from the young deaf signer who made the sign. She explained to me that she moved the index finger to the side of the mouth because it made it easier for her to mouth the word "mother." This may be one of the explanations for the creation of this innovative form and is possibly a side product of oralism.

For the 'citation form' of the four sibling terms, all of them are made by compounding a sign representing elder/younger and a sign representing male/female. For instance, ELDER^FEMALE is equal to ELDER-SISTER. The innovative forms drop the second part of the compound, i.e., MALE / FEMALE, and used just the first part to represent the four terms, i.e., ELDER for ELDER-BROTHER or ELDER-SISTER and YOUNGER for YOUNGER-BROTHER or YOUNGER-SISTER (see Appendix D, Figures 19 to 22 (e) for sign illustrations of both forms).

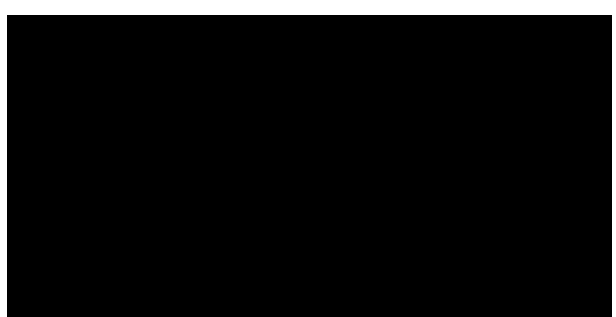

(a) FATHER

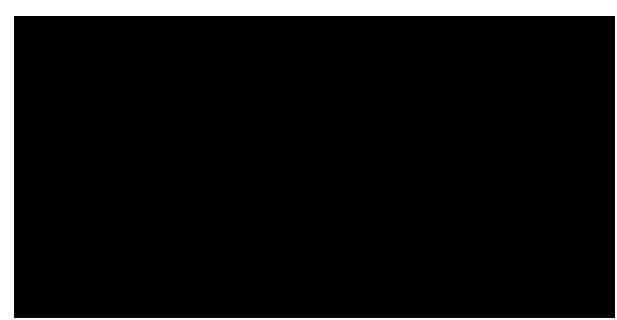

(b) MOTHER

Figure 5.22: Phonological subvariants of FATHER and MOTHER 
Figures 5.23 to 5.28 reveal that CMS and HSD are more likely to use the innovative forms for all these six kinship terms, while LSD and OMS tend to stick to the original forms. Indeed, none of the LSD and OMS participants used the innovative forms FATHERb and MOTHERb, and no OMS signers produced the new form ELDER-SISTER2. Nonetheless, only MOTHER and YOUNGER-SISTER demonstrate statistical significance in terms of difference between different schools (Fisher's Exact test, $\mathrm{p}<0.01$ in both cases). If the explanation about mouthing was the real origin of the new forms for FATHER and MOTHER, it could also explain why CMS and HSD signers are the main source of the new forms as these two schools were considered to be more strict than LSD and OMS in terms of speech training. In addition, it is observed that the new forms of the four sibling terms are commonly or even obligatorily accompanied with appropriate mouthing to distinguish the meaning of the sign. For instance, simultaneously articulating ELDER and mouthing the word 哥 (Cantonese for elder brother) to signify ELDER-BROTHER. Certainly, it is worth exploring the variation in the use of mouthing among different signers. Unfortunately, this is out of the scope of the current study. 


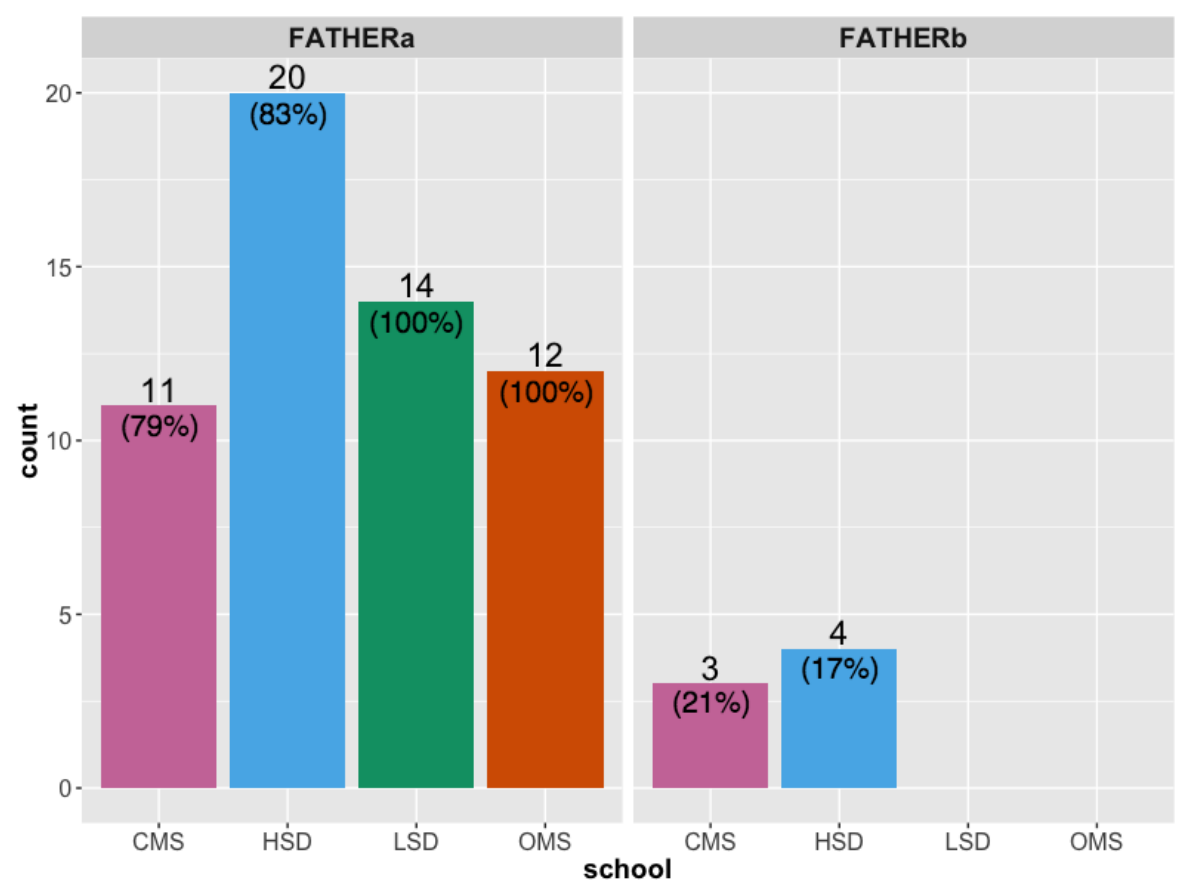

Figure 5.23: Frequency distribution by school: FATHER (Fisher's Exact test, $\mathrm{p}=0.1478$ )

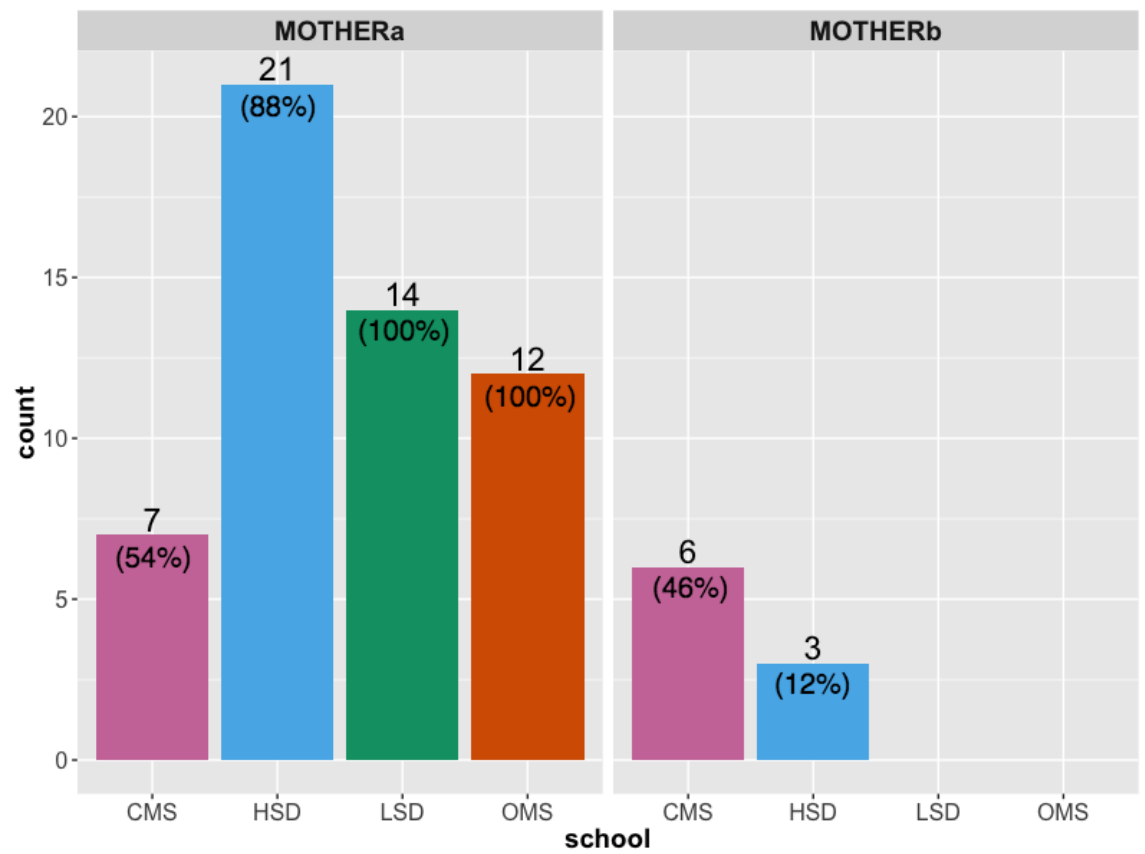

Figure 5.24: Frequency distribution by school: MOTHER (Fisher's Exact test, $\mathrm{p}<0.01$ ) 


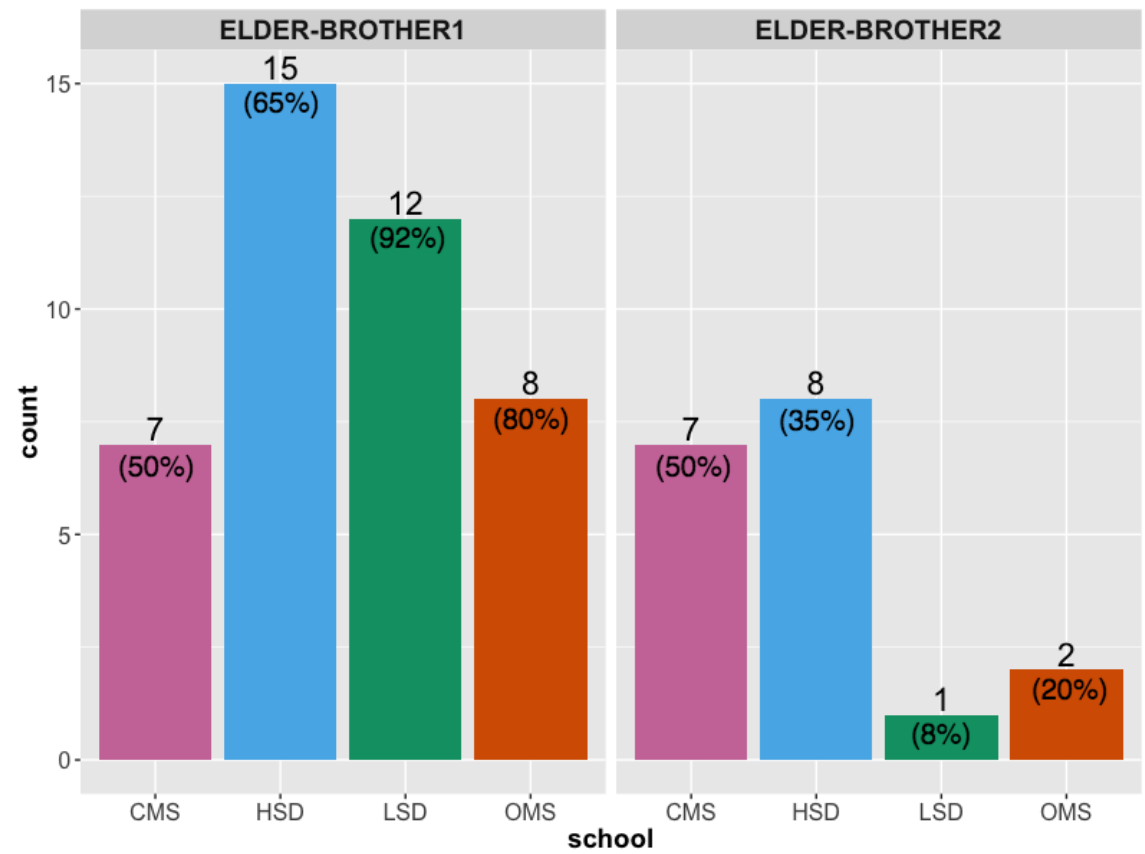

Figure 5.25: Frequency distribution by school: ELDER-BROTHER (Fisher's Exact test, $\mathrm{p}=0.0903)$

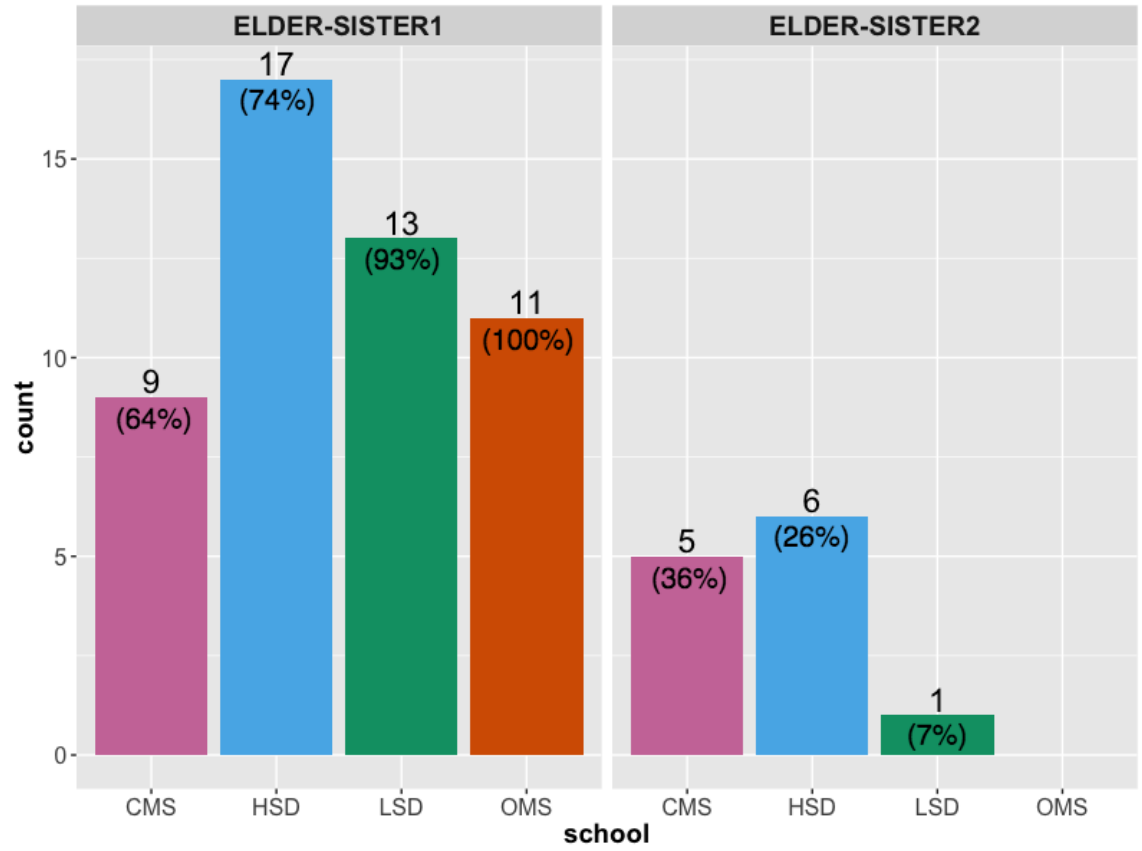

Figure 5.26: Frequency distribution by school: ELDER-SISTER (Fisher's Exact test, $\mathrm{p}=0.0677)$ 


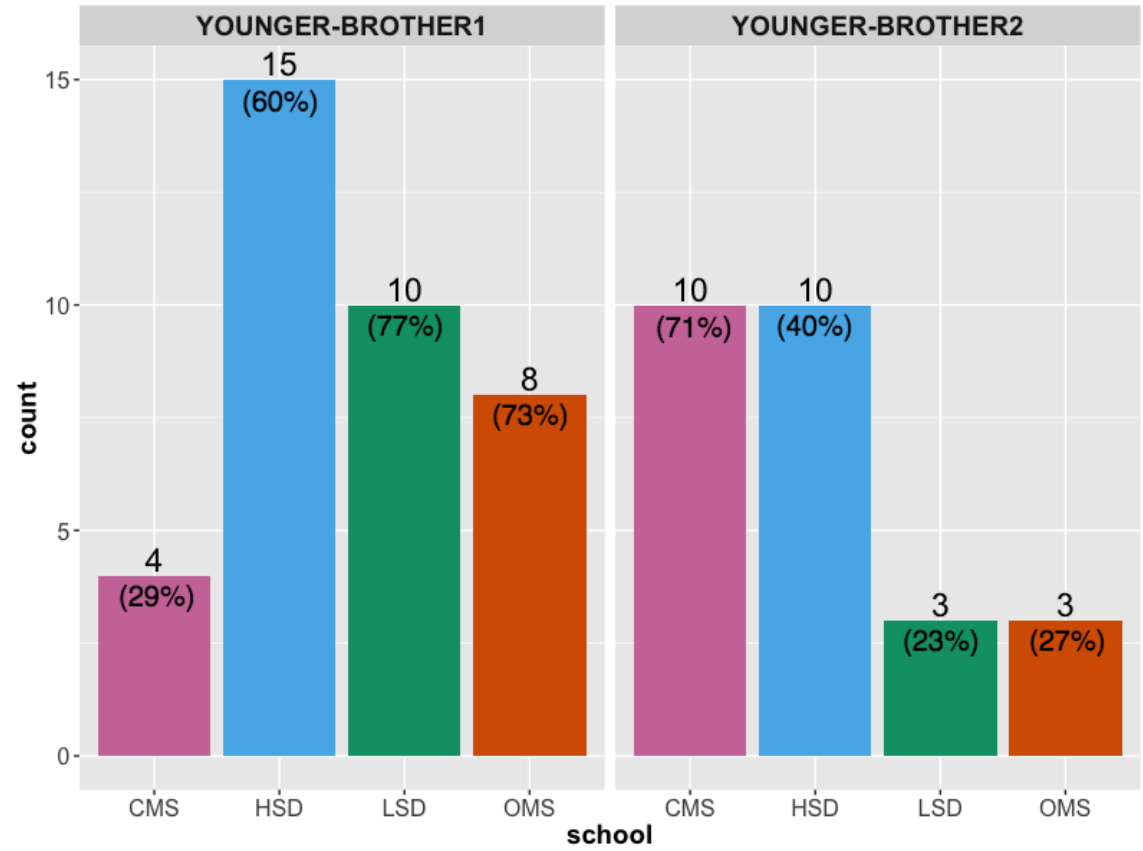

Figure 5.27: Frequency distribution by school: YOUNGER-BROTHER (Fisher's Exact test, $\mathrm{p}=0.0524)$

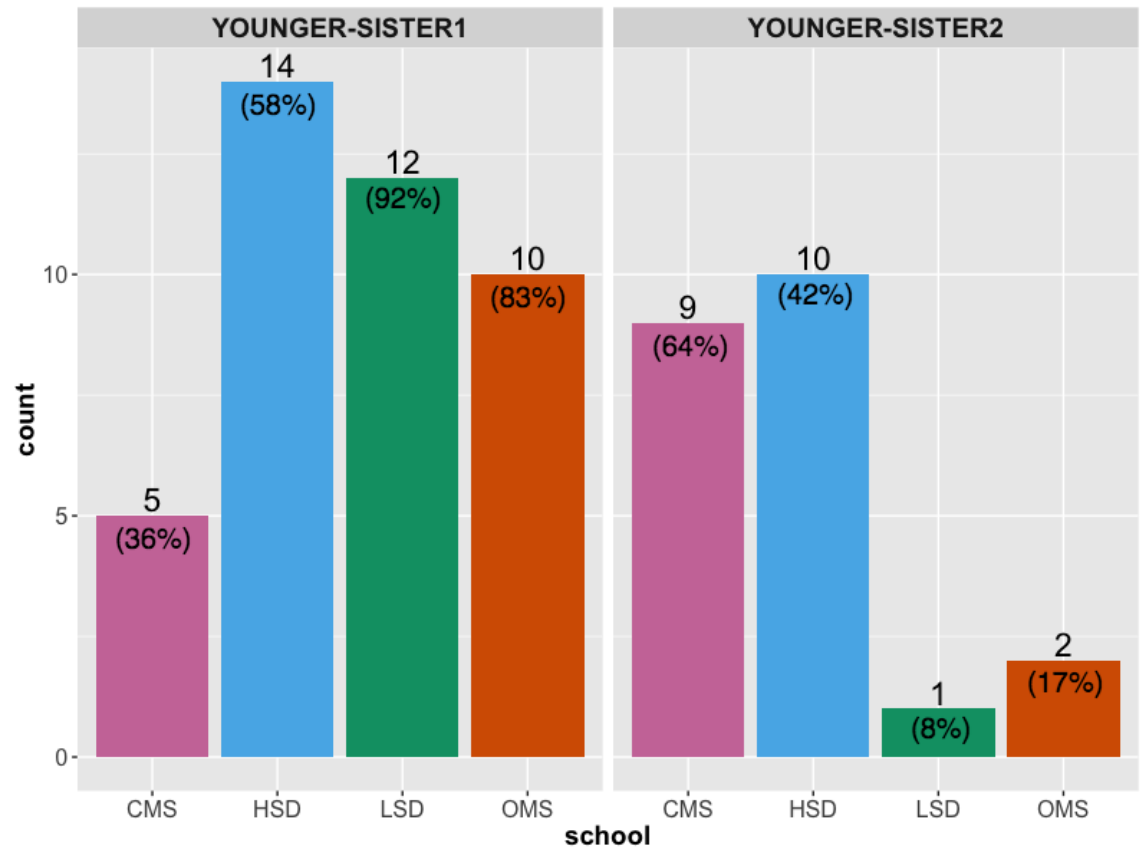

Figure 5.28: Frequency distribution by school: YOUNGER-SISTER (Fisher's Exact test, $\mathrm{p}<0.01)$ 


\subsubsection{Country/region names}

In the same way as the colour terms described earlier, different schools have different variant choices for some country/region names. Figures $5.29,5.30$, and 5.31 demonstrate such a pattern exemplified by AUSTRALIA, BEIJING, and INDIA respectively. Since many phonological subvariants are used by only a few participants, subvariants are grouped together and comparison is made between three major distinct variants in each sign. The three major variants of AUSTRALIA are illustrated in Table 5.8 on page 171 and Table 5.17 on page 182. Those of BEIJING and INDIA are shown in Figures 5.32 and 5.33. While CMS has no particular preference in AUSTRALIA and INDIA, they are the only one that favour BEIJING2 and they never use AUSTRALIA3, which is known to be an old sign (to be discussed in Section 5.4.2). Also, they are the only group that uses INDIA3 and they use it the most, suggesting this variant is originated from CMS. On the other hand, HSD tends to use the most frequent variant (i.e., AUSTRALIA1 and Beijing1) in both Australia and Beijing, but not in INDiA. They prefer INDIA2 slightly more. Moreover, OMS prefers the most frequent variant of AUSTRALIA and INDIA, but not BEIJING. They favour the compound form BEIJING3, which is indeed compounding BEIJING1 and BEIJING2. LSD seems to have no strong preference in AUSTRALIA and BEIJING, but a slight inclination for INDIA1 over INDIA2. 


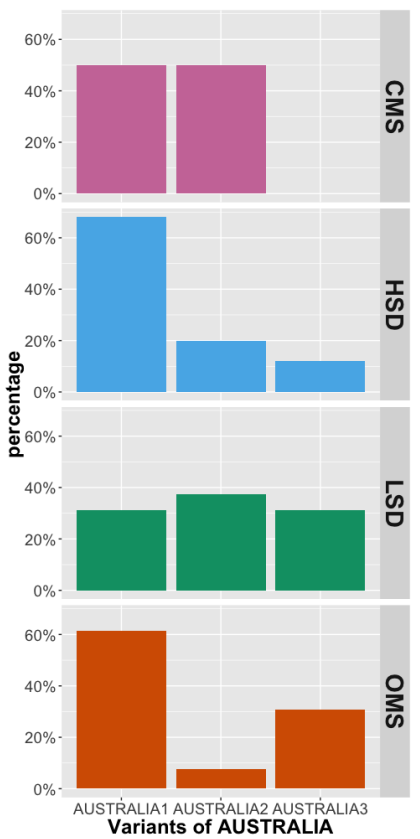

Figure 5.29: Percentage distribution by school: AUSTRALIA (Fisher's Exact test, $\mathrm{p}<0.05)$

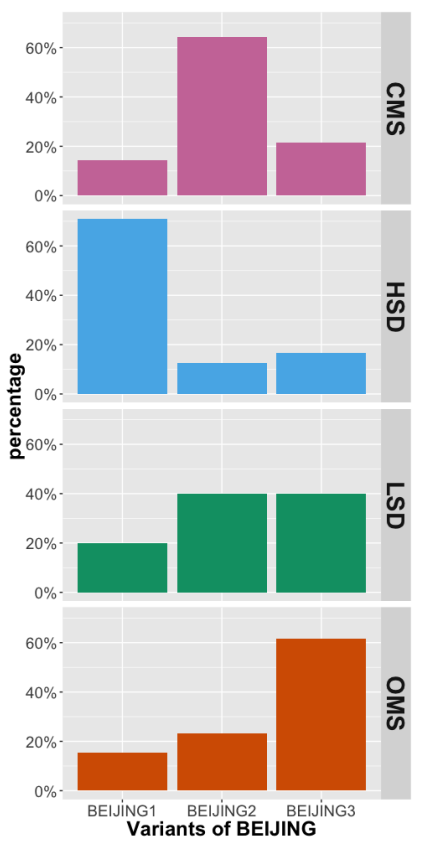

Figure 5.30: Percentage distribution by school: BEIJING (Fisher's Exact test, $\mathrm{p}<0.05)$

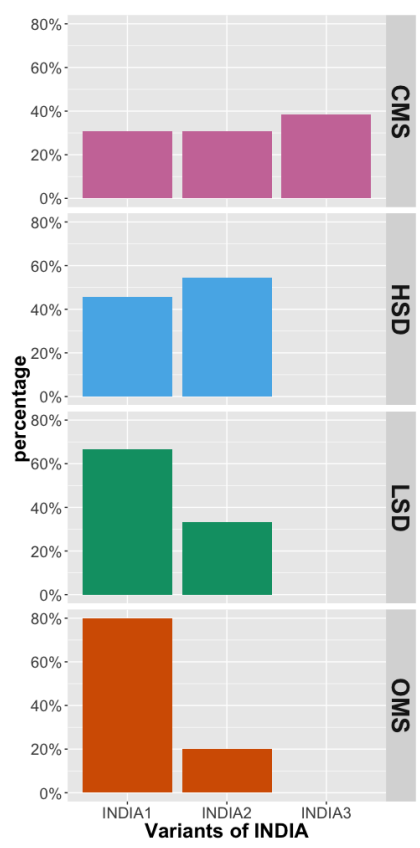

Figure 5.31: Percentage distribution by school: INDIA (Fisher's Exact test, $\mathrm{p}<0.05$ ) 


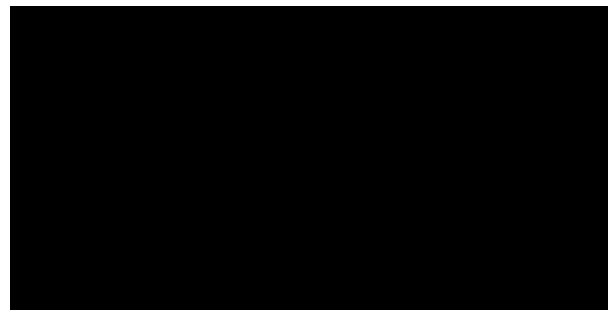

(a) BEIJING1a

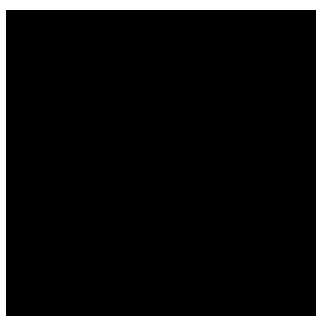

(c) BEIJING2a

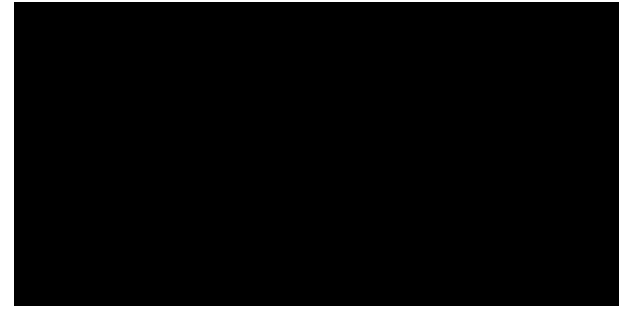

(b) BEIJING1b

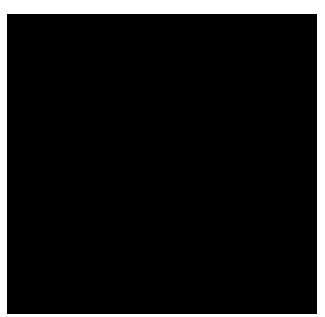

(d) BEIJING2b

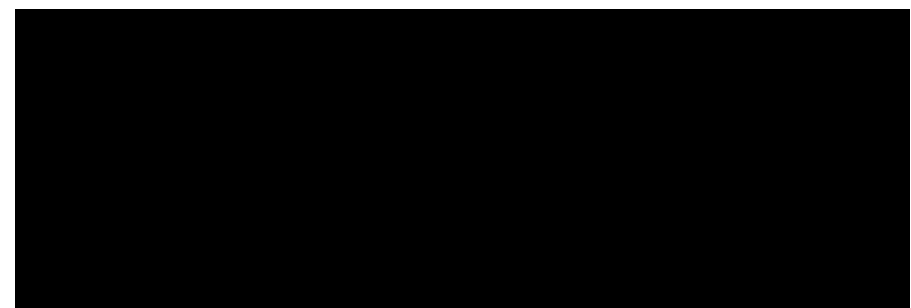

(e) BEIJING3a

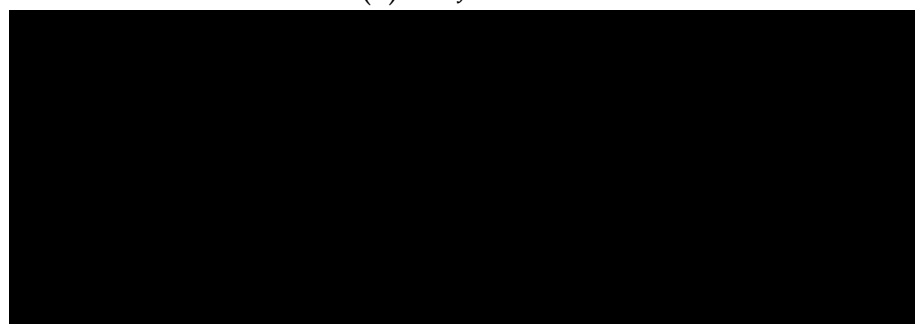

(f) BEIJING3b

Figure 5.32: Three major variants of BEIJING 


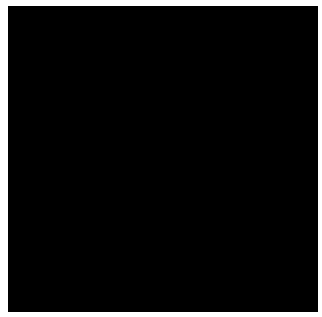

(a) INDIA1a

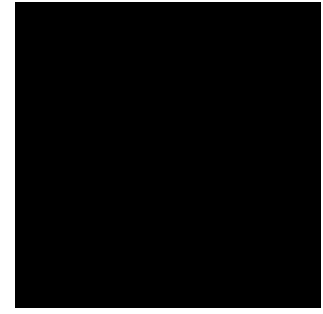

(b) INDIA2a

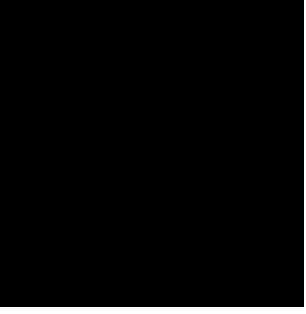

(c) INDIA2b

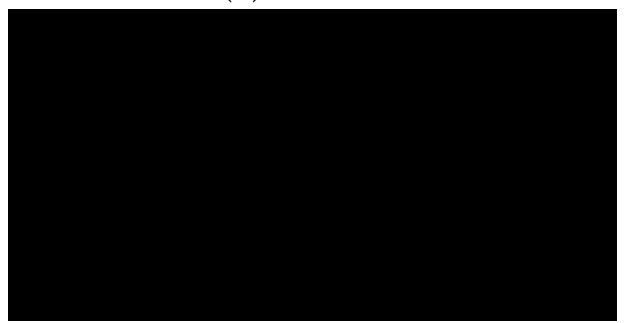

(d) INDIA3

Figure 5.33: Three major variants of INDIA

Further, some people from the deaf community thought the variant KOREA2 to be CMS related and VIETNAM2 to be associated with HSD. The data seems to support this observation. Figures 5.34 and 5.35 demonstrate that KOREA2 is largely used by CMS and HSD tends to use VIETNAM2. Both variants appear to find their way to other schools apart from LSD that only use VIETNAM1. This may be due to the fact that the two most frequent variants KOREA1 and VIETNAM1 are two handed signs while KOREA2 and VIETNAM2 are one handed signs (see Figures 5.36 and 5.37). All these variants are quite familiar to the Hong Kong deaf people. One may choose a simpler variant over a less simple one, such as one-handed over two-handed. Or one may opt for a variant that is articulated at a more common location over an uncommon location, for example, head over waist in the case of KOREA and face over above/upper head in VIETNAM. Having said that, both KOREA1 and VIETNAM1 have modified or simplified subvariants. For example, the location in KOREA1b has risen to the 
torso and the movement in KOREA1c is upward to a more central area rather than downward; VIETNAM1b is the one-handed version and the location in VIETNAM1c has dropped to the face area.

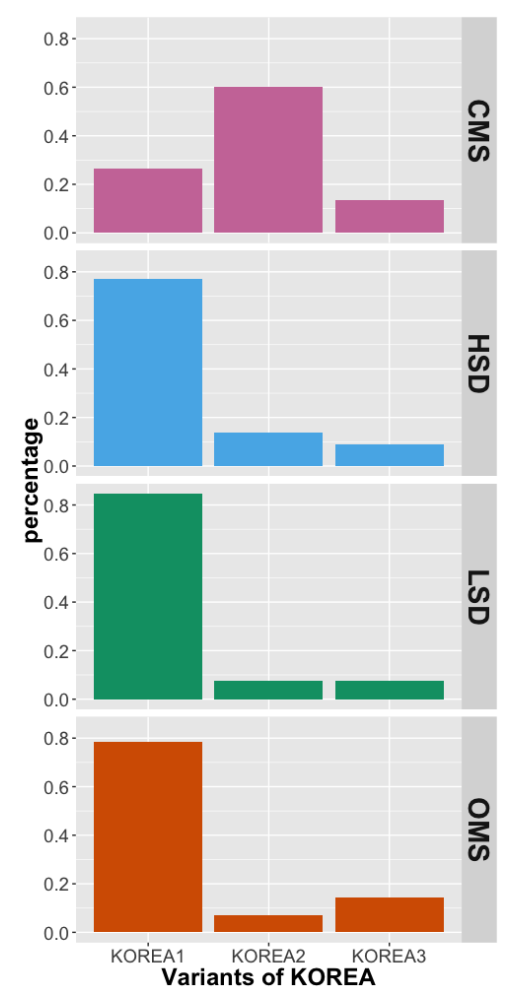

Figure 5.34: Percentage distribution by school: KOREA (CMS vs non-CMS, Fisher's Exact test, $\mathrm{p}<0.001)$

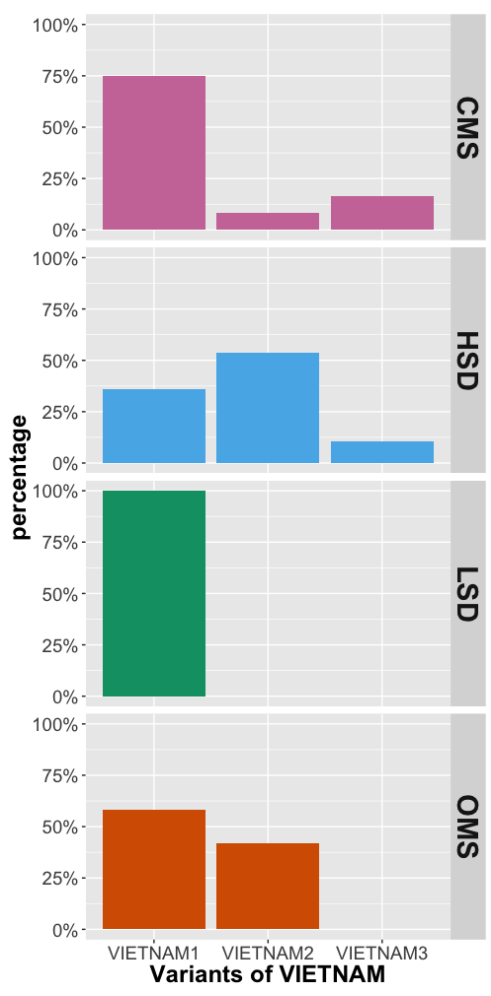

Figure 5.35: Percentage distribution by school: VIETNAM (HSD vs non-HSD, Fisher's Exact test, $\mathrm{p}<0.01$ ) 


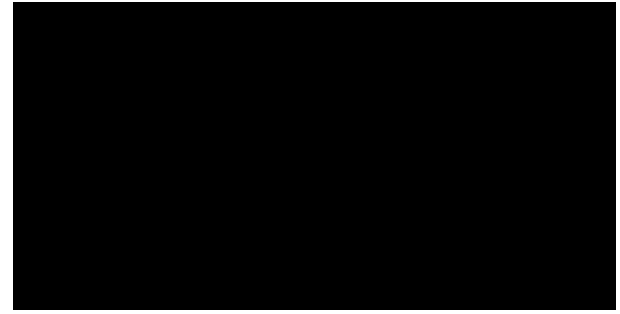

(a) KOREA1a

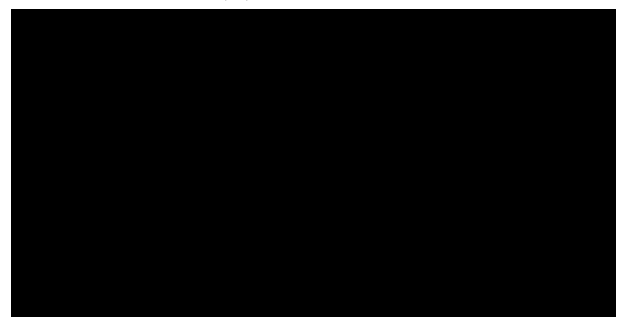

(c) KOREA1c

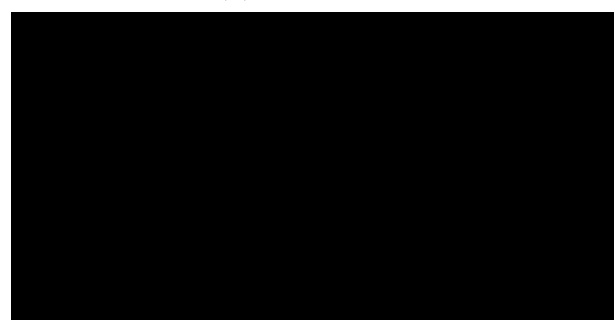

(e) KOREA1e

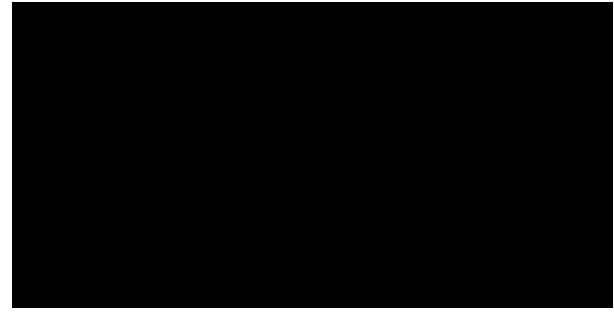

(b) KOREA1b

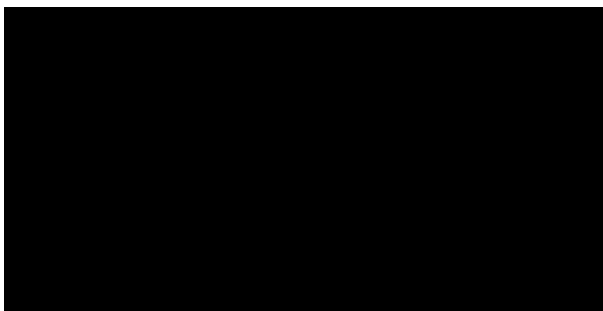

(d) KOREA1d

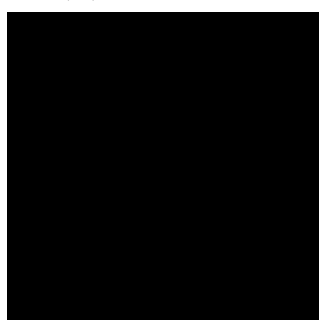

(f) KOREA2

Figure 5.36: Variants of KOREA

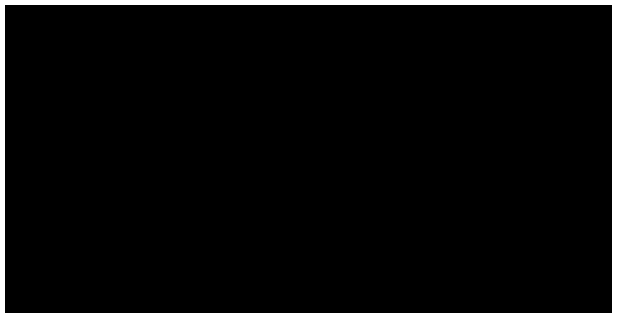

(a) VIETNAM1a

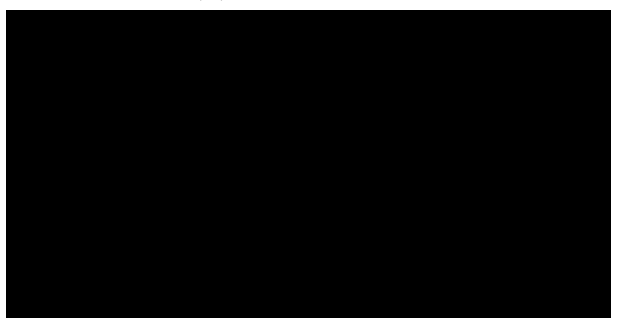

(c) VIETNAM1c

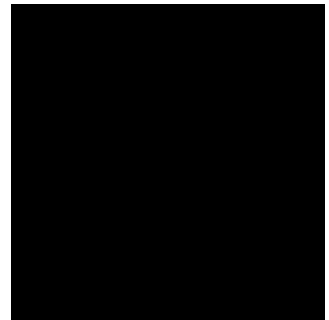

(b) VIETNAM1b

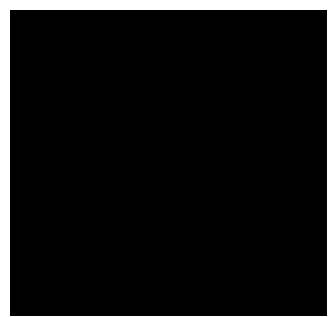

(d) VIETNAM2a

Figure 5.37: Variants of VIETNAM 


\subsubsection{Numbers}

In HKSL, numbers from one to ten have only one-handed forms and they are represented by specific handshapes. For example, holding the index finger in neutral signing space represents one and an open 5-handshape represents five. The numbers eleven and above are usually made by a combination of these handshapes to produce the corresponding number, such as a 2-handshape and a 5-handshape to mean twenty-five (though varying movements are observed). Numbers greater than ten can be signed either one-handed or two-handed. There are numbers that demonstrate schooldifferentiation in using one-handed versus two-handed variants, including ELEVEN, TWELVE, THIRTEEN, and HUNDRED. The issue of handedness in number signs is reviewed in Section 5.8 .

Within the ten numbers from ONE to TEN, three numbers that are known to have handshape variation were elicited in this study and they are THREE, SEVEN, and EIGHT. Both THREE and EIGHT show significant school association in variant choices. THREE has four variants and EIGHT has three variants, they involve differences in handshape and palm orientation (see Figures 5.38 and 5.39). In THREE, the most frequent variant THREEa is produced with the palm facing the signer and the last three fingers are selected and the second most frequent variant THREEb is made with the same handshape but with palm facing outwards. Likewise, the other two least frequent variants also with one palm facing inwards and the other facing outwards, but the selected fingers are the middle three, i.e., a different handshape from THREEa and THREEb. 


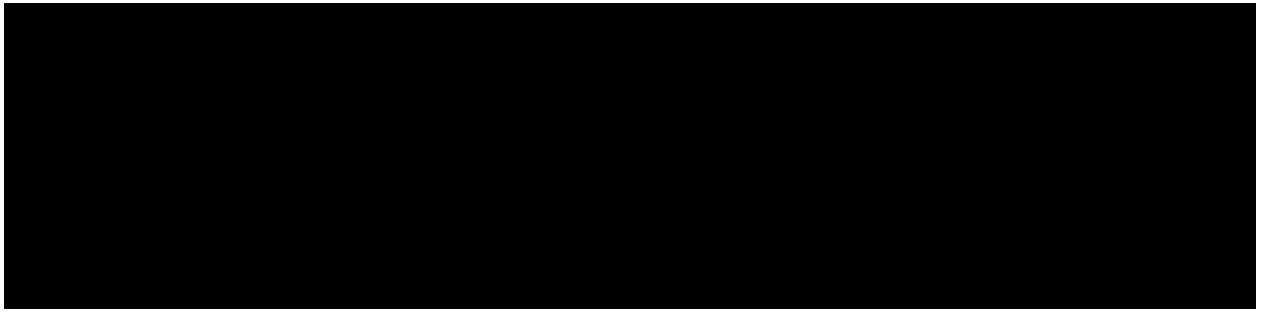
(a) THREEa
(b) THREEb
(c) THREEC
(d) THREEd

Figure 5.38: Variants of THREE

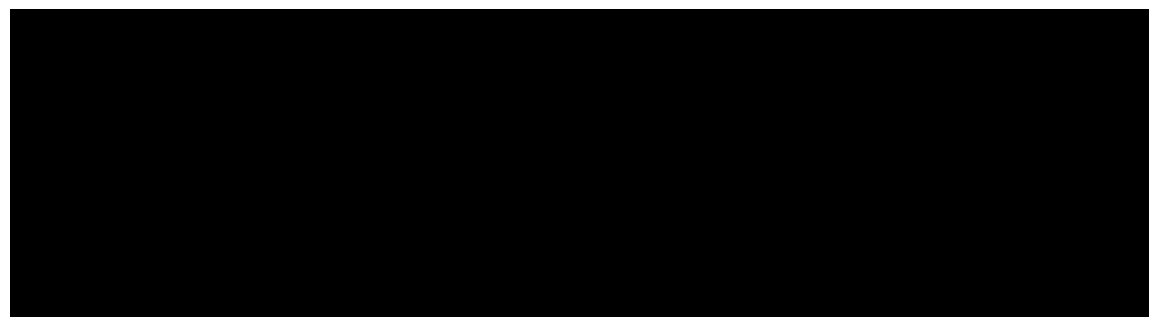

(a) EIGHTa

(b) EIGHTb

(c) EIGHTC

Figure 5.39: Variants of EIGHT

A Fisher's Exact test reveals that the different palm orientation in the variants of THREE is associated with school $(p<0.001)$, but the handshape differentiation is not $(p=0.1677)$. Figure 5.40 shows the frequency distribution of THREE by school. While HSD and OMS highly favour the palm-in variants, CMS and LSD seem to have no strong preference. On the other hand, variants of EIGHT appear to have strong association with school in terms of both handshape and palm orientation (both $\mathrm{p}<0.01$ ). EIGHTa is the most common variant with over $80 \%$ of the participants producing it and it is produced with a L-handshape with palm facing in. Both the two minor variants employ a different handshape which involve the first three fingers, one with palm facing in and the other facing out. As shown in Figure 5.41, all schools prefer the L-handshape form and the palm-in forms except CMS. Indeed, only CMS uses the variant EIGHTc and OMS only 
uses the variant EIGHTa.

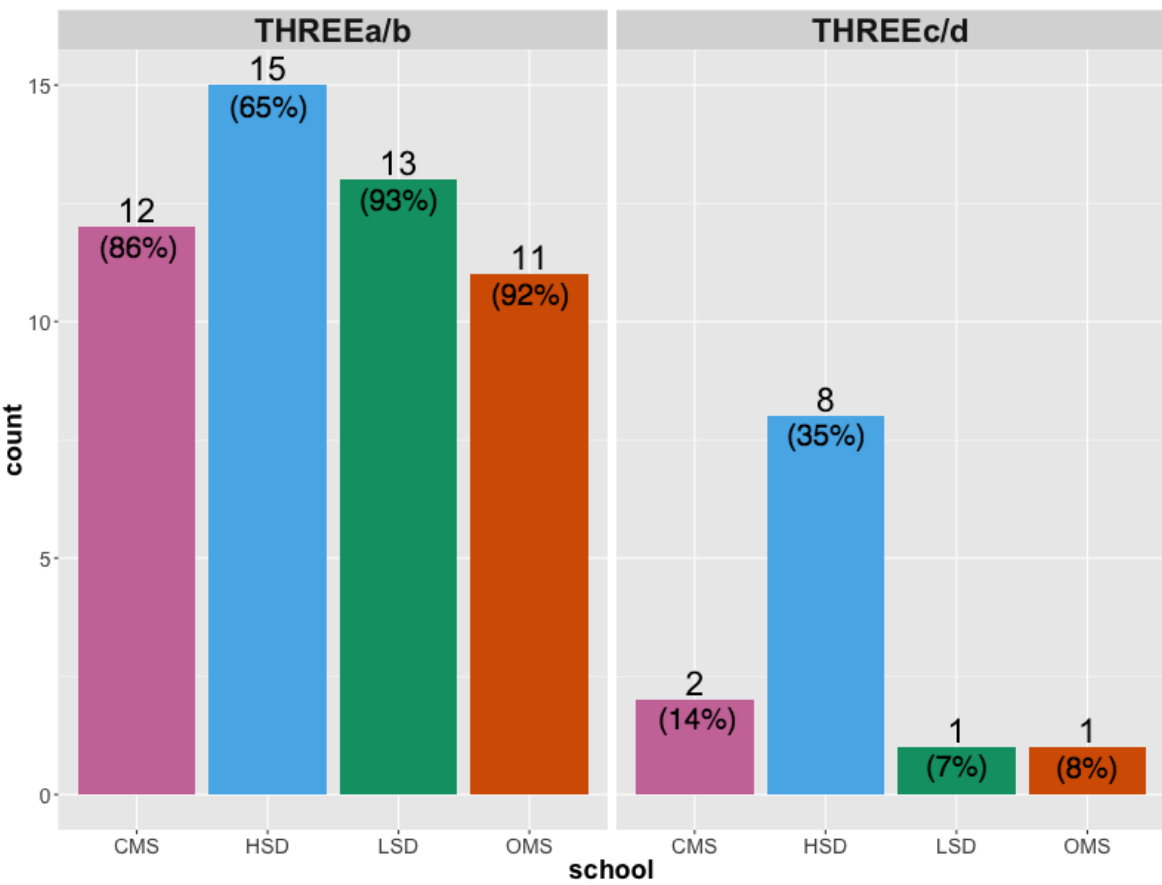

(a) THREEa/b vs THREEC/d (group by handshape)

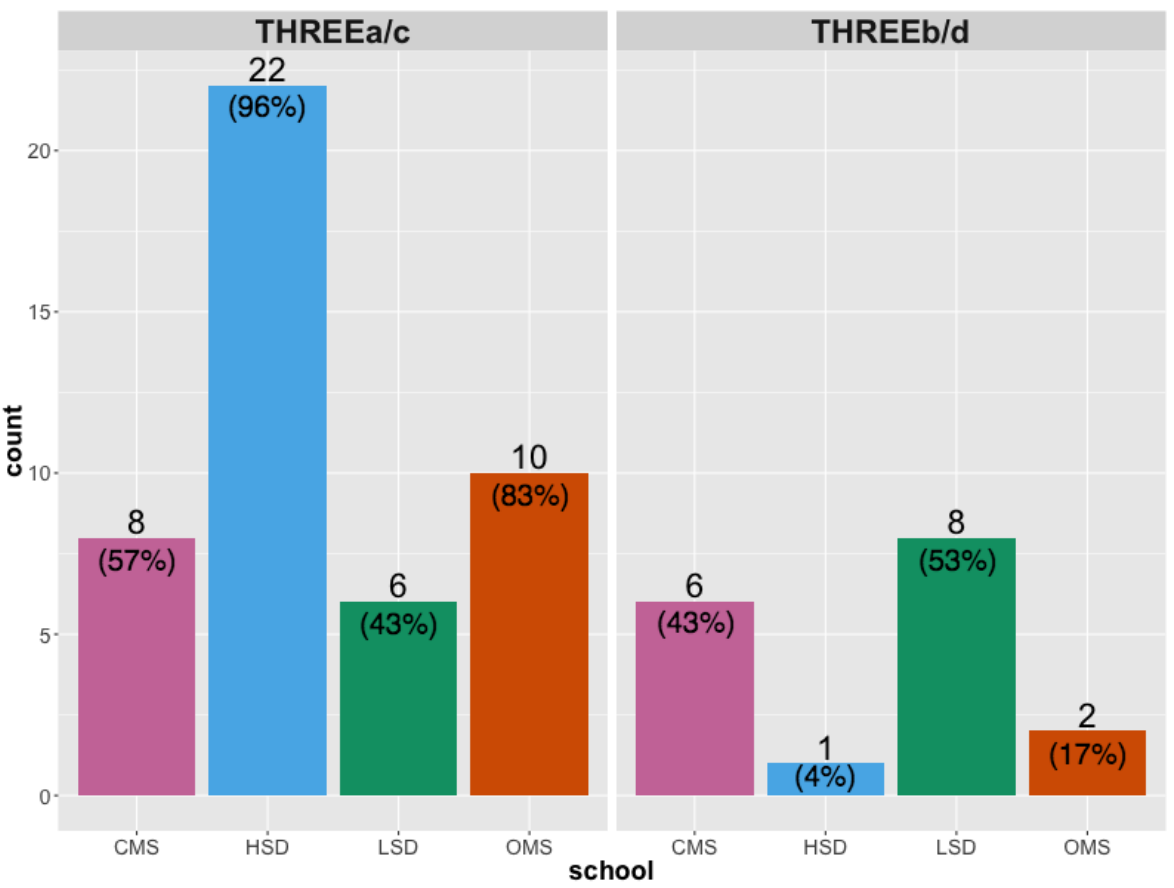

(b) THREEa/c vs THREEb/d (group by palm orientation)

Figure 5.40: Frequency distribution by school: THREE 


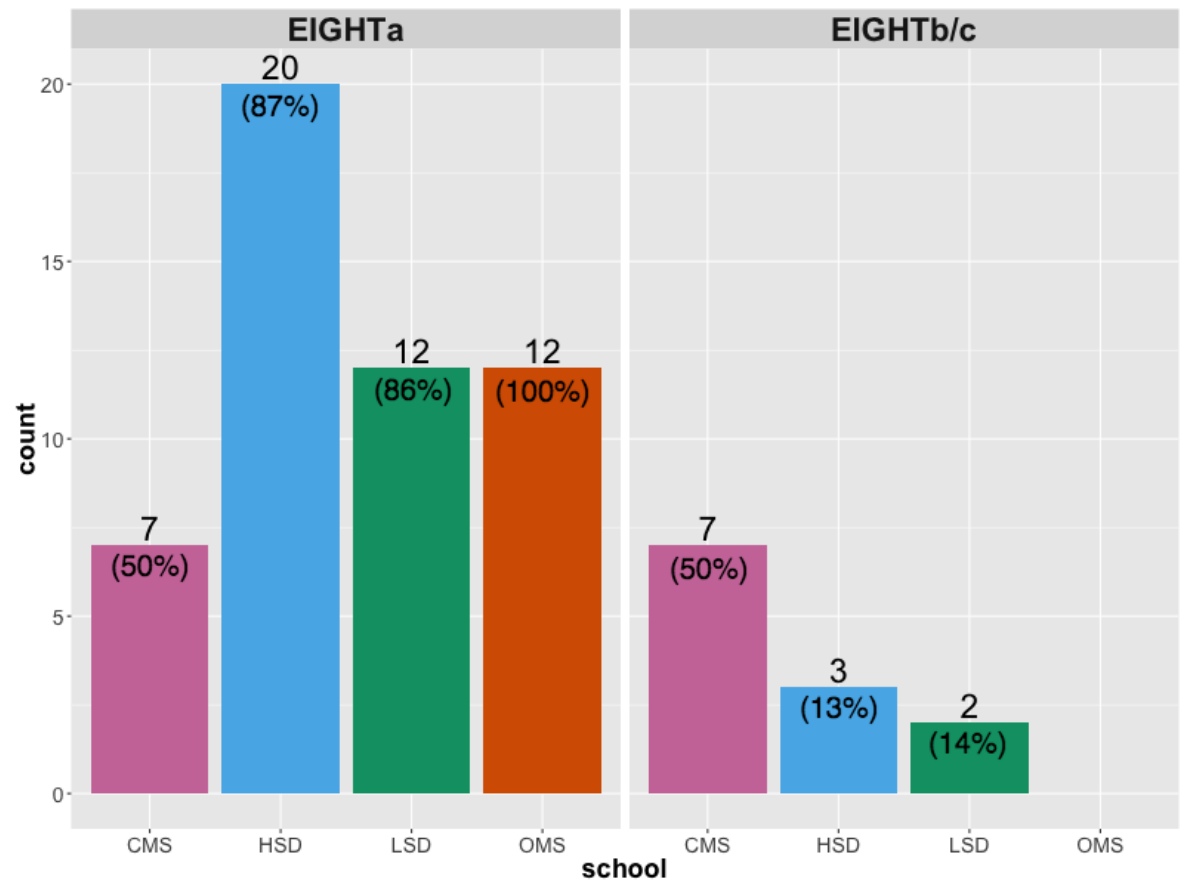

(a) EIGHTa vs EIGHTb/c (group by handshape)

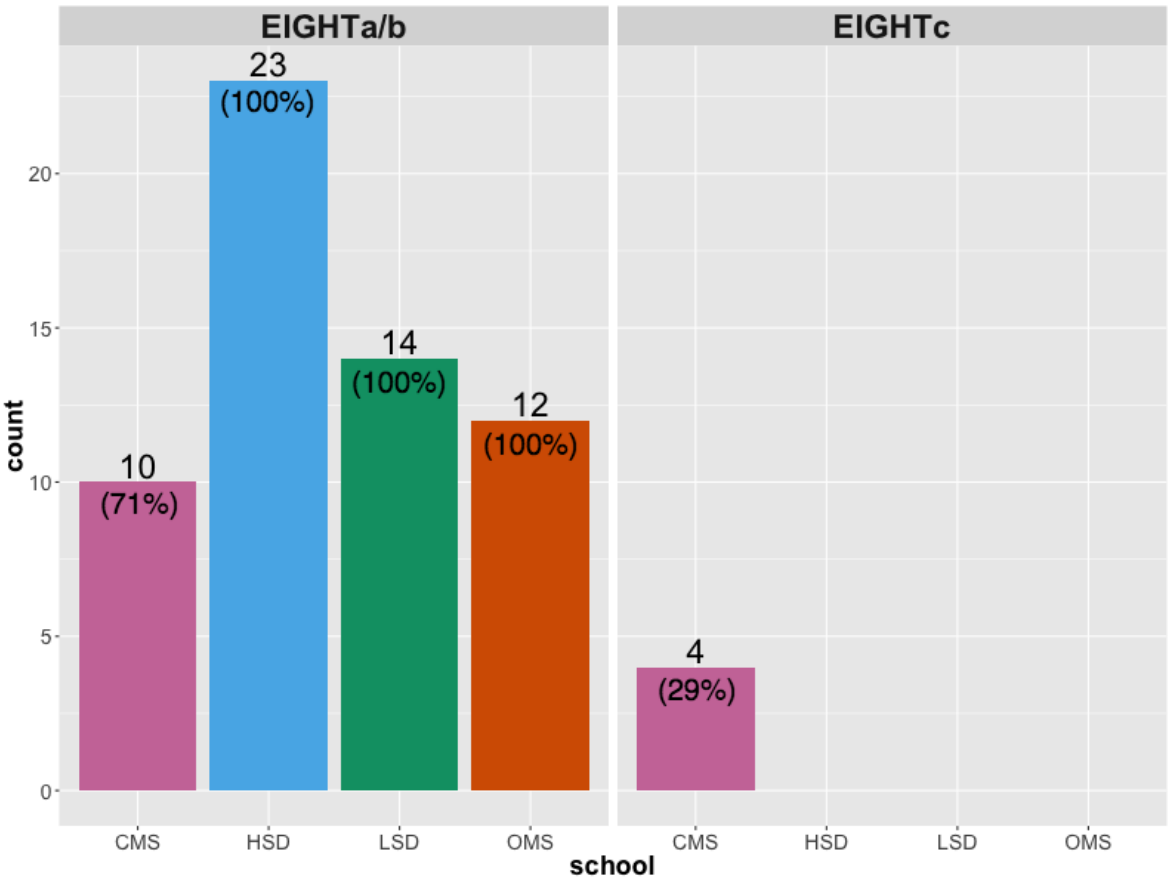

(b) EIGHTa/b vs EIGHTC (group by palm orientation)

Figure 5.41: Frequency distribution by school: EIGHT 
Another disparity of number use by CMS participants is seen in TWENTY and THIRTY. The major variants of the two number signs are TWENTY1a and THIRTY $1 \mathrm{a},{ }^{5}$ which are made by $75 \%$ and $65 \%$ of all participants respectively. Both TWENTY1a and THIRTY1a have the same movement, but different handshape. The former uses a 2-handshape and the latter uses a 3-handshape. Since THIRTY involves the use of a 3-handshape, both the last-three-finger and the middle-three-finger handshapes are being used. Like THREE, the last-three-finger handshape is more common and the most frequent variant THIRTY1a is made use of this handshape (see Figure 5.42). Another variant THIRTY $1 b$ is made of the middle-three-finger handshape, which differ from THIRTY1a in handshape only. Both variants are articulated by closing the selected fingers to make a fist and the two together comprise $78 \%$ of the total tokens. The variants TWENTY1c and THIRTY1c are produced by changing from a 2- or 3-handshape to a zero-handshape and they both have related variants. TWENTY1c and TWENTY1d differ in palm orientation only; both THIRTY1C and THIRTY1d use the last-threefinger handshape but one with palm-in and the other palm-out, and THIRTY1e use the middle-three-finger handshape with palm-in (see Figure 5.42).

\footnotetext{
${ }^{5}$ Unlike the coding of other lexical items, number variants are coded with 1 or 2 to signal one-handed and two-handed variants, then followed by a letter.
} 


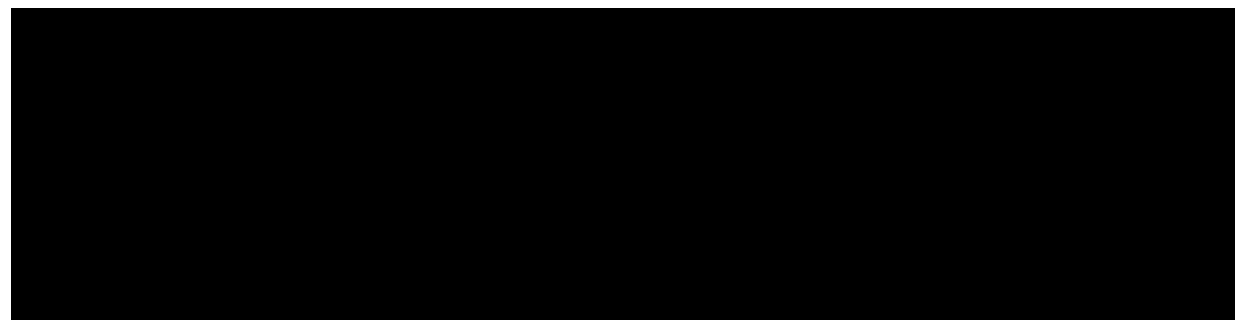

(a) THIRTY1a

(b) THIRTY1b

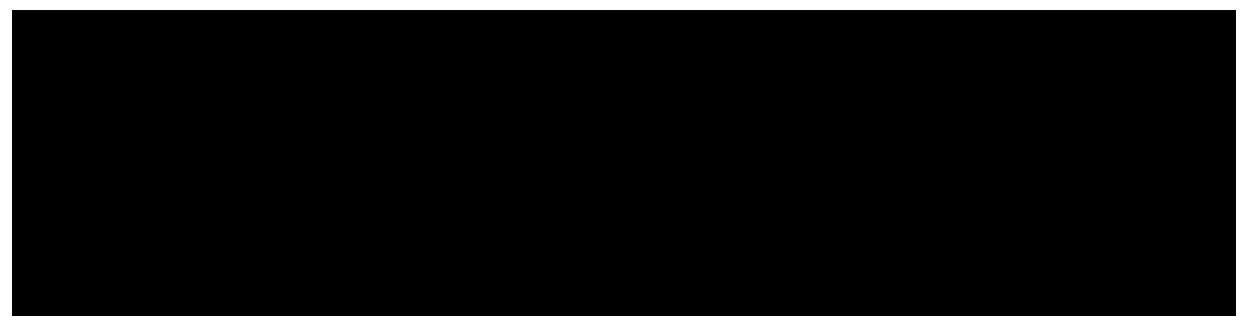

(c) THIRTY1C

(d) THIRTY1d

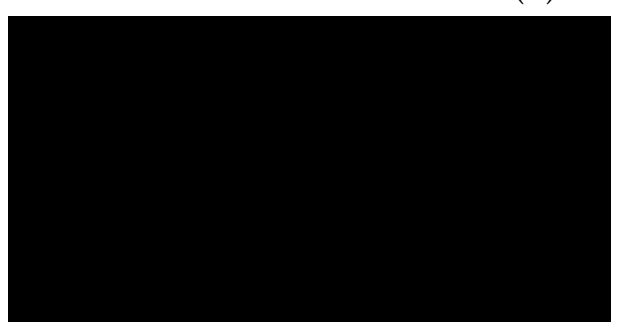

(e) THIRTY1e

Figure 5.42: Variants of THIRTY

Considering the movement difference is more prominent when comparing TWENTY1a with TWENTY1c/d and THIRTY1a/b with THIRTY1c/d/e, the variants are grouped accordingly and Figure 5.43 illustrates the unique pattern of CMS. While all other schools tend to use the most frequent forms in TWENTY and THIRTY, i.e., TWENTY1a and THIRTY1a/b, CMS seems to have no clear preference in the two number signs. 


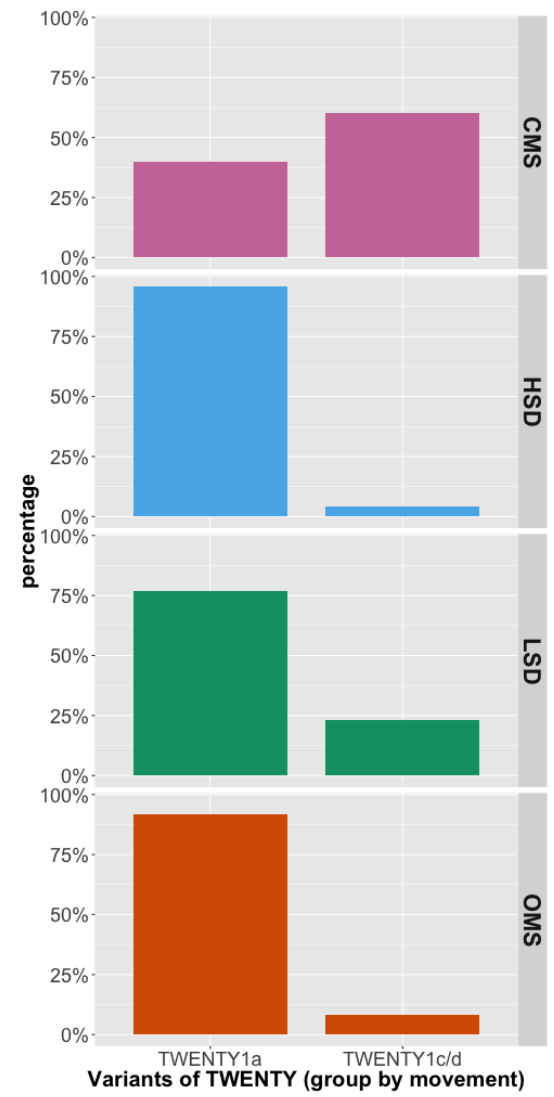

(a) TWENTY1a vs TWENTY1c/d (Fisher's Exact test, $\mathrm{p}<0.01$ )

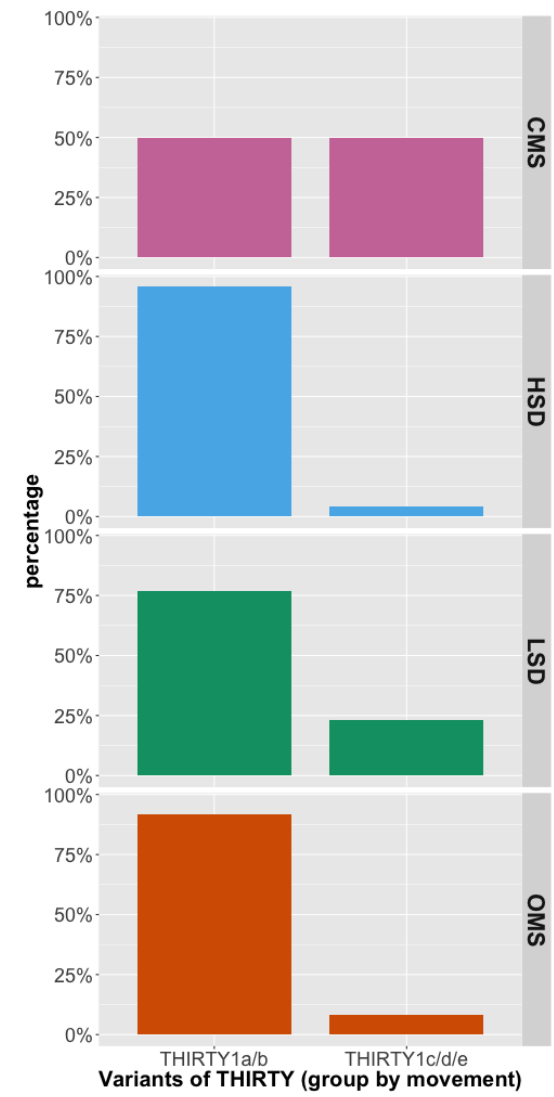

(b) THIRTY1a/b vs THIRTY1c/d/e (Fisher's Exact test, $\mathrm{p}<0.01$ )

Figure 5.43: Percentage distribution by school: TWENTY and THIRTY

Apart from the signs TWENTY and THIRTY, significant movement preference by schools also appears in FIFTEEN and some numbers in the twenties. In FIFTEEN, all variants are made of the combination of a 1-handshape and a 5-handshape, except one produced by an older signer. The most common variant FIFTEEN1a is produced by simply changing from a 1handshape to a 5-handshape without moving the hand and the location of articulation remains, while the second most popular variant FIFTEEN1b is also made by changing the handshape from 1 to 5 , but there is a slight hand movement in the transition of handshape change (see Figure 5.44). 
Figure 5.45 presents the frequency distribution of the two movement patterns across schools $(\mathrm{p}<0.001){ }^{6}$ We can see from Figure 5.45 that HSD highly prefers FIFTEEN1a and LSD tends to use FIFTEEN1b/c more.

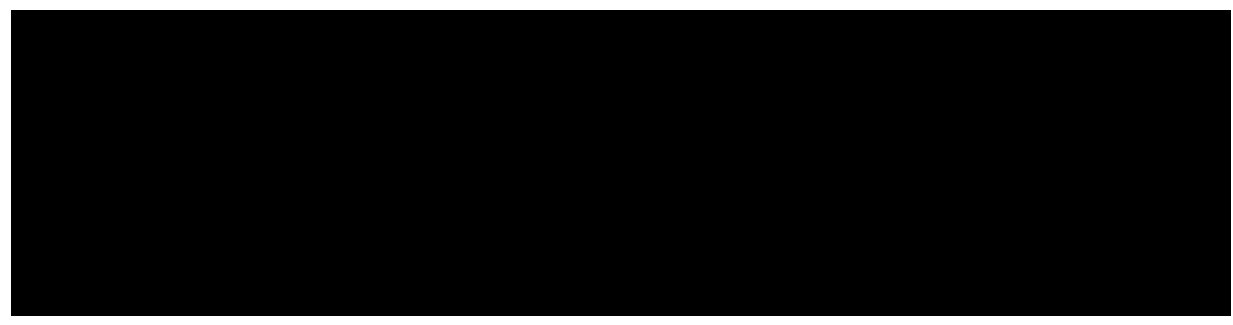

(a) FIFTEEN1a

(b) FIFTEEN1b

Figure 5.44: Major variants of FIFTEEN

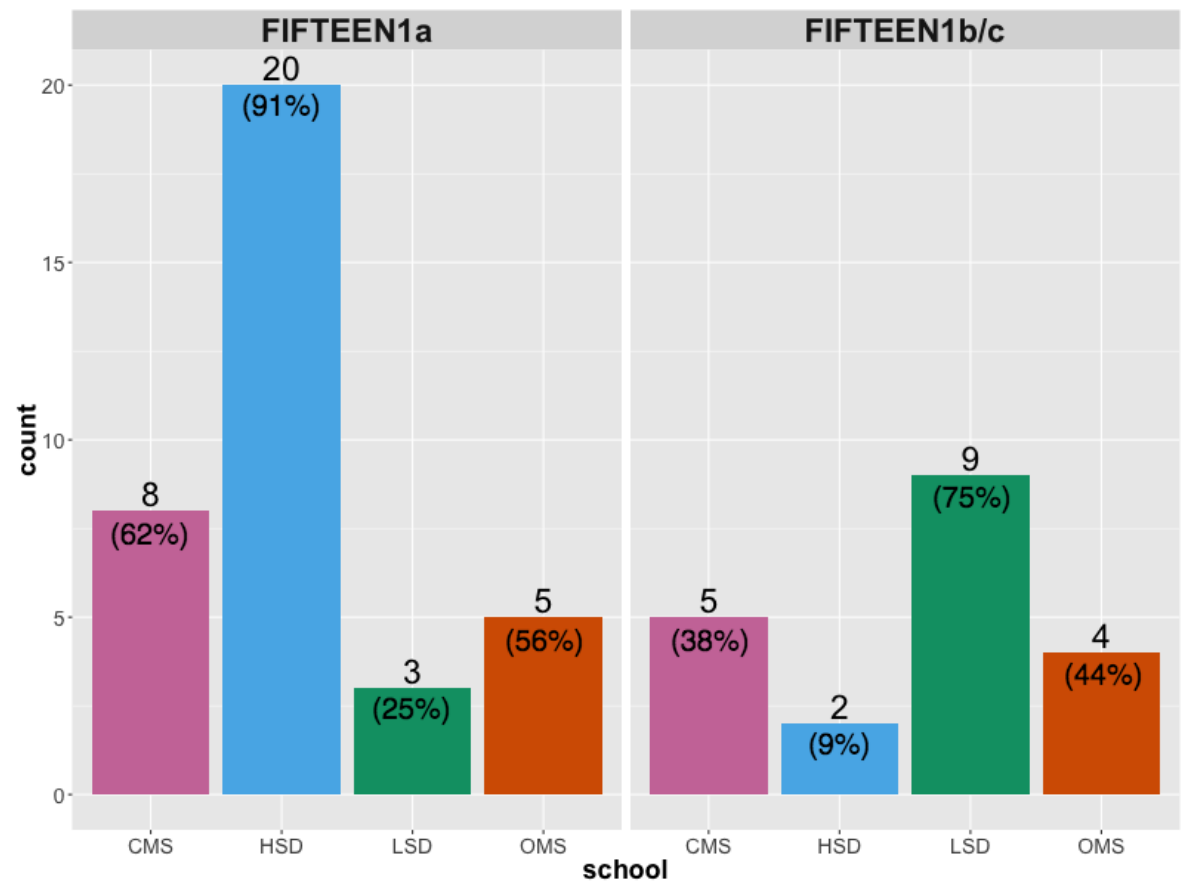

Figure 5.45: Frequency distribution by school: FIFTEEN (Fisher's Exact test, $\mathrm{p}<0.001$ )

${ }^{6} \mathrm{~A}$ minor variant (FIFTEEN1c) with the same movement as FIFTEEN1b but having palm-in is grouped with FIFTEEN1b. 
The two different movements found in FIFTEEN are also observed in most of the twenties. In addition, there are two other types of movement in all the twenties. One is a closing of a 2-handshape (i.e., TWENTY1a), then extending the selected fingers to represent the corresponding number. That is, the form is like TWENTY1a plus a number (Figure 5.46d). Such a variant differs in popularity among the six twenties signs. In TWENTYONE, it is the most frequent variant (36\%); whereas in TWENTY-TWO, only one participant produced it. Another type of movement is wrist-twist (Figure 5.46c). Like the move-to-side variant, this wrist-twist movement happened during the transition of handshape change. This movement is mostly favoured in TWENTY-TWO where no handshape change is required $(68 \%)$. Such a form is also the most frequent variant in TWENTY-SEVEN $(38 \%)$. This may due to the finger closed feature of SEVENa (Figure 5.47), the most common form for SEVEN.

The four different movements are very often the major variants in the twenties, though in varying proportions. Statistical significance is found in TWENTY-ONE, TWENTY-TWO, TWENTY-FIVE, and TWENTY-SEVEN regarding school preference (all $\mathrm{p}<0.05$ ). Figures 5.48 to 5.51 illustrate the variant choices across schools. For reader convenience, the variants are coded as NUMBERhandshape, NUMBERmoveSide, TWENTYplus and NUMBERtwist referring respectively to the variants that simply change the handshape, the variant that adds movement during transition of handshape change, the one with TWENTY1a plus a number, and the form with wrist-twist (see Figure 5.46 for the exemplification of the various forms in TWENTY-ONE). In addition, variants of the same movement but with a different handshape or palm orientation are grouped together. 


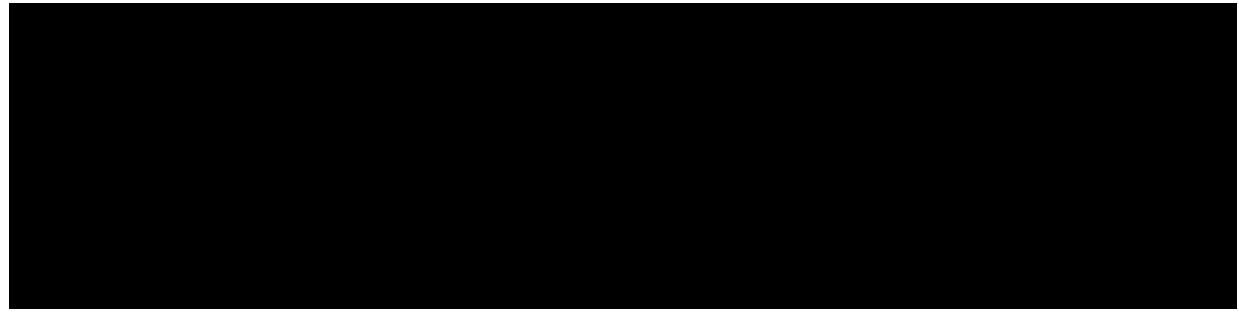

(a) TWENTY-ONEhandshape

(b) TWENTY-ONEmoveSide

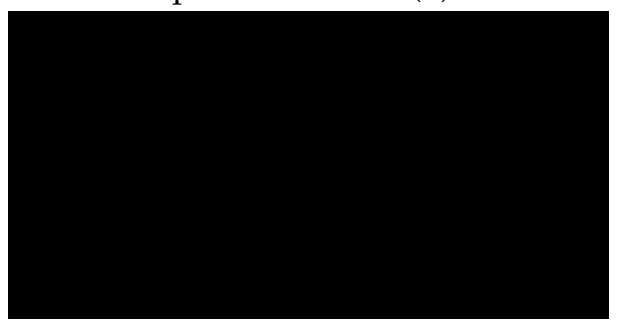

(c) TWENTY-ONEtwist

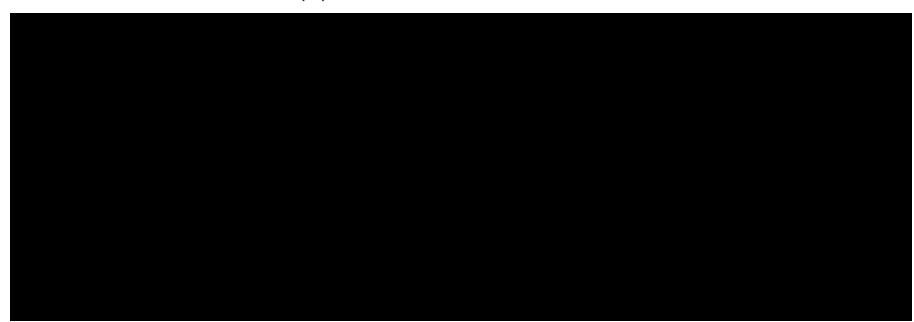

(d) TWENTYplus

Figure 5.46: Variants of TWENTY-ONE

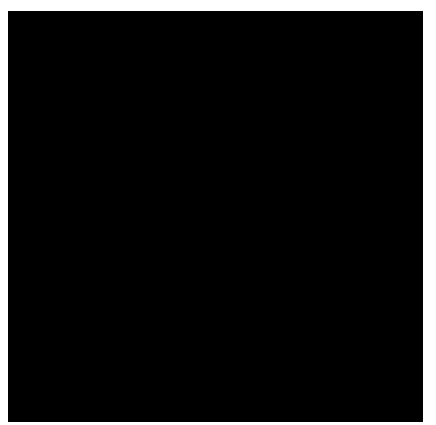

Figure 5.47: SEVENa 


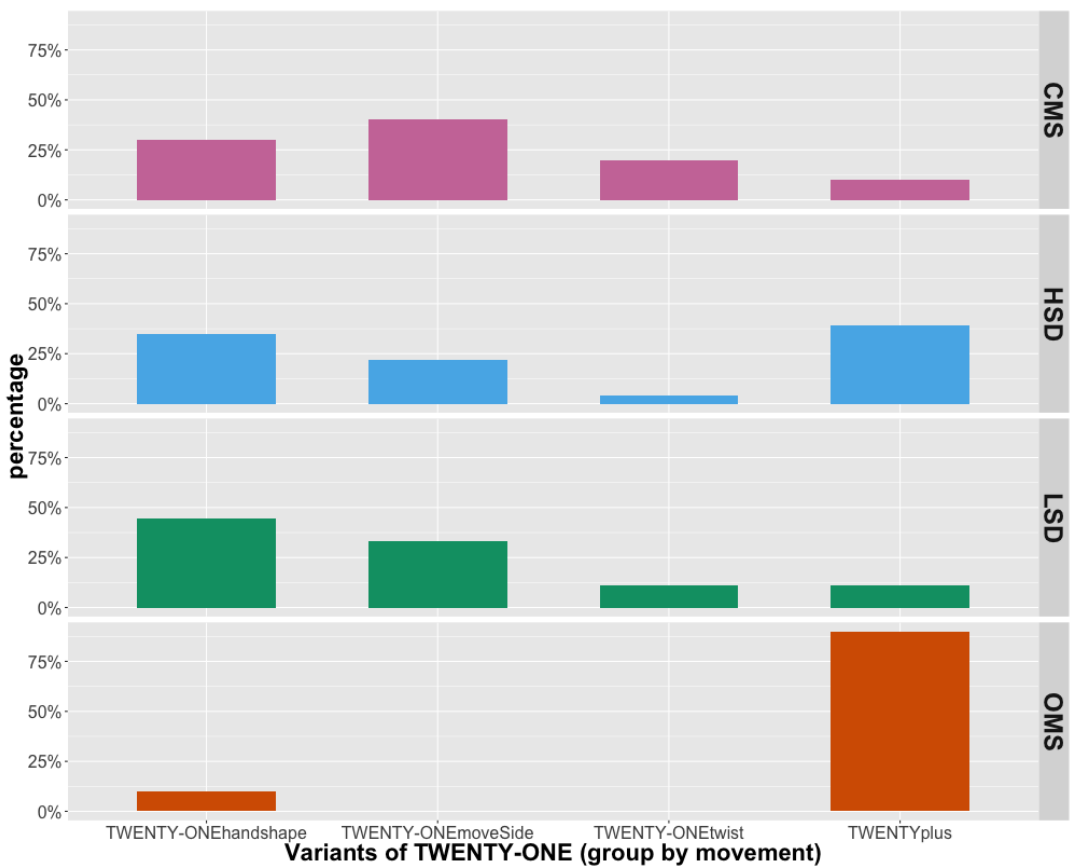

Figure 5.48: Percentage distribution by school: TWENTY-ONE (Fisher's Exact test, $\mathrm{p}<0.05$ )

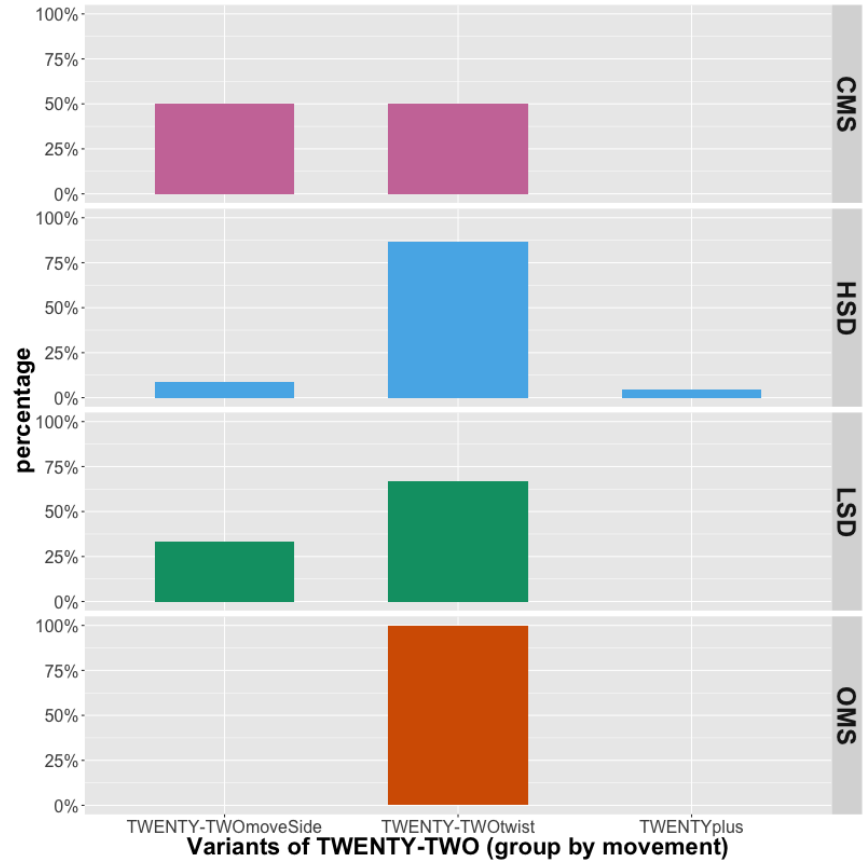

Figure 5.49: Percentage distribution by school: TWENTY-TWO (Fisher's Exact test, $\mathrm{p}<0.05$ ) 


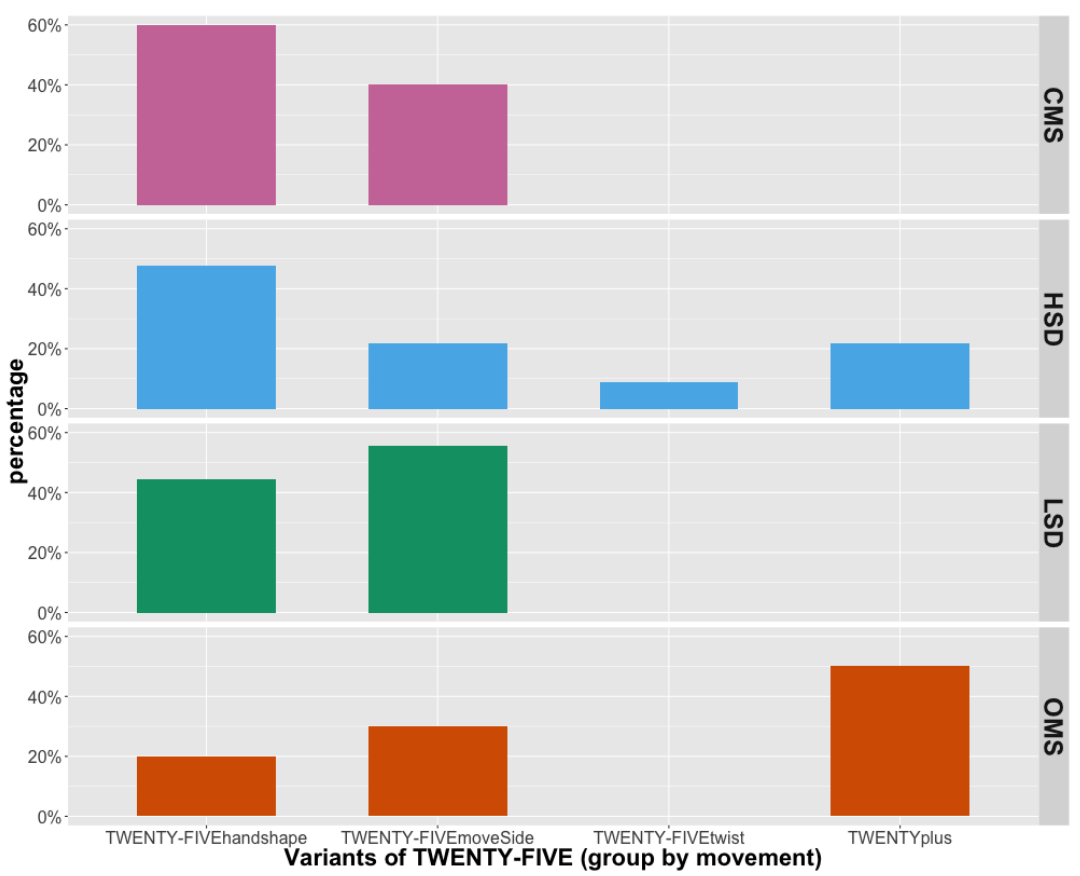

Figure 5.50: Percentage distribution by school: TWENTY-FIVE (OMS vs non-OMS, Fisher's Exact test, $\mathrm{p}<0.05)$

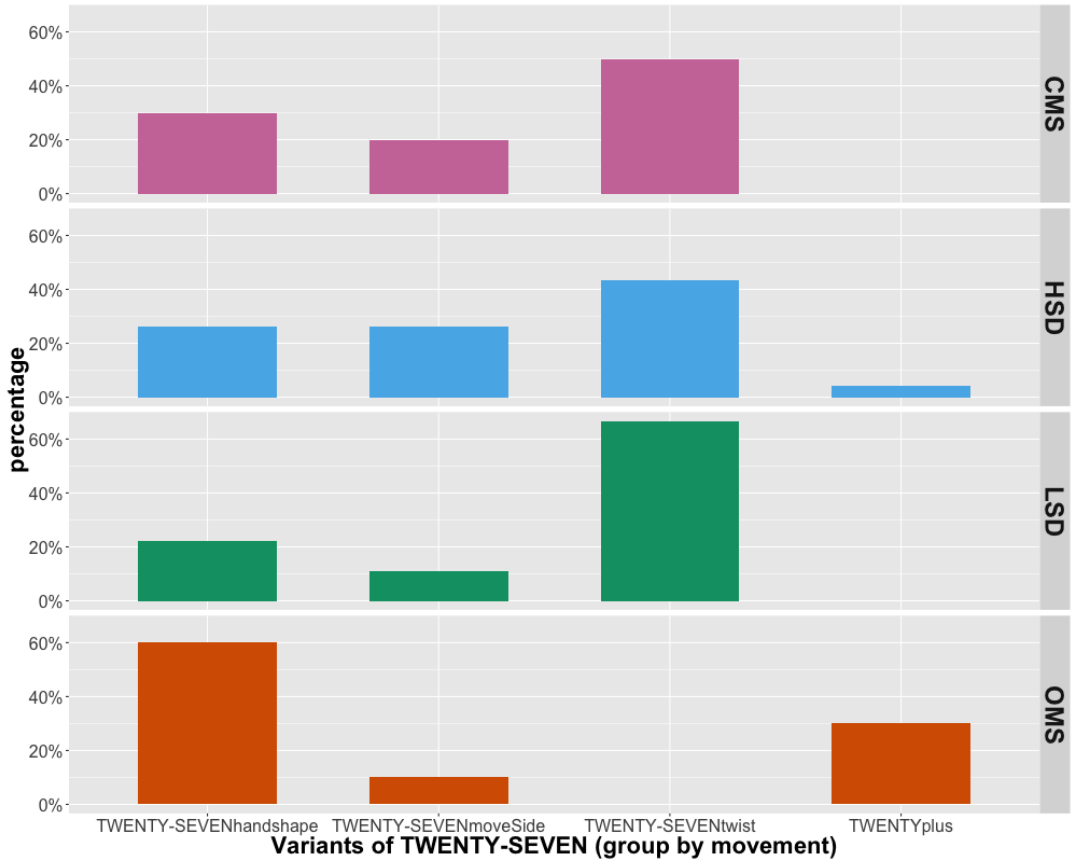

Figure 5.51: Percentage distribution by school: TWENTY-SEVEN (Fisher's Exact test, $\mathrm{p}<0.05)$ 
We can see that OMS seems to favor the TWENTYplus variants more than other schools, especially in TWENTY-ONE (Figure 5.48) and TWENTYFIVE (Figure 5.50). The handshape change variants appear to be favoured by people who attended CMS, HSD, and LSD, but the wrist-twist variants are used most with TWENTY-TWO (Figure 5.49) and TWENTY-SEVEN (Figure 5.51). Yet, while the wrist-twist variant gets the highest numbers in HSD, LSD, and OMS for TWENTY-TWO, only half of the CMS signers chose it and the other half prefer the move-to-side variant. On the other hand, the handshape change variant is the most favoured form for OMS in TWENTY-SEVEN (Figure 5.51), even though it is not commonly used by OMS in the other three number signs. This is probably linked to the finger closed feature of the 7-handshape (see Figure 5.47 on page 165) which make the handshape change from 2 to 7 easier to articulate than closing the 2-handshape and then forming a 7-handshape in the TWENTYplus variant. In fact, a similar school-differentiated pattern is also observed in TWENTYTHREE and TWENTY-EIGHT, though it is not statistically significant.

In sum, TWENTY-ONE favours the TWENTYplus form, TWENTY-THREE and TWENTY-FIVE are more likely to be associated with the handshape change forms, and the wrist-twist variants are favoured by TWENTY-TWO and TWENTY-SEVEN. For the TWENTYplus forms, closing a 2-handshape then open the index finger (i.e., a 1-handshape) is rather effortless as compared with opening to a 3-, 5-, or 8-handshape. It seems that handshape is playing a role in constraining the type of movement involved in these number signs. Moreover, despite the similarity, the handshape change form seem to be more economical than the move-to-side form. This may explain why the former is more popular than the latter in most of the twenties signs except for TWENTY-TWO, which does not require a handshape 
change.

\subsubsection{Age}

Many lexemes also exhibit age differences. Although such a difference may reflect the school effect to a certain extent (due to the connection between age and school discussed in Chapter 4), examples of generational differences that seem to supercede school attendance are also observed. Table 5.6 summarises how many variants are used uniquely by the different age groups. For example, row 1 refers to variants unique to the older age group, whereas row 4 refers to variants uniquely shared by both the older and the middle-aged groups. This will provide us with a baseline for more detailed examination of generational differences in the remainder of this section.

\begin{tabular}{llcc}
\hline & & No. of variants & Items involved \\
\hline 1. & Variants unique to Older age group & 15 & 13 \\
2. & Variants unique to Middle age group & 14 & 13 \\
3. & Variants unique to Younger age group & 10 & 9 \\
4. & Variants unique to Older \& Middle age groups & 20 & 16 \\
5. & Variants unique to Middle \& Younger age groups & 48 & 34 \\
6. & Variants unique to Older \& Younger age groups & 23 & 17 \\
\hline
\end{tabular}

Table 5.6: Variants (both separate and subvariants) unique to age group

The middle and younger age groups have the most shared variants (row 5 of Table 5.6). This may be linked to the fact that they all learnt to sign from their peers in oral schools, whereas most of the older signers learnt to sign from their teachers in signing schools. This break in schooling and language transmission makes it unsurprising that middle and younger signers share more variants. On the other hand, the younger 
age group produced the fewest unique variants (row 3 of Table 5.6). This may indicate that the middle-aged signers have passed along some of their variants to the younger signers. Therefore, the latter did not have to create as many variants as the former.

It is interesting to see there are variants unique to the older and younger age groups (row 6 of Table 5.6). Nonetheless, most of these variants are produced by children of deaf adults and their parents, and they are mostly minor variants made by five participants or less.

When we look at the total number of variants made by each age group across different categories, we can see that the older age group produce comparatively fewer variants than the other two groups (see Table 5.7). This discrepancy is most prominent in the number signs. While 67 variants are used for 18 number signs by the older signers, the middle and younger age groups produced 94 and 86 variants respectively. Again, this may be linked to the education received by the different groups. Because most of the older participants learnt signs from their teachers in school, their lexicons have less variation than the other two age groups.

\begin{tabular}{lrrr}
\hline Categories & Younger & Middle & Older \\
\hline Colour & 57 & 52 & 49 \\
Kinship & 14 & 14 & 12 \\
Country/region & 40 & 42 & 44 \\
Number & 86 & 94 & 67 \\
\hline Total & 198 & 201 & 173 \\
\hline
\end{tabular}

Table 5.7: Number of variants by categories and age groups

Individual items further affirm that there is some age differentiation. Some known 'old' signs are mainly used by the middle-aged and/or the older signers. For example, no younger signers produced AUSTRALIA3 or 
BLACK2; PURPLE3 is only used by the older signers and one younger signer who has deaf parents (see Table 5.8 for the variants illustration and the distribution of the variants by age groups). In addition, these 'old' signs are only used by $20 \%$ or less of the middle-aged and the older participants (9\% to $20 \%$ ). This implies that many older signers opt for newer forms rather than keeping the old ones. It is very likely that the 'old' variants will disappear in the future.

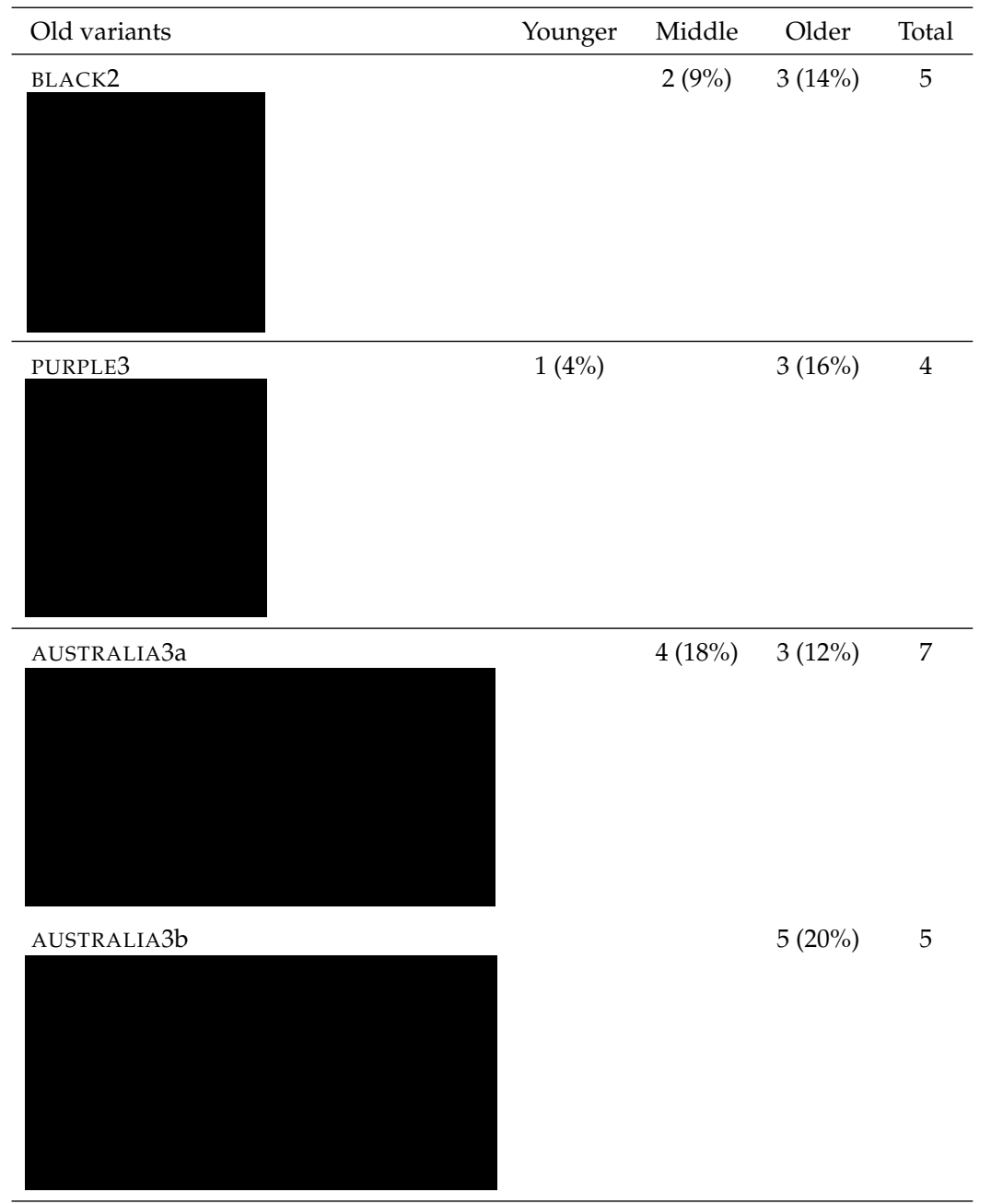

Table 5.8: Examples of old variants and their frequency distribution by age

While some old forms are fading away, there are new forms, which 
are building on the old ones. Two typical examples are RED and GREEN. Tables 5.9 and 5.10 lay out the frequencies of the major variants by age groups of RED and GREEN respectively. ${ }^{7}$ REDa is the most frequent variant (used by $55 \%$ of all participants), which is also the 'citation form' found in the HKSL publications as mentioned earlier. As we can see in Table 5.9 , both participants of the middle-aged and the older groups prefer this variant (67\% in both groups). In contrast, the younger signers favour REDb (48\%) more than REDa (35\%). A similar pattern is seen in GREEN. While the older signers tend to use more GREENa (67\%) and the middle-aged signers seem to have no preference between GREENa (38\%) and GREENb (38\%), the younger signers like to use GREENC (44\%) more than the other two variants ( $4 \%$ for GREENa and 26\% for GREENb). In both RED and GREEN, the innovative forms (i.e., REDb and GREENC) are clearly favoured by the younger participants and these forms are obviously derived from the 'old' variants. The location of REDb is lowered from lip (the location of the 'old' sign) to chin, whereas the palm orientation of GREENC has changed from facing in/out of the signer to facing down. ${ }^{8}$ It seems that in both variants, the original iconicity has become opaque. For RED, touching the lip by the index finger to signify red is the origin of the sign. For GREEN, the wiggling of two upright fingers (index and middle fingers) imitates the appearance and movement of grass. Indeed, the 'old' variant also means grass. By changing the palm orientation of the sign, the two fingers are facing out instead of upright, the sign also loses its iconicity.

\footnotetext{
${ }^{7}$ The $\mathrm{p}$ values shown are the results of Fisher's Exact tests conducted on the data.

${ }^{8}$ The most frequent form in the dataset is GREENa (the one with palm facing out) and the HKSL publications use GREENb (the one with palm facing in) more often. It is not clear which one is the original form.
} 


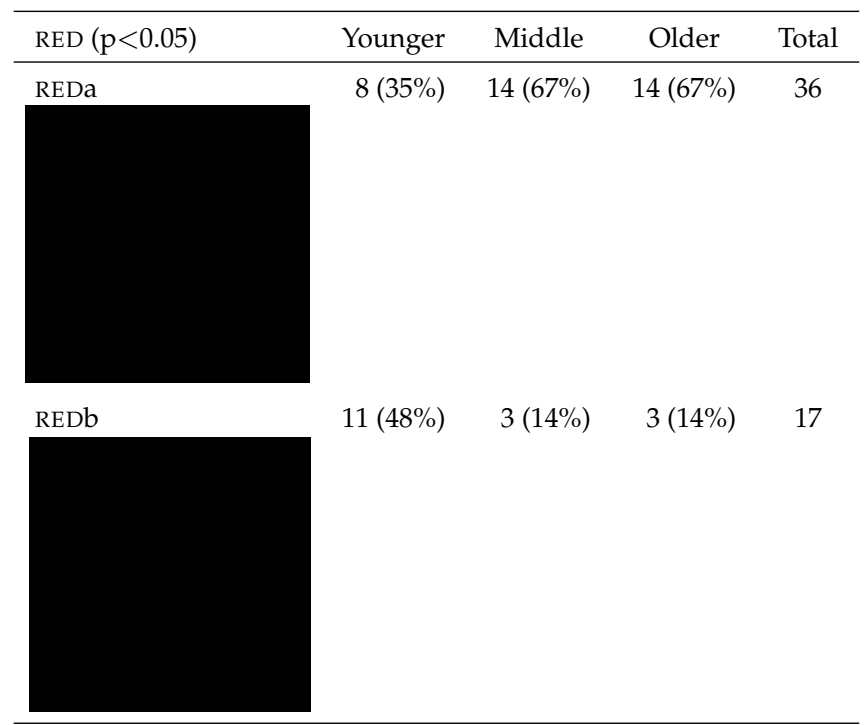

Table 5.9: Frequency distribution of major variants of RED by age

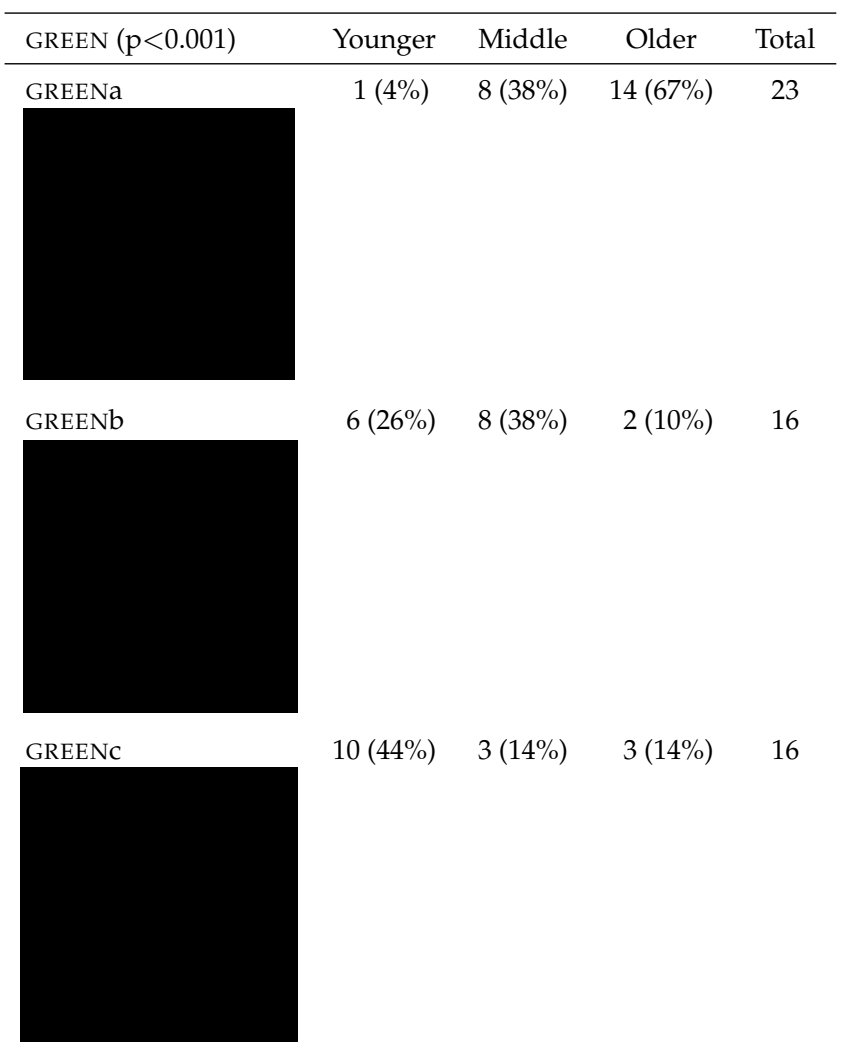

Table 5.10: Frequency distribution of major variants of GREEN by age 
Other colour signs demonstrating significant age-differentiation are PINK and ORANGE. Over half (54\%) of the younger participants produced the innovative form PINK1a and its subvariant PINK1b, while the middle-aged group seems to have no preference and the older group slightly favours the old variant, PINK4, which is a compound of RED and LITTLE. Table 5.11 displays the frequency distribution of the major variants with subvariants grouped together (see Figure 5.6 on page 136 for sign illustrations). It is worth noting that all variants PINK2, PINK3, and PINK4 are compound signs and their subvariants are grouped together.

\begin{tabular}{lrrcr}
\hline PINK $(\mathrm{p}<0.0001)$ & Younger & Middle & Older & Total \\
\hline PINK1 & $13(54 \%)$ & $6(29 \%)$ & $1(5 \%)$ & 20 \\
PINK2 & $1(4 \%)$ & $6(29 \%)$ & $6(27 \%)$ & 13 \\
PINK3 & $2(8 \%)$ & $6(29 \%)$ & $3(14 \%)$ & 11 \\
PINK4 & $2(8 \%)$ & & $8(36 \%)$ & 10 \\
\hline
\end{tabular}

Table 5.11: Frequency distribution of major variants of PINK by age

In ORANGE, the younger signers prefer ORANGE2a over the most frequent variant ORANGE1 (31 out of 66 tokens, i.e., $47 \%$ of the total), which is favoured by both the middle-aged and the older groups. The frequency distribution of the two variants is tabulated in Table 5.12 (see Figure 5.13 on page 139 and Figure 5.14a on page 140 for sign illustrations). As explained in Section 5.4.1.1, ORANGE2a is a one-handed form derived from a two-handed sign resembling the action of eating an orange. The twohanded variant ORANGE2b is only produced by three older signers (14\%) and neither the middle-aged nor the younger signers use it.

\begin{tabular}{lrrcr}
\hline ORANGE $(\mathrm{p}<0.05)$ & Younger & Middle & Older & Total \\
\hline ORANGE1 & $6(25 \%)$ & $16(76 \%)$ & $9(43 \%)$ & 31 \\
ORANGE2a & $12(50 \%)$ & $4(19 \%)$ & $5(24 \%)$ & 21 \\
\hline
\end{tabular}

Table 5.12: Frequency distribution of major variants of ORANGE by age 
The new forms building on an early form seem to be more acceptable to the older signers, but some innovative forms are not very welcome. A commonly known new form of the number ELEVEN has received much criticism because it has another meaning in the lexicon of the older generation. The handshape with the index and pinky fingers extended refers to one-and-a-half for the older signers, but some younger and middle-aged signers use it for ELEVEN. In the dataset, there are three variants of this new form, palm-in (ELEVEN1b), palm-out (ELEVEN1c) (see Figure 5.52), and a wrist-twist form (ELEVEN1d) and they altogether comprise just 16\% of the total (only one young male signer made ELEVEN1d). None of the older participants use such form, 64\% are produced by the middle-aged signers and $36 \%$ by the younger individuals. This may indicate that the new form was innovated in the middle-aged generation but it has not widely spread due to the resistance from the older generation and a general dispreference for change that creates homonyms (Labov, 1994).

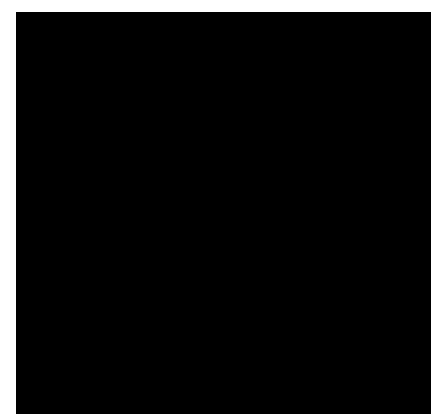

(a) ELEVEN1b

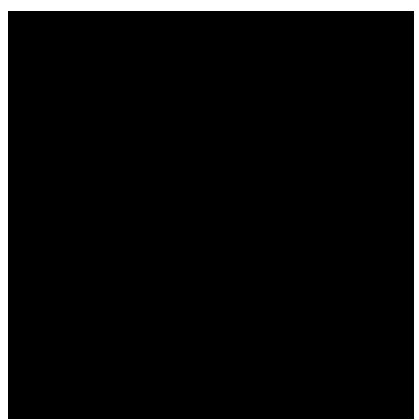

(b) ELEVEN1c

Figure 5.52: New forms of ELEVEN

With regard to other number signs, age related variants in the twenties seem to mirror those found in school-differentiated variants. The frequency distribution of TWENTY-ONE, TWENTY-THREE, TWENTY-FIVE, and TWENTY-SEVEN by age groups is tabulated in Table 5.13 (see Figure 5.46 
on page 165 for the exemplification of TWENTY-ONE for the various forms). Although the older signers tend to favor the TWENTYplus form in TWENTYONE and TWENTY-THREE (77\% for the first and $43 \%$ for the second), this is not the case for TWENTY-FIVE (33\%) and TWENTY-SEVEN (14\%). As the wrist-twist form gains popularity in TWENTY-SEVEN as discussed earlier, the older signers prefer the handshape change form (36\%). While the middle-aged signers seem to hover between the handshape change forms and the move-to-side variants, the younger signers have a strong liking for the handshape change variants except for TWENTY-SEVEN, in which both the younger and middle-aged signers prefer the wrist-twist form. This may indicate that the younger signers are shifting to the handshape change form for reasons of economy suggested earlier.

\begin{tabular}{lrrrr}
\hline Numbers & Younger & Middle & Older & Total \\
\hline Twenty-one $(\mathrm{p}<0.0001)$ & & & & \\
TWENTY-ONEhandshape & $11(48 \%)$ & $7(33 \%)$ & $1(5 \%)$ & 19 \\
TWENTY-ONEmoveSide & $4(17 \%)$ & $7(33 \%)$ & $2(9 \%)$ & 13 \\
TWENTY-ONEtwist & $3(13 \%)$ & $2(10 \%)$ & $1(5 \%)$ & 6 \\
TWENTYplus & $4(17 \%)$ & $3(14 \%)$ & $17(77 \%)$ & 24 \\
Twenty-three (p<0.01) & & & & \\
TWENTY-THREEhandshape & $16(70 \%)$ & $10(48 \%)$ & $6(29 \%)$ & 32 \\
TWENTY-THREEmoveSide & $2(9 \%)$ & $8(38 \%)$ & $6(29 \%)$ & 16 \\
TWENTYplus & $3(13 \%)$ & $1(5 \%)$ & $9(43 \%)$ & 13 \\
Twenty-five (p<0.01) & & & & \\
TWENTY-FIVEhandshape & $14(61 \%)$ & $9(45 \%)$ & $3(14 \%)$ & 26 \\
TWENTY-FIVEmoveSide & $3(13 \%)$ & $7(35 \%)$ & $9(43 \%)$ & 19 \\
TWENTY-FIVEtwist & $1(4 \%)$ & $1(5 \%)$ & $1(5 \%)$ & 3 \\
TWENTYplus & $3(13 \%)$ & $1(5 \%)$ & $7(33 \%)$ & 11 \\
Twenty-Seven (p<0.01) & & & & \\
TWENTY-SEVENhandshape & $7(30 \%)$ & $3(14 \%)$ & $8(36 \%)$ & 18 \\
TWENTY-SEVENmoveSide & $2(9 \%)$ & $4(19 \%)$ & $4(18 \%)$ & 10 \\
TWENTY-SEVENtwist & $12(52 \%)$ & $12(57 \%)$ & $1(5 \%)$ & 25 \\
TWENTYplus & $1(4 \%)$ & & $3(14 \%)$ & 4 \\
\hline & & & & \\
\hline
\end{tabular}

Table 5.13: Frequency distribution of twenties by age 
Turning to the kinship terms, this appears to be the most stable category as stated earlier. Each of the terms has a highly frequent 'citation form' and an innovative form, except for RELATIVES. Table 5.14 displays the frequencies of the 'citation form' and innovative forms of these six kinship terms by age groups. Although the number of counts do not show significant differences across all kinship terms, it is clear that the younger and the middle-aged signers produced more innovative forms than the older signers in FATHER, MOTHER, ELDER-SISTER, and YOUNGER-SISTER. Particularly, there is no older signers produced the new forms for both FATHER and MOTHER.

\begin{tabular}{lrrrr}
\hline Kinship terms & Younger & Middle & Older & Total \\
\hline Father & & & & \\
FATHERa & $20(87 \%)$ & $17(81 \%)$ & $21(100 \%)$ & 58 \\
FATHERb & $3(13 \%)$ & $4(19 \%)$ & & 7 \\
Mother & & & & \\
MOTHERa & $18(78 \%)$ & $16(76 \%)$ & $21(100 \%)$ & 55 \\
MOTHERb & $4(17 \%)$ & $5(24 \%)$ & & 9 \\
Elder brother & & & & \\
ELDER-BROTHER1 & $17(71 \%)$ & $13(62 \%)$ & $13(62 \%)$ & 43 \\
ELDER-BROTHER2 & $6(25 \%)$ & $6(29 \%)$ & $6(29 \%)$ & 18 \\
Elder sister & & & & \\
\hline ELDER-SISTER1 & $18(78 \%)$ & $16(73 \%)$ & $17(81 \%)$ & 51 \\
ELDER-SISTER2 & $4(17 \%)$ & $5(23 \%)$ & $3(14 \%)$ & 12 \\
Younger brother & & & & \\
YOUNGER-BROTHER1 & $14(56 \%)$ & $12(57 \%)$ & $11(52 \%)$ & 37 \\
YOUNGER-BROTHER2 & $10(40 \%)$ & $8(38 \%)$ & $8(38 \%)$ & 26 \\
\hline Younger Sister & & & & \\
\hline YOUNGER-SISTER1 & $13(54 \%)$ & $13(62 \%)$ & $16(76 \%)$ & 42 \\
YOUNGER-SISTER2 & $10(42 \%)$ & $7(33 \%)$ & $5(24 \%)$ & 22 \\
\hline
\end{tabular}

Table 5.14: Frequency distribution of kinship terms by age

In addition, it is noticeable that the signs for younger brother and younger sister are more prone to change than elder brother and elder sister. It may be 
due to the fact that the sign YOUNGER is sometimes used as a name sign or part of a name sign for a deaf person who has an elder deaf sibling. In other words, a deaf person may be named by adding YOUNGER to the name sign of his/her elder sibling, but not the other way round. Therefore, while both ELDER and YOUNGER are bound morphemes (Figure 5.53), the latter may have undergone grammaticalization or be at a further stage of the process because of its expanded usage in everyday discourse.

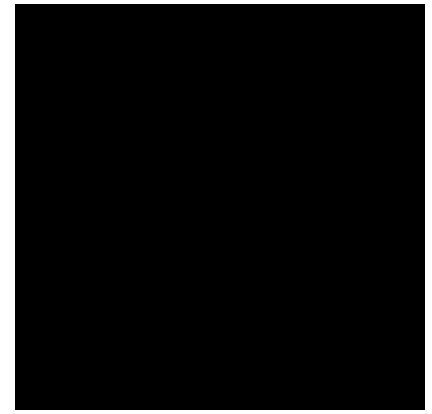

(a) ELDER

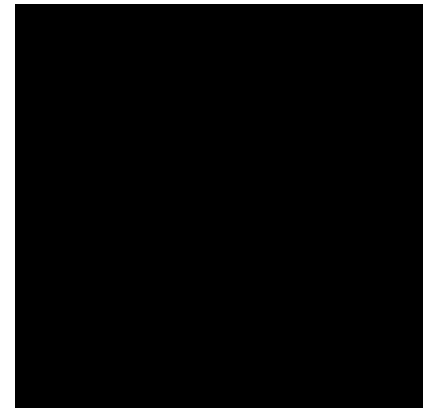

(b) YOUNGER

Figure 5.53: Bound morphemes in sibling terms

As mentioned earlier, seven out of the eight country / region signs have a variant that is the country/region's own local sign. In TAIWAN, TAIWAN1 is Taiwan's local sign, which resembles the action of eating a sugarcane. It is predominantly used by the younger and middle-aged groups (46\% and $64 \%$ respectively as shown in Table 5.15 ). But only $22 \%$ of the older signers produced TAIWAN1. Many of them prefer the compound sign TAIWAN2a (61\%), a Hong Kong sign for TAIWAN which is believed to have a longer history. It is also recorded in the first HKSL book published in 1972 and three subsequent publications (see Appendix D, Figure 44). The other two variants are minor ones, but both of them have connection with TAIWAN2a. TAIWAN2b (Figure 5.54a) is a one-handed reduced subvariant $(10 \%$ of the total) and TAIWAN3 (Figure 5.54b) is another reduced version 
that drops the first part of the compound ( $3 \%$ of the total).

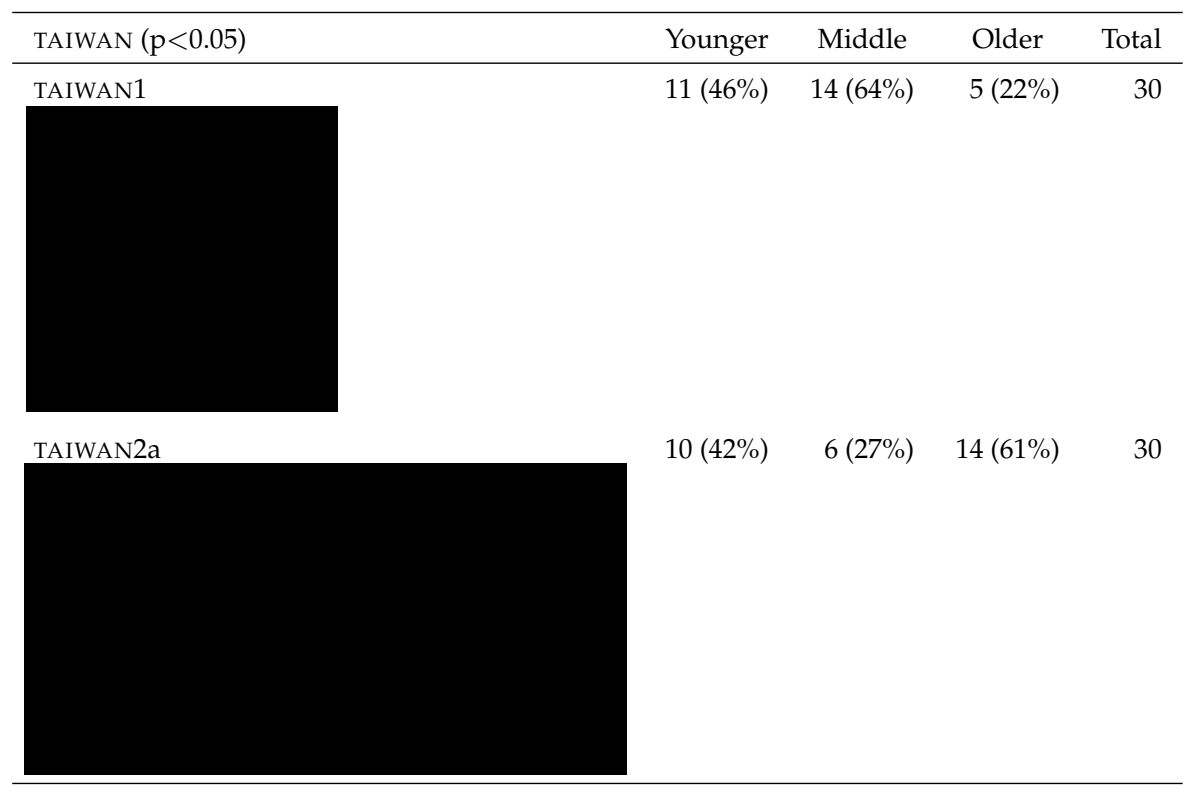

Table 5.15: Frequency distribution of major variants by age: TAIWAN

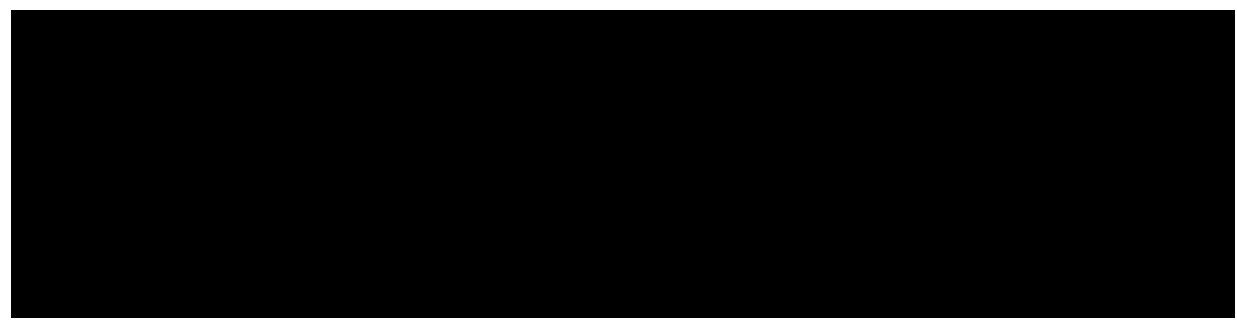

(a) TAIWAN2b

(b) TAIWAN3

Figure 5.54: Minor variants of TAIWAN

By contrast, the region's own local sign BEIJING1b seems to have been part of the HKSL lexicon ever since the language emerged. The first HKSL book and three subsequent publications have this form as the only entry for BEIJING (see Appendix D, Figure 39). This variant was probably brought to Hong Kong by the deaf people from mainland China in the early days as described in Chapter 3. This form has a subvariant 
BEIJING1a which is supposed to be derived from the original form BEIJING1b. While BEIJING1b moves the 2-handshape diagonally across the torso from the upper chest to near the waist area, BEIJINGla moves the 2-handshape across the upper chest horizontally (see Figures 5.32a and $5.32 \mathrm{~b}$ on page 152 for sign illustrations). If we just look at the proportion of these two related variants in Table 5.16, we can see that the older signers mainly use the original form (6 versus 1 ) whereas the middle-aged and the younger groups tend to use the new form (6 versus 2 and 5 versus 3 respectively). Although BEIJING1 (combining BEIJING1a and BEIJING1b) is the most frequent variant, it only comprises about $38 \%$ of the total. ${ }^{9}$ This means that this variant may have lost its popularity to BEIJING2 (combining two subvariants with different handshapes as illustrated in Figures 5.32c and 5.32d on page 152) over time. BEIJING2 is very likely a locally created Hong Kong sign, which simply makes the character sign 北 (literally means north, the first character in the Chinese name of Beijing). It is slightly favoured by the younger group, and $43 \%$ of them produced it. The middle-aged and the older group both prefer BEIJING1 (36\% and 29\% respectively) over BEIJING2 (27\% and $21 \%$ respectively). Interestingly, the most popular variant among the older signers is the compound of BEIJING1 and BEIJING2, i.e., BEIJING3. Nearly half (46\%) of the older signers chose it. This may suggest that the older signers recognize the new variant, but at the same time, they do not want to abandon their old variant. Hence, they formed a compound of the two forms.

\footnotetext{
${ }^{9}$ There are two more related subvariants produced by only one and two signers. The calculation of the percentage included these minor subvariants.
} 


\begin{tabular}{lcccr}
\hline BEIJING $(\mathrm{p}<0.05)$ & Younger & Middle & Older & Total \\
\hline BEIJING1a & $5(22 \%)$ & $6(27 \%)$ & $1(4 \%)$ & 12 \\
BEIJING1b & $3(13 \%)$ & $2(9 \%)$ & $6(25 \%)$ & 11 \\
BEIJING2a & $9(39 \%)$ & $2(9 \%)$ & $3(13 \%)$ & 14 \\
BEIJING2b & $1(4 \%)$ & $4(18 \%)$ & $2(8 \%)$ & 7 \\
BEIJING3a & $2(9 \%)$ & $4(18 \%)$ & $6(25 \%)$ & 12 \\
BEIJING3b & & $1(5 \%)$ & $5(21 \%)$ & 6 \\
\hline
\end{tabular}

Table 5.16: Frequency distribution of major variants by age: BEIJING

Regarding the country signs, Australia's own country sign has also found its way to Hong Kong. Table 5.17 demonstrates the frequency distribution of the major variants of AUSTRALIA. AUSTRALIA1 is the most frequent variant which imitates a kangaroo to signify Australia, which is very likely a locally created Hong Kong sign. AUSTRALIA2a is the sign used in Auslan to represent its country and AUSTRALIA2b is its subvariant. ${ }^{10}$ The figures show that AUSTRALIA2 is favoured by the younger signers, with half of the younger signers using it (combining AUSTRALIA2a and AUSTRALIA2b). Both the middle-aged and the older groups prefer AUSTRALIA1 (55\% and 56\% respectively). The older signers also like to use the 'old variant' (AUSTRALIA3) mentioned earlier (see Table 5.8 on page 171) rather than this borrowed variant ( $32 \%$ as opposed to $4 \%$ ).

${ }^{10}$ The handshape difference in AUSTRALIA2b is very likely a by-product of borrowing. 


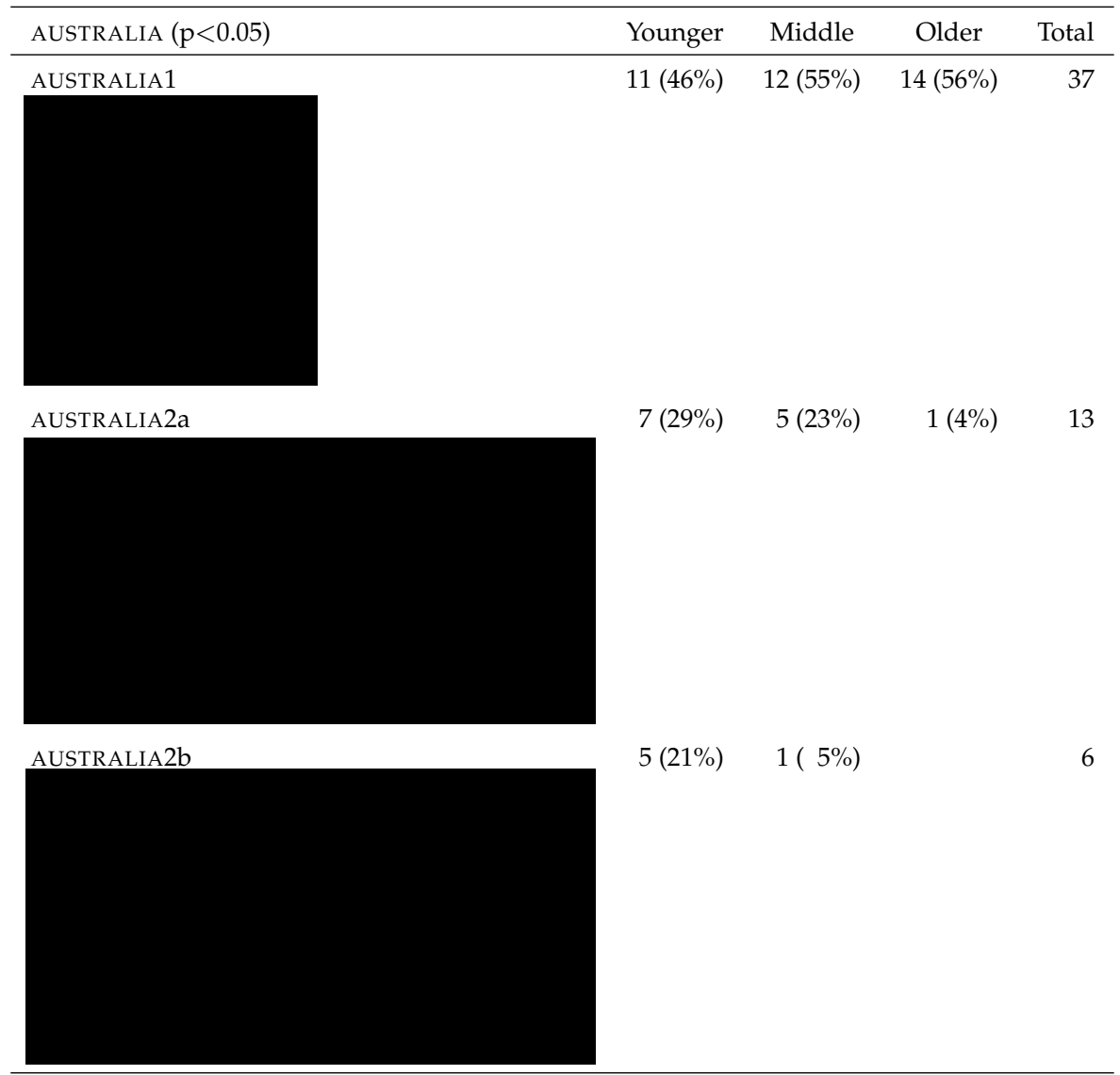

Table 5.17: Frequency distribution of major variants by age: AUSTRALIA

Sometimes, it is not easy to distinguish whether a sign is borrowed from the country's own sign language or a derived variant of a local sign due to the iconic similarity among different sign languages. INDIA is a good example. INDIA2a is articulated by pointing the index finger to the centre of forehead to symbolize the bindi on Indian women and INDIA2b is a subvariant substituting the index finger with the thumb (see Figure 5.33 on page 153 for sign illustrations). It happened that INDIA2b is also the sign for India in Indian Sign Language. Although it is hard to know whether INDIA2b is a locally derived new form or a borrowing from Indian Sign Language, it is clear that INDIA2 (combining INDIA2a and INDIA2b) is 
disfavoured by the older group. As shown in Table 5.18, the older signers like to use INDIA1, which symbolizes the turban worn by Indian men. ${ }^{11}$ On the other hand, the younger group prefers INDIA2 (50\%) more than INDIA1 (27\%) and the middle-aged group slightly favours INDIA2 (45\% as opposed to $40 \%)$.

\begin{tabular}{lcccr}
\hline INDIA $(\mathrm{p}<0.01)$ & Younger & Middle & Older & Total \\
\hline INDIA1 & $6(27 \%)$ & $8(40 \%)$ & $16(67 \%)$ & 30 \\
INDIA2a & $5(23 \%)$ & $4(20 \%)$ & $2(9 \%)$ & 11 \\
INDIA2b & $6(27 \%)$ & $5(25 \%)$ & & 11 \\
\hline
\end{tabular}

Table 5.18: Frequency distribution of major variants by age: INDIA

Lastly, a more recent borrowing country sign is ITALY4 (Figure 5.55). However, this variant is only produced by the middle-aged participants. Neither the older nor the younger signers used such variant. This is not a minor variant as $29 \%$ of the middle-aged participants produced it and it comprises $11 \%$ of the total. This may be due to the origin of this variant. I speculate that the temporary stay of an Italian deaf man in 2006 is the source. During his stay, he would have introduced his country sign to the Hong Kong deaf people he had contact with, and they are mainly in the middle-aged group. Moreover, the dissemination of this variant may be hindered by the younger signers who are perhaps more proud of their deaf identity and their language. I was once asked by a young signer about my opinion on using a country's own local sign instead of the HKSL sign for the country when interpreting. This young signer complained about an interpreter who chose to use a country's own local sign instead of an

\footnotetext{
${ }^{11}$ The minor subvariant INDIA1b (different in handshape) is grouped together for calculation.
} 
existing Hong Kong sign in an open interpreting situation. She explained, "as a HKSL interpreter, one should use Hong Kong signs." This may be a single case, but the strong sense of deaf identity together with a positive attitude towards their language among the younger generation certainly exist and continue to spread. As described in Chapter 3, the deaf staff of the Centre for Sign Linguistics and Deaf Studies (CSLDS) play an important role in promoting the value of their own language and identity. It could also be attributed to the growing promotion of HKSL in the media, which fosters a more open and positive attitude towards HKSL in the general public. It would not be surprising to see other deaf people, not only the younger ones (although they may be the most likely to), resisting the import of foreign signs into the HKSL lexicon.

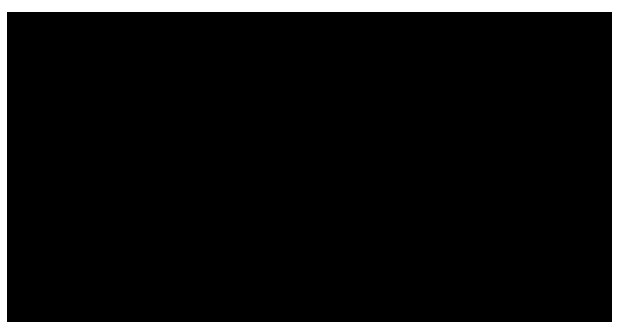

Figure 5.55: ITALY4

\subsubsection{Gender}

Compared with school and age, gender differentiation in the choices of variants is less strong. There are no variants unique to either men or women in the kinship domain, but there are eight forms in each of the colour and country/region domains that appear to be gender specific. These sixteen variants are found in five colour terms (BLUE, ORANGE, PINK, PURPLE, and SILVER) and seven country/region names (BEIJING, GUANGZHOU, IN- 
DIA, ITALY, KOREA, TAIWAN, and VIETNAM). Ten of them are unique to men and six are unique to women. They are all minor variants produced by two to five participants.

\begin{tabular}{lrr}
\hline & Female & Male \\
\hline ELDER-SISTER $(\mathrm{p}=0.639)$ & & \\
ELDER-SISTER1 & $29(81 \%)$ & $22(73 \%)$ \\
ELDER-SISTER2 & $5(14 \%)$ & $7(23 \%)$ \\
ELDER-BROTHER $(\mathrm{p}<0.05)$ & & \\
ELDER-BROTHER1 & $27(77 \%)$ & $16(52 \%)$ \\
ELDER-BROTHER2 & $5(14 \%)$ & $13(42 \%)$ \\
YOUNGER-SISTER $(\mathrm{p}<0.05)$ & & \\
YOUNGER-SISTER1 & $27(77 \%)$ & $15(48 \%)$ \\
YOUNGER-SISTER2 & $7(20 \%)$ & $15(48 \%)$ \\
YOUNGER-BROTHER $(\mathrm{p}<0.01)$ & & \\
YOUNGER-BROTHER1 & $25(71 \%)$ & $12(38 \%)$ \\
YOUNGER-BROTHER2 & $8(23 \%)$ & $18(56 \%)$ \\
\hline
\end{tabular}

Table 5.19: Frequency distribution of four sibling terms by gender

Although there are no variants unique to either females or males among the kinship terms, three terms are found to be significantly preferred by one gender over the other, and they are ELDER-BROTHER, YOUNGER-BROTHER, and YOUNGER-SISTER (see Appendix D, Figures 19, 21, and 22 for sign illustrations). As shown in Table 5.19, more men used the new forms than women in all four sibling terms, though this trend is not significant in the term ELDER-SISTER. While women produced a relatively similar proportion of the new forms in all four terms (between 14\% and 23\%), the proportion of these new variants produced by men increased gradually from 23\% for ELDER-SISTER to $56 \%$ for YOUNGER-BROTHER.

Another point to note in these examples of gender effects is the conservative attitude of women as opposed to men. In fact, in FATHER and MOTHER, similar gender differentiation is observed. The new forms FA- 
THERb and MOTHERb accounted for $11 \%$ and $14 \%$ of the sample respectively, and male signers produced $71 \%$ of FATHERb and $67 \%$ of MOTHERb (see Figure 5.22 on page 145 for sign illustrations). Though the differences are not statistically significant as they are for some of the sibling terms, they show a congruous pattern that reflects the conservative behaviour in language use by women (Bayard, 1989; Pan, 1981; Yeung, 1980), particularly in the use of kinship terms.
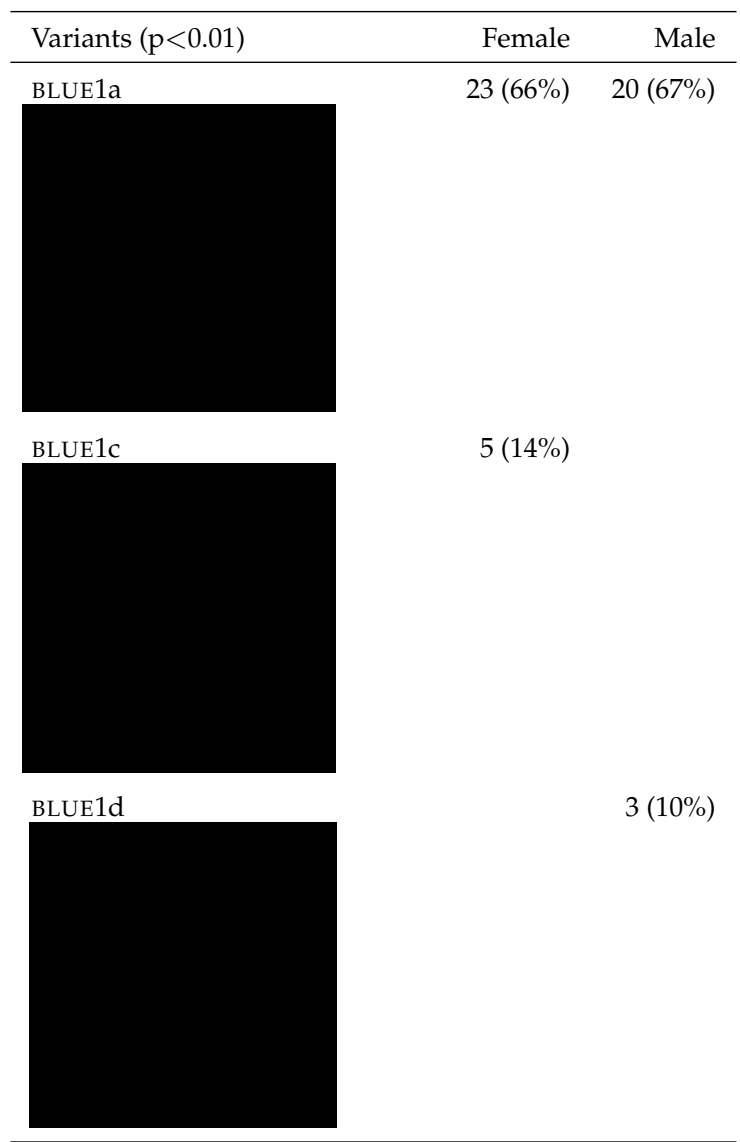

$5(14 \%)$

Table 5.20: Frequency distribution of variants of BLUE1 by gender

For the 12 items that have female or male unique variants, only the colour sign BLUE demonstrates statistical significance. Table 5.20 illustrates the most frequent variant and the two subvariants unique to fe- 
male and male, together with their relative percentages and the result of Fisher's Exact test. It is not clear why men and women would develop different sublexical forms of BLUE. It is apparent that both forms involve a change in handshape from the original form. Further research on the handshapes used by different genders may offer some explanation.

Indeed, BLUE is not the only sign that exhibit a handshape difference between men and women. In KOREA1 (see Figure 5.36 on page 155 for sign illustrations), the variant imitating the traditional Korean dress has three subvariants using different handshapes: L-handshape in KOREA1a, flat-handshape in KOREA1d, and aeroplane-handshape in KOREA1e. The L-handshape variant is the most common one and $67.5 \%$ are produced by women. ${ }^{12}$ While the flat-handshape is more balanced across men and women (44.4\% and 55.6\% respectively), only men produced the aeroplanehandshape variant.

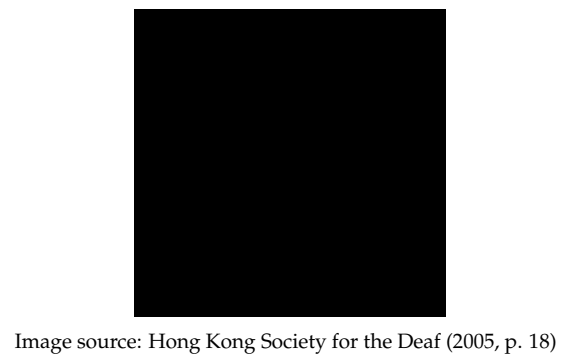

(a) L-handshape

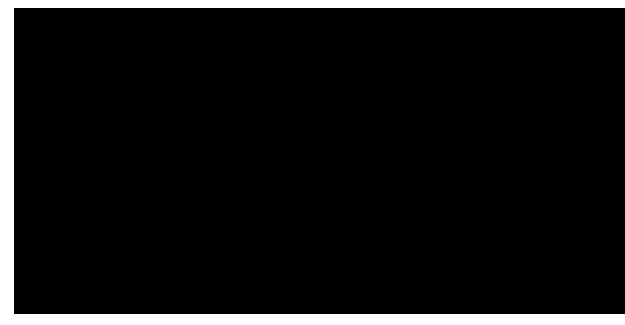

Image source: Tang (2007, p. 355)

(b) Aeroplane-handshape

Figure 5.56: Phonological variants of NEW

It is interesting that no women used the aeroplane-handshape variant because L-handshape and aeroplane-handshape are not contrastive

\footnotetext{
${ }^{12}$ There are three subvariants of the L-handshape variant. The denominator is the combination of all three subvariants.
} 
in other signs like NEW (Figure 5.56) and NORTH (see Figures 5.32c and $5.32 \mathrm{~d}$ on page 152). This means both signs can be articulated with either a L-handshape or an aeroplane-handshape without altering their meanings. Returning to the 'dress' variants of KOREA (i.e., KOREA1), the fact that only men made the aeroplane-handshape variant may signify that women consider this handshape as inappropriate to simulate a dress from a dress wearer's perspective. For the men, they may not be as mindful as women and it may also suggest that the iconicity of this variant is waning, especially in the men's mind. Supporting evidence for this can be seen in one of the L-handshape subvariants for which a reverse movement is observed (see Figure 5.36c on page 155). Instead of moving downwards to trace the shape of a dress, the movement in this variant is upwards, which made the iconicity of the sign opaque. This upwards movement variant (KOREA1C) is largely made by men (75\%).

\begin{tabular}{|c|c|c|}
\hline & Female & Male \\
\hline \multicolumn{3}{|c|}{ THREE $(\mathrm{p}=0.052)$} \\
\hline \multirow{2}{*}{ 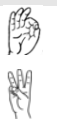 } & $31(91 \%)$ & $21(70 \%)$ \\
\hline & $3(9 \%)$ & $9(30 \%)$ \\
\hline \multicolumn{3}{|c|}{ THIRTEEN $(\mathrm{p}=0.018)$} \\
\hline \multirow{2}{*}{ 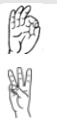 } & $36(100 \%)$ & \\
\hline & & $5(16 \%)$ \\
\hline \multicolumn{3}{|c|}{ TWENTY-THREE $(\mathrm{p}=0.209)$} \\
\hline $\begin{array}{l}\text { 閐 } \\
\text { 暗 }\end{array}$ & $35(100 \%)$ & \\
\hline$(x)$ & & $2(7 \%)$ \\
\hline
\end{tabular}

Table 5.21: Handshape use by gender in THREE, THIRTEEN, and TWENTY-THREE

Another handshape difference between men and women is observed in numbers. Recall that there are two different 3-handshapes, one uses the last three fingers and the other uses the middle three fingers (see Figure 
5.38 on page 157). Although both are widely accepted variants, the lastthree-finger form seems to be more popular (as confirmed by this dataset). It is generally believed that the middle-three-finger form is more common in counting, as it is easier to change from a 2-handshape (the index and the middle fingers) to a middle-three-finger handshape than to a lastthree-finger handshape. It is quite surprising to see that the male signers tend to use more of the middle-three-finger handshape than the female signers. This effect is observed in both THREE and THIRTEEN, but diminished in TWENTY-THREE. As shown in Table 5.21, the number of middlethree-finger handshape used is the highest in THREE, then THIRTEEN and TWENTY-THREE, but women only used it in THREE. The contrasting behaviour of men and women is most significant in THIRTEEN ( $p=0.018)$, is at the edge of significance for THREE $(p=0.052)$, but is not significant in TWENTY-THREE $(p=0.209)$.

Since I could not think of any explanation for these differences from my female perspective, I consulted a male signer to see if he could give me any insight. He suggested that men use the middle-three-finger handshape more frequently than women because the sign WIN/CHAMPION (Figure $5.57)$ is made by this handshape and men tend to talk more about winning and losing, like sports games and horse racing, than women do. This explanation sounds quite reasonable, especially from the perspective of usage-based models of language (Langacker, 1988, 1999). 


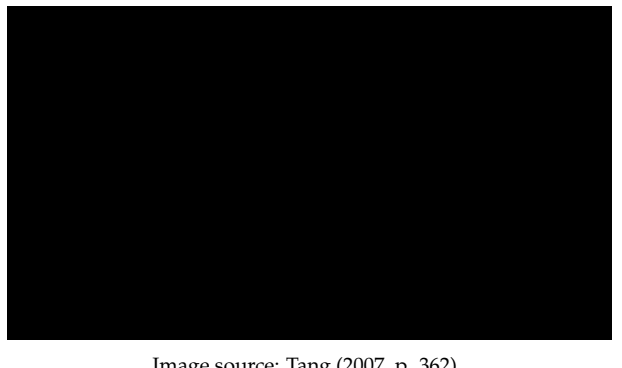

Image source: Tang $(2007$, p. 362)

Figure 5.57: WIN/CHAMPION

In addition, the handshapes for EIGHT demonstrate a similar pattern too. The most frequent handshape is the L-handshape and the other one is the first-three-finger handshape, which is commonly used by the hearing people in Hong Kong (and possibly other Chinese societies) to signify eight (see Figure 5.39b and 5.39c on page 157). The numbers reported in Table 5.22 look akin to those presented above regarding the 3-handshape. The $\mathrm{p}$-value in EIGHTEEN is the most significant ( $\mathrm{p}=0.037)$, EIGHT is on the borderline $(\mathrm{p}=0.051)$, and TWENTY-EIGHT is far from significant $(\mathrm{p}=0.493)$. Again, the reason for this is something of a mystery. Yet, if we apply the usage-based theory like the case in THREE, the most exemplary sign of the first-three-finger handshape is GOVERNMENT (see Figure 4.11 on page 97). The meaning of this sign is not limited to signify the government but is also often used to refer to figures in authority. Some of the domains in which power and authority are more likely to be talked about may also be more 'masculine' domains. Some work domains or work-related topics may also be conventionally more masculine (Holmes \& Stubbe, 2003). This means the sign GOVERNMENT may simple occur more often among men than they do among women. 


\begin{tabular}{|c|c|c|}
\hline & Female & Male \\
\hline \multicolumn{3}{|c|}{ EIGHT $(p=0.051)$} \\
\hline \multirow{2}{*}{$\begin{array}{l}\text { है? } \\
\text { है? }\end{array}$} & $32(91 \%)$ & $21(70 \%)$ \\
\hline & $3(9 \%)$ & $9(30 \%)$ \\
\hline \multicolumn{3}{|c|}{ EIGHTEEN $(\mathrm{p}=0.037)$} \\
\hline \multirow{2}{*}{$\begin{array}{l}\text { की } \\
\text { की }\end{array}$} & $33(94 \%)$ & $23(74 \%)$ \\
\hline & $2(6 \%)$ & $8(26 \%)$ \\
\hline \multicolumn{3}{|c|}{ TWENTY-EIGHT $(p=0.493)$} \\
\hline से? & $31(89 \%)$ & $24(80 \%)$ \\
\hline ? & $4(11 \%)$ & $6(20 \%)$ \\
\hline
\end{tabular}

Table 5.22: Handshape use by gender in EIGHT, EIGHTEEN, and TWENTY-EIGHT

Nonetheless, THREEc/d (middle-three-finger handshape) and EIGHTb/c (first-three-finger handshape) are only minor variants with at most $19 \%$ of the participants producing them. The explanation given above is just a preliminary speculation. More research is required to test such claims. In fact, a gender difference in adopting handshape has been observed in several lexical items (including BLUE and KOREA discussed earlier). This suggests a possible future research direction in gender and language.

Finally, pink and purple (see Figure 5.6 on page 136 and Figure 5.17 on page 142 for sign illustrations) are generally perceived as feminine colours. When we look at the frequency distribution of their variants, the distinct tendency of men and women is revealed. Figures 5.58 and 5.59 illustrate the percentage distribution of all the variants (subvariants are combined) of PINK and PURPLE used by the two genders respectively. We can see that the majority of the women tend to use PINK1 or PINK2 and PURPLE1, i.e., focusing on one or two variants. However, the men demonstrate a more widely distributed pattern in their choice of PINK. Even though more than half of the men favour PURPLE1, 20\% of them produced an idiosyn- 
cratic variant, and these are grouped as PURPLE9_others. They also produced a higher percentage of unique forms of PINK (PINK9_others) than the women.

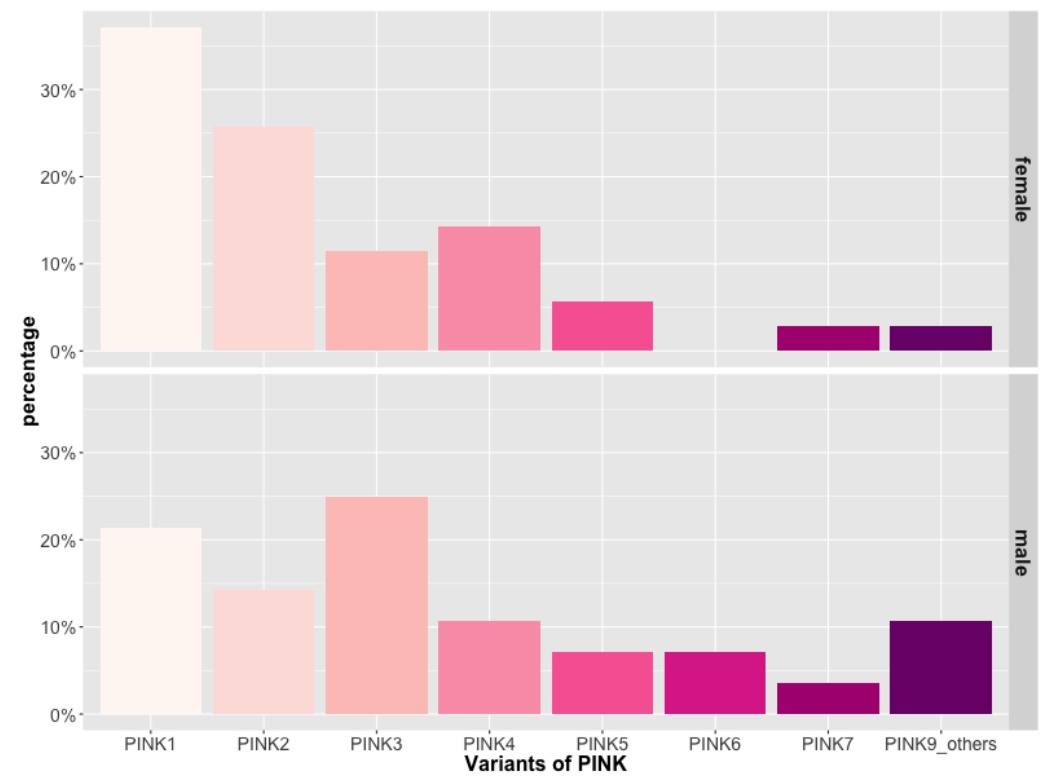

Figure 5.58: Percentage distribution of all PINK variants by gender

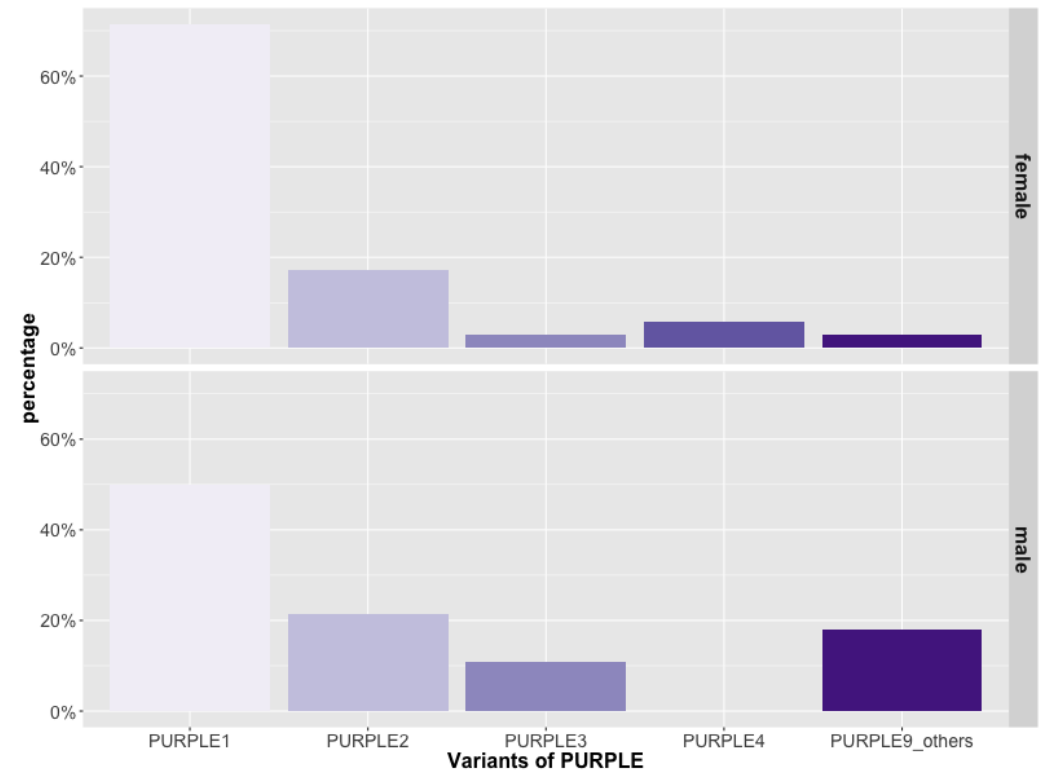

Figure 5.59: Percentage distribution of all PURPLE variants by gender 


\subsubsection{Other social factors}

In Chapter 4, I listed eight social factors to be explored. I also explained the issue of multicollinearity in the datasets. The school factor is related to many other social factors in various ways. For instance, all participants who have deaf parents went to HSD; all those who live in the HKI region had attended CMS; none of the CMS participants work in a deaf related environment. Therefore, trying to predict the use of variants from social factors like language background and work environment would probably display similar patterns to those found when we consider them in relation to signers' schools. Furthermore, the number of signers in the three factor groups examined (i.e., school, age and gender) are relatively evenly distributed, whereas in the other factor groups such as language background, participants are more skewed. For instance, less than $10 \%$ of the sample have deaf parents. This means it would be problematic to use this social factor in statistical calculations when examining individual lexical items. Therefore, it would be more worthwhile and fruitful to explore more social factors in relation to other language behaviours such as the use of compound and handedness in number signs. That is to say, it would be more meaningful to say 'signers who work in a deaf related setting are more likely to use compound signs' than to say 'signers who work in a deaf related setting favour PURPLE2.' Before describing the findings of the use of compound, 'citation form,' and handedness in number signs, I first examine the use of possible homonyms in the next section. 


\subsection{Use of possible homonyms}

There are four pairs of possible homonyms in the dataset: BROWN vs COFFEE, ORANGE vs ORANGE(fruit), PURPLE vs GRAPE, and RED vs HUNGHOM(local place name). As shown in Figure 5.60, many participants use different signs to distinguish BROWN/COFFEE and PURPLE/GRAPE (the white area of the bars). On the other hand, over three-fourths do not separate RED/HUNG-HOM and more than half of the signers use the same sign for the colour and the fruit ORANGE. The high percentage of using the same form for RED and HUNG-HOM may be due to the very distinct meaning of the two concepts and the relatively low occurrence of the local place Hung Hom in everyday discourse. So they are unlikely to occur in contexts where there is genuine potential for confusion.

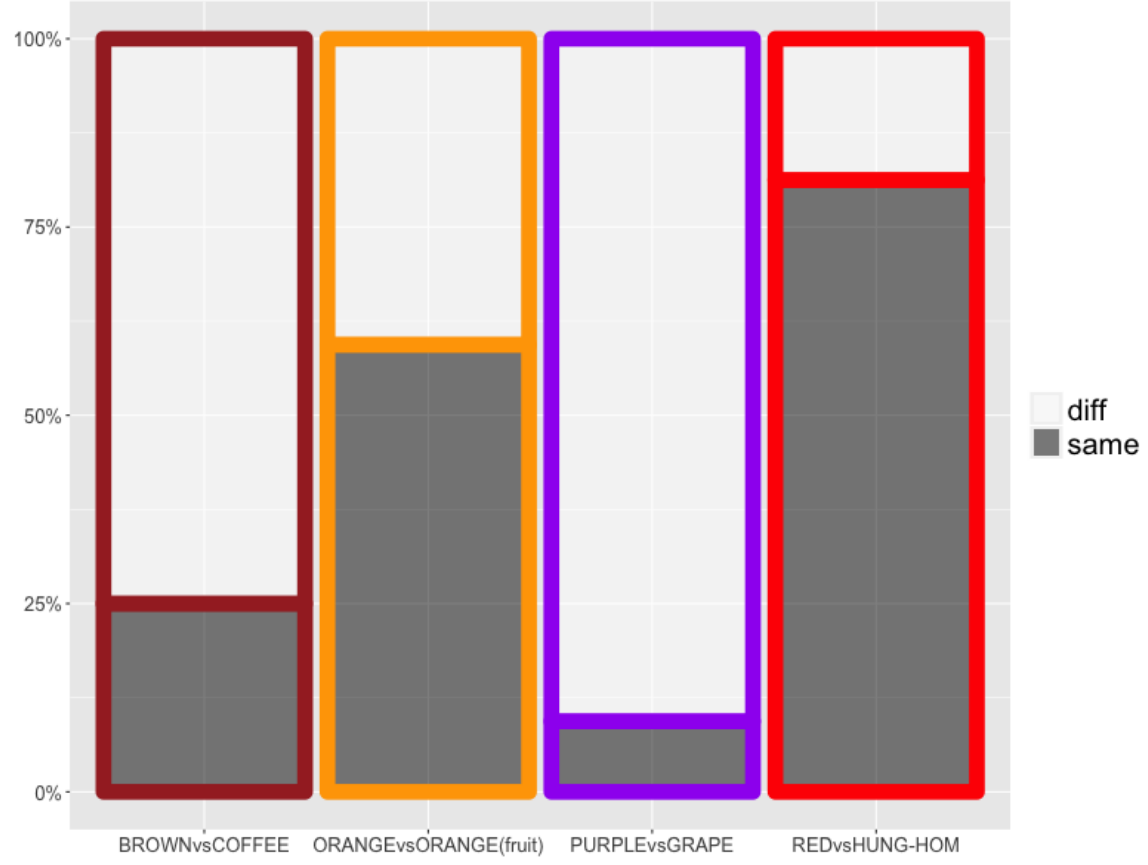

Figure 5.60: Use of homonyms across different pairs 
Social factors seem to have no effect on the use of these four pairs of possible homonyms. Figure 5.61 illustrates that signers from different schools, different age groups, or of different gender demonstrate no significant differences in terms of overall homonym usage. There was no significant difference in factors affecting each of the four pairs of signs. Similar results are found for other social factors such as language background, education, and work environment.

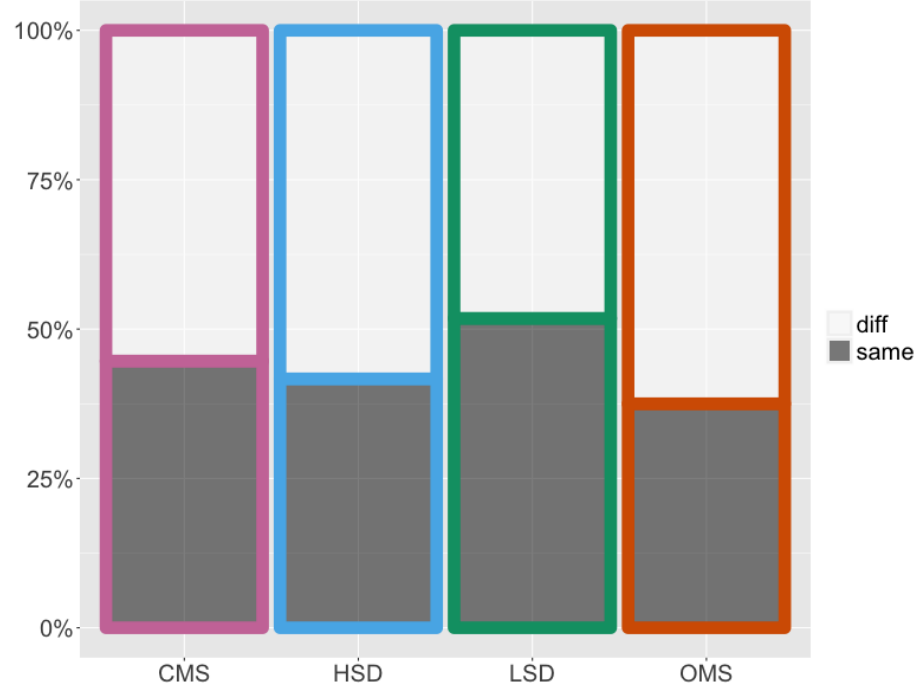

(a) School

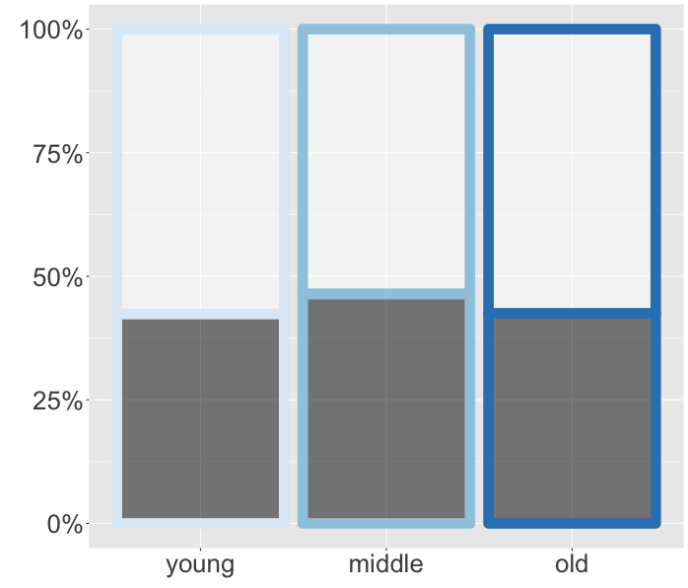

(b) Age

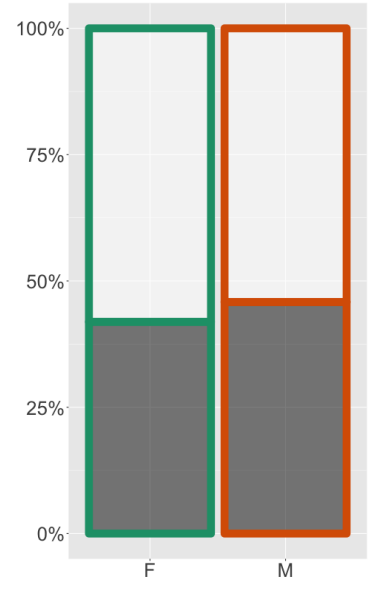

(c) Gender

Figure 5.61: Use of homonyms by social factors: school, age and gender 


\subsection{Compound signs}

To investigate whether older signers produced more compound signs than younger signers (as observed in a preliminary investigation of colour signs mentioned in Chapter 4), a total of 1,345 tokens of the twenty-one concepts that exhibited both simplex and compound forms were coded for analysis. Among these 1,345 tokens, $29.14 \%$ are realised as compound signs. The linguistic factor coded was semantic domain and the social factors included were school, age, gender, education, and work environment. These all demonstrated relatively high importance in a random forest analysis and had shown significance in the initial modeling using $R \cdot{ }^{13}$ Since there is a multicollinearity issue in the dataset, age was included as a continuous variable in the multivariate analysis in Rbrul. Initial analysis showed interactions between other social factors, so they were combined as an independent predictor in the final analysis. Lexical item was included as a random effect.

Table 5.23 shows the results of the Rbrul analysis. The application value is compound (Tagliamonte, 2006). Factor weights above 0.5 generally favour compound, and the closer to 1 , the stronger the effect. The range at the bottom of each factor group is the difference between the factor that favours compound the most and the one that favours it the least within the group. The higher the range value, the stronger the effect. Four social factors, school, gender, education, and work environment interact with each other to form a very significant factor group. In general, those

\footnotetext{
${ }^{13}$ The same method was applied to the datasets of 'citation form' and number handedness in the next two sections. See explanation in Chapter 4.
} 
who went to CMS+, LSD, and OMS+ prefer compound signs. ${ }^{14}$ Over half (55.6\%) of the HSD+ participants favour compounds, the others who disfavour compounds are men in non-deaf workplaces. Most VSD+ signers disfavour compounds, except the women in deaf workplaces.

Age was analyzed as a continuous factor. The result shows that the older signers are more likely to use compound signs, with a 0.36 increase in log odds for every 10-year increment in age. When comparing the use of compound by different age groups, the older group produced more compound signs than the other two groups (see Figure 5.62). This echoes the findings of a preliminary investigation of colour signs mentioned in Chapter 4 (see section 4.5.1.1 on page 92).

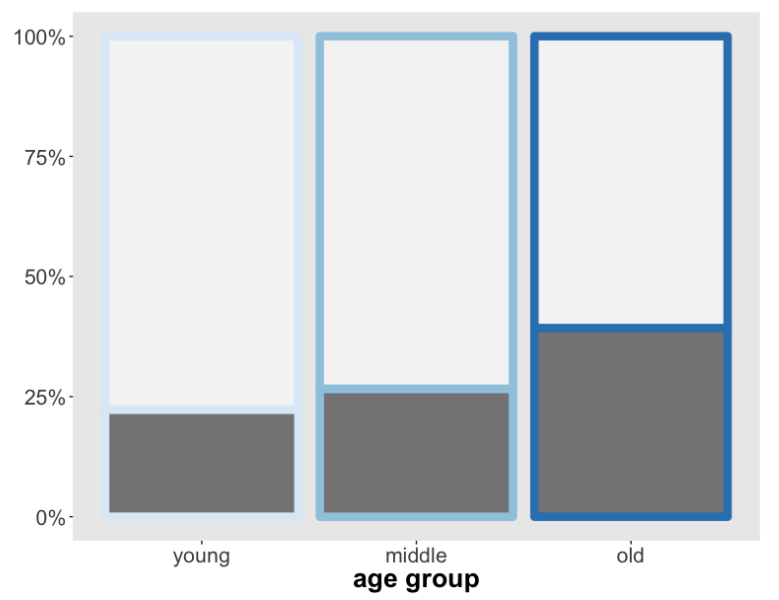

Figure 5.62: Frequency of compound signs by age groups

\footnotetext{
${ }^{14}$ As described in Chapter 4, some respondents went to two schools. The abbreviation CMS+ refers to the signers who went to CMS only and those who went to CMS and another deaf school; OMS+ refers to the signers who went to OMS only and those who went to OMS and another deaf school; and so on.
} 


\begin{tabular}{lrrrr}
\hline Application value: compound & & & & \\
Overall proportion: 0.291 & & & & \\
Deviance: 980.001 & & & & \\
Random (lexical item) Standard deviation: 1.222 & & & & \\
\hline & Log odds & Weight & $\%$ & N \\
\hline School x Education x Gender x Work (p<0.0001) & & & & \\
OMS+.LowEdu.Male.Non-deafWork & 1.459 & 0.811 & 60 & 80 \\
LSD.Average/aboveEdu.Female.Non-deafWork & 0.636 & 0.654 & 37 & 82 \\
HSD+.Average/aboveEdu.Female.DeafWork & 0.615 & 0.649 & 30 & 83 \\
LSD.Average/aboveEdu.Male.Non-deafWork & 0.589 & 0.643 & 37 & 101 \\
OMS+.LowEdu.Female.Non-deafWork & 0.434 & 0.607 & 42 & 104 \\
CMS+.Average/aboveEdu.Male.Non-deafWork & 0.429 & 0.606 & 33 & 63 \\
LSD.Average/aboveEdu.Female.DeafWork & 0.384 & 0.595 & 30 & 105 \\
HSD+.Average/aboveEdu.Male.DeafWork & 0.360 & 0.589 & 27 & 63 \\
HSD+.Average/aboveEdu.Female.Non-deafWork & 0.261 & 0.565 & 32 & 62 \\
VSD+.Average/aboveEdu.Female.DeafWork & 0.237 & 0.559 & 27 & 63 \\
CMS+.Average/aboveEdu.Female.Non-deafWork & 0.227 & 0.557 & 37 & 41 \\
VSD+.Average/aboveEdu.Female.Non-deafWork & -0.616 & 0.351 & 20 & 186 \\
OMS+.Average/aboveEdu.Male.DeafWork & -0.797 & 0.311 & 29 & 21 \\
VSD+.Average/aboveEdu.Male.Non-deafWork & -1.187 & 0.234 & 16 & 125 \\
HSD+.LowEdu.Male.Non-deafWork & -1.348 & 0.206 & 21 & 42 \\
HSD+.Average/aboveEdu.Male.Non-deafWork & -1.684 & 0.157 & 11 & 124 \\
Range & & 65 & & \\
\hline Age (p<0.001) & & & & \\
+10 & & & & \\
\hline Semantic domain (p<0.01) & -0.016 & 0.496 & 25 & 194 \\
Kinship & -0.630 & 0.347 & 17 & 445 \\
Food & -1.633 & 0.163 & 6 & 65 \\
Colour & & 78 & & \\
Country/region & & & & \\
Local place & & & & \\
Range & & & & \\
\hline Total(N) & & & & \\
\hline & & & & \\
\hline
\end{tabular}

Table 5.23: Results of Rbrul analysis of significant factor groups for compound signs

As Table 5.23 shows, the proportion of compound use is not directly aligned with the factor weight in this combined factor group. This is shown by the percentage values which do not decrease in the same way the weightings do. This may indicate that there is an interaction between this factor group and age. With regard to the linguistic factor, semantic domain is found to be significant with a range value of 78 . Kinship 
terms favour compound forms, whereas all other semantic domains (food, colour, country/region, and local place) disfavour them.

Since the dataset is complex and the social factors seem to be interacting with each other, I also constructed conditional inference trees to explore the relationship between the social factors. Figure 5.63 illustrates the tree in which age is modelled as a continuous factor. We can see from the tree that signers from different schools have a different pattern of compound use. Those who went to CMS+, LSD, or OMS+ behave similarly, with a higher proportion of compound use as opposed to those had studied in HSD+ or VSD+. For the group that use more compounds (i.e., CMS+, LSD, and OMS+), participants with lower educational level tend to use even more. Half of the tokens produced by less educated signers are compounds, while about one-third of the tokens are compounds for those with average or above educational level. On the other hand, work environment and age constrain the compound choice of HSD+ and VSD+ participants. For those who work in a deaf related setting, near $30 \%$ of their tokens are compounds. This is a similar proportion to those who do not work in a deaf related setting, but are over 40 years of age. On the contrary, younger HSD+ and VSD+ signers (aged 40 or below) working in non-deaf environment produced fewer compounds (slightly over 10\%). 


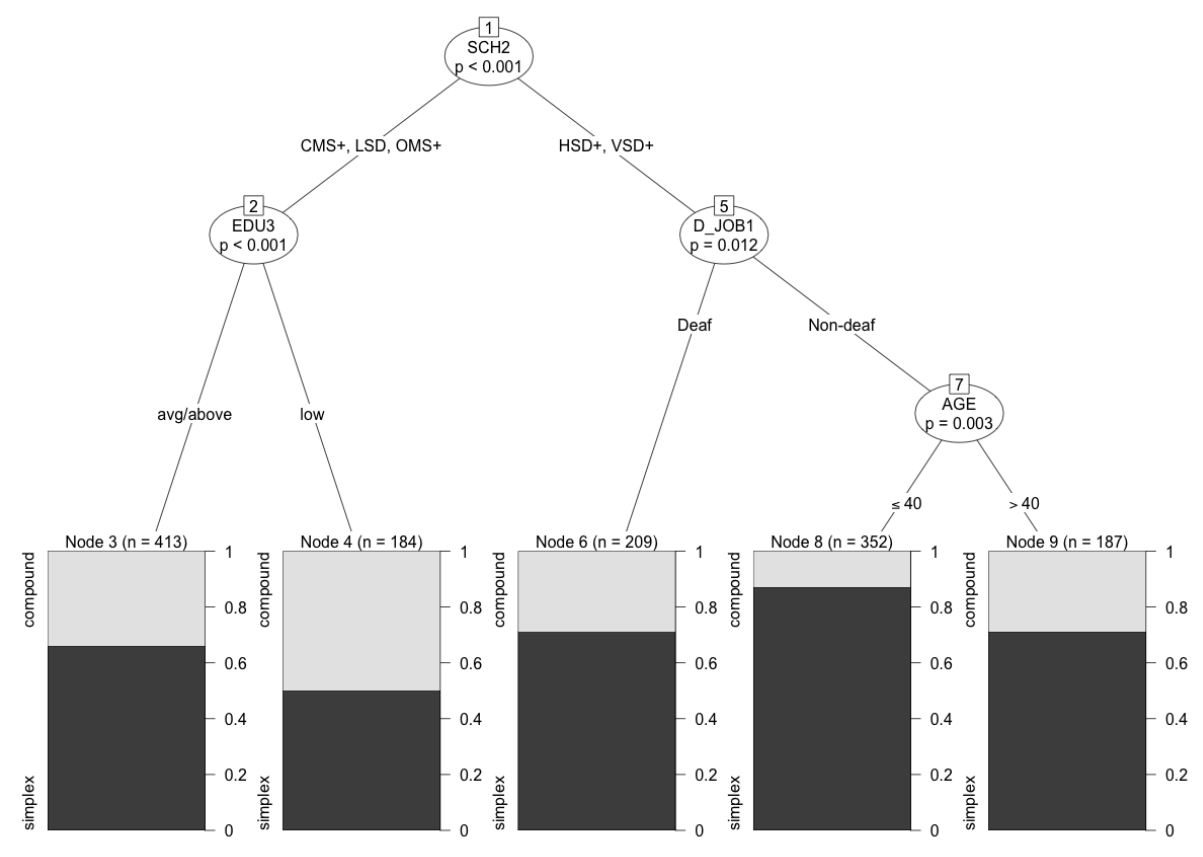

Figure 5.63: Conditional inference tree for compound signs (age as continuous)

Interestingly, when I used age group as a factor instead of age as a continuous variable, the tree reveals a gender difference. As shown in Figure 5.64, the left part of the tree is the same as the one in Figure 5.63. At the node where age is replaced by age group, a new branch indicating gender difference is created for the younger and the middle-aged groups. In this non-older group, women use significantly more compounds than men, $20 \%$ for the former and less than $10 \%$ for the latter $(\mathrm{p}=0.012)$. 


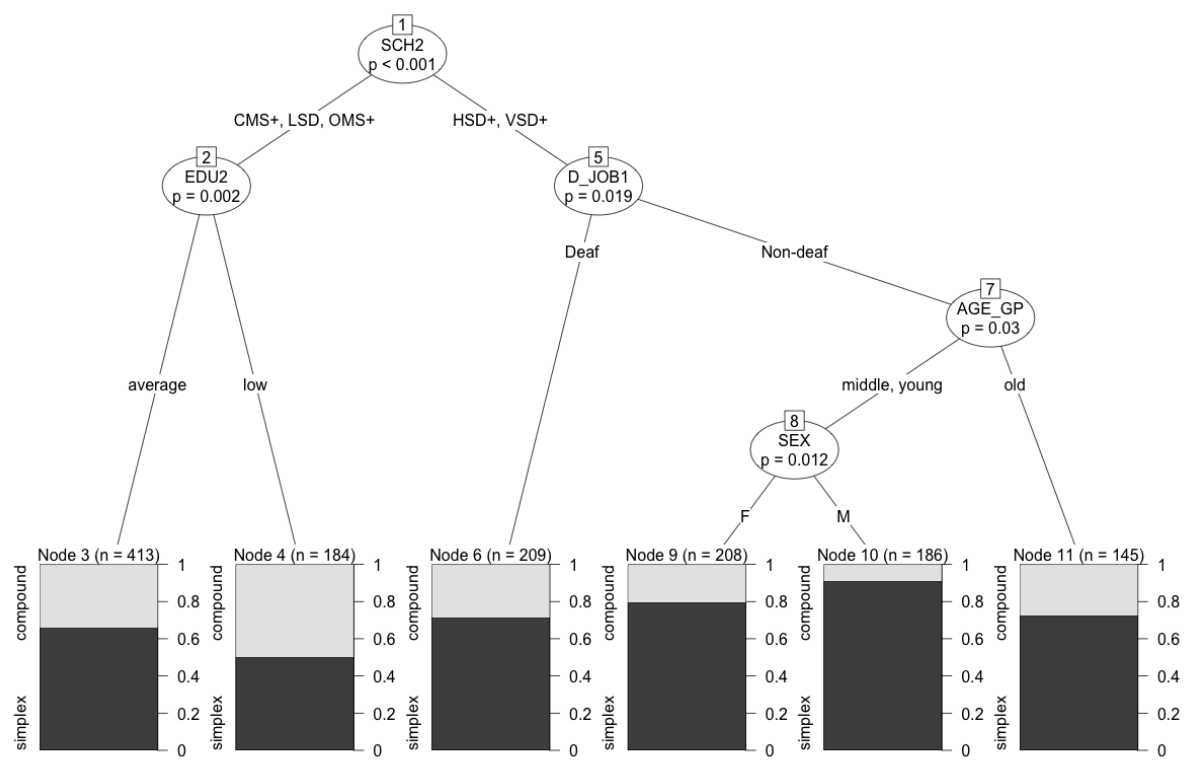

Figure 5.64: Conditional inference tree for compound signs (age as group)

Overall, the conditional inference trees support the results of the multivariate analysis and they show how the social factors interact from a different angle. In short, school is a prominent indicator of compound use. Those who had studied in CMS+, LSD, or OMS+ tend to use more compounds, particularly those who had lower educational attainment. While those who went to HSD+ or VSD+ prefer simplex over compound signs, signers aged over 40 from a non-deaf workplace and those from deaf workplace produced more compounds. Furthermore, female signers in the younger or the middle-aged group (i.e., aged below 50) use more compounds than their male counterparts.

The relative preference of compound use by those less educated and those from a deaf workplace may indicate that they want to make themselves more clear by using compounds. This may be especially true when they know there is more than one variant for a given concept. Indeed, in 
some cases, the compound signs are compounds of two known variants. Overall, the signers least likely to use compound signs are men under 50 working in non-deaf environments (as we will see in subsequent sections, younger men are associated with use of more innovative variants for some other variables too).

\section{7 'Citation form'}

Based on the 'citation form' defined in Section 4.5.1.2, 1,298 tokens of twenty concepts were coded for analysis. Fifty-eight percent of the forms in the dataset were 'citation forms.' Like the analysis of compound signs, semantic domain was the only linguistic factor coded and the five social factor groups included were the same as the compound analysis: school, age, gender, education, and work environment. Again, age was included as a continuous variable to alleviate the problem of multicollinearity during the model-building process and lexical item was treated as random effect.

The result of the multivariate analysis is shown in Table 5.24. It is obvious that all those who work in a deaf related setting favour the use of 'citation forms.' Those with higher educational level tend to use more 'citation forms' than those whose educational attainment is average or below. Female signers, in general, prefer 'citation forms' more than male signers do. Older signers also favour 'citation forms,' with a 0.21 increase in log odds for every 10-year increment in age. Among the four schools, CMS+ is more likely to use 'non-citation forms' than others. 


\begin{tabular}{|c|c|c|c|c|}
\hline \multicolumn{5}{|l|}{$\begin{array}{l}\text { Application value: 'citation form' } \\
\text { Overall proportion: } 0.575 \\
\text { Deviance: } 1289.34 \\
\text { Random (lexical item) Standard deviation: } 1.043\end{array}$} \\
\hline & Log odds & Weight & $\%$ & $\mathrm{~N}$ \\
\hline \multicolumn{5}{|l|}{ School $\mathbf{x}$ Education $\mathbf{x}$ Gender $\mathbf{x}$ Work $(\mathrm{p}<0.0001)$} \\
\hline HSD+.HighEdu.Female.DeafWork & 1.165 & 0.762 & 77 & 60 \\
\hline HSD+.Average/belowEdu.Female.DeafWork & 1.005 & 0.732 & 75 & 80 \\
\hline HSD+.HighEdu.Male.DeafWork & 0.760 & 0.681 & 70 & 40 \\
\hline HSD+.Average/belowEdu.Male.DeafWork & 0.434 & 0.607 & 65 & 20 \\
\hline LSD.Average/belowEdu.Female.DeafWork & 0.403 & 0.599 & 66 & 100 \\
\hline OMS+.Average/belowEdu.Male.DeafWork & 0.342 & 0.585 & 75 & 20 \\
\hline HSD+.Average/belowEdu.Female.Non-deafWork & 0.228 & 0.557 & 68 & 99 \\
\hline HSD+.HighEdu.Male.Non-deafWork & 0.201 & 0.550 & 68 & 60 \\
\hline LSD.Average/belowEdu.Female.Non-deafWork & 0.125 & 0.531 & 64 & 80 \\
\hline LSD.Average/belowEdu.Male.Non-deafWork & -0.384 & 0.405 & 55 & 100 \\
\hline OMS+.Average/belowEdu.Male.Non-deafWork & -0.389 & 0.404 & 63 & 79 \\
\hline OMS+.Average/belowEdu.Female.Non-deafWork & -0.470 & 0.385 & 60 & 100 \\
\hline CMS+.Average/belowEdu.Female.Non-deafWork & -0.587 & 0.357 & 51 & 180 \\
\hline CMS+.Average/belowEdu.Male.Non-deafWork & -1.171 & 0.237 & 39 & 140 \\
\hline HSD+.Average/belowEdu.Male.Non-deafWork & -1.661 & 0.160 & 34 & 140 \\
\hline Range & & 60 & & \\
\hline \multicolumn{5}{|l|}{ Age $(p<0.01)$} \\
\hline+10 & 0.210 & & & \\
\hline \multicolumn{5}{|l|}{ Semantic domain $(p<0.05)$} \\
\hline Kinship & 1.318 & 0.789 & 76 & 455 \\
\hline Number & 0.220 & 0.555 & 55 & 389 \\
\hline Colour & -0.420 & 0.397 & 44 & 324 \\
\hline Country/region & -1.118 & 0.246 & 32 & 130 \\
\hline Range & & 54 & & \\
\hline Total(N) & & & & 1298 \\
\hline
\end{tabular}

Table 5.24: Results of Rbrul analysis of significant factor groups for 'citation form'

In addition, the conditional inference tree for 'citation form' reveals a similar pattern. Figure 5.65 shows the tree with age as a continuous variable. Work environment is the strongest predictor of 'citation form' usage, with deaf related work highly favouring 'citation forms' (about 70\% 'citation forms' used). Among those who work in a non-deaf environment, participants aged over 40 prefer 'citation forms,' while those aged 40 or below are more mixed. For women aged 40 or under, those who 
went to HSD+ or LSD use significantly more 'citation forms' than those who went to CMS+. However, for men of the same age range, educational level predicts their 'citation form' preference. Men aged 40 or below with higher education strongly favour 'citation forms' (over 70\% 'citation forms' produced), whereas those with average or below education disfavour it (around 30\% 'citation forms' made).

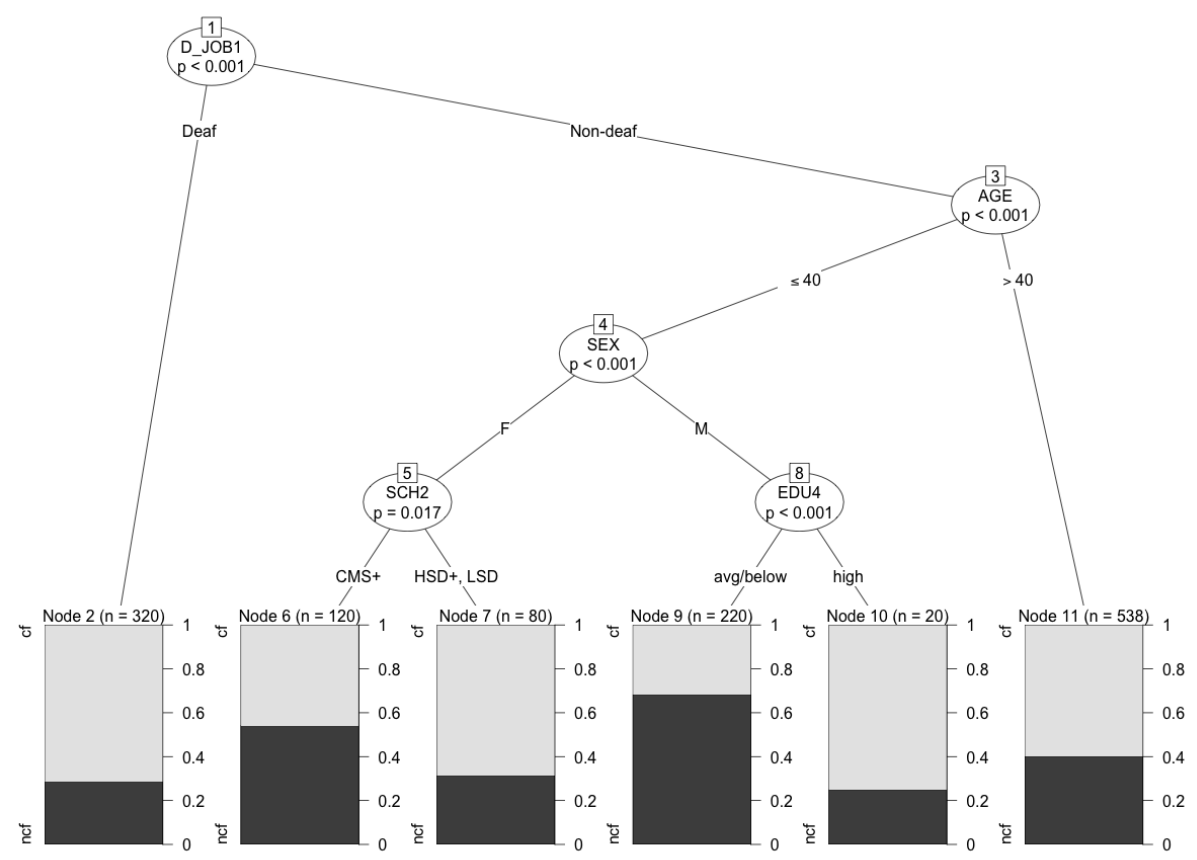

Figure 5.65: Conditional inference tree for 'citation form' (age as continuous) (cf=citation form; ncf=non-citation form)

On the other hand, when age was included as discrete variable, a slightly different tree was constructed. As we can see in Figure 5.66, deaf-related work is still the strongest predictor. The next prominent factor is gender, with women generally favouring 'citation forms.' For men, highly educated younger or middle-aged signers have a strong preference for 'citation forms.' Older male signers (aged 50 or over) also prefer 'citation 
forms' (nearly $60 \%$ ). While younger or middle-aged men having average or below educational level tend to use less 'citation forms,' those who went to CMS+ or HSD+ use fewer than those who studied in LSD $(p=0.009)$.

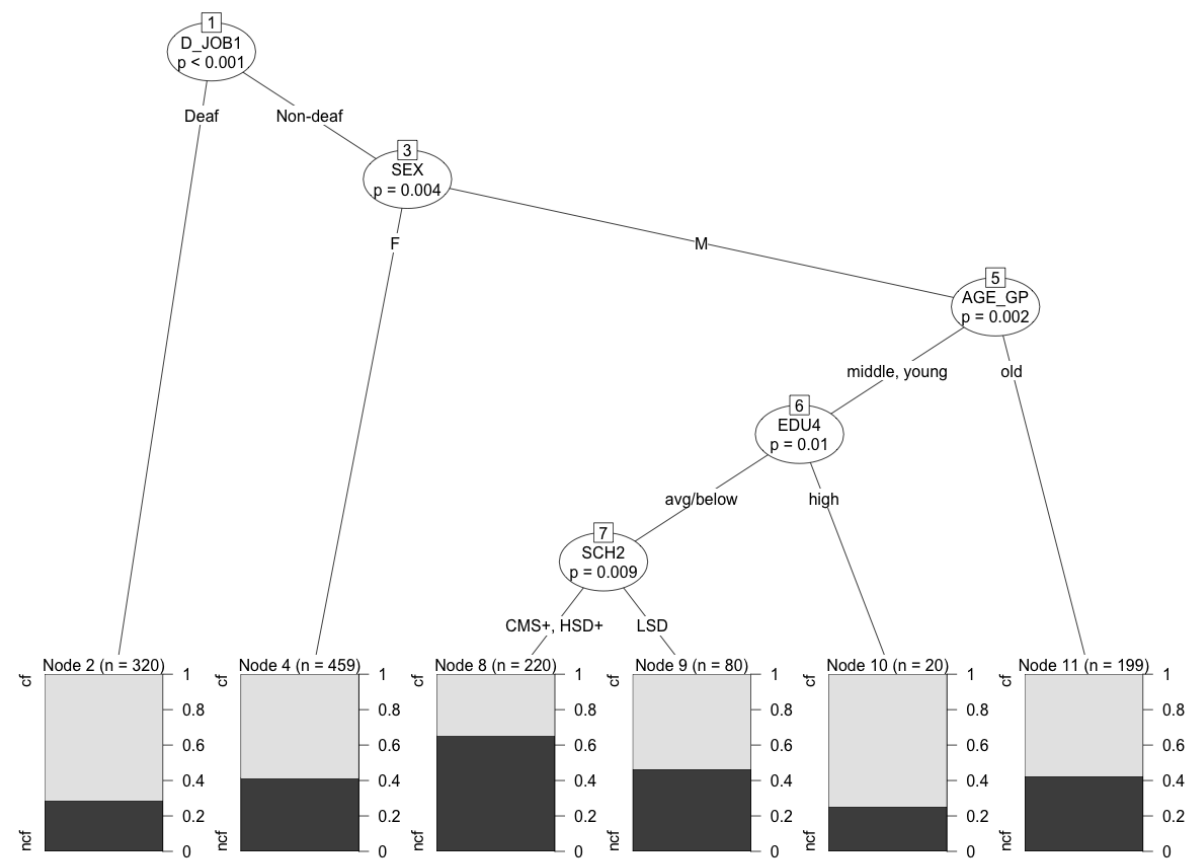

Figure 5.66: Conditional inference tree for 'citation form' (age as group)(cf=citation form; ncf=non-citation form)

In a nutshell, work environment is the strongest constraint on the use of 'citation form' and 'non-citation form.' As explained in Chapter 4, those who work in a deaf related setting are very likely to perform the role of language model or language adviser. Therefore, they may be expected to produce 'citation forms.' Older signers, women, and highly educated men also tend to use more 'citation forms' than their counterparts. It is possible that the 'citation form' for some of these concepts is viewed as the 'proper signs' (i.e., are above the level of conscious awareness) in the deaf community, but attitudes like this were not directly tested. In addition, the school 
differentiation may reflect the fact that the 'citation forms' in the five HKSL publications are representative of certain schools. In particular, the CMS variants are least used in those imprints and we see in both Figures 5.65 and 5.66 that CMS+ signers are always modelled as disfavouring 'citation forms.'

\subsection{Numbers: one-handed versus two-handed}

As mentioned earlier, number signs above ten can be produced with either one or two hands in HKSL. Fifteen number signs in the lexical data were coded for handedness. There were 975 tokens in this dataset, of which $28.6 \%$ are two-handed signs. In order to investigate what kinds of numbers are more likely to be signed with two hands, these numbers were classified into four classes: tens, twenties, twenty/thirty, and hundred. This number class was the only linguistic factor included and the social factors were the same as the analyses of compound signs and 'citation form': school, age, gender, education, and work environment. Age was included as a continuous variable. Fixed-effects modeling was employed (see explanation in Chapter 4). The significant factors of the Rbrul analysis are shown in Table 5.25 . 


\begin{tabular}{lrrrr}
\hline $\begin{array}{l}\text { Application value: two-handed } \\
\text { Input probability: 0.26 }\end{array}$ & & & & \\
Deviance: 569.815 & & & & \\
\hline & & & & \\
\hline Number class (p<0.0001) & & & & \\
Hundred & 3.034 & 0.954 & 69 & 65 \\
Tens & 1.744 & 0.851 & 52 & 390 \\
Twenties & -2.218 & 0.098 & 6 & 390 \\
Twenty \& thirty & -2.559 & 0.072 & 5 & 130 \\
Range & & & & 88 \\
\hline School x Education x Gender (p<0.0001) & & & & \\
CMS+.Average/aboveEdu.Male & & & \\
LSD.Average/aboveEdu.Male & 2.760 & 0.940 & 64 & 45 \\
CMS+.Average/aboveEdu.Female & 1.458 & 0.811 & 49 & 75 \\
HSD.LowEdu.Male & 1.188 & 0.766 & 47 & 30 \\
OMS+.LowEdu.Male & 1.188 & 0.766 & 47 & 15 \\
OMS+.LowEdu.Female & 1.052 & 0.741 & 45 & 75 \\
LSD.Average/aboveEdu.Female & 0.916 & 0.714 & 44 & 75 \\
OMS+.Average/aboveEdu.Male & 0.663 & 0.660 & 42 & 135 \\
VSD+.Average/aboveEdu.Male & 0.518 & 0.627 & 40 & 15 \\
VSD+.Average/aboveEdu.Female & -0.746 & 0.322 & 26 & 90 \\
HSD.Average/aboveEdu.Male & -1.688 & 0.156 & 15 & 180 \\
HSD.Average/aboveEdu.Female & -2.414 & 0.082 & 9 & 135 \\
Range & -4.894 & 0.007 & 1 & 105 \\
\hline Total(N) & & & & 93 \\
\hline & & & & 975 \\
\hline & & &
\end{tabular}

Table 5.25: Results of Rbrul analysis of significant factor groups for two-handed number signs

We can see in Table 5.25 that number class is a very significant factor group (range is 88). Hundred and tens strongly favour the use of two hands (factor weights are 0.954 and 0.851 respectively), while twenty to thirty tend to be produced with one hand (both factor weights are below 0.1). With regard to social factors, school, education, and gender interact with each other to form the strongest factor group (range is 93). Those who went to HSD or VSD+ strongly favour one-handed number signs, but all others prefer the two-handed form. Participants who have lower educational level tend to use more two-handed number signs. In particular, we can see this effect when we compare the man who went to HSD with a fac- 
tor weight of 0.766 , as opposed to his better educated counterparts whose factor weight is 0.082 . In general, men are more likely to use two-handed forms than women. This pattern is consistent when we compare male and female signers from the same school and with the same educational attainment.

Although work environment is not a significant factor in the Rbrul analysis, it seems to play a role in constraining the use of handedness in number signs, especially for those who went to HSD and VSD+. As shown in Figures 5.67 and 5.68, for HSD and VSD+, those who work in deaf related settings almost never produced two-handed number signs. For those who work in a non-deaf company, education and age are additional factors. When age is treated as a continuous variable (see Figure 5.67), participants with lower education tend to use more two-handed forms, nearly $50 \%$. Among those who have average or above educational level, VSD+ signers appear to use more two-handed forms (slightly over 20\%) than HSD (around 10\%). When age is treated as a discrete variable (see Figure 5.68), age is the constraining factor and education is no longer a factor. The younger and the older groups use more two-handed forms (over $20 \%$ ) than the middle-aged group (around $10 \%$ ).

However, when we look at the two trees more closely, we can see that the HSD bar at node 7 in Figure 5.67 (age as continuous) may refer to the same group of people as the middle-aged bar at node 6 in Figure 5.68 (age as group). Checking with the participants information confirmed this observation. This means that all the HSD signers from non-deaf workplace are middle-aged and have average or above educational level. Also, in Figure 5.67, all the VSD+ signers at node 8 are young and all the less 
educated signers at node 9 are older HSD signers. This explains why the younger and older groups behave similarly whereas the middle-aged group shows a different pattern when age group is used in constructing the tree.

To conclude, number class and school are the strongest predictors of handedness in number signs. Signs for hundred and tens highly favour two-handed forms, whereas twenty to thirty highly favour one-handed forms. CMS+, LSD, and OMS+ prefer two-handed number signs, but HSD and VSD+ prefer one-handed. Both the results of an Rbrul analysis and the conditional inference trees indicate the prominent role of school in the choice of number handedness. Gender is found to be one of the interacting factors in the regression analysis, but not in the conditional inference trees; work environment is found to be significant in the latter, but not in the former. Education is also a confounding factor. On the whole, men use more two-handed forms than women, but less educated signers as a group are more likely to use two-handed number signs. As revealed in the conditional inference trees, work environment constrains the variant choice of the HSD and VSD+ signers. Although both HSD and VSD+ signers favour one-handed number signs, the former produced a significantly higher proportion of one-handed forms than the latter. 


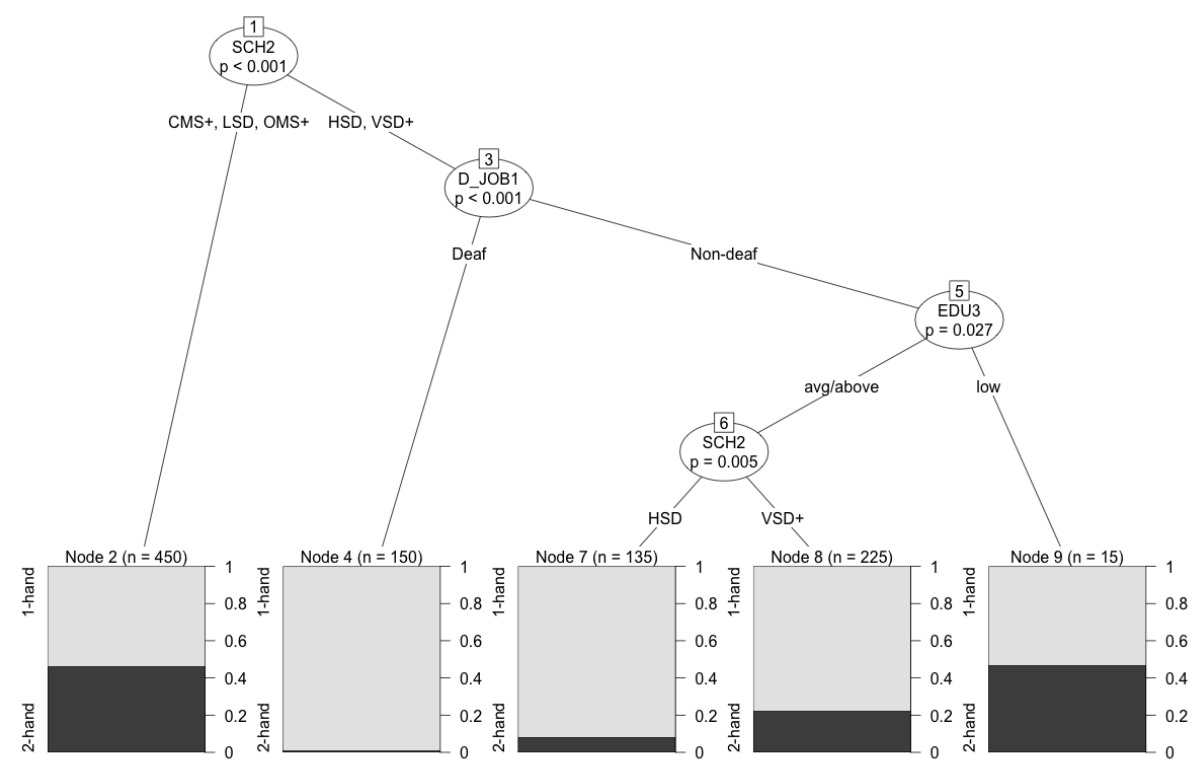

Figure 5.67: Conditional inference tree for handedness in number signs (age as continuous)

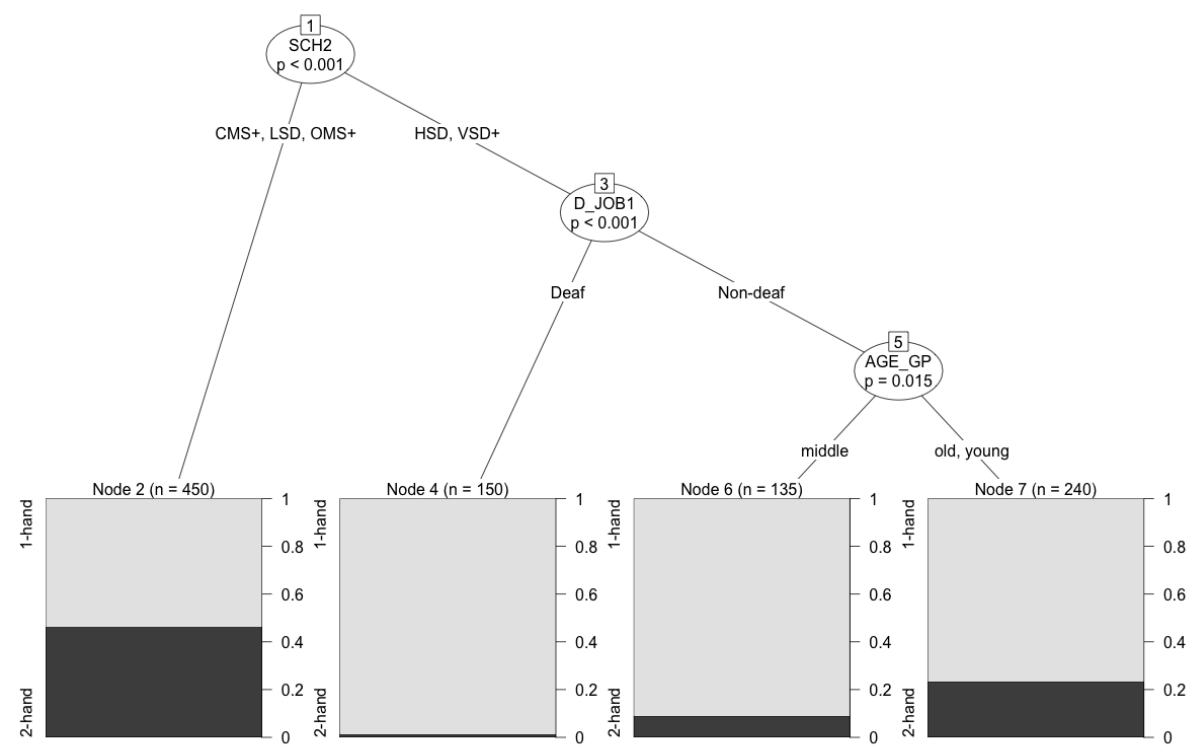

Figure 5.68: Conditional inference tree for handedness in number signs (age as group) 


\subsection{Language change in progress?}

At the beginning of this chapter in Section 5.2, I reported that there is no variation found in the sign YELLOW. Informants from all age groups gave me the same 'modern form,' and none of them produced the 'old form.' This seems to signal a completed change for YELLOW such that most if not all members of the HKSL community would use the 'modern form' to refer to the colour yellow. The 'old form' may only appear when using someone's name sign which includes this form.

Apart from YELLOW, all other lexical items display different degree of variation. Does this variation offer any evidence of language change in progress in the lexical items examined? What types of phonological processes are involved? What is the role of social factors with regard to the change observed? These questions will be answered based on the findings presented in the section about age-differentiated variants (Section 5.4.2) and the analysis of the three data subsets, compound signs, 'citation form,' and handedness in number signs.

As stated earlier, some old variants are used only by a small number of middle-aged or older signers. These old variants are gradually being replaced by the new variants and will probably vanish in the near future. The change of these three lexical items, BLACK, PURPLE, and AUSTRALIA, may be completed or nearly completed. Interestingly, the new variants are all separate distinct variants, not phonologically related variants. Further, when we compare the handshapes of the three old variants with those of the subsequent ones, we notice that the latter (the most frequent variants of each concept) are made up of more marked handshapes than the old 
disappearing variants, which use unmarked handshapes. This seems to be in contrast to the role that markedness usually plays in language variation and change (Trudgill, 1986).

The shift from an early form used more by the older signers to a distinct variant used more by the younger signers is also observed in PINK and INDIA. In PINK, the early variants are all compounds whereas the modern form is a simplex sign with a marked handshape. For AUSTRALIA and TAIWAN, there is a tendency to adopt the country/region's own local sign by the middle-aged and the younger groups. However, this disposition may be weakened by the potential resistance from the signers who are becoming proud of their own language as explained in the case of ITALY in Section 5.4.2. Having said that, there is another view saying that adopting other countries/regions' own signs is an act of paying respect to the sign language of other nations. In short, the course of this change (or potential change) needs to be watched.

Reviewing the findings of the previous studies (Chen \& Tai, 2009; Frishberg, 1975; Sze, Chu, et al., 2013; Woll, 1987), the following changes have been observed in signed languages:

- two-handed signs become one-handed

- location has become more centralized

- location at the face has been lowered

- movement has changed

- there is assimilation of the two parts in a compound

- there is deletion of one part in a compound 
These changes are also found in the lexical items examined.

The change from a two-handed to a one-handed form is found in one of the variants of ORANGE. ORANGE2b is the two-handed version used only by a few older signers; ORANGE2a is the one-handed form that is used by all three age groups, especially by the younger signers (see Figure 5.14 on page 140 for sign illustrations). Additionally, the one-handed variant, TAIWAN2b, is evolved from the compound in which the second part is two-handed (I will say more on this shortly).

Concerning the parameters, centralization in location is observed in BEIJING1. In BEIJING1a, the 2-handshape is moving horizontally across the upper chest, making the ending location closer to the beginning location. Hence, it looks more centralized when compared with the original form, BEIJING1b, which ends in a lower location (see Figures 5.32a and 5.32b on page 152 for sign illustrations). While the older group favours the original form (BEIJING1b), the middle-aged and the younger groups prefer the more centralized form (BEIJING1a). The middle-aged produced more of the new forms which may suggest that they are the ones who have led the change.

For signs articulated at the face such as RED, there is a lowered variant REDb (see Table 5.9 on page 173). This lowered form is made largely by the younger group. The middle-aged and the older groups favour the original form (REDa) which touches the lower lip. Apart from that, the younger group has initiated the movement change in the numbers in the twenties including TWENTY-ONE, TWENTY-THREE, and TWENTY-FIVE (see Table 5.13 on page 176). Moreover, the younger signers have introduced a palm orientation change in GREEN (see Table 5.10 on page 173). 
With regard to the compounds, two types of diachronic change have been noted in the previous studies (Frishberg, 1975; Woll, 1987). One is the assimilation of the two parts, making the form more like a single sign rather than a compound of two separate signs; the other is the deletion of one part of a compound, either the first or the second part, and the retention of the other part. Both forms are observed in TAIWAN. In TAIWAN2b, the non-dominant hand in the second part of the compound is deleted. When the index finger moves away from the face, it closes with the thumb and finishes the sign with the fingers (palm orientation) facing the signer. It is no longer possible to divide the sign into two parts as the movement is shortened and smoothened, which makes it look like one single sign (see Figure 5.54a on page 179). In TAIWAN3, the first part of the compound is deleted and the second part is retained (see Figure 5.54b on page 179). Yet, both simplified forms are merely minor variants. This may imply an early change of the original sign.

On the contrary, in BEIJING, a compound form has been created by combining an early variant and a modern variant. This new form, BEIJING3, has apparently been initiated by the older signers. It may reflect an ambivalence towards the upsurge of new variants (both a separate variant and a derived variant). In fact, compounding two variants to form a new sign appears in a number of items and it is not solely produced by the older signers. This is probably one of the strategies that HKSL signers use to deal with the variation around them. By compounding two or three variants, a form may be more comprehensible to a wider audience.

Although the use of compounds is not confined to the older group, the older generation did produce more compounds than the younger and 
the middle-aged generations as revealed in the analysis of the compound dataset. From the results of the conditional inference trees, we can see that this age effect is especially relevant to the signers who had attended HSD or VSD, and who do not work in a deaf workplace (see Figure 5.63 on page 200 and Figure 5.64 on page 201). Therefore, the appearance of a change in progress should be considered with caution. Furthermore, even though it may be the case that the change is happening within the HSD and VSD signers, the fact that there seem to be more signers entering deaf workplaces may well retard such a change.

Similarly, in the case of 'citation forms,' although age appears to be a prominent factor in the multivariate analysis, the results of the conditional inference trees reveal that the age effect is associated with the signers working in non-deaf settings. Even within those aged 40 and below (or the non-older group), the extent to which 'citation form' is used is further constrained by gender, school, and one's educational level (see Figure 5.65 on page 204 and Figure 5.66 on page 205). Thus, this age effect may not signal a language change in progress in which the younger generation as a whole are using fewer 'citation forms.' On the contrary, the younger signers may be inclined to adopt more 'citation forms,' as work environment is a strong effect and we have reason to believe there will be more signers (especially the younger ones) working in deaf workplaces in the future.

Regarding the handedness in the number signs above ten, the results show that age is not a factor constraining the choice between one-handed or two-handed number signs. Apart from the social factors: school, education, and gender, number class is also a highly significant factor. While hundred and tens strongly favour the two-handed form, numbers twenty 
to thirty highly favour the one-handed form. In view of the overall high percentage of one-handed forms in the dataset $(71.4 \%)$, I speculate that the change from two-handed to one-handed forms in the numbers twenty to thirty (TWENTY, TWENTY-ONE, TWENTY-TWO, TWENTY-THREE, TWENTYFIVE, TWENTY-SEVEN, TWENTY-EIGHT, and THIRTY in the dataset) may have gone to a further stage than the other numbers (ELEVEN, THIRTEEN, FIFTEEN, SEVENTEEN, EIGHTEEN, and HUNDRED in the dataset). Certainly, more work needs to be done in order to explore this complicated issue.

Nevertheless, the data is obtained through a decontextualized elicitation task, which means the results do not inform us about the complete repertoire of signers in discourse in various contexts, and signers may use different variants according to the audience, context, and situation. The results here should be viewed as an initial step in understanding language change. Any claims or conclusions should be verified by conversational data, ideally from a balanced corpus.

\subsection{Chapter summary}

In this chapter, I have reported the findings of the analysis of the lexical items elicited which covered four semantic domains: colour, kinship term, country/region, and number. Distinct lexical variants are more common than phonologically related subvariants in the items examined. The more frequently used variants tend to have more phonological variants. Some signs which have classifier origins appear to be in the process of lexicalization and exhibit a high degree of variation. Idiosyncratic variation seems to be common. 
With regard to social factors, school is found to be a strong predictor in lexical variant choices. Age differences and gender differences are also found in some lexical items. Nonetheless, social factors seem to have no effect on the use of the four pairs of possible homonyms investigated. In addition to individual lexical items, the use of compound signs, 'citation forms,' and handedness in number signs were also examined. For the use of compounds and 'citation forms,' school, education, gender, and work environment interact with each other to explain the trend. Age is also a significant factor. The older the signer is, the greater the tendency for them to use compounds and 'citation forms.' While numbers over ten can be produced either one-handed or two-handed, signs for hundred and tens favour the latter. Extra-linguistic factors including school, education, and gender are revealed in the Rbrul analysis. Results of condition inference trees suggest work environment and age are also significant constraints operating within the dataset.

It is interesting to see how the five social factors: school, age, gender, education, and work environment, consistently affect the three variables: compound signs, 'citation forms,' and handedness in number signs. Bearing in mind the interrelatedness of the social factors discussed in Chapter 4, the interaction between school, age, education, and work environment may not be so surprising. What is more surprising is that men and women behave differently in the use of compounds, 'citation forms,' and number handedness. The age effect found in the use of compounds and 'citation forms' does not seem to indicate any change.

Finally, there are lexical items that demonstrate change in progress and one of them even shows a completed change. The formational or phono- 
logical changes observed are consistent with those found in earlier studies. For instance, two-handed signs become one-handed, there is location centralization, location lowering, change in movement, and deletion and blending in compounds. In addition, some 'old' signs with an unmarked handshape are being replaced by a 'modern' form with a marked handshape; there is sign exhibiting palm orientation change, and a noncompound sign has become a compound sign.

In the next chapter, I will turn to the first phonological variable: the movement pattern in DEAF and HEARING. I will present the findings of these two items from the lexical data (which I have not reported in this chapter), and discuss the analysis of the variables in the conversation data. 


\section{Chapter 6}

\section{Phonological variation 1: DEAF and HEARING}

\subsection{Overview}

This chapter addresses the first phonological variable, that is, the movement pattern in the signs DEAF and HEARING. Figures 6.1 to 6.5 illustrate the five variants $(\mathrm{HMH}, \mathrm{MMM}, \mathrm{MH}, \mathrm{M}, \mathrm{H})^{1}$ of the two signs. Before presenting the analysis of these two variables from the conversation data, I first report the findings of the two signs from the lexical elicitation task in Section 6.2. In Section 6.3, I begin by providing the overall findings for DEAF and HEARING. In subsections, Sections 6.3.1 and 6.3.2, I describe the results in terms of linguistic factors and social factors respectively. I

${ }^{1}$ See description of the codes in Section 4.5.2.1 on page 102. 
then discuss the results of my analysis in Section 6.4. Comparison with the results of the ASL study is given in Section 6.4.1. The issue of language change and some potential implications are discussed in Sections 6.4.2 and 6.4.3 respectively. Lastly, Section 6.5 is the chapter summary.

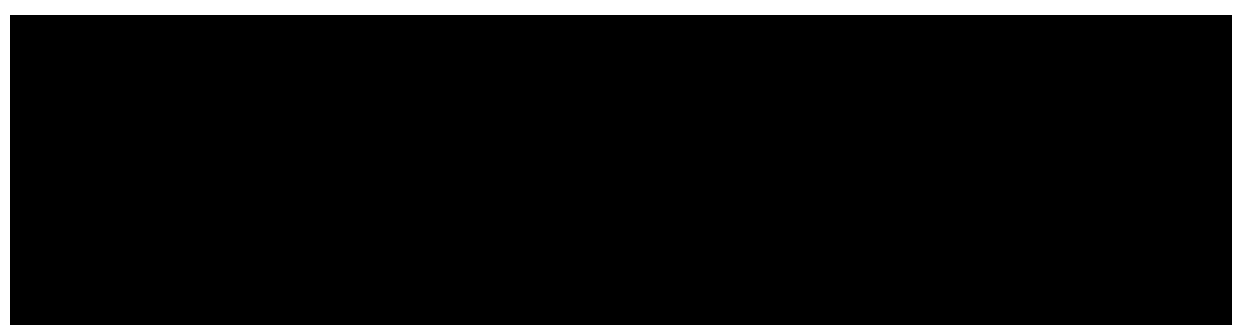

(a) DEAF

(b) HEARING

Figure 6.1: Variant HMH of DEAF and HEARING

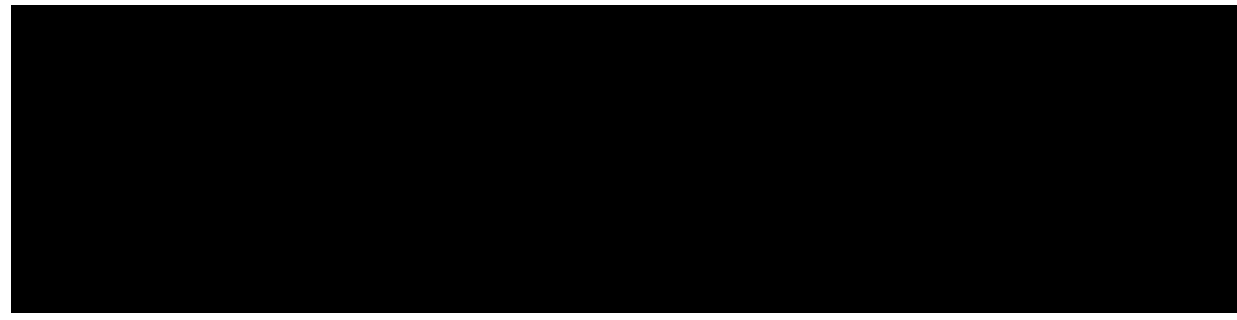

(a) DEAF

(b) HEARING

Figure 6.2: Variant MMM of DEAF and HEARING

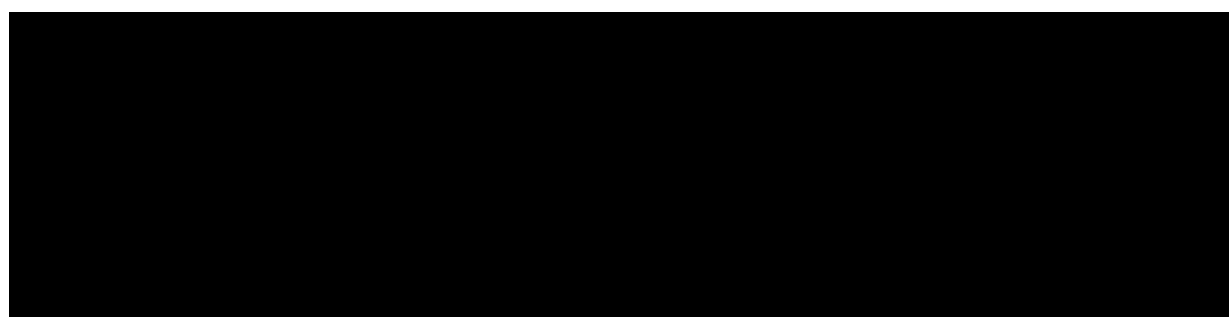

(a) DEAF

(b) HEARING

Figure 6.3: Variant MH of DEAF and HEARING 


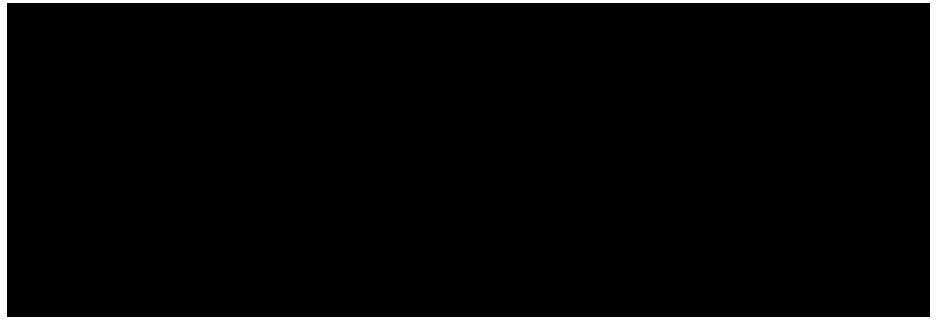

(a) DEAF

(b) HEARING

Figure 6.4: Variant M of DEAF and HEARING

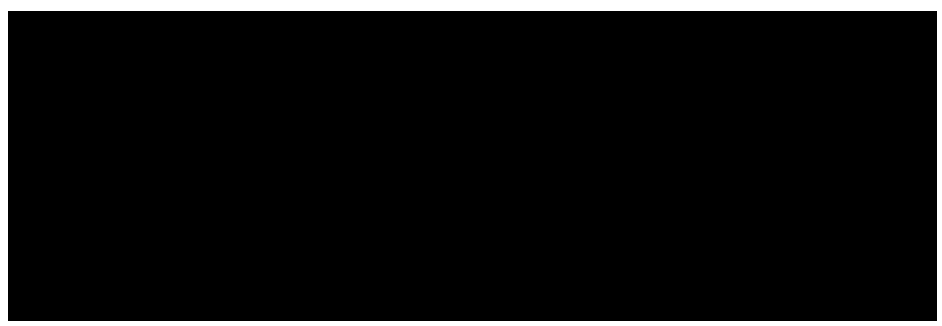

(a) DEAF

(b) HEARING

Figure 6.5: Variant H of DEAF and HEARING

\subsection{Results of lexical data}

In the preceding chapter, I have reported and discussed the findings of the analysis of the lexical elicitation task, which involved 65 informants. I correlated the lexical variants with three main social factors: school, age, and gender. Here I present the findings of the two items DEAF and HEARING which I have not discussed previously. The variants are coded in terms of their movement patterns as described in Chapter 4.

In total, there are 70 tokens of DEAF and 66 tokens of HEARING for 
analysis. ${ }^{2}$ Figures 6.6 and 6.7 illustrate the frequency distribution of the different variants across the two signs. We can see that only DEAF has five variants. The variant $\mathrm{MH}$ is produced by only one signer in DEAF and none in HEARING. Ignoring the variant $\mathrm{MH}$, we can see that both signs have the variant $\mathrm{H}$ as the least frequent variant $(9 \%$ in DEAF and 3\% in HEARING) and $M$ as the most frequent variant (39\% in DEAF and $36 \%$ in HEARING). Although there are more $\mathrm{M}$ and $\mathrm{H}$ in DEAF than in HEARING and more HMH and MMM in HEARING than in DEAF, the difference is not significant. Although the $\mathrm{MH}$ and $\mathrm{H}$ variants only occur at very low frequencies in the lexical elicitation task, we will see that they are more frequent in the conversation data, hence I want to retain them in the discussion here.

With regard to the social factors, both signs seem to show similar correlations with school, age, and gender. Since there is only one token of the variant $\mathrm{MH}$ found in DEAF and none in HEARING, it is excluded from this analysis. Figures 6.8 and 6.9 demonstrate the distribution of variants by school in DEAF and HEARING respectively. It is apparent in both items that HSD uses more of the variant $M$ than other schools and CMS uses more of the variant $\mathrm{HMH}$. Moreover, OMS seems to use a bit more MMM in HEARING than in DEAF.

\footnotetext{
${ }^{2}$ The collected tokens for DEAF and HEARING are 72 and 68 respectively. As with the lexical analysis in Chapter 5, VSD was not included to avoid double-counting and one VSD participant was excluded (see Section 5.4.1.1 for explanation). One token of DEAF that resembles the ASL form rather than HKSL was also excluded.
} 


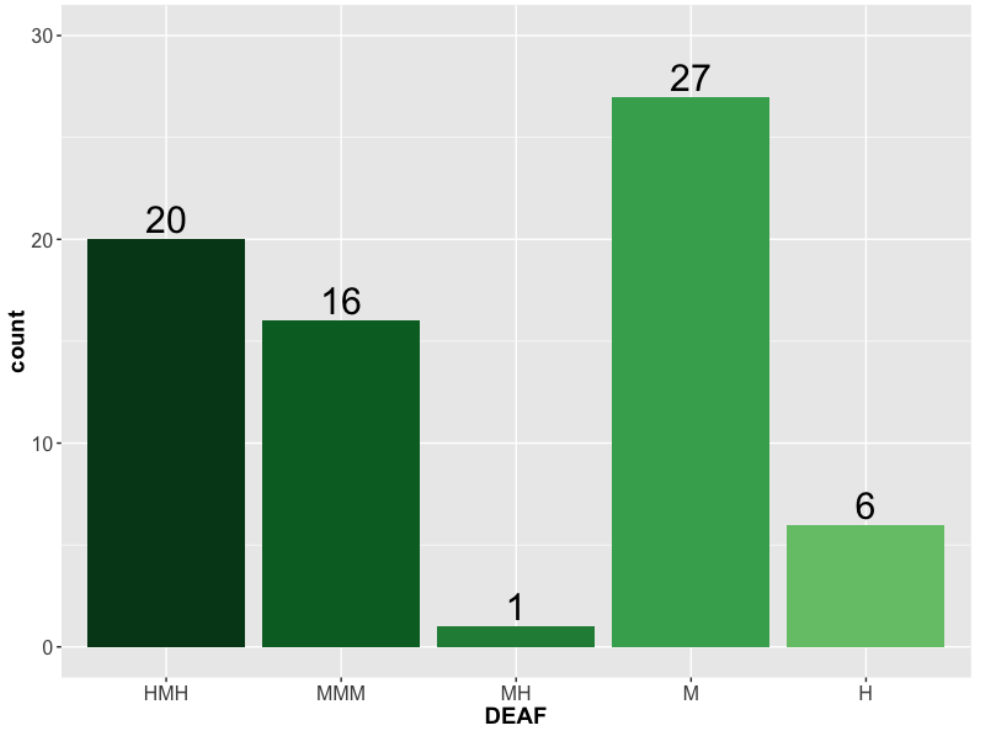

Figure 6.6: Frequency distribution of variants of DEAF (lexical data)

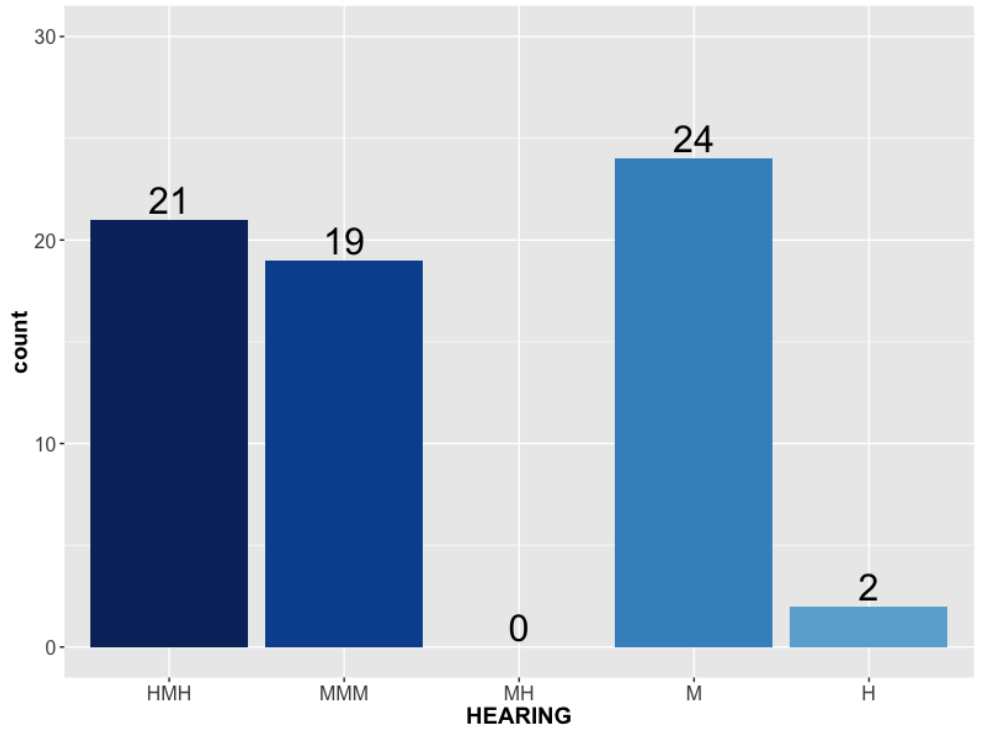

Figure 6.7: Frequency distribution of variants of HEARING (lexical data) 


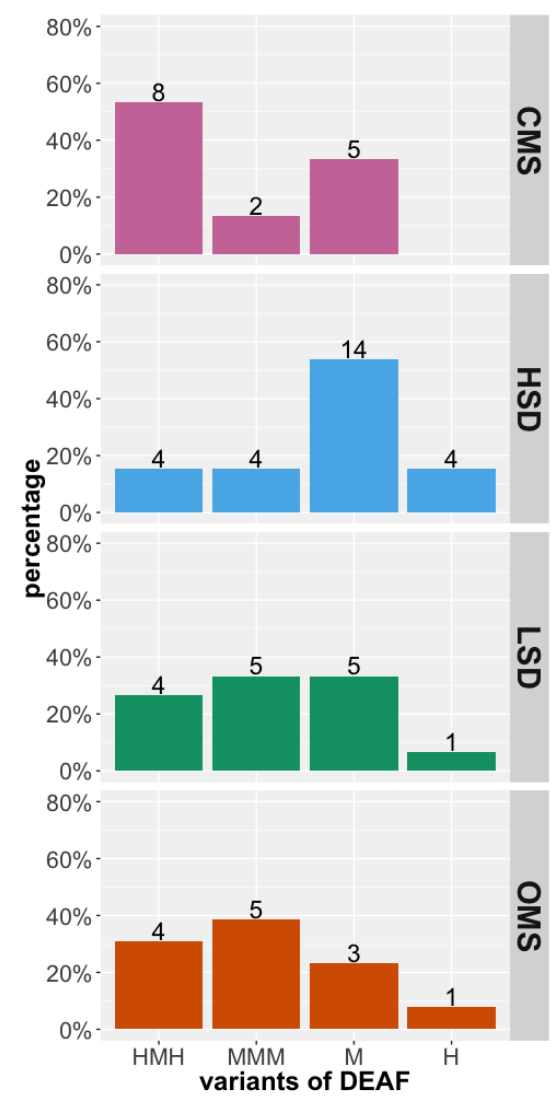

Figure 6.8: Distribution of variants of DEAF by school (lexical data)

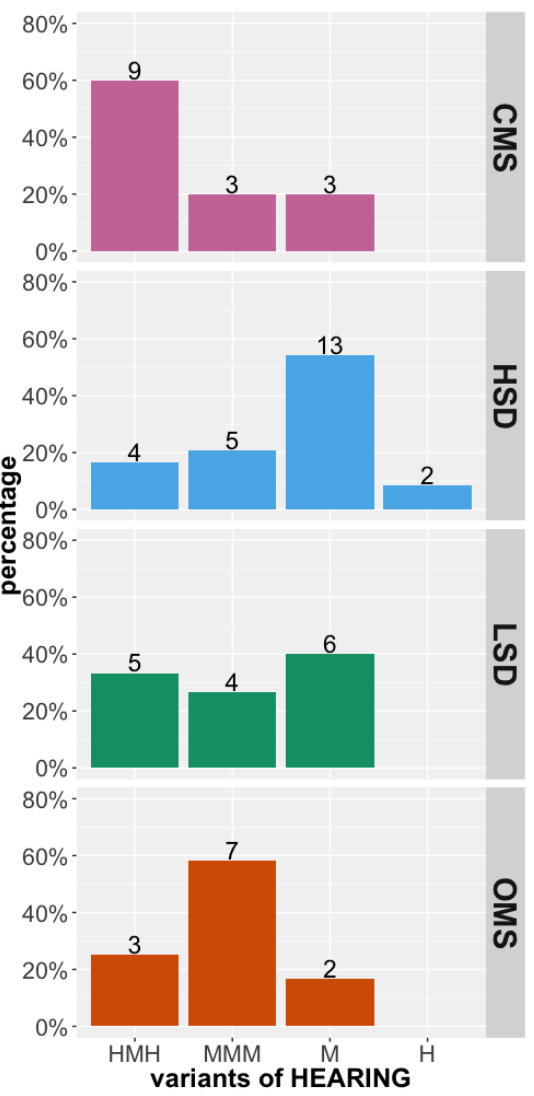

Figure 6.9: Distribution of variants of HEARING by school (lexical data)

The distribution of DEAF and HEARING variants by age are displayed in Figures 6.10 and 6.11. The younger signers prefer the variant $M$ in both DEAF and HEARING. Interestingly, the traditional form $\mathrm{HMH}$ is favoured by the middle-aged signers, but not the older ones. The older signers slightly prefer MMM. 


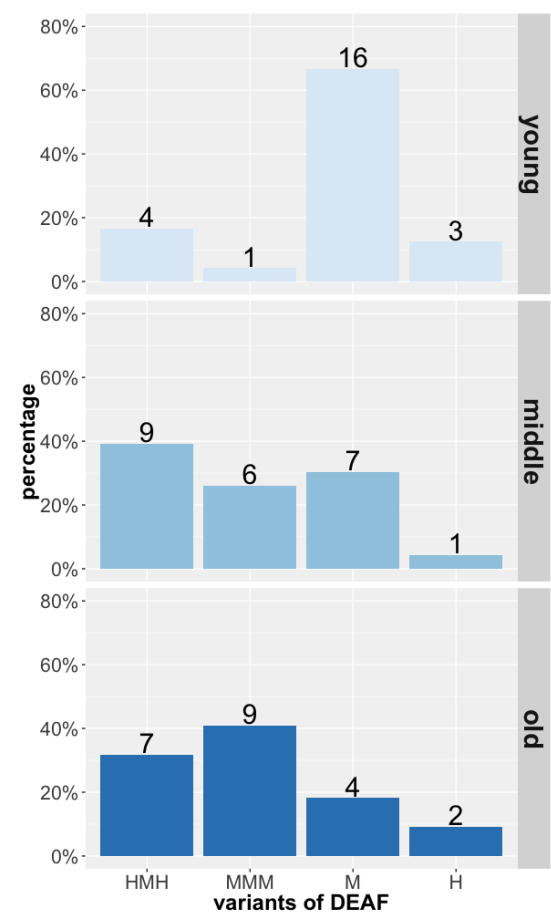

Figure 6.10: Distribution of variants of DEAF by age (lexical data)

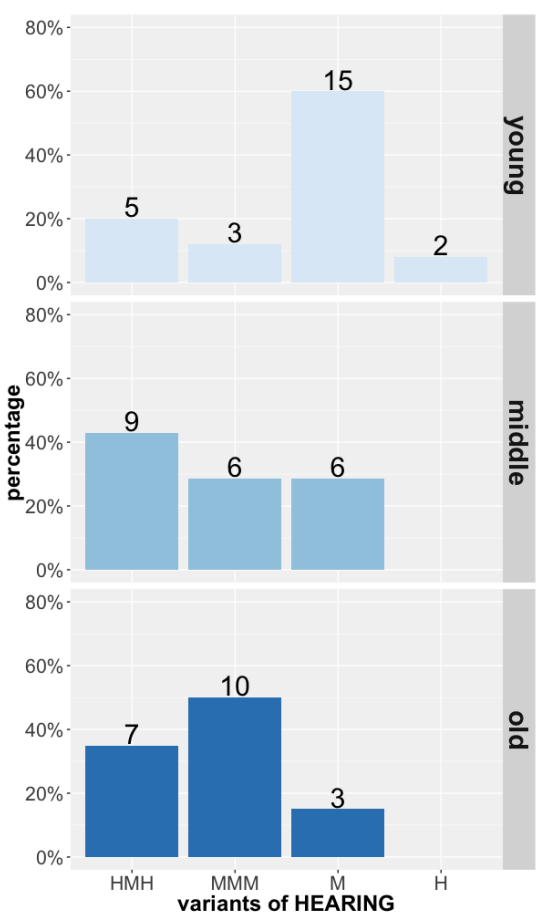

Figure 6.11: Distribution of variants of HEARING by age (lexical data)

Lastly, Figures 6.12 and 6.13 show the distribution of variants by gender. In both DEAF and HEARING, women tend to use more of the variant M, while men favour HMH a bit more. Summarizing the results so far, it appears that there is a shift from the traditional form $\mathrm{HMH}$ to the innovative form $\mathrm{M}$ in both items, and as we can see in Table 6.1, the young female signers from HSD may be the leaders of such change. I now turn to the results of the two phonological variables analyzed from the conversation data.

\begin{tabular}{lccccc}
\hline & HMH & MMM & M & H & total \\
\hline Female & 1 & 2 & 9 & 1 & 13 \\
Male & 3 & 2 & 5 & 3 & 13 \\
\hline
\end{tabular}

Table 6.1: Distribution of variants of DEAF by gender for HSD signers (lexical data) 


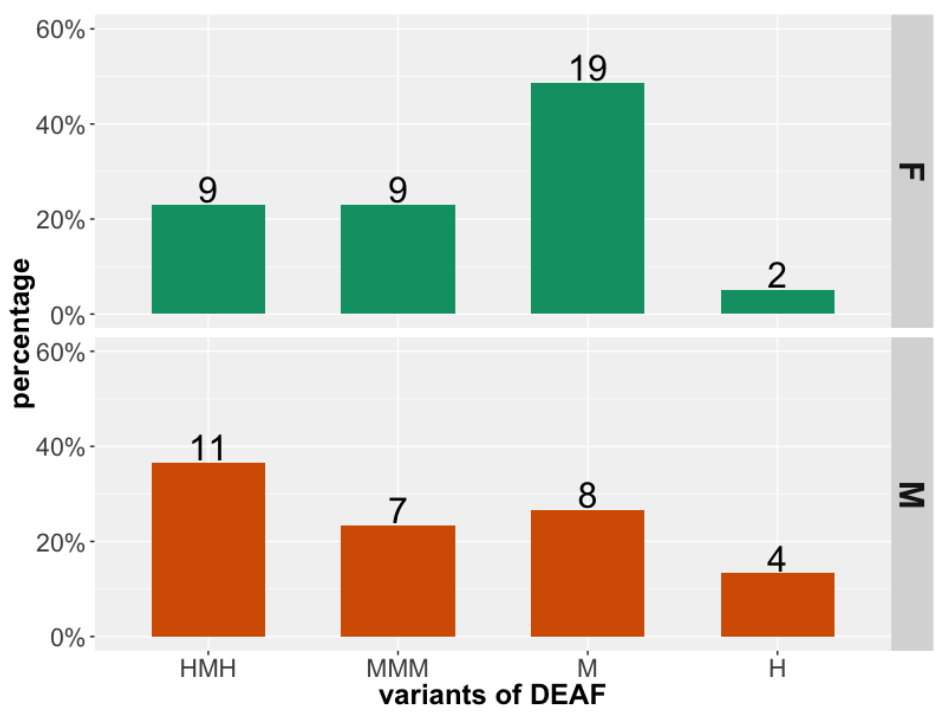

Figure 6.12: Distribution of variants of DEAF by gender (lexical data)

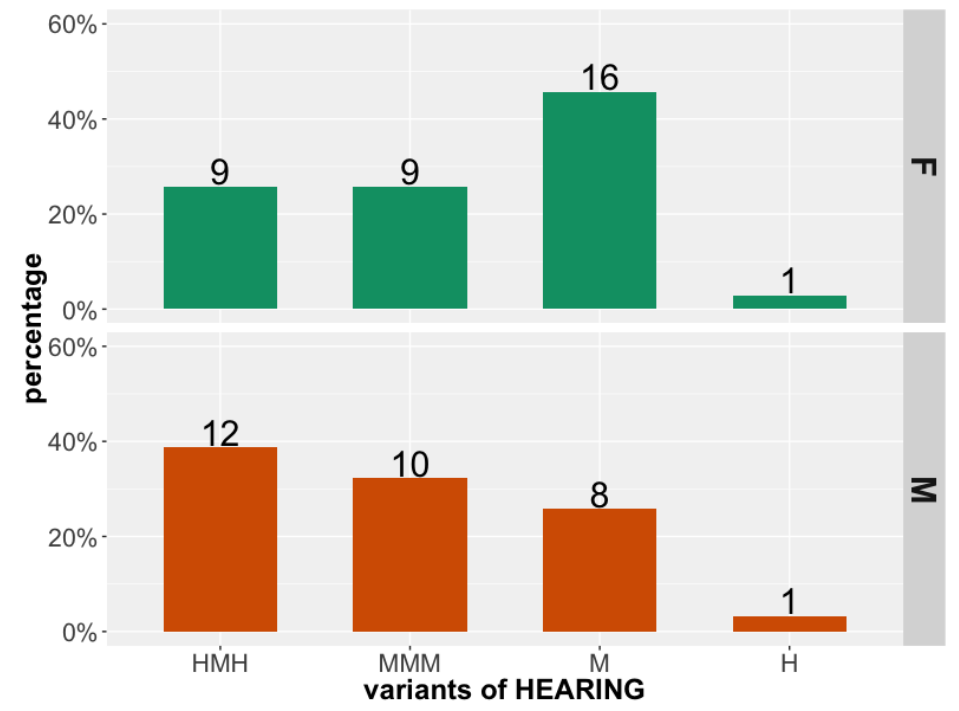

Figure 6.13: Distribution of variants of HEARING by gender (lexical data) 


\subsection{Overall results of conversation data}

Overall, there are a total of 580 tokens of DEAF and 365 tokens of HEARING collected from 40 signers. The number of tokens given by individual participants varies from 2 to 52 for DEAF and from 1 to 35 for HEARING. The distribution across individuals is illustrated in Figures 6.14 and 6.15. The large variance between individual signers is due to the nature of the recorded conversation. The participants were asked to talk about anything they like during the free conversation period. While some of them had a casual conversation about cooking, playing games, and so on, some were consciously talking about deaf related topics, such as their experience in deaf school. Hence, some participants did not produce any tokens in the conversation and I had to code the interview data for more tokens (as explained in Section 4.5.2.1). ${ }^{3}$ It is important to keep this variance in mind when interpreting the results reported in the coming sections.

\footnotetext{
${ }^{3}$ Hence, in this chapter, the conversation data refers to the conversation data and some interview data coded for this variable.
} 


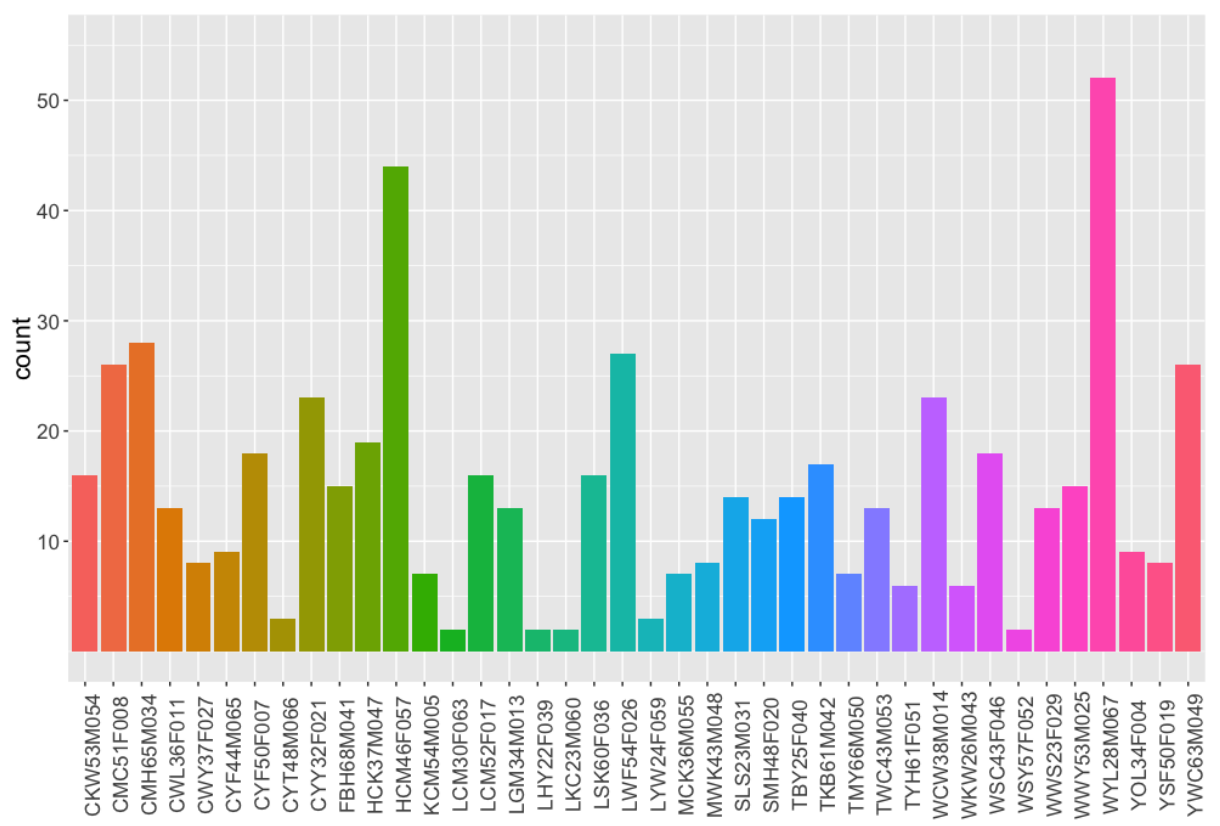

Figure 6.14: Frequency distribution of DEAF by individual participants (conversation data)

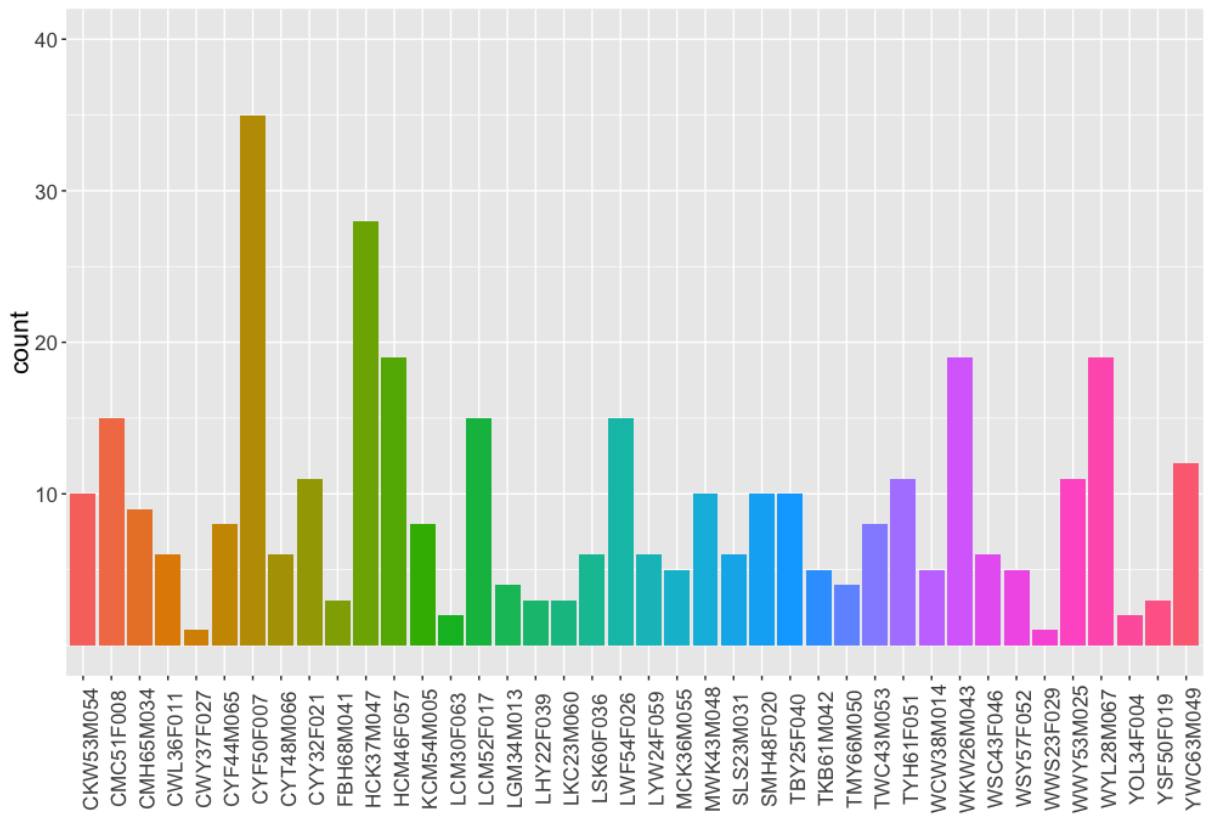

Figure 6.15: Frequency distribution of HEARING by individual participants (conversation data) 
Both signs have five different variants in terms of movement pattern and they are coded as $\mathrm{HMH}, \mathrm{MMM}, \mathrm{MH}, \mathrm{M}$, and $\mathrm{H}$ (see Section 4.5.2.1 for the description of each variant). Figures 6.16 and 6.17 demonstrate the distribution of the five variants of DEAF and HEARING respectively. We can see that the distribution patterns of the two signs are quite different. In DEAF, the most popular variant is $\mathrm{H}$; while in HEARING, $\mathrm{MH}$ is the most frequent one. Both variants ( $\mathrm{H}$ in DEAF and $\mathrm{MH}$ in HEARING) constitute around $32 \%$ of the total. On the other hand, MMM is the least frequent variant in DEAF, just $4 \%$ of the total tokens. In HEARING, both $\mathrm{H}$ and $\mathrm{M}$ have the lowest percentage (both 15\%).

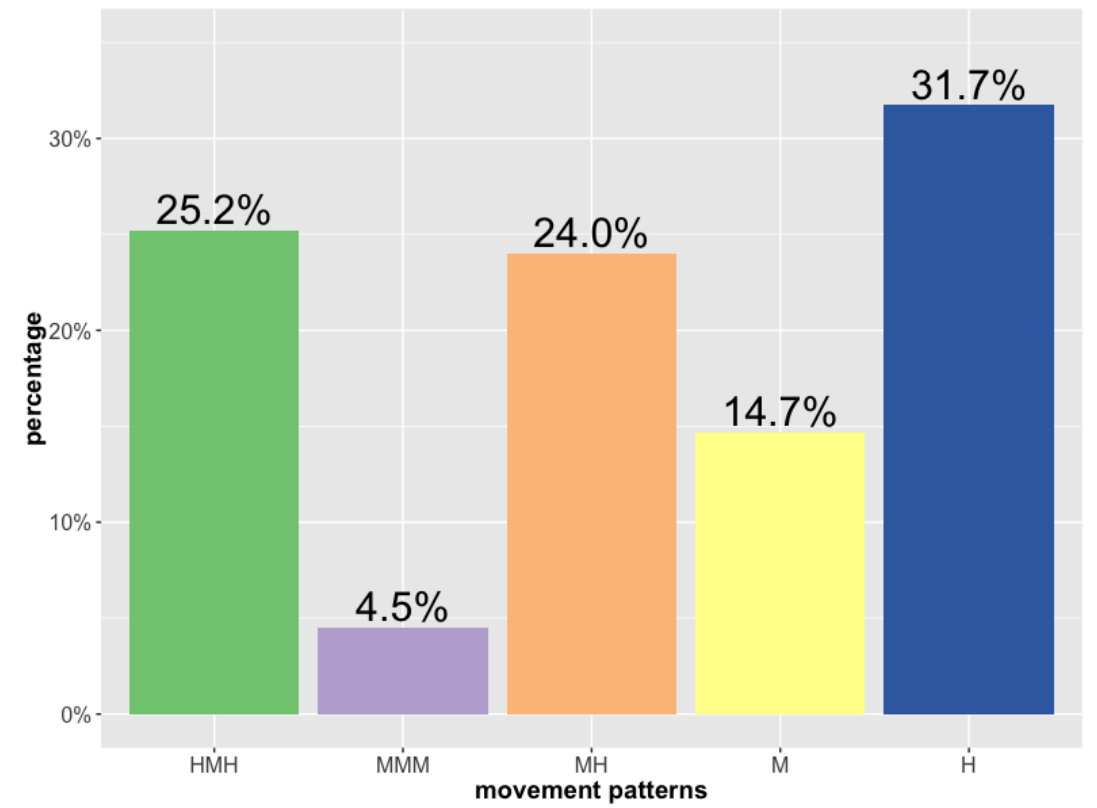

Figure 6.16: Percentage distribution of variants of DEAF (conversation data) 


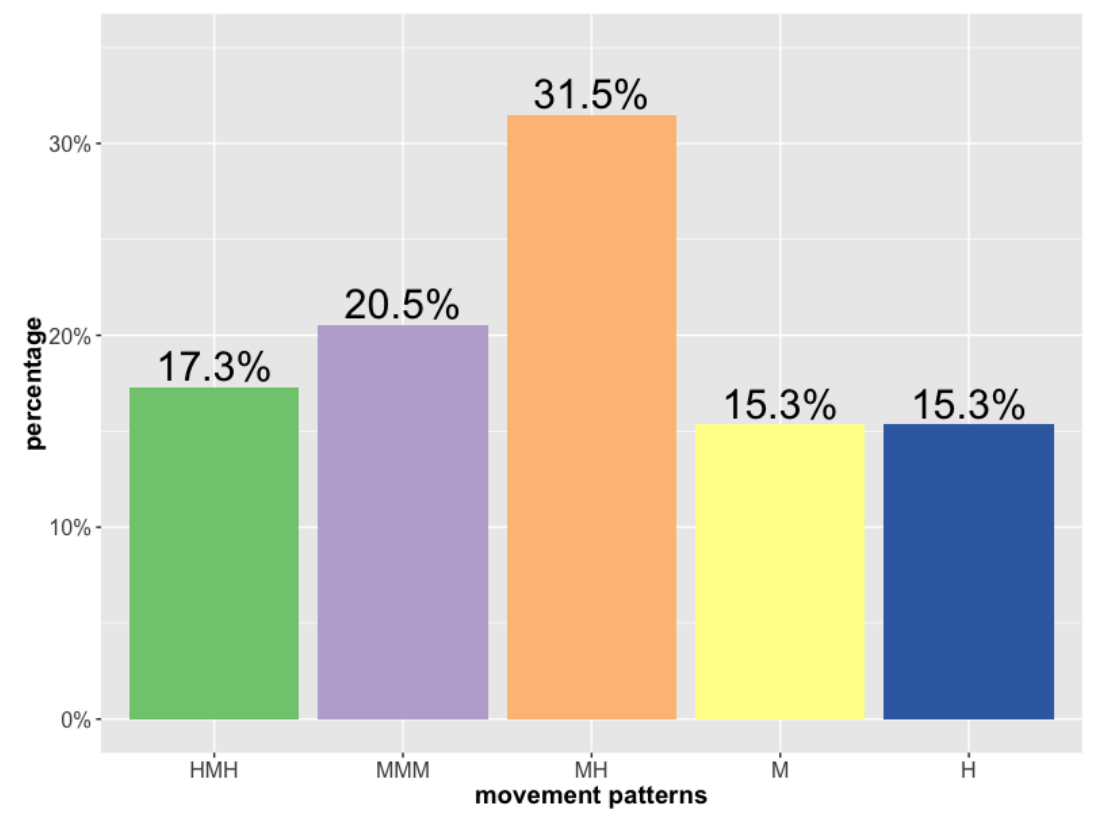

Figure 6.17: Percentage distribution of variants of HEARING (conversation data)

The variants that are most common in the conversation data are exactly the ones that were produced least often in direct lexical elicitation (Figures 6.6 and 6.7 on page 223). The variants elicited are supposed to be the most salient ones in the community, i.e., they are the forms signers are most consciously aware of. Given this definition of salience, the least salient forms are used most in the conversation, $\mathrm{H}$ in DEAF and MH in in HEARING (Figures 6.16 and 6.17). One possibility is that $\mathrm{H}$ (the most reduced variant) and $\mathrm{MH}$ (the second most reduced variant) are particularly favoured in very specific and often derived contexts, e.g., compounds (we will see in Section 6.4.1 that this has been argued for ASL). Another possibility is that $\mathrm{H}$ and $\mathrm{MH}$ are especially favoured in contexts in conversation where the immediate phonological environment is influencing the production of a reduced (or somewhat reduced) variant.

When we look at the distribution of the five variants across individual 
signers in Figures 6.18 and 6.19, we can see that MMM in DEAF is mainly produced by several signers, but a lot more participants produced MMM in HEARING. Table 6.2 outlines the number of participants that produced the corresponding variants of DEAF and HEARING. Except for MMM in DEAF, which is produced by seven signers (17.5\%), all other variants (both DEAF and HEARING) are produced by at least $45 \%$ of the participants, with the highest by $82.5 \%$. In addition, while three variants of DEAF were produced by at least $75 \%$ of the signers $(\mathrm{H}, \mathrm{HMH}$, and $\mathrm{MH})$, only one variant of HEARING $(\mathrm{MH})$ was made by $80 \%$ of the signers. The other four variants were produced by $45 \%$ to $60 \%$ of the participants.

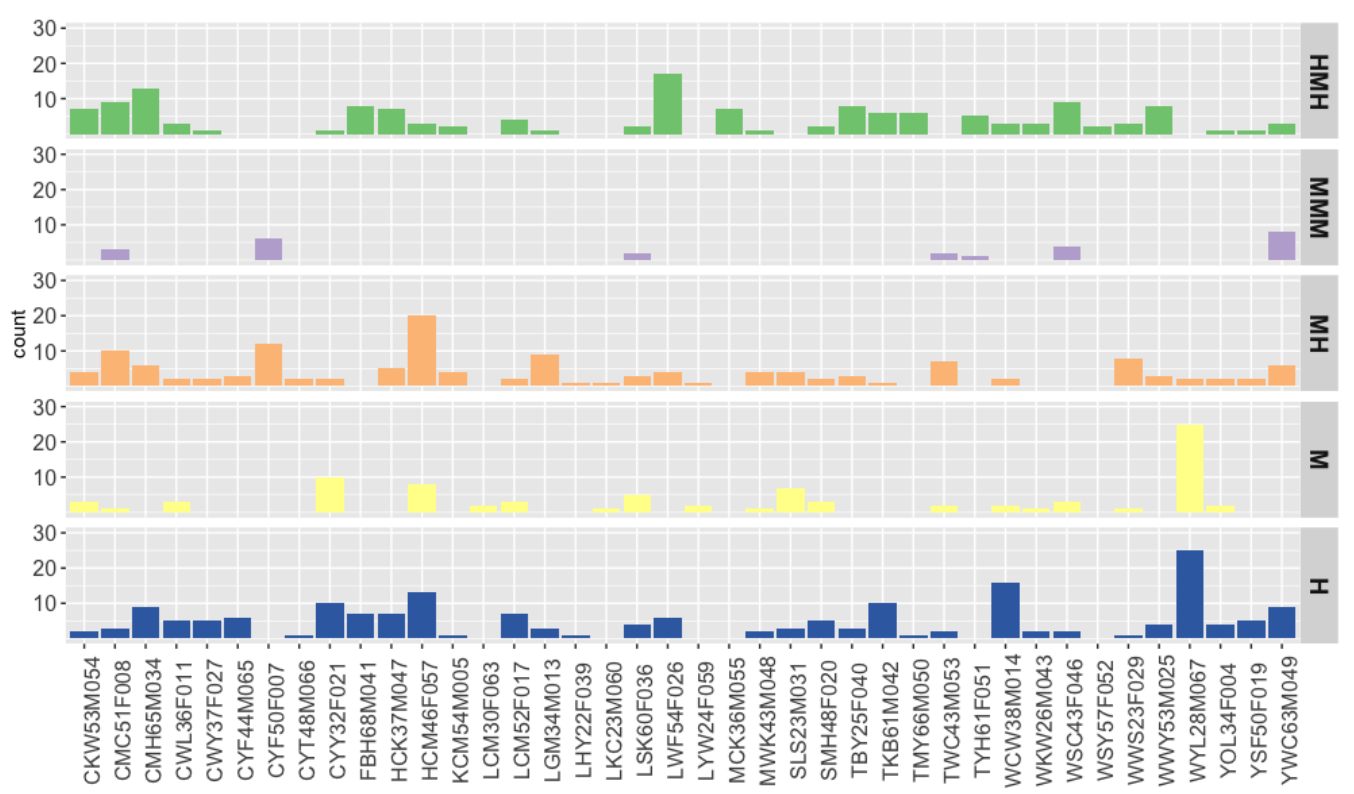

Figure 6.18: Distribution of variants of DEAF by individual participants (conversation data) 


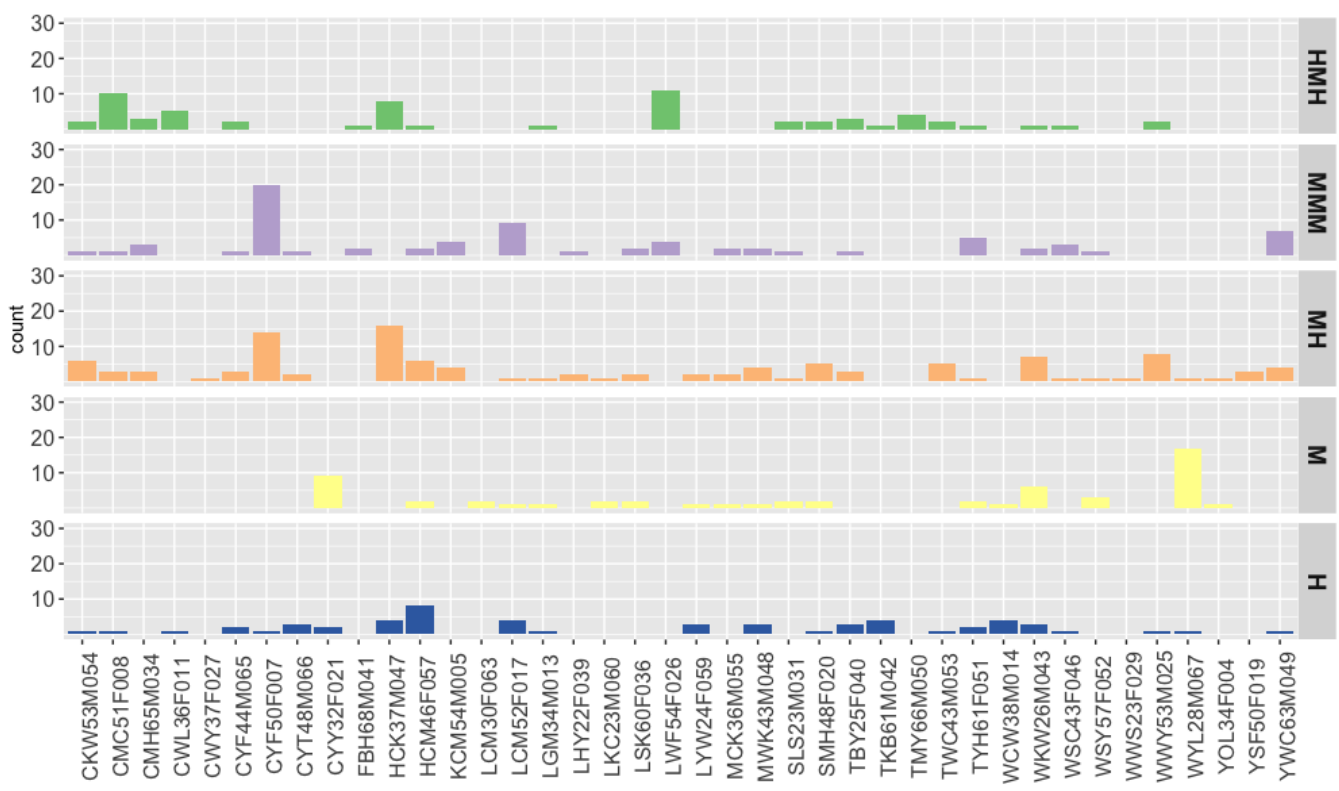

Figure 6.19: Distribution of variants of HEARING by individual participants (conversation data)

\begin{tabular}{lcc}
\hline Variants & DEAF & HEARING \\
\hline H & $33(82.5 \%)$ & $24(60 \%)$ \\
HMH & $30(75.0 \%)$ & $20(50 \%)$ \\
M & $20(50.0 \%)$ & $18(45 \%)$ \\
MH & $32(80.0 \%)$ & $32(80 \%)$ \\
MMM & $7(17.5 \%)$ & $22(55 \%)$ \\
\hline
\end{tabular}

Table 6.2: Numbers of participants producing the five variants (conversation data)

In sum, the two signs DEAF and HEARING have pretty much the same variants but the variants $\mathrm{H}, \mathrm{HMH}$, and $\mathrm{MMM}$ are distributed quite differently in the conversation data, even though the findings of their lexical analysis (reported in Section 6.2) look quite similar. 


\subsubsection{Linguistic factors}

As stated in Chapter 4, due to the small size and imbalanced nature of the data, the relationship between the variables and the independent variables (i.e., both linguistic and social factors) needs to be explored by using crosstabulation. A Fisher Exact test or Chi-square test is then adopted to test the statistical significance of the potential association.

In DEAF, three linguistic factors demonstrate a significant relation with the five variants in an initial analysis of the contingency tables. These factors are grammatical class, change in hand dominance, and genre. However, when we examine the data closely, some associations may not be plausible. For the factor change in hand dominance (Table 6.3), 89\% of the tokens have no change in hand dominance (517 out of 580) and those which involved change both before and after the target sign comprised less than $1 \%$ of the tokens (5 out of 580). Also, over half of the cells have five or less tokens (three of them have zero tokens). This will probably affect the accuracy of any test. Yet, the contingency table reveals that the variant $\mathrm{MH}$ occurs more when there is a change in hand dominance, no matter whether this is after, before, or both, as compared with other variants.

\begin{tabular}{lrrrrrr}
\hline $\mathrm{p}<0.05$ & $\mathrm{H}$ & \multicolumn{1}{c}{$\mathrm{HMH}$} & $\mathrm{M}$ & $\mathrm{MH}$ & $\mathrm{MMM}$ & total \\
\hline after & $4(18 \%)$ & $3(14 \%)$ & $1(5 \%)$ & $12(54 \%)$ & $2(9 \%)$ & 22 \\
before & $12(33 \%)$ & $6(17 \%)$ & $5(14 \%)$ & $13(36 \%)$ & & 36 \\
both & & $1(20 \%)$ & $1(20 \%)$ & $3(60 \%)$ & & 5 \\
no & $168(33 \%)$ & $136(26 \%)$ & $78(15 \%)$ & $111(21 \%)$ & $24(5 \%)$ & 517 \\
\hline total & 184 & 146 & 85 & 139 & 26 & 580 \\
\hline
\end{tabular}

Table 6.3: Contingency table for change in hand dominance and variants of DEAF (conversation data) 
For the factor of genre, although there is no problem with cell values, the test results may not be trustworthy because of the varying numbers of tokens provided by individuals (as demonstrated in Section 6.3). Indeed, the reason for including interview data is due to the very low number of tokens obtained from certain signers in the conversation data. Hence, any difference observed is very likely connected to individuals rather than the genre. This applies to the sign HEARING as well. I now turn to HEARING and will discuss the factor grammatical class together with the findings of HEARING.

In HEARING, the preliminary results show that four linguistic factors are significantly associated with the five variants. They are grammatical class, following segment, location of the following segment, and genre. The issue of genre has just been explained in the preceding paragraph. For the factor of following segment, the majority of the tokens are signs (97.5\%). As shown in Table 6.4, the variant MMM occurs more frequently with following pause. Again, the result should be interpreted with caution due to the small number of pauses.

\begin{tabular}{lcccrrr}
\hline $\mathrm{p}<0.05$ & $\mathrm{H}$ & $\mathrm{HMH}$ & $\mathrm{M}$ & $\mathrm{MH}$ & $\mathrm{MMM}$ & total \\
\hline pause & & $1(11 \%)$ & $1(11 \%)$ & $1(11 \%)$ & $6(67 \%)$ & 9 \\
sign & $56(16 \%)$ & $62(17 \%)$ & $55(15 \%)$ & $114(32 \%)$ & $69(19 \%)$ & 356 \\
\hline total & 56 & 63 & 56 & 115 & \multicolumn{1}{c}{75} & 365 \\
\hline
\end{tabular}

Table 6.4: Contingency table for following segment and variants of HEARING (conversation data)

The other linguistic factor also relates to the following phonological environment. Table 6.5 tabulates the five variants by the following location excluding pauses. At first glance, the form $\mathrm{MH}$ occurs more frequently 
if the following location is middle (between ear and chin) and low (chin or below). However, after checking the location of the target signs, this correlation may be linked to the location rather than the movement pattern. Among the 65 tokens of the $\mathrm{MH}$ variant which occur before a sign located at a low location, $80 \%$ are finished at a low location too; for those followed by a sign located at a middle location, 30 out of the 48 tokens $(62.5 \%)$ are also ended at a middle location. It is therefore possible that location assimilation has occurred. Hence, this may be an association between the location of the target signs and the location of the following segment, rather than an association between the movement pattern of the variant and the following location.

\begin{tabular}{lrrrrrr}
\hline $\mathrm{p}<0.05$ & $\mathrm{H}$ & $\mathrm{HMH}$ & $\mathrm{M}$ & \multicolumn{1}{c}{$\mathrm{MH}$} & $\mathrm{MMM}$ & total \\
\hline high & $3(25 \%)$ & $3(25 \%)$ & $1(8 \%)$ & $1(8 \%)$ & $4(33 \%)$ & 12 \\
middle & $14(12 \%)$ & $27(22 \%)$ & $18(15 \%)$ & $48(39 \%)$ & $15(12 \%)$ & 122 \\
low & $39(18 \%)$ & $32(14 \%)$ & $36(16 \%)$ & $65(29 \%)$ & $50(23 \%)$ & 222 \\
\hline total & 56 & 62 & 55 & 114 & \multicolumn{1}{l}{69} & 356 \\
\hline
\end{tabular}

Table 6.5: Contingency table for following location and variants of HEARING (conversation data)

Finally, returning to grammatical class, this is found to be a significant factor in both DEAF and HEARING. The contingency tables for grammatical class and the two signs are displayed in Tables 6.6 and 6.7. We can see that the distributions of the two variables demonstrate both similarities and dissimilarities. Noun constitutes over $80 \%$ of the data in both DEAF and HEARING, and compound is the least common, 4\% in DEAF and $3 \%$ in HEARING. For both lexemes, the form MMM never occurs in compounds. This is quite reasonable as MMM is the form with the most movement, whereas in compound signs, movement tends to be reduced. 
On the other hand, while the variant $\mathrm{H}$ occurs more frequently in DEAF compounds, HMH is more frequent in HEARING compounds. Furthermore, both $\mathrm{HMH}(34 \%)$ and $\mathrm{M}(31 \%)$ appear more frequently in predicate adjectives of DEAF, but HMH never appears in that of HEARING, instead the forms MMM (50\%) and M (45\%) are more frequent in its predicate adjectives. Nonetheless, the different patterns shown in these two cross tabulations seem to be in accordance with the overall distribution reported previously.

\begin{tabular}{|c|c|c|c|c|c|c|}
\hline $\mathrm{p}<0.01$ & $\mathrm{H}$ & $\mathrm{HMH}$ & M & MH & MMM & total \\
\hline noun & $153(33 \%)$ & $115(25 \%)$ & $58(13 \%)$ & $122(26 \%)$ & $17(4 \%)$ & 465 \\
\hline adjective & $14(24 \%)$ & $15(26 \%)$ & $11(19 \%)$ & $12(21 \%)$ & $6(10 \%)$ & 58 \\
\hline predicate adjective & $6(19 \%)$ & $11(34 \%)$ & $10(31 \%)$ & $2(6 \%)$ & $3(9 \%)$ & 32 \\
\hline compound & $11(44 \%)$ & $5(20 \%)$ & $6(24 \%)$ & $3(12 \%)$ & & 25 \\
\hline total & 184 & 146 & 85 & 139 & 26 & 580 \\
\hline
\end{tabular}

Table 6.6: Contingency table for grammatical class and variants of DEAF (conversation data)

\begin{tabular}{lrrrrrr}
\hline $\mathrm{p}<0.001$ & $\mathrm{H}$ & \multicolumn{1}{c}{$\mathrm{HMH}$} & $\mathrm{M}$ & $\mathrm{MH}$ & $\mathrm{MMM}$ & total \\
\hline noun & $51(17 \%)$ & $51(17 \%)$ & $40(13 \%)$ & $105(34 \%)$ & $57(19 \%)$ & 304 \\
adjective & $2(7 \%)$ & $7(24 \%)$ & $6(21 \%)$ & $6(21 \%)$ & $8(27 \%)$ & 29 \\
predicate adjective & & & $9(45 \%)$ & $1(5 \%)$ & $10(50 \%)$ & 20 \\
compound & $3(25 \%)$ & $5(42 \%)$ & $1(8 \%)$ & $3(25 \%)$ & & 12 \\
\hline total & 56 & 63 & \multicolumn{1}{c}{56} & \multicolumn{1}{c}{115} & \multicolumn{1}{c}{75} & 365 \\
\hline
\end{tabular}

Table 6.7: Contingency table for grammatical class and variants of HEARING (conversation data)

\subsubsection{Social factors}

Regarding the social factors, the distributional analysis also shows both differences and parallels between the two variables. Figures 6.20 and 6.21 
illustrate the variants' distribution by school for DEAF and HEARING respectively (both $\mathrm{p}<0.00001)$. We can see that in DEAF, both CMS $(38 \%)$ and HSD (34\%) favour the variant $\mathrm{H}$ most, OMS (40\%) prefers $\mathrm{HMH}$, and LSD likes both $\mathrm{H}(31 \%)$ and $\mathrm{MH}(31 \%)$ a little more than the other forms. However, their preference shifts in HEARING, CMS (30\%) and LSD (53\%) prefer $\mathrm{MH}$, HSD favours M (35\%) and OMS favours MMM (32\%). In both DEAF and HEARING, the variants HMH and MMM are favoured by OMS signers (49\% of HMH and 54\% of MMM in DEAF; $49 \%$ of $\mathrm{HMH}$ and $48 \%$ of MMM in HEARING), whereas the form $\mathrm{M}$ is favoured by HSD (54\% in DEAF and $41 \%$ in HEARING).

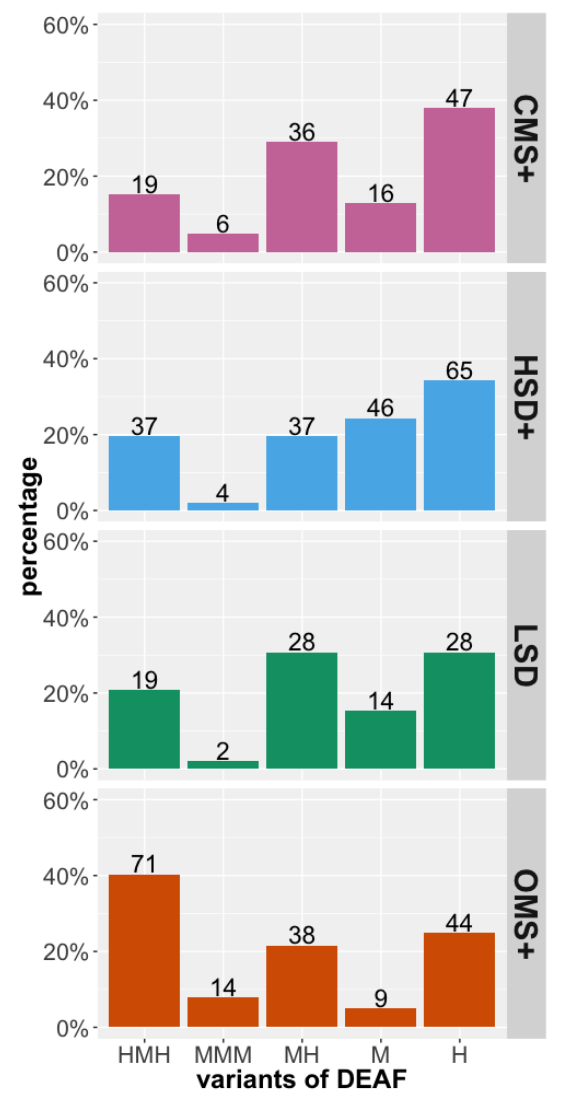

Figure 6.20: Distribution of variants of DEAF by school (conversation data) (Fisher's Exact test, $\mathrm{p}<0.00001$ )

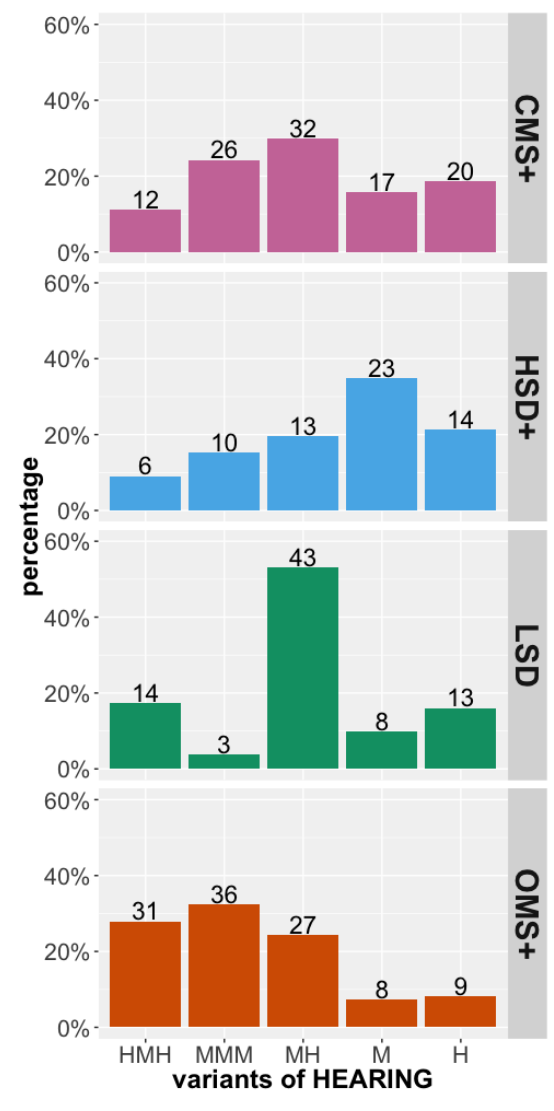

Figure 6.21: Distribution of variants of HEARING by school (conversation data) (Fisher's Exact test, $\mathrm{p}<0.00001$ ) 
Similarly, different age groups have different preferences with respect to DEAF and HEARING and both variables demonstrate statistical significance with p-values much smaller than 0.05 (both $\mathrm{p}<0.0001$ ). Figures 6.22 and 6.23 illustrate their differentiation. In DEAF, both younger and middleaged groups use the variant $H$ the most (34\% and $36 \%$ respectively) and the older group prefers HMH (37\%). On the other hand, in HEARING, the variants $\mathrm{M}, \mathrm{MH}$, and $\mathrm{MMM}$ are favoured by the younger, middle-aged, and older groups respectively $(48 \%, 40 \%$, and $35 \%$ correspondingly). It should be noted that the form MMM is never produced by the younger signers in DEAF. In addition, the older signers used a lot more $\mathrm{HMH}$ than MMM (37\% versus 8\%) in DEAF, but more MMM than HMH (35\% versus $21 \%$ ) in HEARING. Likewise, when we compare the three variants $\mathrm{HMH}$, MMM, and $\mathrm{M}$, the first two forms (HMH and MMM) are favoured by the older signers (64\% of $\mathrm{HMH}$ and $77 \%$ of MMM in DEAF; $56 \%$ of $\mathrm{HMH}$ and $79 \%$ of MMM in HEARING) and $\mathrm{M}$ is favoured by the younger ones in both DEAF (60\%) and HEARING (73\%).

Unlike school and age, gender is found to be a significant factor only in HEARING $(p<0.05)$, and not in DEAF $(p=0.0590) .{ }^{4}$ In Figure 6.24, we can see that the largest difference between men and women is observed in the variants MMM and $\mathrm{MH}$. Women use much more MMM than men, and men produced considerably more $\mathrm{MH}$ than women.

\footnotetext{
${ }^{4}$ It should be noted that the $p$-value is quite close to 0.05 (a conventional value), which may indicate a strong trend though not statistically significant. For the problems related to $\mathrm{p}$-values, please see Wagenmakers (2007).
} 


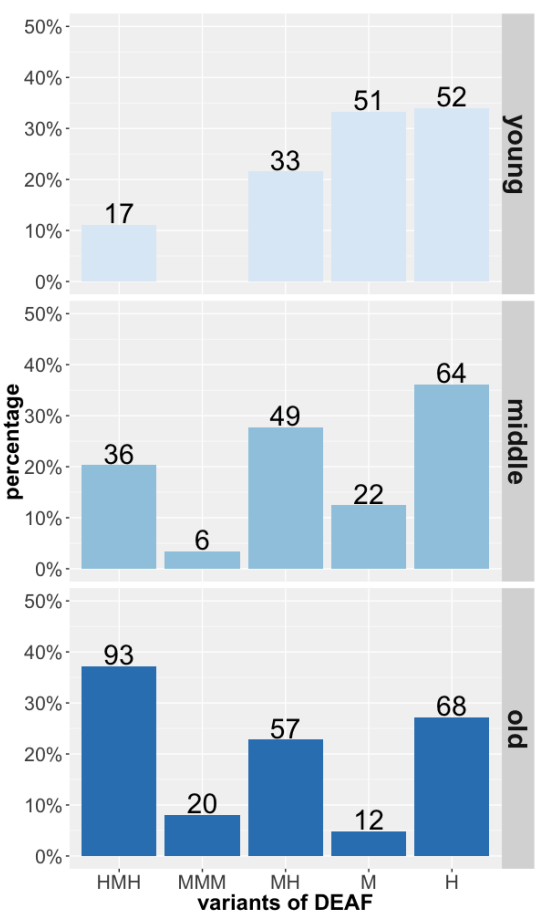

Figure 6.22: Distribution of variants of DEAF by age (conversation data) (Fisher's Exact test, $\mathrm{p}<0.0001$ )

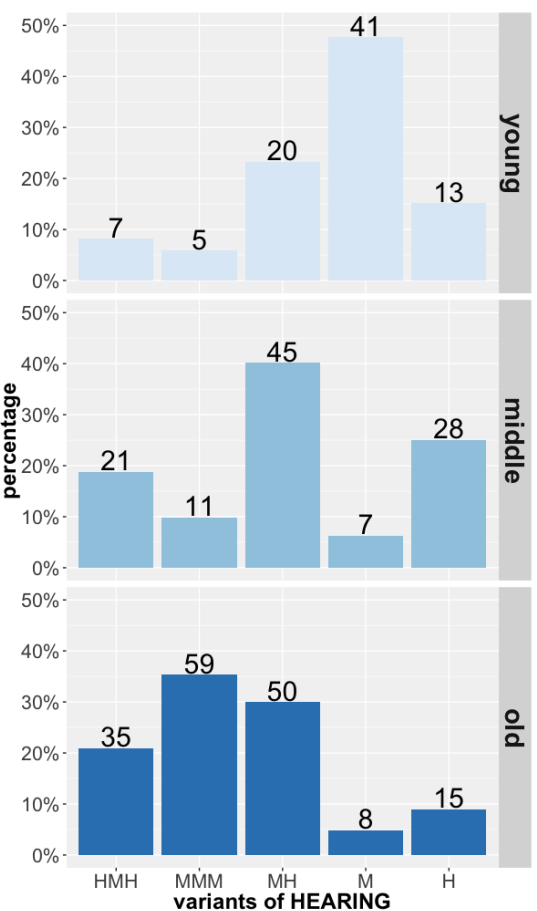

Figure 6.23: Distribution of variants of HEARING by age (conversation data) (Fisher's Exact test, $\mathrm{p}<0.0001)$

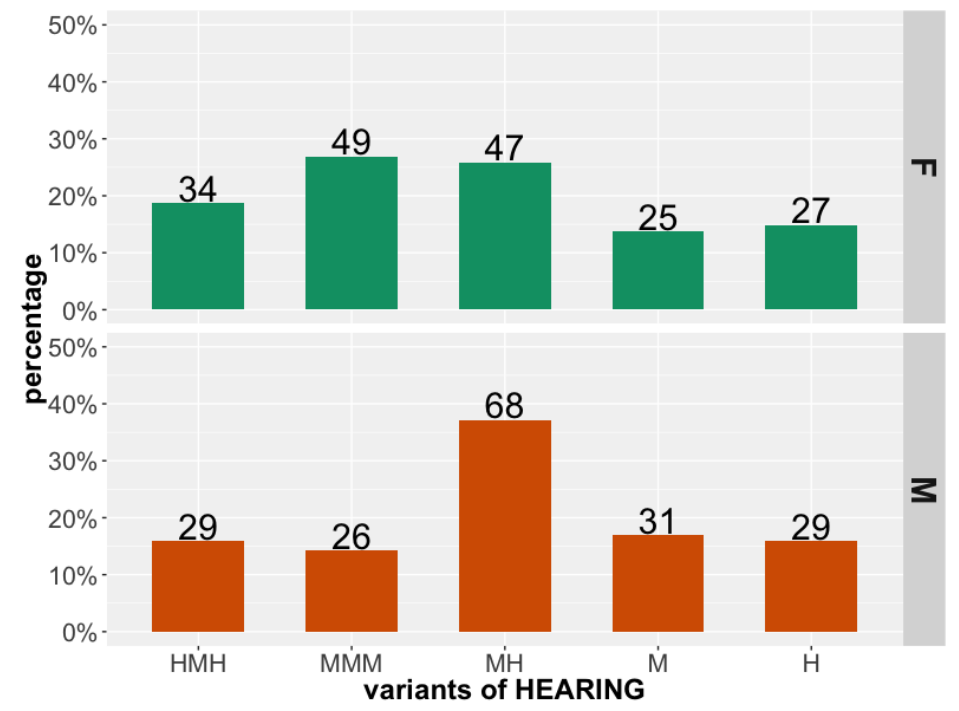

Figure 6.24: Distribution of variants of HEARING by gender (conversation data) (Fisher's Exact test, $\mathrm{p}<0.05$ ) 


\subsection{Discussion}

The results of the contingency table analysis demonstrate that the variables DEAF and HEARING in HKSL, like DEAF in ASL, are systematically conditioned by both linguistic and social factors. Nevertheless, the two variables appear to have a different distribution pattern across various factor groups. In this section, I will first compare the results of the current study with the ASL study. I will then discuss whether the variation observed in DEAF and HEARING represents a change in progress. The potential implications of the analysis of such variation will also be considered.

\subsubsection{Comparison with the ASL results}

As reviewed in Chapter 2, results of the multivariate analysis of 1,618 tokens in the ASL study showed that variation in the form of DEAF is constrained by both linguistic and social factors in a systematic way. In the first stage of their analysis, Lucas et al. (2001) found that the variant choice between citation and non-citation forms was conditioned by grammatical class and discourse genre. In the second stage, the choice between the two non-citation forms (chin-to-ear and contact-cheek) was governed by grammatical class and the location of the following sign. They found that the ear-to-chin citation form was favoured in predicate adjectives and the contact-cheek variant was favoured in compounds. They reasoned that predicate adjectives are normally in an emphatic position, so discourse constraints may play a role in favouring the citation forms, whereas the reduced contact-cheek form was very likely associated with compounding 
process. On the other hand, when the location of the following sign was between the ear and chin, the contact-cheek variant was disfavoured. They used the Obligatory Contour Principle ${ }^{5}$ to explain this result, reasoning that a distinction between DEAF and the following sign was needed (Lucas et al., 2001, p. 121).

Similar results are observed in the current data with respect to the grammatical class. The variant $\mathrm{HMH}$ is the traditional form, and the variant $\mathrm{M}$ can be regarded as the most frequent form based on the findings of the lexical data. The variant $\mathrm{H}$ is analogous to the contact-cheek variant in ASL. As reported in Section 6.3.1, both the $\mathrm{HMH}$ and $\mathrm{M}$ variants are favoured in predicate adjectives, and compounds favour the $\mathrm{H}$ variant. While the following phonological environment is not correlated to the DEAF variants, it appears to be the case in HEARING (both the following segment and the following location). Yet, no effect of the Obligatory Contour Principle is found. There are two possible reasons why this study deviates from the study of ASL with respect to the Obligatory Contour Principle. First, language specific differences in how the variation was coded may have played a role: the focus of the current study was movement, while the focus in the ASL study was location. Second, and relatedly, the base form of the sign itself differed: different handshapes were used in the two languages.

For the nonlinguistic factors, the interaction between age and region was found to be significant in both the choice between citation and non-citation

\footnotetext{
${ }^{5}$ See Guy and Bobery (1997) for explanation of the Obligatory Contour Principle in studies of phonological variation.
} 
forms and between the two non-citation forms in the ASL study. Similarly, age and school in both DEAF and HEARING in HKSL have reached statistical significance in the cross-tabulation analysis.

In sum, although the methods adopted (multivariate analysis in the ASL study and distributional analysis in the current study) and the focus of the two studies are different, comparable results regarding linguistic and social factors are found. Both studies have demonstrated the important role of grammatical constraints in the variant choice of DEAF (also in HEARING for HKSL), though this should not be construed as meaning only grammatical category is a significant constraint (see Section 6.4.3). While age and region combined to form a strong factor group in ASL, age and school are strong effects in HKSL, in which school is analogous to region in the ASL study for reasons explained in Chapters 2 and 4.

\subsubsection{Language change in progress?}

In the ASL study, Lucas et al. (2001) proposed that "the grammatical constraints are a synchronic reflex of a change in progress that originates in compounds and then spreads to nouns and adjectives and finally to predicates. A change from ear-to-chin to chin-to-ear, beginning with compounds, a grammatical class that is most subject to change, is arguably a shift in the direction of greater ease of production" (p. 127). They further elaborated that this change correspond to "Kroch's (1978) model of change from below" (p. 127).

This may also be true in the case of DEAF in HKSL in view of the distribution pattern of compounds and predicate adjectives. Instead of a change 
from ear-to-chin to chin-to-ear, then contact-cheek as in ASL, I postulate one possible change of DEAF in HKSL would be from $\mathrm{HMH}$ to $\mathrm{MH}$ (holddeletion), then from $\mathrm{MH}$ to $\mathrm{H}$ (movement-deletion). There is no doubt that $\mathrm{HMH}$ is the underlying form of DEAF given the evidence from the HKSL publications (see Appendix D, Figure 46). Deletion is a common phonological process observed in both spoken and signed languages, which certainly tends to simplification. Although HKSL compounds which have DEAF as the first element are less lexicalized than ASL compounds, deletion is often found in the compounding processes and lexicalized compounds in other HKSL signs. Concerning the behaviour of signers from different age groups, both the younger and middle-aged groups favour the $\mathrm{H}$ variant, whereas the older group prefers the $\mathrm{HMH}$ form. In addition, the lexical data shows that the HKSL users are not quite aware of the forms $\mathrm{MH}$ and $\mathrm{H}$, but they are actually using them quite a lot in their conversation. This supports the idea that such a change is below the level of conscious awareness of the language users.

Moreover, I speculate there is another change going on. In the lexical data, the most frequent variant is $\mathrm{M}$. The form MMM is also produced by a considerable number of signers, just slightly fewer than the underlying $\mathrm{HMH}$ form. Therefore, these forms are the three main variants used by the participants. I propose another possible change of DEAF would be from $\mathrm{HMH}$ to MMM (hold-deletion and movement epenthesis), then from MMM to M (distalization, i.e., movement articulator shift from elbow to wrist). Indeed, the forms MMM and M look very much the same and I did group them together when I first coded the lexical data. It was the conversation data which offered more tokens from each informant that prompted me to separate the two forms. Nevertheless, MMM seems to 
be the transitional form and $\mathrm{M}$ appears to be the innovative new form. Compared with the traditional old form $\mathrm{HMH}$, this new form $\mathrm{M}$ looks simplified and more fluid in production with the initial and final holds deleted. Although movement is added, the entire movement occupies a smaller signing space, resulting in some displacement of location. This also entails the lost of iconicity of the original form, i.e., BAD in hearing and BAD in speaking (see Section 4.5.2.1).

Even though such a change seemingly resulted in an ease of articulation, I suspect this change is motivated by a redefining of signers' deaf identity. The origins of the innovative form is unknown, at least I do not know when and how the new form began to emerge. In fact, viewing the variants found in the current study has reminded me of a YouTube video made by a Chinese deaf woman I watched some time ago. This video was about the difference between calling someone "deaf-mute" and "deaf person" and she advocated the change of the sign DEAF in CSL. ${ }^{6}$ The form of DEAF in CSL is the same as the one in HKSL due to the historical relationship of the two languages mentioned in Chapter 3. The deaf woman in the video criticized use of the BAD-handshape because it has negative connotations and proposed replacing the pinky finger with the index finger. The proposed new form was the same as DEAF in ASL (see Figure 4.12a on page 98 for sign illustration). Apparently, such advocacy has not succeeded, but the video has been viewed over 330,000 times globally in the past 8 years. I do not know how many Hong Kong deaf people have

\footnotetext{
6The video title is “CSL: Deaf, not Dumb/ “壟啞”與“聾人”的區別" (link: www.youtube.com/watch? $\mathrm{v}=8 \mathrm{j} 4 \mathrm{bkQ} 09 \mathrm{Bbs})$. She first explained that the sign DEAF in CSL means DEAF and DUMB / MUTE and commented that it is wrong to call a deaf person "deaf and dumb/mute" in Chinese.
} 
watched that video and I do not mean that the change in HKSL DEAF is motivated by those who have watched the video. I just want to point out that an initiation of a change in such an essential lexical item can be originated from the increasing awareness of one's language, culture, and identity, and it is possible that some Hong Kong deaf people may have similar negative feeling about the sign.

Over the past decade or so, many things have happened in the Hong Kong deaf community. On the one hand, the closing down of deaf schools has had a great impact on the Hong Kong deaf community. Beginning in 2004, there was the conversion of HSD to a hearing mainstream school. In 2006 and 2007, respectively, VSD and CMS closed down. Since then, there has been only one deaf school (LSD) left in Hong Kong. These events have not only impacted on those who went to these schools, but the whole deaf community.

On the other hand, there has been an increase in deaf awareness and positive attitudes towards HKSL among deaf people and the general public due to several reasons, such as the establishment of the Centre for Sign Linguistics and Deaf Studies (CSLDS) in 2003 as explained in Chapter 3. In 2007, the issue of standardization of sign language was discussed in the Rehabilitation Programme Plan (2005-2007), which developed into a series of outputs promoting HKSL. Deaf people have been invited to take part in a TV programme launched in 2011, together with some celebrities. Despite the controversies of the programme from the deaf perspective, it has promoted an open attitude towards HKSL in the general public. In 2008, China ratified the Convention on the Rights of Persons with Disabilities (CRPD) which brought about the legitimation (together with some finan- 
cial support) of HKSL in deaf education. The growing sense of deaf pride is evidenced in the launching of the Hong Kong Deaf Festival in 2009 and the Hong Kong International Deaf Film Festival in 2010.

All the above mentioned events (and many more not mentioned here) may have induced and bolstered the dissemination of the innovative new form of DEAF, which the younger signers find better represents them. In short, the fundamental cause of such a change is "the human instinct to establish and maintain social identity" (Chambers, 2009, p. 266).

Lastly, it should be noted that there is a discrepancy between the distribution patterns of DEAF and HEARING. This discrepancy may suggest a different stage of change in the two variables. Since the sign DEAF is central to the identity of the participants and the sign HEARING denotes 'others,' all those non-deaf people, it is not surprising that the change of HEARING would follow that of DEAF and not the other way round.

\subsubsection{Potential implications}

I understand it may be a bold proposition that there are two different changes going on in the DEAF/HEARING variables, based on just a small and somewhat skewed dataset and some contingency tables. Yet, this is the possibility that I observe from my limited data and analysis. Certainly, more work needs to be done in order to support such a claim, and variation and change in the sign DEAF deserve further investigation for its importance to the deaf community.

Another point to note is the anatomical difference between DEAF and 
HEARING. For example, the HMH form of DEAF is made with the palm facing the signer, while the palm orientation of the HEARING one is always to the side. The bodily constraints of the two signs are different and hence, may affect the behaviour of their variants and their interaction with the immediate phonological environment. This may be a possible explanation for the different distribution patterns observed in the conversation data.

In Section 6.3.1, a caveat on the findings about the effect of the following segment and location in HEARING was made because of the small number of pauses and possible relation with the target sign location. In spite of that, the results suggest the probable influence of the following phonological environment. It is possible that the movement coding was not enough to capture the possible relation between the variants and the movement of the preceding/following segment. In this analysis, the movement of the immediate phonological environments were coded as simple, complex, or no movement. More detailed movement coding may yield a different outcome. Like many other studies looking at the immediate phonological environment, only one parameter was coded and tested at a time. The possibility of combined parameters as factor groups has not been explored. Furthermore, as remarked in the ASL study, although grammatical function is the strongest effect, it is possible that "unexplored phonological factors may play a role in the patterning of grammatical constraints" (Lucas et al., 2001, p. 146).

Last but not least, although the data suggests that there is a change in progress, it does not mean that the traditional form of DEAF and HEARING will be replaced by the new form completely in the future. They may turn out to be stable variables. Like other sign language communities, change 
in HKSL is happening much faster than its surrounding spoken languages. Future research in the language may reveal a different picture.

\subsection{Chapter summary}

In this chapter, I began by presenting the results of the lexical data. The two signs DEAF and HEARING collected through the lexical elicitation task have demonstrated a similar distribution pattern across the five variants. The variant choice of both signs seems to associate with the school, age, and gender as revealed in the cross tabulation analysis. I then reported the results of the conversation data. The analysis of the conversation data suggests that the two variables are conditioned by both linguistic and nonlinguistic factors, though with distinct distribution patterns. For the linguistic factors, grammatical constraints are the most robust and this parallels the findings of the ASL study. For the social factors, both school and age are strong effects in governing variant choice in both DEAF and HEARING, a result which also echoes that of the ASL study. While the following phonological environment may have an effect on HEARING and gender appears to be a significant factor in conditioning the choice of two variants in HEARING, neither of these factors has an effect on DEAF.

With regard to language change, I proposed there are two different kinds of change observed in the variables. The first type originated in compounds, whereby the traditional $\mathrm{HMH}$ form changes to $\mathrm{MH}$ after holddeletion, and then to the simplest form $\mathrm{H}$ after movement-deletion. The second is motivated by signers redefining their deaf identity. In this change, the initial and final holds in the traditional $\mathrm{HMH}$ form are deleted and 
movements are added, resulting in the MMM transitional form; the movement articulator is then distalized from elbow to wrist, ending with the $\mathrm{M}$ variant. The new form has obscured the negative connotations associated with the traditional form. Finally, the potential implications of analyzing DEAF/ HEARING have been discussed.

In the next chapter, I will turn to the second phonological variable: location drop, a variable that has been investigated in ASL, Auslan, and NZSL. 



\section{Chapter 7}

\section{Phonological variation 2: location drop}

\subsection{Overview}

In this chapter, I first present the findings of the second phonological variable - the location variable in the class of signs articulated at or near the forehead area, such as THINK, which may be lowered in everyday conversation. Figure 7.1 illustrates the citation form and the non-citation form of THINK. Results of the linguistic factors and the social factors are reported in Sections 7.2.1 and 7.2.2 respectively. Section 7.3 is a discussion section. I compare the findings of the present study with previous studies on ASL, Auslan, and NZSL in Section 7.3.1. Whether this variation reveals a language change in progress is considered in Section 7.3.2. The unique findings in this study that link with the deaf school name signs and deaf related work environment are described in Sections 7.3.3 and 7.3.4. Finally, 
the chapter summary in Section 7.4 summarises the results.

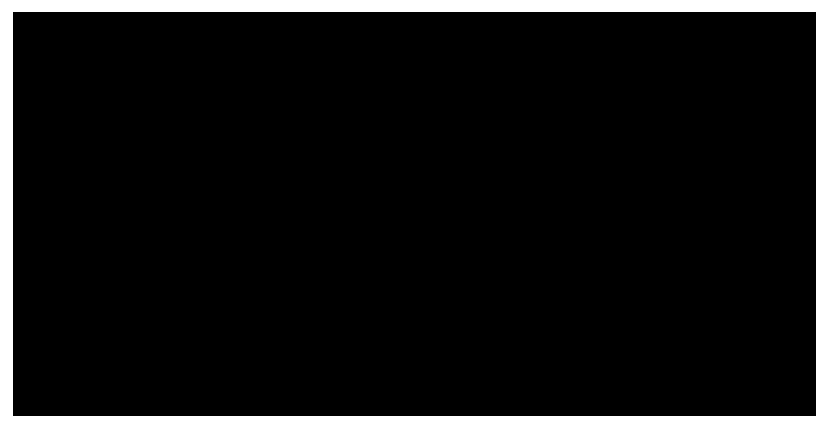

Figure 7.1: Citation form and non-citation form of THINK

\subsection{Results}

The non-citation forms of the target signs account for $23.4 \%(\mathrm{~N}=187)$ of all 800 tokens. The complete list of the target signs represented by 75 lexical types is given in Section 4.3 on page 110. The occurrence of non-citation forms is far less common compared with the ASL, Auslan, and NZSL data, in which non-citation forms represent $53 \%, 45 \%$, and $43 \%$ respectively. It is interesting to note the relation between the age of the language and the percentage of non-citation forms. The older the language, the more occurrences of non-citation form, i.e., more location dropping in the class of signs produced at or near the forehead. If we take the establishment of the first deaf school as a reference point for the formation of a local variety of a sign language, ASL would be near 200 years old, Auslan would be around 155 years old, ${ }^{1}$ NZSL would be about 135 years old, and HKSL

\footnotetext{
${ }^{1}$ In fact, both ASL and Auslan have a much longer history and linkages to other preexisting sign languages (Frishberg, 1975; Johnston, 2003). Because of the historical link between BSL and Auslan, the appropriate 'age' for Auslan is debatable.
} 
would be barely 80 years old.

\subsubsection{Linguistic factors}

Cross-tabulation and early analysis of the data revealed that change in hand dominance, markedness of handshape, and lexical frequency do not show any effect on the choice between citation and non-citation forms of the location variable. It was necessary to exclude two kinds of tokens before the multiple regression analyses were conducted in Rbrul. First, categorical users of one variant or another (in this case, two signers who only used citation forms) were excluded from the analysis of variation, since obviously they do not vary in respect of location drop. Second, when a signer switches his/her hand immediately before or after (or sometimes both) the production of the target sign, it is not appropriate to code for 'location/contact of preceding/following sign' as in this coding refers to location of preceding/following sign when the signer is using the same hand. Therefore, 40 observations from two knockouts and 65 observations of the target signs involving switches in hand dominance were excluded. This resulted in 695 tokens for final analysis.

Table 7.1 shows the significant factor groups and their corresponding results in an Rbrul analysis. Three linguistic factor groups were significant at the 0.05 level. The strongest linguistic constraint was the grammatical class $x$ deaf school name sign group, with a range value of 48 . Within this factor group, the name signs of deaf school highly favoured non-citation form (with a factor weight of 0.828$)$, followed by verbs (0.585) and grammatical items (0.532). On the contrary, proper nouns other than deaf school dis- 
favoured non-citation form the most, with a factor weight of 0.344 . However, this result should be interpreted with caution for there were only seven tokens of other proper nouns. ${ }^{2}$ The other two significant linguistic factors related to the preceding phonological environment: preceding segment and preceding location. When the target sign followed a pause, it favoured non-citation forms (0.6); when it followed a sign, it favoured citation forms (0.4). While the preceding signs in the body area favoured noncitation forms (0.563), those in the head region favoured citation forms (0.437). Whether or not the preceding sign made contact with the body was not significant, and nor were any factors involving the following phonological environment. The results suggest that there may be anticipatory (regressive) assimilation between the target sign and the preceding segment. If the preceding segment is a pause, during which both hands are at rest, the target sign is more likely to be lowered at a non-citation location. Similarly, if the preceding sign is produced at the level of the body, the target sign tends to be produced below the forehead, i.e., non-citation location.

\footnotetext{
${ }^{2}$ This small number of tokens may be the cause of the higher percentage of noncitation forms in this category (14\%) than adverbs $(12 \%)$ and nouns $(12 \%)$, while the latter two have greater factor weights ( 0.377 and 0.346 respectively).
} 


\begin{tabular}{|c|c|c|c|c|}
\hline \multicolumn{5}{|l|}{$\begin{array}{l}\text { Application value: non-citation form } \\
\text { Input probability: } 0.187 \\
\text { Deviance: } 660.448 \text { (log likelihood: -1320.896) }\end{array}$} \\
\hline & Log odds & Weight & $\%$ & $\mathrm{~N}$ \\
\hline \multicolumn{5}{|l|}{ Age.Work.Education.School $(\mathrm{p}<0.0001)$} \\
\hline Younger.Non-deafWork.Average/aboveEdu.LSD & 1.451 & 0.81 & 56 & 39 \\
\hline Middle.Non-deafWork.Average/aboveEdu.LSD & 1.242 & 0.776 & 45 & 67 \\
\hline Younger.Non-deafWork.Average/aboveEdu.CMS+ & 0.582 & 0.642 & 28 & 88 \\
\hline Middle.Non-deafWork.Average/aboveEdu.CMS+ & 0.568 & 0.638 & 26 & 72 \\
\hline Older.Non-deafWork.LowEdu.HSD+ & 0.291 & 0.572 & 29 & 17 \\
\hline Middle.Non-deafWork.Average/aboveEdu.HSD+ & 0.266 & 0.566 & 24 & 54 \\
\hline Younger.DeafWork.Average/aboveEdu.HSD+ & 0.149 & 0.537 & 20 & 76 \\
\hline Older.Non-deafWork.Average/aboveEdu.LSD & 0.054 & 0.513 & 18 & 38 \\
\hline Younger.DeafWork.Average/aboveEdu.LSD & -0.046 & 0.489 & 20 & 20 \\
\hline Older.Non-deafWork.LowEdu.OMS+ & -0.154 & 0.462 & 16 & 149 \\
\hline Older.Non-deafWork.Average/aboveEdu.OMS+ & -1.186 & 0.234 & 6 & 36 \\
\hline Older.DeafWork.Average/aboveEdu.OMS+ & -1.499 & 0.183 & 5 & 20 \\
\hline Older.Non-deafWork.Average/aboveEdu.HSD+ & -1.718 & 0.152 & 5 & 19 \\
\hline Range & & 66 & & \\
\hline \multicolumn{5}{|l|}{ Grammatical Class.Deaf School $(\mathrm{p}<0.0001)$} \\
\hline Deaf school name sign & 1.571 & 0.828 & 54 & 50 \\
\hline Verb & 0.342 & 0.585 & 26 & 379 \\
\hline Grammatical item & 0.129 & 0.532 & 27 & 15 \\
\hline Adjective & -0.258 & 0.436 & 18 & 112 \\
\hline Adverb & -0.501 & 0.377 & 12 & 67 \\
\hline Noun & -0.637 & 0.346 & 12 & 65 \\
\hline Other proper noun & -0.646 & 0.344 & 14 & 7 \\
\hline Range & & 48 & & \\
\hline \multicolumn{5}{|l|}{ Preceding Segment $(\mathrm{p}<0.01)$} \\
\hline Pause & 0.404 & 0.6 & 48 & 65 \\
\hline Sign & -0.404 & 0.4 & 22 & 630 \\
\hline Range & & 20 & & \\
\hline \multicolumn{5}{|l|}{ Preceding Location $(\mathrm{p}<0.05)$} \\
\hline Body & 0.252 & 0.563 & 28 & 461 \\
\hline Head & -0.252 & 0.437 & 17 & 234 \\
\hline Range & & 13 & & \\
\hline $\operatorname{Total}(\mathrm{N})$ & & & & 695 \\
\hline
\end{tabular}

Table 7.1: Results of Rbrul analysis of significant factor groups

Since forehead-located deaf school name signs (proper nouns) may only exist in the current study (no such signs were described in the ASL, Auslan, and NZSL data) and the results showed that deaf school name sign ranks the highest within the grammatical category, I conducted another 
analysis after excluding the deaf school name signs in order to obtain a more comparable dataset for cross-linguistic comparison. The new dataset consisted of 645 observations. Considering the small number of tokens of proper nouns after removing deaf schools, other proper nouns were grouped together with nouns. The significant factor groups and their corresponding results in the Rbrul analysis are shown in Table 7.2. The three significant linguistic factors remained the same, but their relative strength varied. Without the deaf school name signs in the dataset, the range of grammatical class dropped from 48 to 24 . While verbs and grammatical items favoured non-citation form, with factor weights of 0.611 and 0.607 respectively; adverbs and nouns (including proper nouns) disfavoured it (0.418 and 0.375 respectively). Adjectives appeared to be rather neutral (0.489). With the same range value of 24 , the preceding segment, together with the grammatical class, were the strongest linguistic constraints. Similar to the previous model, preceding pause and preceding location in the body region strongly favoured non-citation form (0.621 and 0.577 respectively). 


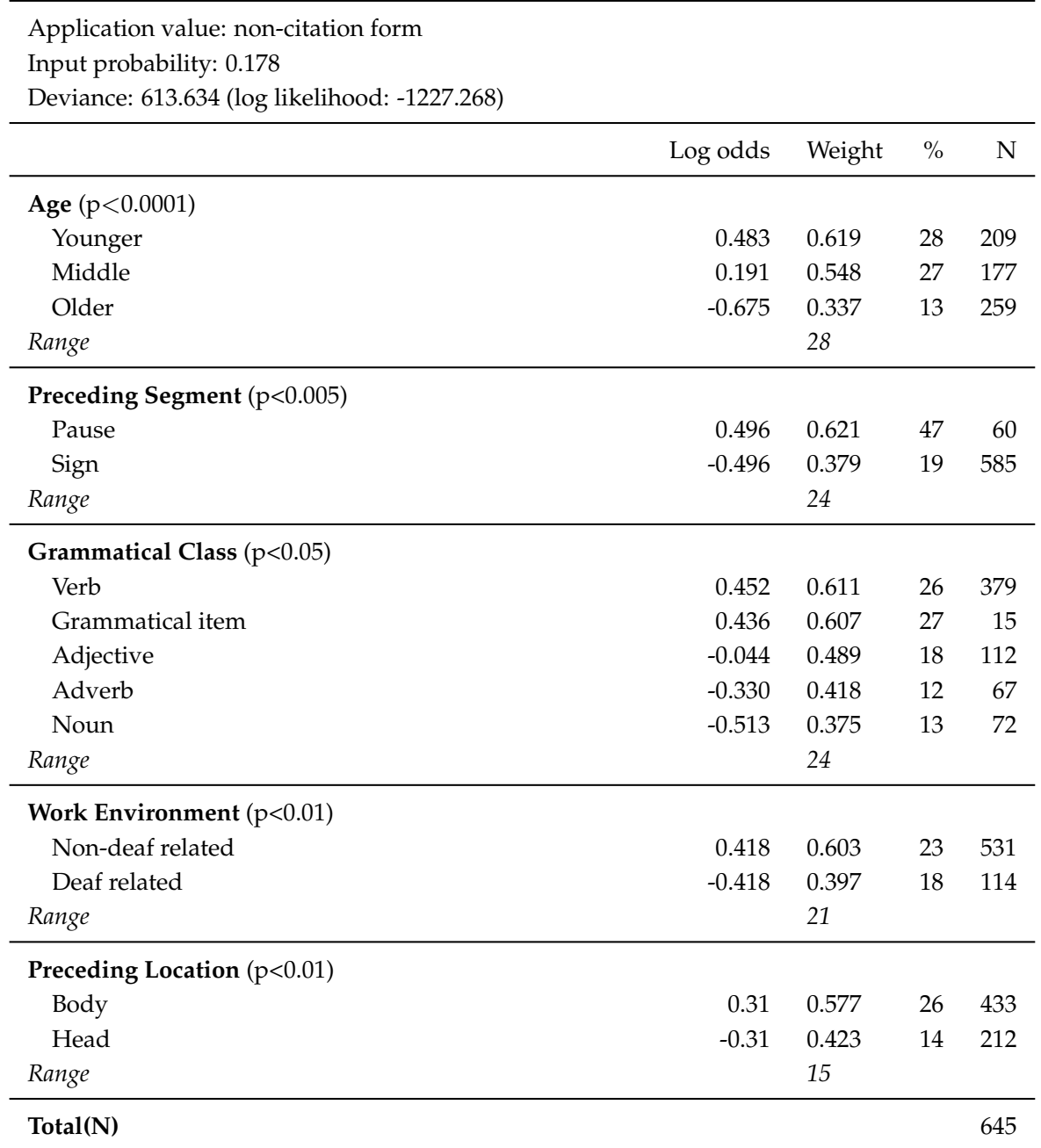

Table 7.2: Results of Rbrul analysis of significant factor groups (excluded deaf school name signs)

\subsubsection{Social factors}

As stated earlier, many social factors are related to each other. Initial analysis showed that they interact with each other as well. As shown in Table 7.1, four social factors: age, work environment, education, and school, interacted with each other and formed the strongest factor group (with a range 
value of 66). In general, older signers (aged 50 and above) disfavour noncitation form, except those who had attended HSD and with lower educational level (0.572). There is virtually no effect for older participants who went to LSD (0.513). Signers who had attended OMS disfavour noncitation form (factor weights between 0.183 and 0.462 ), while those who went to other schools favour it (between 0.537 and 0.81 ). The only exception is the older signer with average or above education who had studied in HSD. This signer contrasts with the previous mentioned signer with similar background except for educational level. The older signer with higher education strongly disfavours non-citation form (0.152), whereas the one with lower education favours it (0.572). This parallels the less educated signers from OMS with a relatively high factor weight of 0.462 when compared with the more educated OMS signers (0.234) and one of those who works in a deaf-related environment with a very low factor weight of 0.183 . This reflects that both education and work environment have constrained the use of non-citation form. Additionally, younger signers from LSD favour non-citation form the most (0.81), except the one who works in a deaf-related organization, who is more neutral or slightly disfavours it with a factor weight of 0.489 . On the other hand, all younger signers who had attended HSD are working in deaf-related settings. They still favour non-citation form, like those who do not have a deaf-related job (0.566 and 0.572), but with a comparatively lower factor weight of 0.537 .

With respect to the analysis in Table 7.2 using the dataset without deaf school name signs, only two social factors: age and work environment remained significant. Age was proved to be the strongest constraint among all factor groups with a range value of 28. Within this factor group, both younger and middle-aged signers favour non-citation form (0.619 and 0.548 
respectively), while older signers strongly disfavour it (0.337). Work environment was also found to be significant at the level of $\mathrm{p}<0.01$, though with a weaker constraint (range=21). Signers who are working in a deaf-related role disfavour non-citation form (0.397), and their counterparts favour it (0.603). The school factor group was not significant in this model. It may not be surprising when we look at the breakdown of the deaf school name signs produced by signers from different schools. As displayed in Table $7.3,14$ out of $18(78 \%)$ of the total tokens of LSD were non-citation forms and out of these 14 tokens, 12 were produced by the signers from LSD. Seven out of the ten tokens of HIU-CHONG (one of the OMS) were made by those who had studied in OMS, and its non-citation form accounted for only $11 \%$. This showed that the production of these name signs was strongly related to the school background of signers. Therefore, the exclusion of these target signs has resulted in the loss of significance of the school factor group. Another social factor that failed to show significance in this model was education. This may due to the fact that most of the signers with low education are from OMS.

\begin{tabular}{lccccccccccc} 
& \multicolumn{2}{c}{ CMS+ } & \multicolumn{2}{c}{ HSD +} & \multicolumn{2}{c}{ LSD } & \multicolumn{2}{c}{ OMS+ } & \multicolumn{2}{c}{ total } \\
\hline Target Sign & $\#(\mathrm{~N})$ & $\%$ & $\#(\mathrm{~N})$ & $\%$ & $\#(\mathrm{~N})$ & $\%$ & $\#(\mathrm{~N})$ & $\%$ & $\#(\mathrm{~N})$ & $\%$ \\
\hline HIU-CHONG & - & - & $0(3)$ & 0 & - & - & $1(7)$ & 14 & $1(10)$ & 11 \\
HSD & $4(6)$ & 67 & $4(9)$ & 44 & $2(3)$ & 67 & $2(4)$ & 50 & $12(22)$ & 55 \\
LSD & $2(5)$ & 40 & - & - & $12(13)$ & 92 & - & - & $14(18)$ & 78
\end{tabular}

Table 7.3: Number and percentage of non-citation form, school by target signs (deaf school name signs)

However, a quite different picture is revealed when we look at the conditional inference trees constructed for the location drop data. Figures 7.2 and 7.3 illustrate the trees using age as continuous and discrete factors respectively. We can see from the two figures that age is influential on the 
choice between citation form and non-citation form only when it is considered as a continuous factor. Furthermore, this age constraint is only conditioning the male signers from CMS and LSD, with those aged over 38 disfavour non-citation forms. For those aged 38 and below, the LSD men use significantly more non-citation forms than the CMS men. When age is treated as a discrete factor, only school and gender are predictors of location drop. It is interestingly to see that gender is the second most important social factor as shown in the conditional inference trees, but it is not significant in the multivariate analysis. As demonstrated in the trees, women from CMS and LSD produced considerably more citation forms than their male counterparts.

When I further constructed the conditional inference trees with the deaf school name signs excluded, age became the most important factor if treated as continuous (Figure 7.4), and school is still the strongest constraint if considered as discrete (Figure 7.5). Again, gender plays a role in both trees, but it is conditioning the LSD signers only when age is a continuous factor. For LSD signers aged 38 and below, men highly favour non-citation forms as opposed to women. 


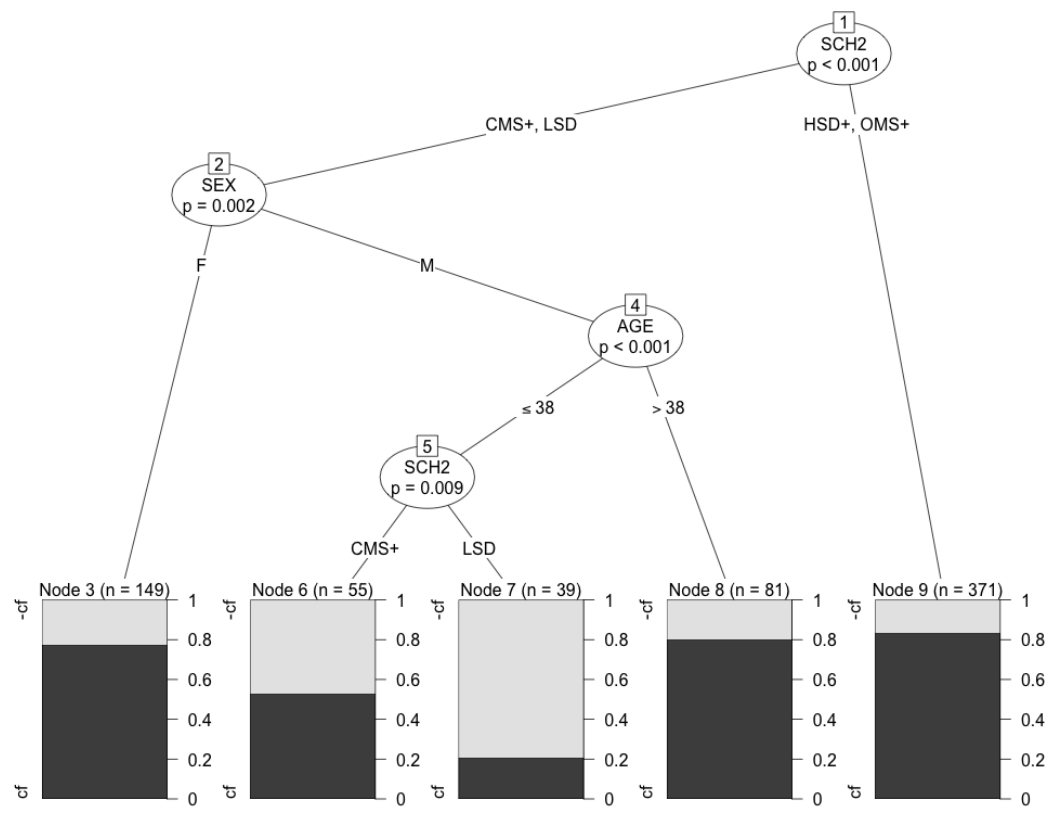

Figure 7.2: Conditional inference tree for location drop (age as continuous) ( $\mathrm{cf}=\mathrm{citation}$ form; - $\mathrm{cf}=$ non-citation form)

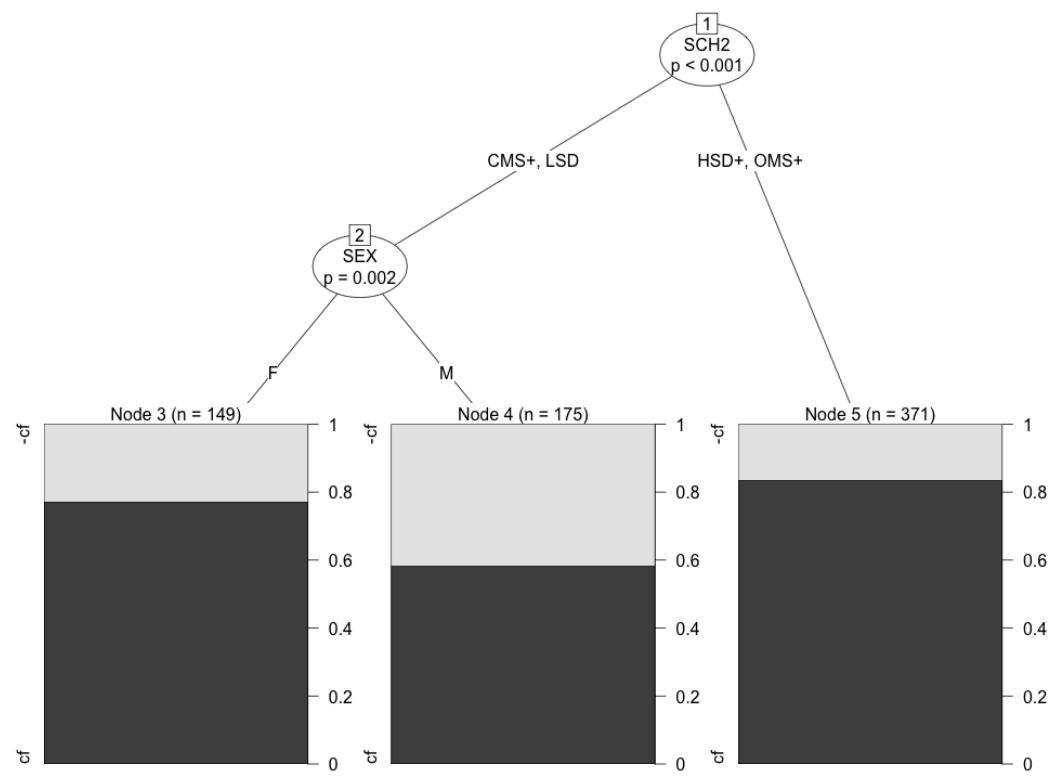

Figure 7.3: Conditional inference tree for location drop (age as group) ( $\mathrm{cf}=\mathrm{citation}$ form; -cf=non-citation form) 


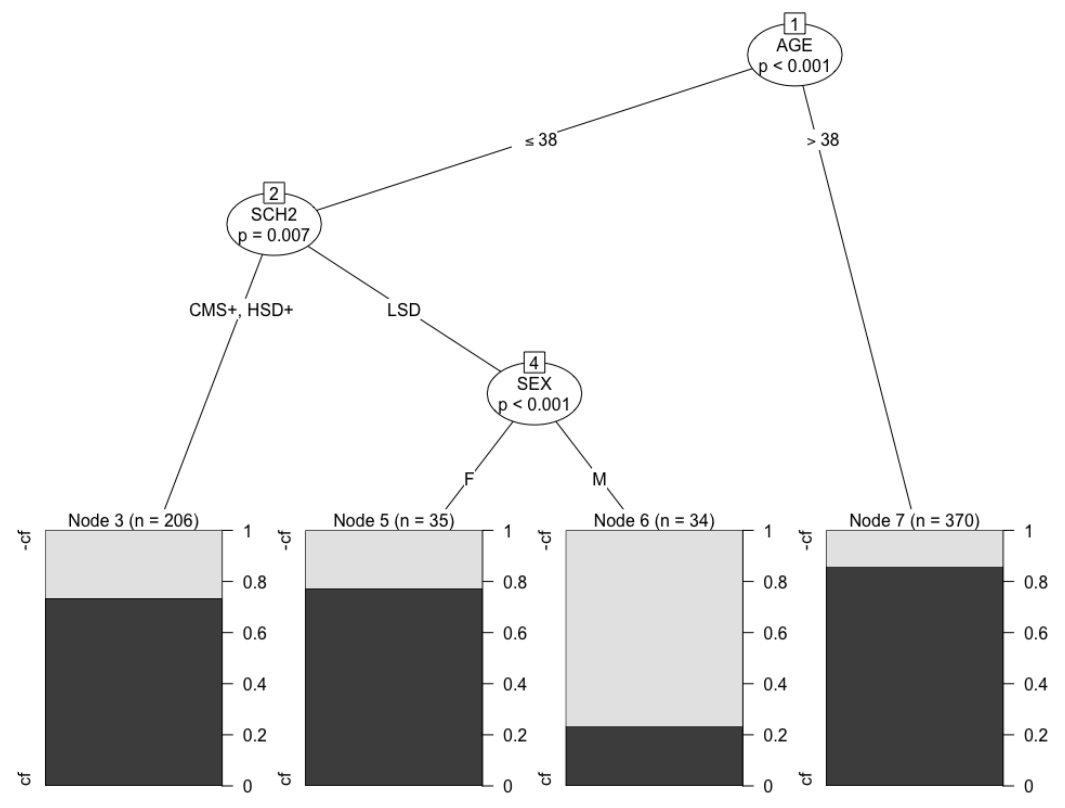

Figure 7.4: Conditional inference tree for location drop (excluded deaf school name signs and age as continuous) ( $\mathrm{cf}=\mathrm{citation}$ form; $-\mathrm{cf}=$ non-citation form)

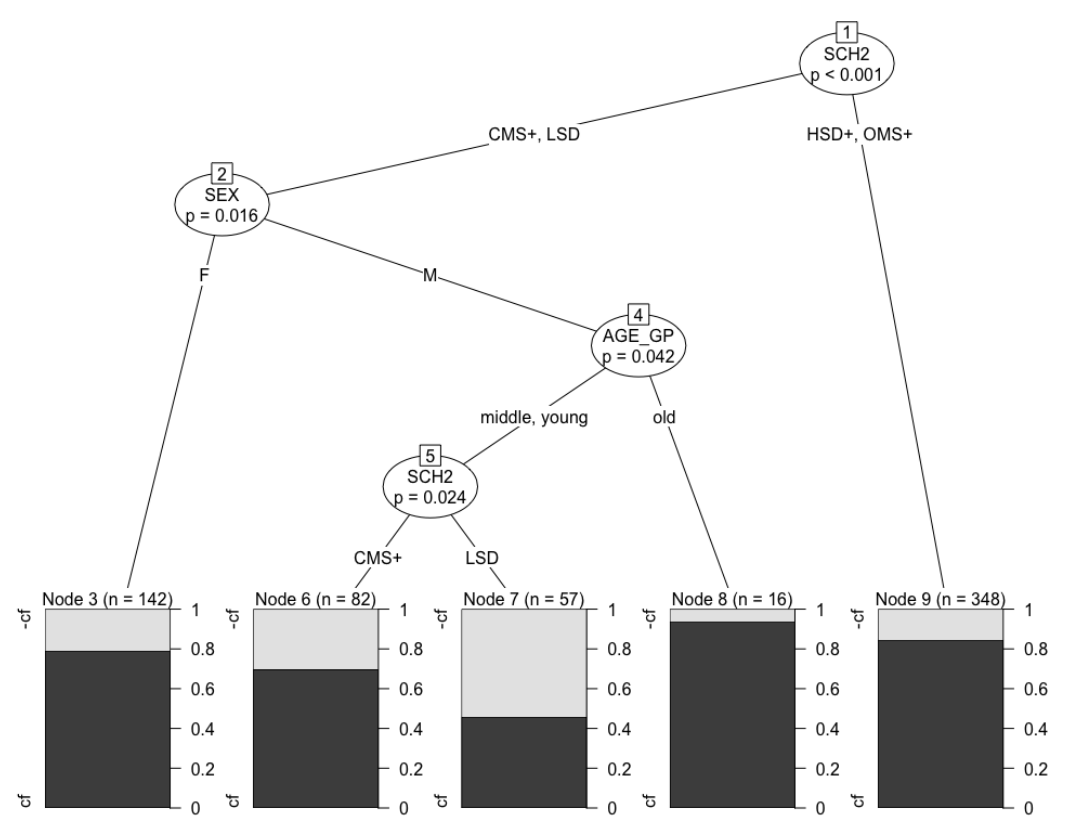

Figure 7.5: Conditional inference tree for location drop (excluded deaf school name signs and age as group) ( $\mathrm{cf}=\mathrm{citation}$ form; $-\mathrm{cf}=$ non-citation form) 
In sum, the results of the Rbrul analysis and the conditional inference trees appear to be quite different. The Rbrul analysis demonstrates an interaction between four social factors: age, work environment, educational, and school. Older signers, which included all those who had attended OMS, disfavour the lowering of target signs. Among all schools, signers from LSD favour non-citation form the most, unless they are older (aged 50 and above) or have a deaf-related job. HSD signers favour non-citation form, except the person who is older and has average or above educational attainment. The conditional inference tree analysis reveals that gender is also an influential factor, in addition to age and school. While HSD and OMS prefer citation forms, male signers from CMS and LSD highly favour non-citation forms, particularly LSD men aged 38 and below.

When the deaf school name signs were removed from the data, only age and work environment remained to be significant social factors in the Rbrul analysis. Older signers and those work in a deaf-related environment disfavour non-citation forms. On the other hand, in the conditional inference trees, school remains a strong factor when age is treated as discrete groups and, age became the most important predictor when considered as continuous. Gender is still a strong effect in both conditions.

\subsection{Discussion}

The results of the multivariate analysis show that location drop in HKSL, like ASL, Auslan, and NZSL, is not random, but is constrained by various linguistic and social factors in a systematic way. Nonetheless, the findings of the four studies have both similarities and dissimilarities in terms of 
linguistic and social constraints. In this section, I will first compare the results of all four studies. Then, I will consider the possibility of language change in progress in HKSL with regard to the location drop in the class of signs under examination. Lastly, I will discuss the unique findings of the current study, i.e., the effects of deaf school name signs and signers' work environment.

\subsubsection{Comparison with ASL, Auslan, and NZSL results}

Concerning phonological factors, all four studies (Lucas et al., 2001; Schembri et al., 2009) have investigated the preceding and following segments (sign or pause), preceding and following location (head or body), and preceding and following contact (no contact or contact with head/hand/body). The only factor that demonstrated significance in all four sign languages is preceding location. The results for linguistic factors across HKSL, ASL, Auslan, and NZSL are shown in Table 7.4. In all four languages, target signs following a sign produced at the head are more likely to be produced at the forehead, i.e., the citation form location. By contrast, if the preceding sign is produced below the head, the target sign tends to be lowered. When we compare the strength of this phonological factor (preceding location) across the four languages, the strength in ASL is relatively weak with a range value of 5 , while its effect in the other three languages is much stronger (range value between 13 and 17). This may suggest a different stage in the developmental path of the location variable for the different languages.

Taking sound change in spoken languages as a reference, the rate of sound change is very often observed to follow an S-shaped curve when 
plotting the cumulative frequency of binomial distribution (Labov, 1994). In discussing the sound changes in Philadelphia, Labov (1994) reconstructed five stages of change by combining evidence from real-time and apparenttime data. These five stages of development are: (1) incipient, (2) new and vigorous, (3) midrange, (4) nearly completed, and (5) completed (pp. 7983). Nevalainen and Raumolin-Brunberg (2003) further applied these five stages in terms of the proportion of new forms as in Table 7.5 in analysing innovative forms in the history of English.

If phonological changes in signed languages, such as location drop here, follow a similar pattern as sound changes in spoken languages, the change in HKSL can probably be classified as 'new and vigorous,' while the changes in the other three languages are perhaps in the mid-range.

For another phonological factor suggesting assimilation, a different pattern is observed. Preceding pause is found to be important in HKSL for inducing non-citation forms, and a following pause has effects in Auslan and NZSL, but neither one is significant in the ASL data. For HKSL, whether the preceding segment is a pause or a sign is as strong a constraint as the grammatical category (both range value 24), and these are the strongest constraints in HKSL. However, preceding pause failed to reach significance in ASL, Auslan, and NZSL, and the grammatical function (together with lexical frequency in Auslan and NZSL) is the strongest constraint across these three languages (range values of 27, 19, and 28 respectively). Preceding pause as a strong and significant factor may indicate an early stage of location variation when considering the comparatively low input probability in HKSL and the young age of this language. 


\begin{tabular}{|c|c|c|c|c|}
\hline $\begin{array}{r}\text { Input probability } \\
\text { non-citation form percentage }\end{array}$ & $\begin{array}{l}\text { HKSL }(\mathrm{N}=645) \\
0.178 \\
22\end{array}$ & $\begin{array}{l}\text { ASL }(\mathrm{N}=2594) \\
0.518 \\
53\end{array}$ & $\begin{array}{l}\text { Auslan }(\mathrm{N}=2667) \\
0.427 \\
45\end{array}$ & $\begin{array}{l}\text { NZSL }(N=2096) \\
0.412 \\
43\end{array}$ \\
\hline \multicolumn{5}{|l|}{ Grammatical Class (x Freq) } \\
\hline Verb & 0.611 & 0.581 (prep/int) & 0.577 (HF verb) & 0.566 (HF verb) \\
\hline Grammatical item & 0.607 & 0.486 (noun/verb) & 0.388 (others) & 0.499 (LF verb) \\
\hline Adjective & 0.489 & 0.316 (adj) & & 0.433 (LF noun/adj) \\
\hline Adverb & 0.418 & & & 0.291 (HF noun/adj) \\
\hline Noun & 0.375 & & & \\
\hline Range & 24 & 27 & 19 & 28 \\
\hline \multicolumn{5}{|l|}{ Preceding Segment } \\
\hline Pause & 0.621 & & & \\
\hline Sign & 0.379 & n.s. & n.s. & n.s. \\
\hline Range & 24 & & & \\
\hline \multicolumn{5}{|l|}{ Preceding Location } \\
\hline Body & 0.577 & 0.514 & 0.543 & 0.518 \\
\hline Head & 0.423 & 0.463 & 0.369 & 0.390 \\
\hline Range & 15 & 5 & 17 & 13 \\
\hline \multicolumn{5}{|l|}{ Preceding Contact } \\
\hline No contact & & & 0.509 & 0.516 \\
\hline Contact & n.s. & n.s. & 0.537 (head/hand) & 0.481 \\
\hline & & & 0.448 (body) & \\
\hline Range & & & 9 & 4 \\
\hline \multicolumn{5}{|l|}{ Following Segment } \\
\hline Pause & & & 0.644 & 0.684 \\
\hline Sign & n.s. & n.s. & 0.480 & 0.483 \\
\hline Range & & & 16 & 20 \\
\hline \multicolumn{5}{|l|}{ Following Location } \\
\hline Body & & & 0.526 & 0.534 \\
\hline Head & n.s. & n.s. & 0.441 & 0.393 \\
\hline Range & & & 9 & 14 \\
\hline \multicolumn{5}{|l|}{ Following Contact } \\
\hline No contact & & 0.525 & & \\
\hline Contact & n.s. & 0.466 & n.s. & n.s. \\
\hline Range & & 6 & & \\
\hline
\end{tabular}

Table 7.4: Comparison of seven possible linguistic factors on location drop in HKSL, ASL, Auslan, and NZSL (application value: non-citation form)

\begin{tabular}{ll}
\hline Stage & Percentage of new forms \\
\hline Incipient & below $15 \%$ \\
New and vigorous & $15 \%$ to $35 \%$ \\
Mid-range & $36 \%$ to $65 \%$ \\
Nearing completion & $66 \%$ to $85 \%$ \\
Completed & over $85 \%$ \\
\hline
\end{tabular}

Source: Nevalainen and Raumolin-Brunberg (2003, p. 55)

Table 7.5: Percentage of new forms at different stages of sound change 
As Schembri et al. (2009) noted “... both beginning or resuming motion would involve overcoming the inertia of the hand, for example, and that this may have similar effects on location variation (i.e., physiological principles of economy of effort would predict that non-citation forms of signs may be more common after a pause or hold)" (p. 214). This triggering force in 'pulling the coming sign (i.e., the target sign) down' is apparently strong and is physically driven. Therefore, this factor may not be purely linguistic. While this 'semi-linguistic' factor may signify an early stage of the variable development, it may also be a factor unique to HKSL (and possibly other languages in the same language family, like Chinese Sign Language (CSL) or specifically the Shanghai variety). Thus far, we only have data from four languages. Similar research on languages such as CSL, ${ }^{3}$ British Sign Language (BSL, an older language related to Auslan and NZSL), Thai Sign Language (a younger language related to ASL), and Singapore Sign Language (a younger language related to both ASL and Shanghai Sign Language) $)^{5}$ would certainly offer us a better understanding of this classic variable in signed languages.

With respect to preceding and following contact, preceding contact is shown to be influential in Auslan and NZSL, following contact in ASL, but neither of them has effects on HKSL. No contact with any parts of the body

\footnotetext{
${ }^{3}$ The first deaf school in China was established in 1887 . So CSL would probably be nearly 130 years old.

${ }^{4}$ The first deaf school in Thailand was established in 1951 (Suvannus, 1987).

${ }^{5}$ The first deaf school in Singapore was established in 1952. Both ASL and Shanghai Sign Language were used in teaching the deaf in the past, and Signing Exact English 2 (a manually coded English sign system commonly known as SEE2) has dominated since the early 1980s (Phua, 2003).
} 
in the adjacent sign tends to slightly favour non-citation forms in the ASL data (with a factor weight of 0.525), but it is rather neutral in the Auslan (0.509) and NZSL (0.516) data. The Auslan data further illustrates that preceding contact with the head or hand favour non-citation forms (0.537) and those that involve contact with the body disfavour it (0.448). In view of this somewhat obscure difference, Schembri et al. (2009) pointed out the lack of coding for contactness in the target signs as a weakness in the studies. They speculated dissimilation may be at work with regard to the preceding or following contact, but the lack of information about whether actual contact happened in the target signs hinders further analysis.

Although I could have followed the lead of the previous studies in coding for contactness of the target signs in my data, I decided not to do so for the reason that determining the presence of contact is very challenging. Due to the limitation of two-dimensional video and human eyes, I found it difficult to code with certainty for contact of the preceding and following signs, especially those articulated at the head or face, where filming angle is extremely important and no cues from clothing can be traced (e.g., a small change on clothing can be observed if contact is made on the chest). Even with the high-definition (HD) filming technology, the quality of the videos did not help much in identifying such subtle variance. The new technology of optoelectronic motion capture systems that can capture and analyze three-dimensional signals may be helpful (Tyrone \& Mauk, 2010), but having devices on a signer's head and hand may hinder natural and free conversation. As far as we can tell here, the importance of preceding and following contact is rather low compared with other factors. The range values of the preceding contact in Auslan and NZSL are 9 and 4 respectively, and the one of the following contact in ASL is 6. This indicates a weak con- 
straint in all three languages.

The present study bolsters the claim that "the specific details of phonological variation differ from one signed language to the next, as is true of spoken languages" (Schembri et al., 2009, p. 221) and proposes that the inconsistent influence of linguistic factors on location variation may reflect the different developmental stage of the variable. Furthermore, the different methods in coding and analyzing data in the four studies may contribute to the different findings to some degree. As for coding, in HKSL, Auslan, and NZSL, only signs produced in citation form at or in proximity to the signer's forehead were coded, i.e., a more homogeneous set in terms of location; whereas the target signs in the ASL research were not so homogeneous, for they included signs with a lower location in citation form and lexicalized compound involving a component made below the forehead. In ASL, Auslan, and NZSL, the preceding location and contact were coded as NA if the target sign is preceded by a pause. Likewise, the following location and contact were coded as NA if the target sign is followed by a pause. In the current study of HKSL, due to the small number of tokens and the fact that Rbrul cannot deal with NA data, the preceding and following location were coded as 'body' in the case of pause, as both hands were in their resting position; the preceding and following contact were coded as 'no contact' when involving a pause. Lastly, change in hand dominance was coded in the HKSL, Auslan, and NZSL data; tokens involving a switch in hand dominance between target signs and adjacent signs were coded as NA in the current research, but not in the other two studies.

Regarding social factors, the results across HKSL, ASL, Auslan, and NZSL are shown in Table 7.6. Age proved to be a significant factor in 
HKSL, ASL, and Auslan; it is the strongest social constraint in HKSL and Auslan (range values of 28 and 15 respectively) and the second strongest in ASL (19). Although age is not significant in NZSL, its results showed a similar pattern. It is found that in all four communities, the younger signers favour the non-citation form whereas older signers disfavour it. This different preference between the younger and older generations as an indication of language change in progress will be discussed in the next section. While gender and region are important social factors in ASL, Auslan, and NZSL, the effect of gender fails to reach significance and region is not tested in the HKSL data. A different gender effect is observed in ASL as compared to that of Auslan and NZSL. Applying Labovian Principles, Schembri et al. (2009) suggested that women's preference for the citation form in ASL may indicate a change from above in the American deaf community. Conversely, women's tendency to use more innovative forms than men may signify a change from below in Australia and New Zealand. Overall, the relative strength of gender is weaker than both age and region when we compare the corresponding ranges in all four languages (see Table 7.6). 


\begin{tabular}{|c|c|c|c|c|}
\hline $\begin{array}{r}\text { Input probability } \\
\text { non-citation form percentage }\end{array}$ & $\begin{array}{l}\text { HKSL }(\mathrm{N}=645) \\
0.178 \\
22\end{array}$ & $\begin{array}{l}\text { ASL }(\mathrm{N}=2594) \\
0.518 \\
53\end{array}$ & $\begin{array}{l}\text { Auslan }(\mathrm{N}=2667) \\
0.427 \\
45\end{array}$ & $\begin{array}{l}\text { NZSL }(\mathrm{N}=2096) \\
0.412 \\
43\end{array}$ \\
\hline \multicolumn{5}{|l|}{ Age } \\
\hline Younger & $0.619(18-34)$ & $0.602(15-25)$ & $0.565(<51)$ & \\
\hline Middle & $0.548(35-49)$ & $0.517(26-54)$ & & n.s. \\
\hline Older & $0.337(\geqslant 50)$ & $0.416(\geqslant 55)$ & $0.411(\geqslant 51)$ & \\
\hline Range & 28 & 19 & 15 & \\
\hline \multicolumn{5}{|l|}{ Gender } \\
\hline Female & & 0.451 & 0.536 & 0.536 \\
\hline Male & n.s. & 0.544 & 0.460 & 0.460 \\
\hline Range & & 9 & 8 & 8 \\
\hline \multicolumn{5}{|l|}{ Region } \\
\hline Larger/urban & & 0.529 (Other sites) & 0.554 (Sydney, & 0.604 (South) \\
\hline & $\mathrm{N} / \mathrm{A}$ & 0.461 (Washington) & Melbourne) & 0.544 (North) \\
\hline Smaller/rural & & 0.334 (Virginia) & $\begin{array}{l}0.456 \text { (Adelaide, } \\
\text { Brisbane, Perth) }\end{array}$ & 0.417 (Central) \\
\hline Range & & 20 & 10 & 19 \\
\hline \multicolumn{5}{|l|}{ Language Background } \\
\hline Hearing parents & & 0.519 & & 0.532 (acquired $\geqslant 7$ ) \\
\hline & n.s. & & n.s. & 0.491 (acquired $<7$ ) \\
\hline Deaf parents & & 0.444 & & 0.630 \\
\hline Range & & 8 & & 14 \\
\hline \multicolumn{5}{|l|}{ Ethnicity } \\
\hline Pākehā & & \multirow{5}{*}{$\begin{array}{l}\text { Ethnicity } \times \text { Social Class } \\
0.555(\mathrm{C}, \mathrm{MC}, \& \mathrm{WC}) \\
0.445(\mathrm{AA}, \mathrm{MC}) \\
0.314(\mathrm{AA}, \mathrm{WC})\end{array}$} & & 0.513 \\
\hline Māori & $\mathrm{N} / \mathrm{A}$ & & $\mathrm{N} / \mathrm{A}$ & 0.423 \\
\hline Range & & & & 9 \\
\hline Social Class & & & & \\
\hline $\begin{array}{l}\text { Middle } \\
\text { Working }\end{array}$ & $\mathrm{N} / \mathrm{A}$ & & n.s. & $\mathrm{N} / \mathrm{A}$ \\
\hline Range & & 24 & & \\
\hline \multicolumn{5}{|l|}{ Work Environment } \\
\hline Non-deaf related & 0.603 & & & \\
\hline Deaf related & 0.397 & $\mathrm{~N} / \mathrm{A}$ & $\mathrm{N} / \mathrm{A}$ & $\mathrm{N} / \mathrm{A}$ \\
\hline Range & 21 & & & \\
\hline
\end{tabular}

Table 7.6: Social factors considered in HKSL, ASL, Auslan, and NZSL (application value: non-citation form, NB: studies differ in social factors examined)

In both ASL and NZSL, language background reaches statistical significance but with different results. On the one hand, the native ASL signers disfavour the non-citation form. On the other hand, the native NZSL signers strongly favour it. However, language background is found to be not significant in both Auslan and HKSL. Lucas et al. (2001) explained the conservative behaviour of native signers as being protective or loyal towards a more 'proper' form of ASL. Seeing a different pattern in Auslan and 
NZSL, Schembri et al. (2009) suggested "it may be that lowering of signs in these communities has not yet become the focus of any social awareness or stigmatization" (p. 223). For the case of HKSL, the sample size of native signers may be too small to show any impact. Since the social factors of ethnicity and social class are not included in the current study for the ethnographic reasons stated earlier, they are not discussed here. The factor of work environment will be discussed later for it is only coded and analyzed in the present study of HKSL.

\subsubsection{Language change in progress?}

In the studies of ASL and Auslan, researchers proposed that the lowering of signs, those articulated at the forehead area in particular, is an example of language change in progress. Their argument was based on the apparent time hypothesis and evidence from real-time research data (Bailey et al., 1991; Bailey, 2004). By applying the apparent-time construct, synchronic data can reflect diachronic linguistic development and language change in progress (Labov, 1994). That is to say, research showing different variant choice between old and young language users can be a plausible indicator of language change over time. Employing a real time approach, earlier research in ASL and BSL (BSL is the same family as Auslan and NZSL) reported that centralization of signs or moving towards neutral space happened with respect to location change in signs (as described in Section 2.3.2). More recently, similar research comparing Taiwan Sign Language (TSL) publications of different times to explore variation and historical change was conducted in Taiwan (Chen \& Tai, 2009). The findings of this TSL study suggested that signs made in the face area tended 
to be lowered in TSL (see Section 2.3 .2 on page 17). Notwithstanding the fact that TSL and HKSL belong to different language families, both languages share a similar cultural background and both are in constant contact with the same written Chinese, though different spoken Chinese languages. There is also contact between these two sign languages. TSL may be a good reference for considering change in HKSL.

As presented earlier, age is the strongest and most significant social constraint in the location variable of HKSL in the Rbrul analysis. Age was selected as significant in three of the four classification trees. Both analytical tools show that younger signers produced significantly more noncitation forms than older signers. This age variation may be an indication of language change in progress in HKSL, parallel to the case of ASL and Auslan. Figure 7.6 illustrates the percentage of non-citation forms by age and grammatical class using the dataset containing deaf school name signs, which are in the category of proper noun. The majority of tokens are verbs and adjectives, which occupy over $70 \%$ of the data $(55 \%$ and $16 \%$ respectively). In these two major categories, the younger signers used the highest percentage of non-citation forms, whereas the older signers produced the lowest. 


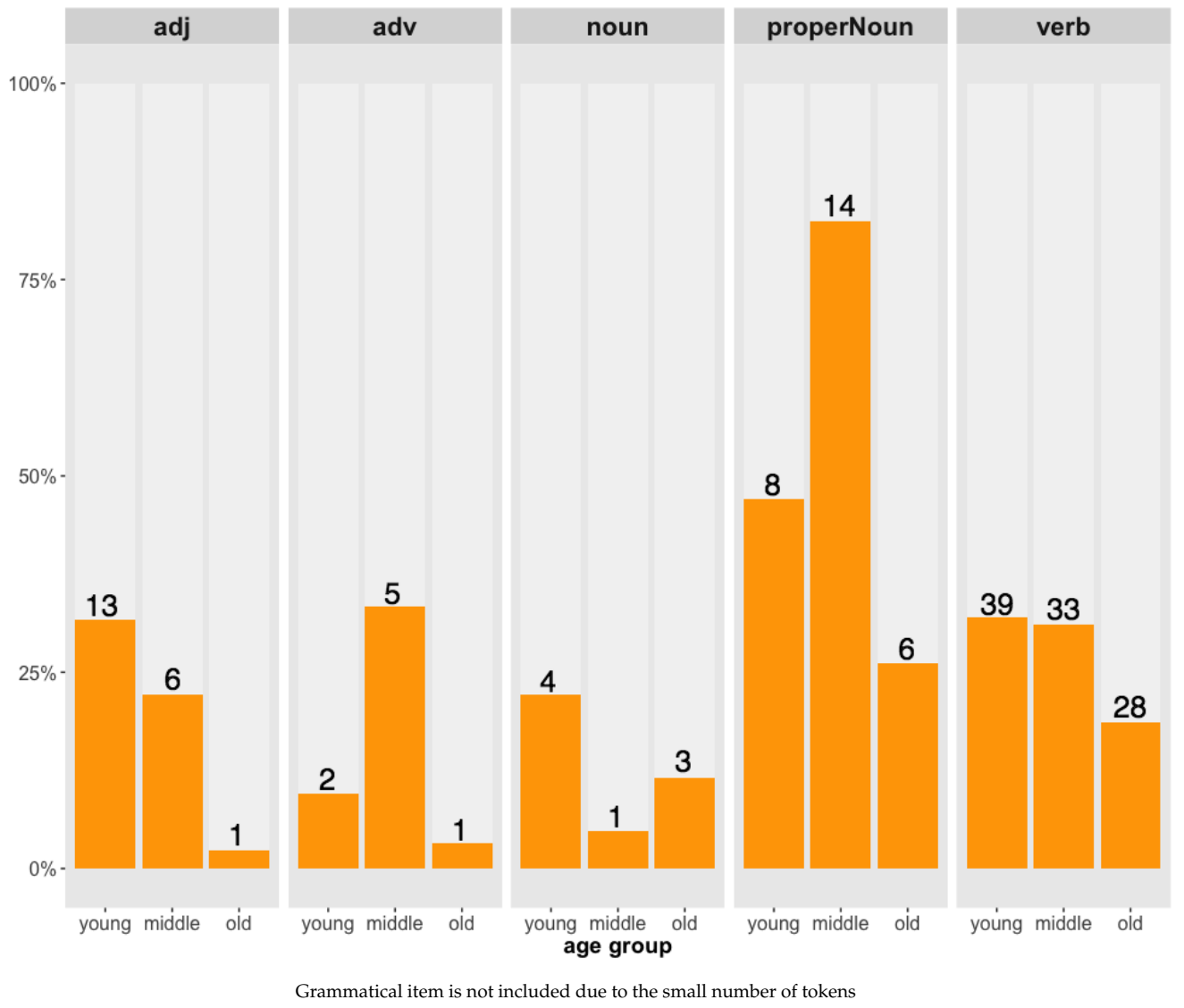

Figure 7.6: Percentage of non-citation forms by age and grammatical class

Another piece of evidence is from the distribution of tokens by age and school, which is displayed in Figure 7.7. Only HSD+ and LSD have data in all three age groups and both illustrate that older signers made more citation forms than younger and middle-aged signers. This confirms the general impression of change across generations shown in Figure 7.6. Signers from LSD produced the highest percentage of non-citation forms in all three age groups. Younger signers seem to produce fewer non-citation forms then middle-aged signers, especially those from HSD+. However, this may be due to the fact that all younger signers from HSD+ work in a deaf related environment. On the contrary, the younger signers from 
CMS+ produce slightly more non-citation forms than their middle-aged counterparts. Since OMS+ only has older signers, no further information can be derived from the data. The overall number and percentage of noncitation forms produced by younger and middle-aged signers seem very alike. However, when we look at the breakdown by work environment, this similar pattern between younger and middle-aged signers can be explained. As shown in Figure 7.8, no middle-aged signers work in a deaf related setting. When we compare the percentage of non-citation forms used by signers who have a non-deaf related job, the discrepancy between younger and middle-aged group is more apparent.

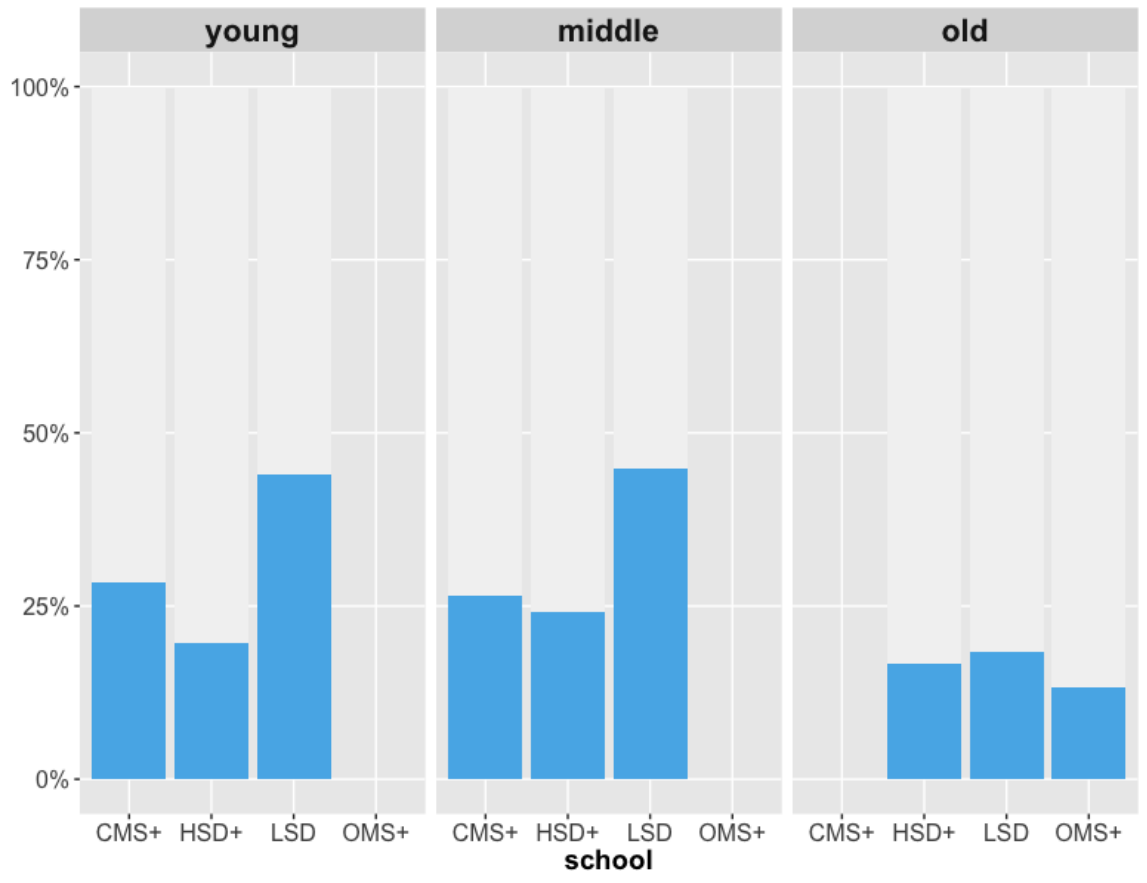

Figure 7.7: Percentage of non-citation forms by age and school 


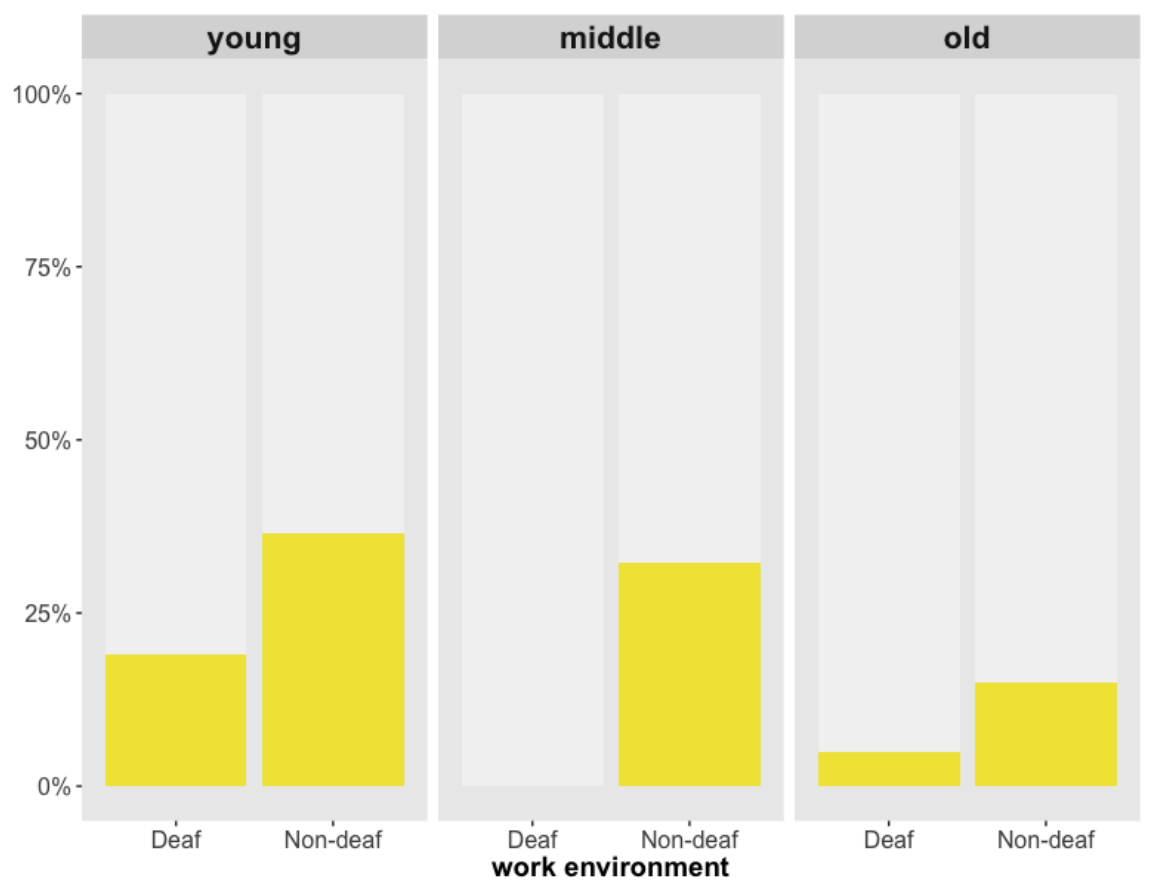

Figure 7.8: Percentage of non-citation forms by age and work environment

In view of the influence of work environment as suggested in the Rbrul analysis, I removed tokens made by signers working in deaf related organizations and further analyzed the distribution by age and gender. The result is shown in Figure 7.9. Without the effect of work environment, the difference between younger and middle-aged signers is more obvious, with younger signers producing more non-citation forms than middleaged signers for both female and male. Supporting the results of the classification trees reported earlier, men produced more non-citation forms than women in both younger and middle-aged groups. Younger men used noncitation forms the most, with nearly half of their tokens lowered, followed by middle-aged men, while younger and middle-aged women used far less. As in the case of ASL, women are more conservative in the use of this innovative lowered form. Inasmuch as LSD signers produced the most 
innovative forms, it may evidence that this change is led by young male LSD signers.

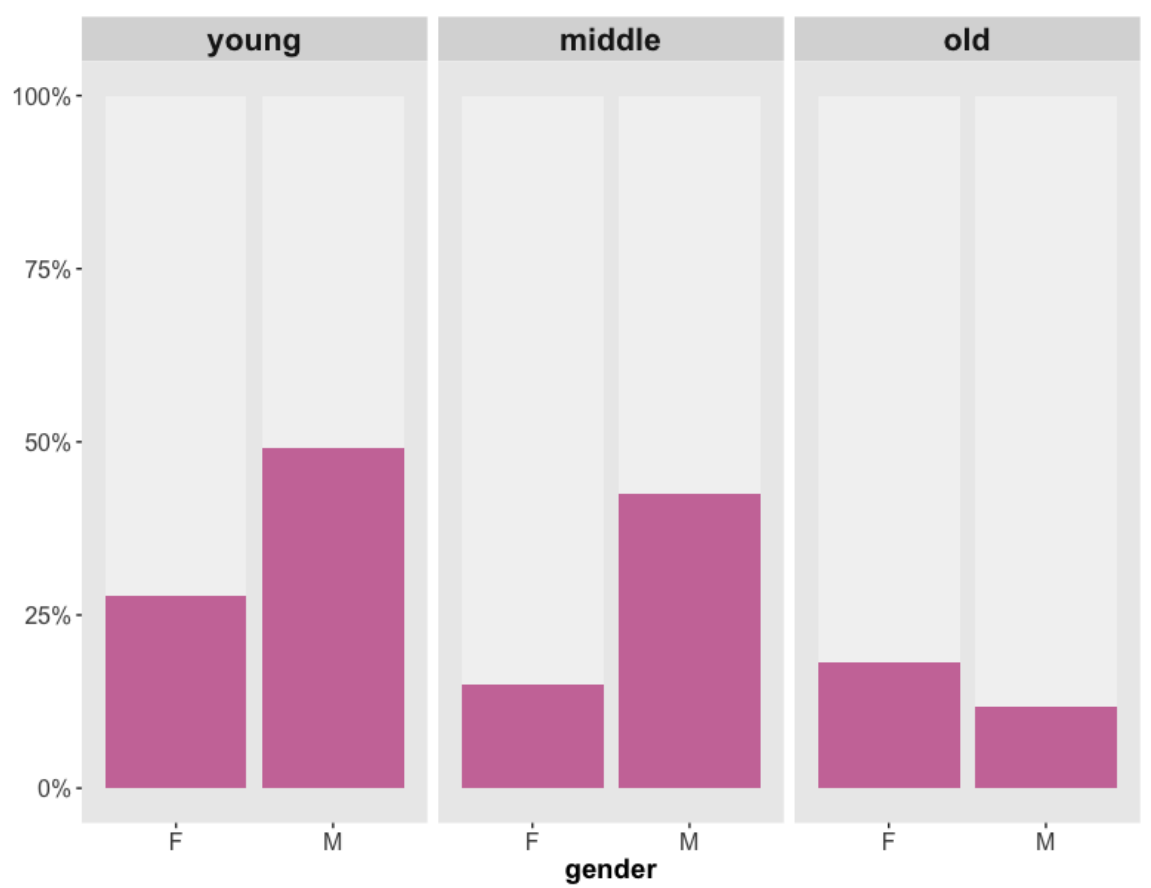

Figure 7.9: Percentage of non-citation forms by age and gender (excluding signers working in deaf-related environment)

\subsubsection{The deaf school name signs}

Deaf school is not only the cradle of sign language and deaf identity as previously stated, it also reveals a deaf individual's background when growing up. When two deaf persons meet for the first time, one of the most common opening questions is "YOU IN-THE-PAST STUDY WHAT? (Which school did you go to?)" and the answer would simply be one of the deaf school name signs. This simple answer, however, means much more than just a school name, but a bunch of connotations linked with the name. Knowing which school(s) a deaf friend had attended is regarded as es- 
sential information in the Hong Kong deaf community. Apart from being the information exchanged when two deaf individuals first met, deaf school name signs also appear frequently in deaf conversations. For example, in the conversation data, two deaf men were talking about sports. One recently joined a running group. He talked about the group before he joined and said, "RUN ALTOGETHER FORTY-SOMETHING ... HSD MANY CMS VSD MANY ONE LSD ONE ... (There are over 40 members in the running group ... many are from HSD, many from CMS and also VSD, just one from LSD, just one ...)" In another conversation between two women, one talked about playing game in a gathering. She depicted, "IX HSD GROUP IX LSD GROUP IX HARD-OF-HEARING IX CMS FOUR-IN-TOTAL ... (There are four different groups: HSD, LSD, hard-of-hearing, and CMS)." This kind of data illustrates how important a person's schooling is seen as within the Hong Kong deaf community, it transcends discussions of education and background and is used as a way of classifying individuals in many contexts.

Among the several deaf school name signs, three of them happened to be made at the forehead/temple area. They are HSD, LSD, and HIUCHONG and they rank 10,12, and 17 respectively among 75 target signs (see Table 4.3 on page 110). This shows that these name signs are not infrequent in the conversation data. Unlike the studies of Auslan and NZSL, lexical frequency fails to reach significance and high frequency verbs do not significantly favour non-citation forms in the HKSL data. Rather deaf school name signs demonstrate a similar pattern, and there is significantly more lowering in these three signs as compared to the other target signs (see Figure 7.10). This may reflect the 'salient' characteristic of this group of signs and thus explain the greater phonological reduction. 


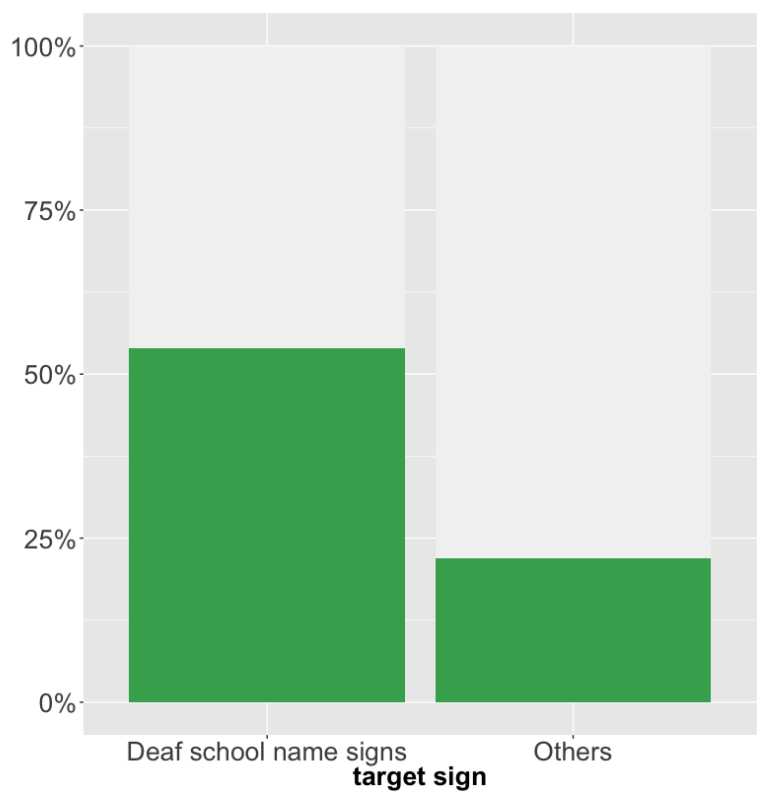

Figure 7.10: Percentage of non-citation forms by target sign

Kerswill and Williams (2002) proposed a three-component model of salience, suggesting that the presence of a dynamic linguistic phenomenon such as language change and variation, language-internal explanations like phonetic distance, and extra-linguistic factors are essential components for the notion of salience to operate. They state that the last component is indeed the cause of salience, as "sociolinguistic factors, particularly social psychological ones, come to the fore in influencing the adoption or non-adoption of linguistic forms" (p. 83). In the case of deaf school name signs, the unique role of deaf school has set its name signs apart from other proper nouns like place names and country names. The forms of these name signs are clearly associated with specific meanings, hence they would hardly be missed or misinterpreted in discourse, even if their forms are reduced. Thus, the notion of 'salience' may be used to explain why the three deaf school name signs are more frequently lowered than other types 
of forehead-located signs.

Furthermore, it is believed that formational changes in signs are motivated by two basic principles: ease of articulation and ease of perception (Frishberg, 1975). Lowering of deaf school name signs at the forehead location apparently fits the first principle while not violating the second. The evidence on the distribution of lowered variants (discussed in the previous section) suggests that this language change probably started in these salient signs when used by the signers from these schools, particularly those from LSD.

\subsubsection{Deaf related work environment}

In the Rbrul results reported earlier, whether a signer works in a deaf related environment is a significant and strong constraint on the variant choice of the location variable. Signers who have a deaf related job disfavour non-citation forms and those who are not working in such organizations favour them. In the sample, seven out of the forty participants work in a deaf related setting. These settings include CSLDS, the only deaf school LSD, one of the deaf organizations, and a deaf restaurant. As mentioned earlier, CSLDS is the only institute that conducts sign language research and provides related training in Hong Kong. Most of the deaf staff work as research assistants for their HKSL research projects or teaching assistants for the Sign Bilingualism and Co-enrolment in Deaf Education 
Programme. ${ }^{6}$ Their duties may include teaching HKSL, being an informant in HKSL research, and acting as a model for HKSL teaching materials. Naturally, deaf colleagues are considered as role models of HKSL for hearing colleagues. As role models, they may feel 'pressure' to produce the 'proper' form of signs in all situations. This 'proper' form, very often, is the citation form as it appears in the dictionary and is taught in sign language classes. A similar situation holds for the signers who work in LSD and deaf organization, one works as a sign language demonstrator for the school's Sign Assisted Instruction Programme ${ }^{7}$, and the other is responsible for sign language teaching in addition to her other duties.

Finally, the deaf restaurant is a social enterprise with an aim to support the disadvantaged by hiring deaf and hearing-impaired people. Actually, it is quite different from the three deaf related organizations above. By nature, it is more like a non-deaf related company, which employs a number of deaf workers, such as a packaging factory. But the main difference between these non-deaf related companies and the deaf restaurant is the role of deaf staff and their language at the workplace. In the former, deaf workers are hired to perform their duties and whether the hearing co-workers learn to sign or not is a matter of personal interest. However, since having deaf staff is a way of promoting the restaurant, and the hearing coworkers are encouraged to learn some signs, HKSL becomes part of the institution's marketing strategy and the deaf workers are considered to be

\footnotetext{
${ }^{6}$ Information about the Sign Bilingualism and Co-enrolment in Deaf Education Programme can be found at their website: www.cuhk.edu.hk/cslds/jcslco/.

${ }^{7}$ Information about the Sign Assisted Instruction Programme can be found at their website: www.sign-aip.net/tc/home/index.php.
} 
language models for teaching and promoting the use of sign. Therefore, perceived 'pressure' to use citation forms may be felt by these signers.

This variation pattern in relation to work environment may reflect that the innovative non-citation form is perceived as an 'improper' or 'lazy' form of signing. Hence, signers who feel responsible to 'model' HKSL in their work role prefer the traditional citation form.

\subsection{Chapter summary}

In this chapter, I have shown that the location variable in the class of signs produced at or near the forehead area is a classic sociolinguistic variable in signed languages. The occurrence of non-citation forms in HKSL is far less common than in the other languages studied. It seems that the older the language is, the higher the proportion of non-citation forms used. In accordance with the previous studies in ASL, Auslan, and NZSL, this sociolinguistic variable co-varies in HKSL with both linguistic and social factors in a systematic way. In terms of linguistic constraints, while similarities and dissimilarities are observed across the four languages, related languages (Auslan and NZSL) share more commonality with each other than unrelated ones. Moreover, while the current study affirms the important role of grammatical class, the combined effect of grammatical class and lexical frequency found in the Auslan and NZSL studies is not replicated in the HKSL data. Like ASL and Auslan, the result in the current study has suggested that the lowering of this class of signs in HKSL is also a language change in progress led by younger signers. The findings further indicate that this change has originated in the deaf school name signs due 
to their salience and that signers from these schools have led the change, particularly the men from LSD. Yet, this change may be in an early stage as reflected in the overall low percentage of the innovative form and reflecting the young age of the language. Lastly, work environment was found to be a significant and strong constraint on variant choice. Signers who work in a deaf related setting disfavour the non-citation form suggesting that this innovative lowered form is probably viewed as having lower prestige in the Hong Kong deaf community. 



\section{Chapter 8}

\section{Conclusions and limitations}

In this thesis, I have investigated lexical and phonological variation in HKSL by employing a quantitative method. The results of the current study confirm that variation in HKSL is pervasive, but it is not in a state of 'chaos.' Rather, the language has its "orderly heterogeneity" and its variation is correlated with social and linguistic factors in a systematic way, as has been observed in other spoken and signed languages.

The analysis of the lexical items has shown that variant choices are correlated with signer's age, the school(s) one has attended, and to a lesser extent, whether one is a man or a woman. Further investigation of the use of compounds, 'citation forms,' and the handedness of number signs has demonstrated the complex relationship between the social factors. Five social factors: age, school, gender, education, and work environment, interact in various ways to condition the different variant choices.

The findings affirm the lay belief that older signers sign differently 
than younger signers. The fact that older signers use fewer variants than middle-aged and younger signers is linked directly to the language policy in deaf schools. Most of the older signers went to signing schools, while the middle-aged and younger signers were educated orally. The substantial range of lexical variants used among the middle and younger cohorts is definitely an outcome of oral-only deaf education during 1970s to 2000s. There is evidence that some 'old' forms are fading away and some 'modern' forms are spreading. Nonetheless, older signers also use 'modern' forms, indicating a lifelong lexical adaptation. Most of the changes observed in the lexical items conform to those found in other signed languages.

School differentiation is not only manifested in the lexicon, but also in the way one uses compounds, one-handed or two-handed number signs, and phonological features. Nevertheless, those school related variants have reached signers of other schools. Similar to the regional variation observed in other sign languages, it is more of a degree rather than a categorical variant preference. The findings seem to suggest that there are school 'dialects,' comparable to the regional dialects in national sign languages (indeed these regional dialects originate in the regional schools as explained in Chapter 2). Yet, these school 'dialects' are constantly in contact, making them distinct from regional dialects in some way. This kind of 'dialects in contact' situation in sign language deserves further investigation as it may shed some light on understanding language contact and contact-induced change. Unlike spoken dialects in contact, contact in sign languages relies heavily on face-to-face interaction - media influence on HKSL is still minimal if any. Nonetheless, the influence of technology (i.e., the increasing video interflow through various electronic devices) has yet 
to be explored. More importantly, it is foreseeable that only two schoollects will remain in the future. These will be that of LSD, as it is now the only deaf school in Hong Kong, and that of HSD, as most of the deaf workers in the Centre for Sign Linguistics and Deaf Studies (CSLDS) are from HSD and many of them teach at their co-enrolment programme.

Although the effect of gender is not as prominent as those of age and school, it recurs in various variant choices. Females tend to use more citation forms than males, both in the case of lexical items and in the location variable, which is in accordance with the ASL study and some Cantonese studies (Pan, 1981; Yeung, 1980). Like the findings in British Sign Language (BSL), men favour two-handed number signs more than women (Stamp et al., 2015). In addition, gender is also found to be a significant factor in the use of compounds, the variant choice of HEARING, and several lexical items where handshape is concerned. This last observation suggests future research directions on the effects of gender on the use of phonological features like handshape. We already know that gender effects may be seen in dichotomous variables such as prestige vs non-prestige forms, in the use of handedness, and in signing space (Wulf, 1998). But we have not known much about how it may correlate with the formational parameters in signed languages.

One of the most prominent findings of the current study is the role of grammatical constraints in conditioning the two phonological variables examined. Although different methods are employed to investigate the movement pattern in DEAF/ HEARING and the location variable in the class of signs articulated at or near the forehead, both results have demonstrated the strong effect of grammatical constraint. This parallels the re- 
sults of previous studies (Lucas et al., 2001). The immediate phonological environment also plays a role in the location variable and probably in HEARING, but it is less important than the grammatical class. It may be worth to explore whether grammatical class would also be influential to lexical variation in the future.

The results have suggested there is a change in both phonological variables. I have proposed that two different types of change are happening in DEAF. One is linguistically driven (originating from compounds) and the other is socially driven (motivated by redefining deaf identity). Yet, this is just a preliminary investigation. Such change deserves further research for its importance to the deaf community. For location drop, the change appears to have started from the deaf school name signs due to their salience, and young signers from these schools have led the change, particularly the men from LSD. This change may be in an early stage as compared with the change detected in ASL and Auslan. Clearly, research on this classic variable in more signed languages would help to verify the conclusions reached in the current analysis.

With regard to the limitations of this study, the lexical items selected are those expected to vary based on my observation. The results reported have inevitably been shaped by the choice of the items. Moreover, the lexical data is obtained through the decontextualized elicitation task, which means the results may not inform us about the complete repertoire of signers in discourse of various contexts. As we have seen in the comparison of some data in elicitations and conversations, a signer may use different variants according to the audience, context, and situation.

As I have emphasized in Chapter 4, the issues of multicollinearity and 
collinearity exist in the datasets. Even though measures have been taken to alleviate the situation, the problem may still affect the results to a certain extent. I am a hearing non-native signer and the only coder of all the data, and the coding schemes are largely built on the previous studies. Hence, there may be a concern of coding reliability and validity. Nevertheless, being both an insider and outsider of the deaf community may have granted me distinct insights in analyzing and explaining the data.

Lastly, this study is the first to investigate sociolinguistic variation in HKSL using a quantitative method. It has followed the methodology adopted in the ASL, Auslan, and NZSL studies, which allowed the comparison between the four languages and thus offered a better understanding of variation and change in signed languages to a certain extent. Despite the limitations of the study, it has provided a foundation for future research and has practical implications on sign language teaching, interpreter training, and dictionary making. More importantly, this study has taken a 'snapshot' of a rapidly changing language and has documented some signs which may disappear in the near future. 



\section{References}

Adam, R. (2012). Language contact and borrowing. In R. Pfau, M. Steinbach, \& B. Woll (Eds.), Sign language: An international handbook (pp. 841862). Berlin, Germany: De Gruyter Mouton.

Ann, J. (1998). Contact between a sign language and a written language: Character signs in Taiwan Sign Language. In C. Lucas (Ed.), Pinky extension and eye gaze: Language use in deaf communities (pp. 59-99). Washington, DC: Gallaudet University Press.

Antoons, I. \& Boonen, D. (2004). Hard and hard and hard: The same sign? On the evolution of signs in Flemish Sign Language. In M. Van Herreweghe \& M. Vermeerbergen (Eds.), To the lexicon and beyond: Sociolinguistics in European deaf communities (pp. 74-88). Washington, DC: Gallaudet University Press.

Armstrong, N. (2001). Social and stylistic variation in spoken French: A comparative approach. Amsterdam, Netherlands: John Benjamins.

Ash, S. (2013). Social class. In J. K. Chambers \& N. Schilling (Eds.), The handbook of language variation and change (2nd ed, pp. 350-367). Oxford, England: John Wiley \& Sons. 
Austin, P. K. (2010). Current issues in language documentation. In P. K. Austin (Ed.), Language documentation and description (Vol. 7, pp. 1233). London, England: SOAS.

Baguley, T. (2012). Serious stats: A guide to advanced statistics for the behavioral sciences. New York, NY: Palgrave Macmillan.

Bailey, G. (2004). Real and apparent time. In J. K. Chambers, P. Trudgill, \& N. Schilling-Estes (Eds.), The handbook of language variation and change (pp. 312-332). Oxford, England: Blackwell.

Bailey, G., Wikle, T., Tillery, J., \& Sand, L. (1991). The apparent time construct. Language Variation and Change, 3(3), 241-264. doi:10.1017 / S0954394500000569

Baker, R. \& Knight, P. (1998). 'Total communication' - Current policy and practice. In S. Gregory, P. Knight, W. McCracken, S. Powers, \& L. Watson (Eds.), Issues in deaf education (pp. 77-87). London, England: Fulton.

Battison, R. (1974). Phonological deletion in American Sign Language. Sign Language Studies, 5(1), 1-19.

Battison, R. (1978). Lexical borrowing in American Sign Language. Silver Spring, MD: Linstok Press.

Battison, R., Markowicz, H., \& Woodward, J. (1975). A good rule of thumb: Variable phonology in American Sign Language. In R. W. Fasold \& R. Shuy (Eds.), Analyzing variation in language (pp. 291-302). Washington, DC: Georgetown University Press.

Bauer, R. S. \& Benedict, P. K. (1997). Modern Cantonese phonology. Trends in linguistics. Berlin, Germany: Mouton de Gruyter.

Bayard, D. (1989). 'me say that? no way!': The social correlates of American lexical diffusion in New Zealand English. Te Reo, 32, 17-60. 
Bayley, R. \& Lucas, C. (2011). Sign languages. In R. Mesthrie (Ed.), The Cambridge handbook of sociolinguistics (pp. 83-102). Cambridge, England: Cambridge University Press.

Beal, J. C. (2010). An introduction to regional Englishes: Dialect variation in England. Edinburgh, Scotland: Edinburgh University Press.

Bell, A. (1984). Language style as audience design. Language in Society, 13(2), 145-204. doi:10.2307/4167516

Boberg, C. (2004). Real and apparent time in language change: Late adoption of changes in Montreal English. American Speech, 79(3), 250-269.

Boberg, C. (2010). The English language in Canada: Status, history and comparative analysis. Cambridge, England: Cambridge University Press.

Bourgerie, D. S. (1990). A quantitative study of sociolinguistic variation in Cantonese (Doctoral dissertation, The Ohio State University).

Brentari, D. (1998). A prosodic model of sign language phonology. Language, speech, and communication. Cambridge, MA: MIT Press.

Bybee, J. (2002). Word frequency and context of use in the lexical diffusion of phonetically conditioned sound change. Language variation and change, 14(3), 261-290.

Caritas Magdalene School. (2000). Caritas Magdalene School 25th anniversary of the founding jubilee commemorative magazine. Hong Kong: Author.

Census and Statistics Department. (2014). Social data collected via the general household survey: Special topics report - Report no.62. Hong Kong: Author.

Centre for Sign Linguistics and Deaf Studies. (2015). Centre for Sign Linguistics and Deaf Studies: Publications. Retrieved from http:/ / www. cslds.org/v3/publications.php?page $=2$ 
Chambers, J. K. (1992). Dialect acquisition. Language, 68(4), 673-705. doi:10. $2307 / 416850$

Chambers, J. K. (2009). Sociolinguistic theory: Linguistic variation and its social significance (Rev. ed.). Language in society. Chichester, England: Blackwell.

Chambers, J. K. \& Trudgill, P. (1980). Dialectology. Cambridge textbooks in linguistics. Cambridge, England: Cambridge University Press.

Chambers, J. K., Trudgill, P., \& Schilling-Estes, N. (Eds.). (2004). The handbook of language variation and change. Oxford, England: Blackwell.

Chambers, J. K. (2000). Region and language variation. English World-Wide, 21(2), 169-199.

Chen, Y. \& Tai, J. H. Y. (2009). Lexical variation and change in Taiwan Sign Language. In J. H. Y. Tai \& J. Tsay (Eds.), Taiwan Sign Language and beyond (pp. 131-148). Chia-Yi, Taiwan: The Taiwan Institute for the Humanities, National Chung Cheng University.

Cheshire, J. (2004). Sex and gender in variationist research. In J. K. Chambers, P. Trudgill, \& N. Schilling-Estes (Eds.), The handbook of language variation and change (pp. 423-443). Oxford, England: Blackwell.

Crasborn, O. \& Sloetjes, H. (2008). Enhanced ELAN functionality for sign language corpora. In Proceedings of the 3rd Workshop on the Representation and Processing of Sign Languages: Construction and Exploitation of Sign Language Corpora (pp. 39-43).

Crasborn, O. \& Sloetjes, H. (2014). Improving the exploitation of linguistic annotations in ELAN. In N. Calzolari, K. Choukri, T. Declerck, H. Loftsson, B. Maegaard, J. Mariani, ...S. Piperidis (Eds.), Proceedings of LREC 2014: 9th International Conference on Language Resources and Evaluation (pp. 3604-3608). 
DeSantis, S. (1977). Elbow to hand shift in French and American Sign Languages. Paper presented at the annual NWAVE conference, Georgetown University, Washington, DC.

Deuchar, M. (1981). Variation in British Sign Language. In B. Woll, J. Kyle, \& M. Deuchar (Eds.), Perspectives on British Sign Language and deafness (pp. 109-119). Guildford, England: Billing.

Deuchar, M. (1984). British Sign Language. London, England: Routledge \& Kegan Paul.

Dinkin, A. J. (2008). The real effect of word frequency on phonetic variation. University of Pennsylvania Working Papers in Linguistics, 14(1), Article 8 .

Eckert, P. (1997). Age as a sociolinguistic variable. In F. Coulmas (Ed.), The handbook of sociolinguistics (pp. 151-167). Oxford, England: Blackwell.

Eddington, D. (2010). A comparison of two tools for analyzing linguistic data: Logistic regression and decision trees. Italian Journal of Linguistics, 22(2), 265-286.

Eichmann, H. \& Rosenstock, R. (2014). Regional variation in German Sign Language: The role of schools (re-)visited. Sign Language Studies, 14(2), 175-202. doi:10.1353/sls.2014.0001

Farfán, J. A. F. \& Ramallo, F. F. (2010). New perspectives on endangered languages: Bridging gaps between sociolinguistics, documentation and language revitalization. Amsterdam, Netherlands: John Benjamins.

Fenlon, J., Schembri, A., Rentelis, R., \& Cormier, K. (2013). Variation in handshape and orientation in British Sign Language: The case of the ' 1 ' hand configuration. Language \& Communication, 33(1), 69-91. doi:10.1016/j.langcom.2012.09.001 
Fischer, S. \& Gong, Q. (2010). Variation in east Asian sign language structures. In D. Brentari (Ed.), Sign languages (pp. 499-518). Cambridge, England: Cambridge University Press.

Frishberg, N. (1975). Arbitrariness and iconicity: Historical change in American Sign Language. Language, 51(3), 696-719.

Frisina, R. (1963). On the educational aspects of deafness in Hong Kong. Report submitted to the Education Department of the Hong Kong Government.

Geraci, C., Battaglia, K., Cardinaletti, A., Cecchetto, C., Donati, C., Giudice, S., \& Mereghetti, E. (2011). The LIS corpus project: A discussion of sociolinguistic variation in the lexicon. Sign Language Studies, 11(4), 528-574. doi:10.1353/sls.2011.0011

Glynn, D. (2014). The many uses of run: Corpus methods and socio-cognitive semantics. In D. Glynn \& J. A. Robinson (Eds.), Corpus methods for semantics: Quantitative studies in polysemy and synonymy (pp. 117-144). Amsterdam, Netherlands: John Benjamins.

Goodstadt, R. Y. C. (1972). Speaking with signs: A sign language manual for Hongkong's deaf. Hong Kong: Government Printer.

Grenoble, L. (2010). Language vitality and revitalization in the Arctic. In J. Farfan \& F. F. Ramallo (Eds.), New perspectives on endangered languages: Bridging gaps between sociolinguistics, documentation and language revitalization (pp. 65-91). Amsterdam, Netherlands: John Benjamins.

Grenoble, L. (2013). Unanswered questions in language documentation and revitalization. In E. Mihas, B. Perley, G. Rei-Doval, \& K. Wheatley (Eds.), Responses to language endangerment: New directions in lan- 
guage documentation and language revitalization (pp. 43-57). Amsterdam, Netherlands: John Benjamins.

Guy, G. R. \& Bobery, C. (1997). Inherent variability and the obligatory contour principle. Language Variation and Change, 9, 149-164.

Hair, J. F., Black, W. C., Babin, B. J., Anderson, R. E., \& Tatham, R. L. (2006). Multivariate data analysis. Upper Saddle River, NJ: Pearson Prentice Hall.

Hale, K., Krauss, M., Watahomigie, L. J., Yamamoto, A. Y., Craig, C., Jeanne, L. M., \& England, N. C. (1992). Endangered languages. Language, 68(1), 1-42. doi:10.2307/416368

Halliday, M. A. K. (1975). Some aspects of sociolinguistics. In E. Jacobsen (Ed.), Interactions between linguistics and mathematics education: Final report of the symposium sponsored by UNESCO, CEDO and ICMI, Nairobi, Kenya, September 1-11, 1974 (UNESCO Report No. ED-74/CONF.808) (pp. 64-73). Paris, France: UNESCO.

Himmelmann, N. (1998). Documentary and descriptive linguistics. Linguistics, 36, 161-195.

Himmelmann, N. P. (2008). Reproduction and preservation of linguistic knowledge: Linguistics' response to language endangerment. Annual Review of Anthropology, 37(1), 337-350. doi:10.1146 / annurev. anthro.37.081407.085226

Hockett, C. F. (1950). Age-grading and linguistic continuity. Language, 26(4), 449-457. doi:10.2307/410396

Holmes, J. (2001). An introduction to sociolinguistics (2nd ed). New York, NY: Longman.

Holmes, J. \& Stubbe, M. (2003). Power and politeness in the workplace: A sociolinguistic analysis of talk at work. London, England: Pearson. 
Hong Kong Association of the Deaf. (2004). Hong Kong Sign Language 1. Hong Kong: Author.

Hong Kong School for the Deaf. (1995). Hong Kong School for the Deaf, anniversary magazine 1935-1995. Hong Kong: Author.

Hong Kong Society for the Deaf. (1985). Sign language training handbook. Hong Kong: Author.

Hong Kong Society for the Deaf. (1987). The origin and development of Hong Kong Sign Language. Hong Kong: Author.

Hong Kong Society for the Deaf. (2005). Hong Kong Sign Language (elementary). Hong Kong: Author.

Hong Kong Society for the Deaf. (2006). Hong Kong Sign Language (intermediate). Hong Kong: Author.

Hoopes, R. (1998). A preliminary examiniation of pinky extension: Suggestions regarding its occurrence, constraints, and function. In C. Lucas (Ed.), Pinky extension and eye gaze: Language use in deaf communities (pp. 3-17). Washington, DC: Gallaudet University Press.

Hoyer, K. (2007). Albanian Sign Language: Language contact, international sign, and gesture. In D. Quinto-Pozos (Ed.), Sign languages in contact (pp. 195-234). Washington, DC: Gallaudet University Press.

Hudson, R. A. (1996). Sociolinguistics (2nd ed). Cambridge textbooks in linguistics. Cambridge, England: Cambridge University Press.

Janzen, T. (2012). Lexicalization and grammaticalization. In R. Pfau, M. Steinbach, \& B. Woll (Eds.), Sign language: An international handbook (pp. 816-841). Berlin, Germany: De Gruyter Mouton.

Johnson, D. E. (2009). Getting off the GoldVarb standard: Introducing Rbrul for mixed-effects variable rule analysis. Language and Linguistics Compass, 3(1), 359-383. 
Johnson, E. (1993). The relationship between lexical variation and lexical change. Language Variation and Change, 5(3), 285-303. doi:10.1017 / S0954394500001514

Johnston, T. (2002). The representation of English using Auslan: Implications for deaf bilingualism and English literacy. Australian Journal of Education of the Deaf, 8, 23-37.

Johnston, T. (2003). BSL, Auslan and NZSL: Three signed languages or one? In A. Baker, B. van den Bogaerde, \& O. Crasborn (Eds.), Crosslinguistic perspectives in sign language research: Selected papers from TISLR 2000 (pp. 47-69). Hamburg, Germany: Signum.

Johnston, T. (2010). From archive to corpus: Transcription and annotation in the creation of signed language corpora. International Journal of Corpus Linguistics, 15(1), 106-131.

Johnston, T. A. \& Schembri, A. (2007). Australian Sign Language (Auslan): An introduction to sign language linguistics. Cambridge, England: Cambridge University Press.

Kerswill, P. \& Williams, A. (2002). "Salience" as an explanatory factor in language. In M. C. Jones \& E. Esch (Eds.), Language change: The interplay of internal, external, and extra-linguistic factors (pp. 81-110). New York, NY: Mouton de Gruyter.

Knowles, G. (1978). The nature of phonological variables in Scouse. In P. Trudgill (Ed.), Sociolinguistic patterns in British English (pp. 80-90). London, England: E. Arnold.

Kouega, J. P. (2009). Campus English: Lexical variations in Cameroon. International Journal of the Sociology of Language, 2009(199), 89-101. doi:10. 1515/IJSL.2009.036 
Kügler, F., Féry, C., \& Vijver, R. (2009). Variation and gradience in phonetics and phonology. Berlin, Germany: Mouton de Gruyter.

Labov, W. (1966). The social stratification of English in New York city. Washington, DC: Center for Applied Linguistics.

Labov, W. (1972). Sociolinguistic patterns. Philadelphia: University of Pennsylvania Press.

Labov, W. (1994). Principles of linguistic change: Vol. 1, Internal factors. Oxford, England: Blackwell.

Labov, W., Ash, S., \& Boberg, C. (2006). The atlas of north American English: Phonetics, phonology, and sound change: A multimedia reference tool. New York, NY: Mouton de Gruyter.

Langacker, R. W. (1988). A usage-based model. In B. Rudzka-Ostyn (Ed.), Topics in cognitive linguistics (pp. 127-161). Current Issues in Linguistic Theory, 50. Amsterdam, Netherlands: John Benjamins.

Langacker, R. W. (1999). A dynamic usage-based model. In S. Kemmer \& M. Barlow (Eds.), Usage-based models of language (pp. 1-63). Stanford, CA: CSLI.

Lee, H., Tsay, J., \& Myers, J. (2001). Handshape articulation in Taiwan Sign Language and Signed Chinese. Paper presented at the Conference on Sign Linguistics, Deaf Education and Deaf Culture in Asia, Chinese University of Hong Kong.

Leeson, L. \& Grehan, C. (2004). To the lexicon and beyond: The effect of gender on variation in Irish Sign Language. In M. Van Herreweghe \& M. Vermeerbergen (Eds.), To the lexicon and beyond: Sociolinguistics in European deaf communities (pp. 39-73). Washington, DC: Gallaudet University Press. 
Leeson, L. \& Saeed, J. I. (2012). Irish Sign Language: A cognitive linguistic account. Edinburgh, Scotland: Edinburgh University Press.

LeMaster, B. (2002). What difference does difference make? Negotiating gender and generation in Irish Sign Language. In S. Benor, M. Rose, D. Sharma, J. Sweetland, \& Q. Zhang (Eds.), Gendered practices in language (pp. 309-338). Stanford, CA: CSLI.

Levshina, N. (2015). Conditional inference trees and random forests. In How to do linguistics with $R$ : Data exploration and statistical analysis (pp. 291-300). Amsterdam, Netherlands: John Benjamins.

Liddell, S. K. (1984). THINK and BELIEVE: Sequentiality in American Sign Language. Language, 60(2), 372-399. doi:10.2307/413645

Liddell, S. K. (1993). Holds and positions: Comparing two models of segmentation in ASL. In G. Coulter (Ed.), Current issues in ASL phonology (pp. 189-211). San Diego, CA: Academic Press.

Liddell, S. K. \& Johnson, R. E. (1986). American Sign Language compound formation processes, lexicalization, and phonological remnants. Natural Language and Linguistic Theory, 4(4), 445-513. doi:10.1007/BF00134470

Liddell, S. K. \& Johnson, R. E. (1989). American Sign Language: The phonological base. Sign Language Studies, 195-277.

Lodge, R. A. (2004). A sociolinguistic history of Parisian French. New York, NY: Cambridge University Press.

Lucas, C. (1995). Sociolinguistic variation in ASL: The case of DEAF. In C. Lucas (Ed.), Sociolinguistics in deaf communities (pp. 3-25). Washington, DC: Gallaudet University Press.

Lucas, C. \& Bayley, R. (2010). Variation in American Sign Language. In D. Brentari (Ed.), Sign languages (pp. 451-475). Cambridge, England: Cambridge University Press. 
Lucas, C., Bayley, R., Rose, M., \& Wulf, A. (2002). Location variation in American Sign Language. Sign Language Studies, 2(4), 407-440. doi:10. 1353/sls.2002.0020

Lucas, C., Bayley, R., \& Valli, C. (2001). Sociolinguistic variation in American Sign Language. Washington, DC: Gallaudet University Press.

Lucas, C. \& Valli, C. (1992). Language contact in the American deaf community. San Diego, CA: Academic Press.

Lutheran School for the Deaf. (2009). Lutheran School for the Deaf: About the school. Retrieved from http:/ / www.lsd.edu.hk/about_school. php

Lytle, R. R., Johnson, K. E., \& Hui, Y. J. (2005). Deaf education in China: History, current issues, and emerging deaf voices. American Annals of the Deaf, 150(5), 457-469. doi:10.1353/aad.2006.0009

McKee, D. \& Kennedy, G. (1999). A list of 1,000 frequently used signs in New Zealand Sign Language. In G. Kennedy (Ed.), New Zealand Sign Language: Distribution, origins, reference (Occasional Publication 2) (pp. 17-25). Wellington, New Zealand: Deaf Studies Research Unit, Victoria University.

McKee, D. \& Kennedy, G. D. (2006). The distribution of signs in New Zealand Sign Language. Sign Language Studies, 6(4), 372-390.

McKee, D., McKee, R., \& Major, G. (2011). Numeral variation in New Zealand Sign Language. Sign Language Studies, 12(1), 72-97.

McKee, R. \& McKee, D. (2011). Old signs, new signs, whose signs? Sociolinguistic variation in the NZSL lexicon. Sign Language Studies, 11(4), 485-527, 660. 
McKee, R., Schembri, A., McKee, D., \& Johnston, T. (2011). Variable “subject" presence in Australian Sign Language and New Zealand Sign Language. Language Variation and Change, 23(3), 375-398.

Menard, S. (2002). Applied logistic regression analysis. Thousand Oaks, CA: SAGE.

Metzger, M. (1993). Pronoun variation in formal and informal discourse. In E. Winston (Ed.), Communication forum 1993 (pp. 132-143). Washington, DC: Gallaudet University, Department of Linguistics and Interpreting.

Meyerhoff, M. (2011). Introducing sociolinguistics (2nd ed). New York, NY: Routledge.

Meyerhoff, M. (2015). Turning variation on its head: Analysing subject prefixes in Nkep (Vanuatu) for language documentation. Asia-Pacific Language Variation, 1(1), 78-108.

Milroy, J. (1982). Probing under the tip of the iceberg: Phonological 'normalization' and the shape of speech communities. In S. Romaine (Ed.), Sociolinguistic variation in speech communities (pp. 35-47). London, England: E. Arnold.

Milroy, J. \& Milroy, L. (1978). Belfast: Change and variation in an urban vernacular. In P. Trudgill (Ed.), Sociolinguistic patterns in British English (pp. 19-36). London, England: E. Arnold.

Milroy, L. (1987a). Language and social networks (2nd ed). Language in society. Oxford, England: Blackwell.

Milroy, L. (1987b). Observing and analysing natural language: A critical account of sociolinguistic method. Language in society. Oxford, England: Blackwell. 
Milroy, L. \& Gordon, M. (2003). Sociolinguistics: Method and interpretation. Language in society. Malden, MA: Blackwell.

Mitchell, R. E. \& Karchmer, M. A. (2004). Chasing the mythical ten percent: Parental hearing status of deaf and hard of hearing students in the United States. Sign Language Studies, 4(2), 138-163. doi:10.1353/sls. 2004.0005

Moineddin, R., Matheson, F. I., \& Glazier, R. H. (2007). A simulation study of sample size for multilevel logistic regression models. BMC Medical Research Methodology, 7(1), 34. doi:10.1186/1471-2288-7-34

Nadasdi, T., Mougeon, R., \& Rehner, K. (2008). Factors driving lexical variation in L2 French: A variationist study of automobile, auto, voiture, char and machine. Journal of French Language Studies, 18(3), 365-381. doi:10.1017/S0959269508003505

Nagy, N. (2011). Lexical change and language contact: Faetar in Italy and Canada. Journal of Sociolinguistics, 15(3), 366-382. doi:10.1111/j.14679841.2011.00488.x

National Cued Speech Association. (n.d.). Cued speech definition. Retrieved from http://www.cuedspeech.org/cued-speech-definition.php

Nevalainen, T. \& Raumolin-Brunberg, H. (2003). Historical sociolinguistics: Language change in Tudor and Stuart England. London, England: Pearson Education.

Nonaka, A., Mesh, K., \& Sagara, K. (2015). Signed names in Japanese Sign Language: Linguistic and cultural analyses. Sign Language Studies, 16(1), 57-85. doi:10.1353/sls.2015.0025

O'Brien, R. M. (2007). A caution regarding rules of thumb for variance inflation factors. Quality and Quantity, 41(5), 673-690. 
Pan, P. G. (1981). Prestige forms and phonological variation in Hong Kong Cantonese speech (MA Thesis, The University of Hong Kong).

Paolillo, J. C. (2013). Individual effects in variation analysis: Model, software, and research design. Language Variation and Change, 25(1), 89118. doi:10.1017/S0954394512000270

Penn, C. \& Reagan, T. (1994). The properties of South African Sign Language: Lexical diversity \& syntactic unity. Sign Language Studies, 85(1), 319-327. doi:10.1353/sls.1994.0011

Perlmutter, D. M. (1991). Prosodic vs segmental structure: A moraic theory of American Sign Language syllable structure. Unpublished manuscript, University of California, San Diego.

Phillips, B. S. (1984). Word frequency and the actuation of sound change. Language, 60(2), 320-342.

Phua, M. (2003). The language skills of Singaporean deaf children using total communication (MA Thesis, National University of Singapore).

Plaza-Pust, C. (2012). Deaf education and bilingualism. In R. Pfau, M. Steinbach, \& B. Woll (Eds.), Sign language: An international handbook (pp. 949979). Berlin, Germany: De Gruyter Mouton.

Pope, J., Meyerhoff, M., \& Ladd, D. R. (2007). Forty years of language change on Martha's Vineyard. Language, 83(3), 615-627.

Quinn, G. (2010). Schoolization: An account of the origins of regional variation in British Sign Language. Sign Language Studies, 10(4), 476-501. doi:10.1353/sls.0.0056

Radutzky, E. (1989). La Lingua dei Segni Italiana: Historical change in the sign language of deaf people in Italy (Doctoral dissertation, New York University). 
Radutzky, E. (2009). Il cambiamento fonologico storico della lingua dei segni italiana. In C. Bertone \& A. Cardinaletti (Eds.), Alcuni capitoli della grammatica della LIS: Atti dell'Incontro di studio "La grammatica della Lingua dei segni italiana" (pp. 17-42). Venice, Italy: Cafoscarina.

Rehabilitation Division, Labour and Welfare Bureau. (2008). Hong Kong rehabilitation programme plan 2005-2007. Hong Kong: Author.

Robinson, J. (2012). Lexical variation in the BBC voices recordings. English Today, 28(4), 23-37. doi:10.1017/S0266078412000363

Sandler, W. (1986). The spreading hand autosegment of American Sign Language. Sign Language Studies, 50(1), 1-28.

Sandler, W. (1989). Phonological representation of the sign: Linearity and nonlinearity in American Sign Language. Berlin, Germany: Walter de Gruyter.

Sandler, W. \& Lillo-Martin, D. C. (2006). Sign language and linguistic universals. Cambridge, England: Cambridge University Press.

Schembri, A. (2010). Documenting sign languages. In P. K. Austin (Ed.), Language documentation and description (Vol. 7, pp. 105-143). London, England: SOAS.

Schembri, A. \& Johnston, T. (2004). Sociolinguistic variation in Auslan (Australian Sign Language): A research project in progress. Deaf Worlds, 20(1), 78-90.

Schembri, A. \& Johnston, T. (2012). Sociolinguistic aspects of variation and change. In R. Pfau, M. Steinbach, \& B. Woll (Eds.), Sign language: An international handbook (pp. 788-816). Berlin, Germany: De Gruyter Mouton.

Schembri, A., McKee, D., McKee, R., Pivac, S., Johnston, T., \& Goswell, D. (2009). Phonological variation and change in Australian and New 
Zealand Sign Languages: The location variable. Language Variation and Change, 21(2), 193-231.

Schermer, G. M. (2003). From variant to standard: An overview of the standardization process of the lexicon of Sign Language of the Netherlands over two decades. Sign Language Studies, 3(4), 469-486. doi:10. 1353/sls.2003.0017

Schilling-Estes, N. (2007). Sociolinguistic fieldwork. In R. Bayley \& C. Lucas (Eds.), Sociolinguistic variation: Theories, methods, and applications (pp. 165-189). Cambridge, England: Cambridge University Press.

Senghas, R. J., Senghas, A., \& Pyers, J. E. (2005). The emergence of Nicaraguan Sign Language: Questions of development, acquisition, and evolution. In S. T. Parker, J. Langer, \& C. Milbrath (Eds.), Biology and knowledge revisited: From neurogenesis to psychogenesis (pp. 287-306). New York, NY: Lawrence Erlbaum Associates.

Shroyer, E. H. \& Shroyer, S. P. (1984). Signs across America: A look at regional differences in American Sign Language. Washington, DC: Gallaudet College Press.

Skinner, R. A. (2007). What counts? A typological and descriptive analysis of British Sign Language number variations (MA Thesis, Birkbeck College, University of London).

Smith, W. H. (2005). Taiwan Sign Language research: An historical overview. Language and Linguistics, 6(2), 187-215.

Stamp, R., Schembri, A., Fenlon, J., \& Rentelis, R. (2015). Sociolinguistic variation and change in British Sign Language number signs: Evidence of leveling? Sign Language Studies, 15(2), 151-181. 
Stamp, R., Schembri, A., Fenlon, J., Rentelis, R., Woll, B., \& Cormier, K. (2014). Lexical variation and change in British Sign Language. PLoS ONE, 9(4), e94053. doi:10.1371/journal.pone.0094053

Stokoe, W. C., Casterline, D., \& Cronberg, C. G. (1965). A dictionary of American Sign Language on linguistic principles. Silver Spring, MD: Linstok Press.

Sutton-Spence, R. \& Woll, B. (1999). The linguistics of British Sign Language: An introduction. Cambridge, England: Cambridge University Press.

Suvannus, S. (1987). Thailand. In J. V. Van Cleve (Ed.), Gallaudet encyclopedia of deafness and deaf people (pp. 282-284). Washington, DC: Gallaudet University Press.

Svartvik, J. \& Leech, G. N. (2006). English: One tongue, many voices. New York, NY: Palgrave Macmillan.

Sze, F., Chu, K., Lo, C., \& Lo, L. (2013). Lexical variations and diachronic change in Hong Kong Sign Language. Poster session presented at TISLR 11, University College London, England.

Sze, F., Lo, C., Lo, L., \& Chu, K. (2011). Early deaf education in Hong Kong and its relation with the origin of Hong Kong Sign Language. Education Journal, 39(1), 139-156.

Sze, F., Lo, C., Lo, L., \& Chu, K. (2013). Historical development of Hong Kong Sign Language. Sign Language Studies, 13(2), 155-185.

Tagliamonte, S. (2006). Analysing sociolinguistic variation. Key topics in sociolinguistics. Cambridge, England: Cambridge University Press.

Tagliamonte, S. (2012). Variationist sociolinguistics: Change, observation, interpretation. Language in society. Malden, MA: Wiley-Blackwell.

Tang, G. (Ed.). (2007). Hong Kong Sign Language: A trilingual dictionary with linguistic descriptions. Hong Kong: Chinese University Press. 
Tang, G., Yiu, C. K.-M., \& Lam, S. (2015). Awareness of Hong Kong Sign Language and manually coded Chinese by deaf students learning in a sign billingual and co-enrollment setting: A Hong Kong case study. In H. Knoors \& M. Marschark (Eds.), Educating deaf learners: Creating a global evidence base (pp. 117-148). New York, NY: Oxford University Press.

Trudgill, P. (1986). Dialects in contact. Language in society. Oxford, England: Basil Blackwell.

Tyrone, M. E. \& Mauk, C. E. (2010). Sign lowering and phonetic reduction in American Sign Language. Journal of Phonetics, 38(2), 317-328. doi:10.1016/j.wocn.2010.02.003

Upton, C. \& Widdowson, J. D. A. (2006). An atlas of English dialects. New York, NY: Routledge.

Van Herreweghe, M. \& Vermeerbergen, M. (2004). Flemish Sign Language: Some risks of codification. In M. Van Herreweghe \& M. Vermeerbergen (Eds.), To the lexicon and beyond: Sociolinguistics in European deaf communities (pp. 111-135). Washington, DC: Gallaudet University Press.

Van Hoek, K., Bellugi, U., \& Fok, A. (1986). Sign language universals: Evidence from Chinese Sign Language. Working paper. Salk Institute, University of California, San Diego \& The University of Hong Kong.

Vanhecke, E. \& Weerdt, K. D. (2004). Regional variation in Flemish Sign Language. In M. Van Herreweghe \& M. Vermeerbergen (Eds.), To the lexicon and beyond: Sociolinguistics in European deaf communities (pp. 27-38). Washington, DC: Gallaudet University Press.

Vicars, W. (2015). Learn American Sign Language (ASL). Retrieved from http://lifeprint.com/ 
Wagenmakers, E.-J. (2007). A practical solution to the pervasive problems of p values. Psychonomic Bulletin and Review, 14(5), 779-804.

Wardhaugh, R. (2001). An introduction to sociolinguistics (4th ed). Blackwell textbooks in linguistics. Malden, MA: Blackwell.

Weinreich, U., Labov, W., \& Herzog, M. (1968). Empirical foundations for a theory of language change. In W. P. Lehmann \& Y. Malkiel (Eds.), Directions for historical linguistics: A symposium (pp. 95-188). Austin: University of Texas Press.

Wilbur, R. B. (1987). American Sign Language: Linguistic and applied dimensions (2nd ed). Boston, MA: Little, Brown.

Winzer, M. A. (1993). Education, urbanization, and the deaf community: A case study of Toronto, 1870-1900. In J. V. Van Cleve (Ed.), Deaf history unveiled: Interpretations from the new scholarship (pp. 127-145). Washington, DC: Gallaudet University Press.

Wolfram, W. \& Schilling-Estes, N. (2006). American English: Dialects and variation (2nd ed). Language in society. Malden, MA: Blackwell.

Woll, B. (1987). Historical and comparative aspects of British Sign Language. In J. G. Kyle (Ed.), Sign and school: Using signs in deaf children's development (pp. 12-34). Clevedon, England: Multilingual Matters.

Woll, B., Sutton-Spence, R., \& Elton, F. (2001). Multilingualism: The global approach to sign languages. In C. Lucas (Ed.), The sociolinguistics of sign languages (pp. 8-32). Cambridge, England: Cambridge University Press.

Woodbury, T. (2011). Language documentation. In P. Austin \& J. Sallabank (Eds.), The Cambridge handbook of endangered languages (pp. 159-186). Cambridge, England: Cambridge University Press. 
Woodward, J. (1993). Intuitive judgments of Hong Kong signers about the relationship of sign language varieties in Hong Kong and Shanghai. CUHK Papers in Linguistics, 4, 88-96.

Woodward, J. (1996). Modern Standard Thai Sign Language, influence from ASL, and its relationship to original Thai sign varieties. Sign Language Studies, 92(1), 227-252. doi:10.1353/sls.1996.0012

Woodward, J. \& De Santis, S. (1977). Two to one it happens: Dynamic phonology in two sign languages. Sign Language Studies, 17, 329-346.

Woodward, J., Erting, C., \& Oliver, S. (1976). Facing and hand(1)ing variation in American Sign Language phonology. Sign Language Studies, 10(1), 43-51.

Wulf, A. (1998). Gender related variation in ASL signing space. Guided research paper, Gallaudet University. Washington, DC.

Yau, S. C. (1977). The Chinese signs: Lexicon of the standard sign language for the deaf in China. Hong Kong: Chiu Ming.

Yeung, S. W. H. (1980). Some aspects of phonological variations in the Cantonese spoken in Hong Kong (MA Thesis, The University of Hong Kong).

Yi, Y. M. (2008). Report on the investigation into the phonology of Shanghai variety of Chinese Sign Language (Doctoral dissertation, Fudan University, China).

Young, R. \& Bayley, R. (1996). VARBRUL analysis for second language acquisition research. In R. Bayley \& D. R. Preston (Eds.), Second language acquisition and linguistic variation (pp. 253-306). Amsterdam, Netherlands: John Benjamins.

Yu, B. (2014). Historical documentation of Hong Kong Sign Language used in Canossa School for the Deaf/Caritas Magdalene School (MA Thesis, The Chinese University of Hong Kong). 



\section{Appendices}

\section{A Methodological tools}

A1. Confidentiality agreement for Research Assistants

A2. Participant information sheet

A3. Participant consent form

A4. Participant demographic profile

A5. Interview questions 


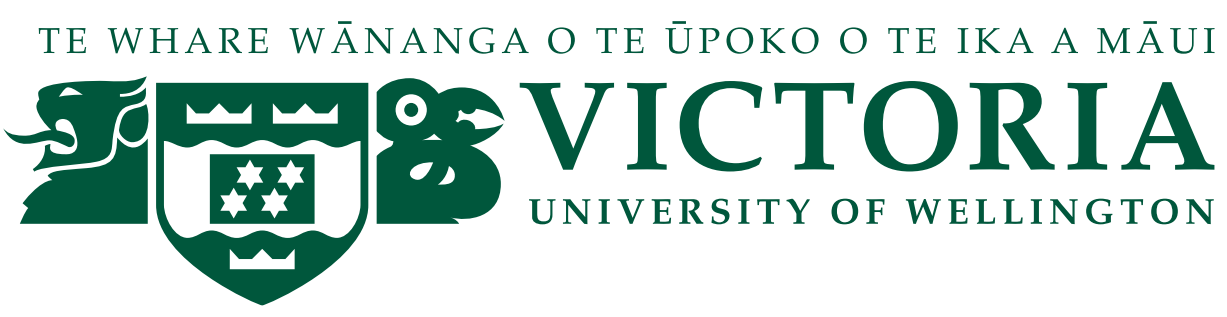

\author{
Deaf Studies Research Unit \\ School of Linguistics and Applied Language Studies
}

\title{
Confidentiality Agreement for Research Assistants
}

Project title: Sociolinguistic Variation of Hong Kong Sign Language (HKSL)

I understand that this confidentiality agreement applies to all information I have access to while working with video recordings or any other data collected as part of this project.

I agree to keep all information confidential and to securely store and transmit any digital or paper files in my possession, in accordance with

- DSRU research procedures;

- the approval(s) given by a VUW Human Ethics Committee

Any content arising from the data that affects me personally will be discussed with the leader of the research project.

Name.

Signature

Witness

Signature.

Date 
TE WHARE WĀNANGA O TE ÜPOKO O TE IKA A MĀUI

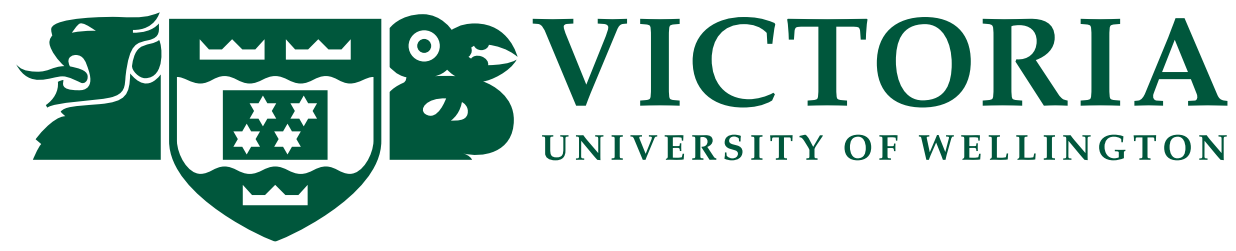

\author{
語言學及應用語言學系 \\ 壟人研究部
}

研究助理保密協議

研究項目 : 香港手語的詞彙變異

本人明白這份保密協議適用於所有有關這項研究計劃的資料, 包括我所接觸到的錄 影記錄或任何其他收集得來的資料。

本人同意履行保密義務, 並按照壟人研究部的研究程序, 以及威靈頓維多利亞大學 操守委員會的批准, 安全地存儲和傳送我所接觸到的任何數據或文件檔案。

如有任何資料內容, 對我個人造成影響，我將會與研究負責人商討。

姓名：

簽署 :

見證人：

簽署：

日期 : 


\section{Research project: Lexical Variation in Hong Kong Sign Language (HKSL)}

\section{INFORMATION SHEET FOR PARTICIPANTS}

I am a PhD student in Applied Linguistics at Victoria University of Wellington. The project I am undertaking is investigating lexical variation in Hong Kong Sign Language (HKSL). This research project has received approval from the Victoria University Human Ethics Committee.

\section{PROCEDURES}

You will be invited to do 3 tasks if you agree to participate in this project. It will take about 2 hours to finish all 3 tasks.

1. Free conversation: you will have a conversation with 1 or 2 friend(s) of yours for about 45-60 minutes. Only 2 or 3 of you will be involved in the conversation and it will be video recorded.

2. Sign the word: I will show you a set of 120 flashcards with a picture and a word. You will show me how you sign each concept. This will take about 10-15 minutes. Your signing will be video recorded.

3. Interview: you will be interviewed individually. You will be asked about your everyday experiences, for example, which deaf school(s) did you go to? How often do you use HKSL? The interview will last for about 30 minutes and it will be video recorded.

\section{COMPENSATION FOR PARTICIPATION}

After completing all 3 tasks, you will be given a HK\$200 supermarket voucher as a compensation for your time.

\section{CONFIDENTIALITY}

The information you give me will be private and confidential. Your name will not be on the video files. I will keep the files in a safe place. I will use them to write a thesis for the University. The thesis will be submitted for marking to the School of Linguistics and Applied Language Studies and deposited in the University Library. I will keep your recording only if you allow me to use it for other linguistic research purposes. Only linguistic researchers will be allowed to view the recording.

\section{PARTICIPATION AND WITHDRAWAL}

Your participation is voluntary. This means that you can choose to stop at any time while doing the tasks without negative consequences. If you want to withdraw from the project, you may do so before $1^{\text {st }}$ March, 2014, and any data you have provided will be destroyed.

\section{QUESTIONS AND CONCERNS}

If you have any further questions or would like to receive further information about the project, please contact me, or my supervisors, David McKee and Rachel McKee, at the School of Linguistics and Applied Language Studies at Victoria University, PO Box 600, Wellington, New Zealand.

Rebecca Siu: +852 9733-9155 or rebecca.siu@vuw.ac.nz

David McKee: +852 6146-9161 or david.mckee@vuw.ac.nz

Rachel McKee: +64 44635640 (fax) or rachel.mckee@vuw.ac.nz 


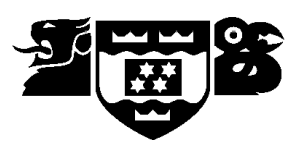

\section{研究項目：香港手語的詞彙變異}

\section{研究參與者須知}

本人是新西蘭威靈頓維多利亞大學應用語言學的博士研究生, 現正進行一項有關香港手語的 詞彙變異的研究。此項研究已獲得維多利亞大學的操守委員會批准。

\section{程序}

如果你同意參加這項研究, 你會被邀請做以下三件事項, 共需約2個小時。

1. 談話：你將與1-2位你的朋友自由交談約45-60分鐘，除你們2-3人以外, 不會有其他人參與 你們的談話, 而你們的談話將會被錄影。

2. 手語詞彙 : 我將會給你看一組共 120 張圖咭 / 圖片連字咭, 請你告訴我每張圖咭的手語。 所需時間約為10-15分鐘, 你的手語將會被錄影。

3. 面談 : 你將單獨與我面談, 我會請你分享你的個人經驗, 如 : 你以前讀哪間壟校 ? 你有多 常用香港手語 ? 面談為時約 30 分鐘, 你的面談內容將會被錄影。

\section{參與報酬}

完成全部錄影後，你將獲得價值港幣200元的超市禮券作為參與研究的報酬。

\section{保密}

你提供的所有資料, 包括你的手語錄影和個人資料表, 均會絕對保密。你的手語錄影檔案將 不會有你的名字, 這些錄影檔案都會保存在一個安全的地方。我將會分析這些資料, 並寫成 我的研究論文。這論文將會提交到語言學及應用語言研究學系評分, 並將存放在大學圖書館 內。如果你允許我使用你的手語錄影用作其他語言學研究之用, 我將會保存你的手語錄影檔 案, 只有語言學研究人員才可以查看那些檔案。

\section{參與和退出}

你的參與是完全自願性的, 你可以選擇在任何時候退出, 即使在錄影中途, 也可隨時要求停 止, 並且不會造成任何負面影響。如果你想退出是項研究, 你可以在2014年3月1日之前提出, 你所提供的資料, 將會被銷毁。

\section{查詢和意見}

如果你對是項研究有任何查詢或意見, 請與我或我的研究監督人David McKee 或 Rachel McKee 查詢, 地址是 School of Linguistics and Applied Language Studies, Victoria University of Wellington, PO Box 600, Wellington 6140, New Zealand.

$$
\text { 蕭慧欣 : +852 9733-9155 or rebecca.siu@vuw.ac.nz }
$$

David McKee: +852 6146-9161 or david.mckee@vuw.ac.nz

Rachel McKee: +64 44635640 (fax) or rachel.mckee@vuw.ac.nz 


\section{CONSENT TO PARTICIPATE IN RESEARCH}

Research project: Lexical Variation in Hong Kong Sign Language (HKSL)

\section{Name of participant:}

$>$ I have been given an information sheet about this project and I understand what it is about.

$>$ I have had an opportunity to ask questions and have them answered to my satisfaction.

$>$ I understand that I can withdraw from this project at any time before $1^{\text {st }}$ March, 2014, without giving any reasons and without any penalty of any sort. I understand that if I withdraw from the project, any data I have given will be destroyed.

$>$ I agree that the recording and transcript of my signing videos may be held by the researcher.

$>$ I understand that only linguistic researchers will be allowed to view the recording and it will only be used for linguistic research purposes.

I understand that published results will not use my name and that no opinions will be attributed to me in any way that will identify me.

$\square \quad$ I would like to receive a copy of my signing videos.

$\square \quad$ I would like to receive a summary of the final report.

Address:

Contact no.:

$\square \quad$ I allow the researcher to use my signing videos for other linguistic research purposes.

I agree to take part in this research.

Signed:

Date: 


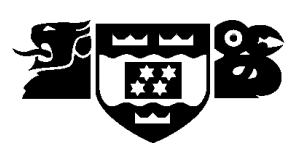

\section{參與研究同意書 \\ 研究項目 : 香港手語的詞彙變異}

參加者姓名:

> 本人已收到研究參與者須知, 並明白其內容。

$>$ 本人已對不明白的地方提出問題, 並已得到滿意的答覆。

> 本人明白我可於2014年3月1日前退出這個研究計劃, 不須作任何解釋, 且沒 有需要作出任何賠償。本人明白如果我退出計劃, 我所提供的資料, 將會被 銷毁。

$>$ 本人同意研究員保存我的錄影記錄, 以及其文字本。

$>$ 本人明白只有語言學研究人員才有權觀看我的錄影記錄, 而這些記錄亦只會 用作語言學研究的用途。

$>$ 本人明白所有我的個人資料, 將不會出現於任何文獻或公開刊物中。

口我想收到一份我的手語錄影記錄。

口我想收到一份研究報告摘要。

郵寄 / 電郵地址 :

聯絡電話：

口我同意讓研究員用我的手語錄影記錄作其他語言學研究。

我同意參與是項研究。

簽署:

日期 : 
姓名 Name：

手機 / 傳真 Phone/Fax No. :

出生日期 Date of birth： 年齡 Age :

你在香港住了 年 How long have you been living in Hong Kong?

你住在哪裡 ? Where do you live?

你在上址住了 年 How long have you been living here?

你幾多歲失去聽覺 ? At what age did you lose your hearing?

你幾多歲學手語? At what age did you learn sign?

\section{家庭背景資料 Family Background}

1. 你有其他壟 / 弱聽的家庭成員 / 親戚嗎 ? Do you have deaf/hard of hearing family members/relatives?

$\square$ 沒有 No

口有, 是誰 ? Yes, who?

2. 你有家庭成員懂手語嗎 ? Do your family members sign?

$\square$ 沒有 No

口有, 是誰? Yes, who?

3. 你現和誰同住? Who are you living with?

口配偶 / 伴侶 spouse/partner

$\square$ 父母 parents

$\square$ 兄弟姊妹 siblings

口子女 children

口其他, 請註明 others, please specify:

\section{教育背景資料 Educational Information}

4. 你的教育程度是 Educational attainment :

口幾年小學 a few year of primary education

口完成小六 finished primary 6

$\square$ 中五或以下 secondary 5 or below

$\square$ 完成中五 finished secondary 5

$\square$ 職業培訓 vocational training

口大專 / 大學, 考獲證書 college/university, certificate obtained:

$\square$ 其他, 請註明 others, please specify: 
no:

\section{5. 你在哪間學校讀小學呢 ? 哪年 ? 你有住宿舍嗎 ?}

When you were in primary school, were you at: when? Boarding?

\begin{tabular}{|c|c|c|c|}
\hline & 由 from & 至 to & 寄宿 boarding \\
\hline $\begin{array}{l}\text { 華僑竩啞學校 } \\
\text { Overseas Chinese School for the Deaf and Dumb }\end{array}$ & & & \\
\hline $\begin{array}{l}\text { 龍童會, 請註明 : } \\
\text { Clubs for Deaf Children, please specify: }\end{array}$ & & & \\
\hline $\begin{array}{l}\text { 啟音學校 } \\
\text { Kai Yum School for the Deaf }\end{array}$ & & & \\
\hline $\begin{array}{l}\text { 曉莊學校 } \\
\text { Hill Chong Special School for the Deaf }\end{array}$ & & & \\
\hline $\begin{array}{l}\text { 壟啞協會學校 } \\
\text { Hong Kong Deaf and Dumb Association School }\end{array}$ & & & \\
\hline $\begin{array}{l}\text { 真鐸啟喑學校 } \\
\text { Hong Kong School for the Deaf }\end{array}$ & & & \\
\hline $\begin{array}{l}\text { 路德會啟襲學校 } \\
\text { Lutheran School for the Deaf }\end{array}$ & & & \\
\hline $\begin{array}{l}\text { 啟聲學校 } \\
\text { Victoria School for the Deaf }\end{array}$ & & & \\
\hline $\begin{array}{l}\text { 明愛達言學校 } \\
\text { Caritas Magdalene School }\end{array}$ & & & \\
\hline $\begin{array}{l}\text { 健聽學校弱聽班，請註明： } \\
\text { Special class in hearing school, please specify: }\end{array}$ & & & \\
\hline $\begin{array}{l}\text { 其他, 請註明 : } \\
\text { Others, please specify: }\end{array}$ & & & \\
\hline
\end{tabular}

\section{6. 你在哪間學校讀中學呢? 哪年? 你有住宿舍嗎?}

When you were in secondary school, were you at: when? Boarding?

\begin{tabular}{|c|c|c|c|}
\hline & 由 from & 至 to & 寄宿 boarding \\
\hline $\begin{array}{l}\text { 華僑壟啞學校 } \\
\text { Overseas Chinese School for the Deaf and Dumb }\end{array}$ & & & \\
\hline $\begin{array}{l}\text { 曉莊學校 } \\
\text { Hill Chong Special School for the Deaf }\end{array}$ & & & \\
\hline $\begin{array}{l}\text { 真鐸啟喑學校 } \\
\text { Hong Kong School for the Deaf }\end{array}$ & & & \\
\hline $\begin{array}{l}\text { 路德會啟襲學校 } \\
\text { Lutheran School for the Deaf }\end{array}$ & & & \\
\hline $\begin{array}{l}\text { 明愛達言學校 } \\
\text { Caritas Magdalene School }\end{array}$ & & & \\
\hline $\begin{array}{l}\text { 健聽學校弱聽班，請註明 : } \\
\text { Special class in hearing school, please specify: }\end{array}$ & & & \\
\hline $\begin{array}{l}\text { 其他, 請註明 : } \\
\text { Others, please specify: }\end{array}$ & & & \\
\hline $\begin{array}{l}\text { 不適用 } \\
\text { Not applicable }\end{array}$ & & & \\
\hline
\end{tabular}




\section{面談問題 \\ INTERVIEW QUESTIONS}

\section{1. 家庭背景 Family background}

- 你出生於何地? 何年?

Where, and when, were you born?

- 家庭成員 - 壟或聽人? 你在家中排行第幾?

Family members - Deaf or hearing? Position in family?

- 如何與家人溝通?

Communication with family?

\section{2. 手語名字 Name sign}

- 你的手語名字是什麼? 怎樣得來的?

What's your name sign in HKSL - how did you get it?

\section{3. 學校經驗 School experience}

- 你入讀哪（幾）所壟校?

Which deaf school(s) did you go to?

- 在學校的社交生活如何? Social life at school?

- 課堂學習如何?

Classroom learning?

- 在學校與老師和其他同學的溝通如何?

Communication with teachers and with children at school?

- 學校有沒有壟人老師?

Deaf teachers in school?

- 你有沒有入讀過普通學校?

Did you go to hearing school(s)?

- 離開聾校後, 你有沒有再進修或接受培訓?

Any further education/training?

- 如你的家庭成員也是壟人, 他/她入讀哪間壟校?

If you have deaf family member(s), which school does he/she go to? 


\section{4. 竟人社群關係網 Deaf community networks}

- 你何時/如何開始參與壟人社群? How/when did you get involved with Deaf community?

- 你參與什麼壟人活動/團體? What Deaf activities or groups do you participate in?

- 你的朋友及伴侶是壟人? 聽人? Friends \& Partner - Deaf or hearing?

- 他們是你以前的同學? 或是在其他聾校讀書的? Are they your ex-classmates? Or from other deaf schools?

- 由你參與壟人社群以來, 你覺得聾人社群有什麼改變? Has the Deaf community changed over your lifetime?

\section{5. 香港手語 $\mathrm{HKSL}$}

- 你如何學手語? 哪裡? 何時? 誰教你? Where/when/from whom did you learn HKSL?

- 在日常生活中, 你在什麼地方會用香港手語? 有多常用? Where/how often do you use HKSL in everyday life?

- 你覺得自己的手語如何? 由初學到現在, 有改變嗎? What do you think of your HKSL? Any changes in your lifetime?

\section{6. 工作 Work}

- 你現時的工作是? 以前? Occupation? (describe current and previous)

- 你的同事是壟人? 聽人? 如何溝通? 關係如何? Workmates - Deaf/hearing? Communication? Relationships?

- 你的理想工作是什麼? 你對將來有什麼展望嗎? Ideal job - future aspirations?

\section{7. 宗教 Religion}

- 你有宗教信仰嗎? 是什麼? Do you have a religion? What?

- 你經常去教會(堂)或參與宗教活動嗎? 壟人或聽人教會(堂)? How often do you go to church/religious activities? Deaf or hearing church?

- 教會(堂)有用香港手語嗎? HKSL in church? 


\section{B Number of tokens per concept}

- Australias [72]

- Beijing [69]

- BLACK [66]

- BLUe [65]

- BROWN [69]

- COFFEe [66]

- DEAF [72]

- Eight [65]

- Eighteen [66]

- elder-brother [66]

- elder-Sister [66]

- ELEVEN [70]

- father [65]

- FIFTEEN [68]

- GOLD [65]

- GRAPe [67]

- GREEN [65]

- GREY [71]

- GUANGZHOU [70]

- HEARING [67]

- HUNDRED [67]

- HUNG-HOM [65]

- INDIA [66]

- itALy [67]

- KOREa [73]

- mother [65]
- ORANGE [66]

- ORANGE (fruit) [68]

- PINK [67]

- PURPLE [64]

- RED [65]

- Relatives [66]

- SEVEN [65]

- SEVENTEEN [67]

- silver [61]

- TAIWAN [69]

- THIRTEEN [67]

- thiRTy [65]

- THREe [64]

- tWELVE [66]

- TWENTY [65]

- tWentY-eight [65]

- TWENTY-FIVE [65]

- TWENTY-ONE [66]

- TWENTY-SEVEN [66]

- TWENTY-THREe [65]

- TWENTY-TWO [66]

- VIETNAM [70]

- WHITE [65]

- yOUNGER-BROTHER [67]

- yOUNGER-SISTER [66] 


\section{List of compound signs}

1. AUstralia

2. BEIJING

3. BROWN

4. COFFEE

5. ELDER-BROTHER

6. ELDER-SISTER

7. GOLD

8. GRAPE

9. GREY

10. GUANGZHOU

11. HUNG-HOM

12. INDIA

13. KOREA

14. ORANGE (fruit)

15. PINK

16. PURPLE

17. SILVER

18. TAIWAN

19. VIETNAM

20. YOUNGER-BROTHER

21. YOUNGER-SISTER 


\section{Sign pictures from HKSL publications}

The sign pictures illustrated here are captured from the following five HKSL publications (signs that are not found in the publications are marked as unavailable):

(a) Goodstadt, Rose Yin-Chee. (1972). Speaking with signs: a sign language manual for Hongkong's deaf. Hong Kong: Government Printer.

(b) Hong Kong Society for the Deaf. (1985). Sign Language Training Handbook. Hong Kong: Author.

(c) Hong Kong Association of the Deaf. (2004). Hong Kong Sign Language 1. Hong Kong: Author.

(d) Hong Kong Society for the Deaf. (2005). Hong Kong Sign Language (Elementary). Hong Kong: Author.

Hong Kong Society for the Deaf. (2006). Hong Kong Sign Language (Intermediate). Hong Kong: Author.

(e) Tang, Gladys. (2007). Hong Kong Sign Language: a trilingual dictionary with linguistic descriptions. Hong Kong: Chinese University Press. 


\section{COLOURS}

Figure 1: Signs for BLACK

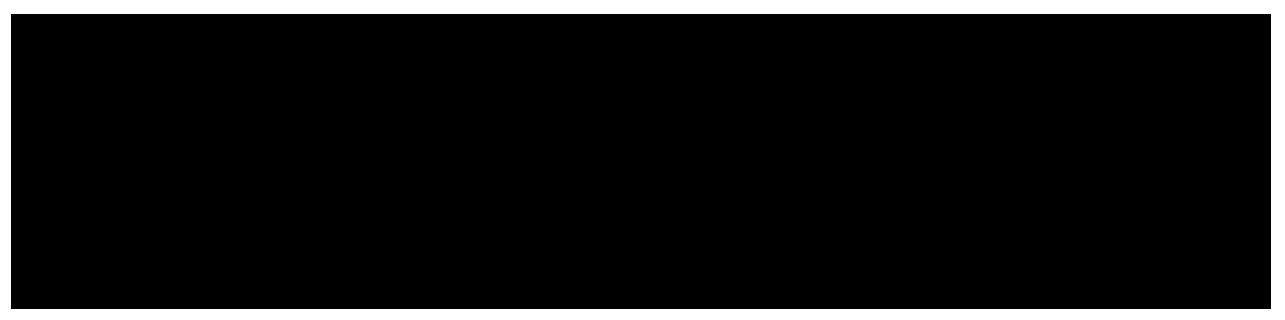

(a) 1972

(b) 1985

(c) 2004

(d) 2005

(e) 2007

Figure 2: Signs for BLUE

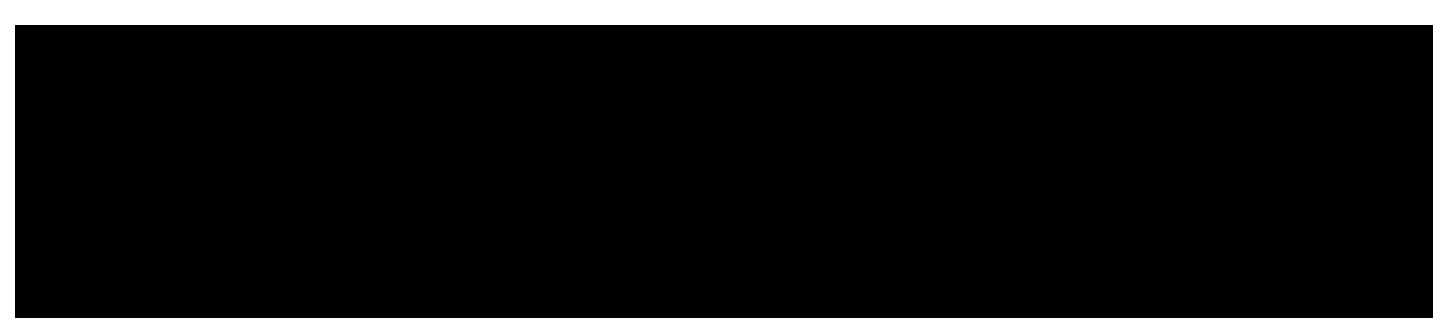
(a) 1972
(b) 1985
(c) 2004
(d) 2005
(e) 2007 
Figure 3: Signs for BROWN

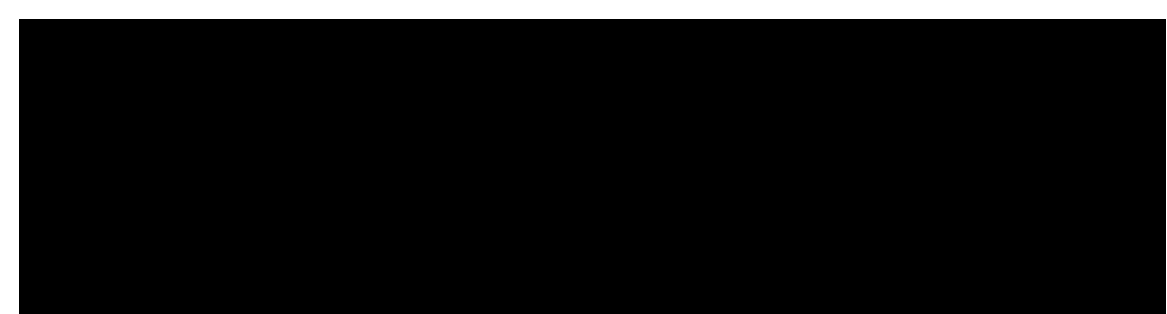
(a) 1972
(b) 1985
(c) 2004

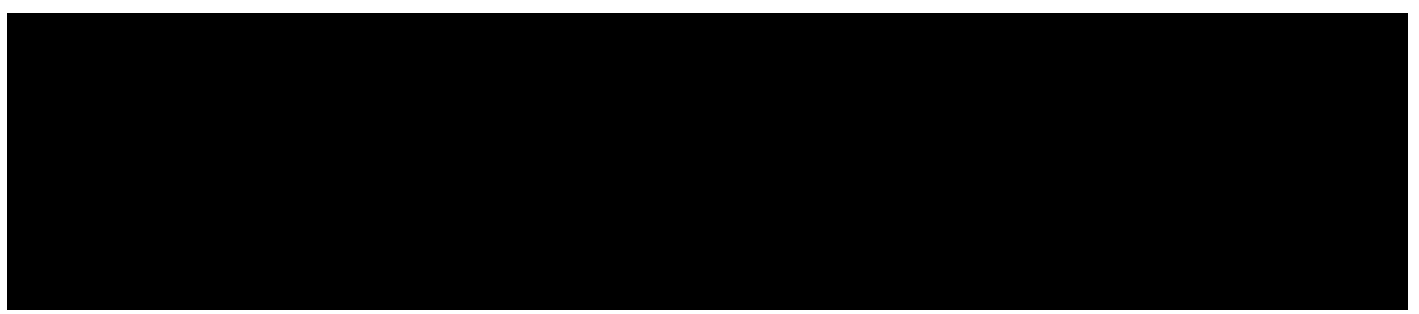

(d) 2005

(e) 2007

Figure 4: Signs for GOLD

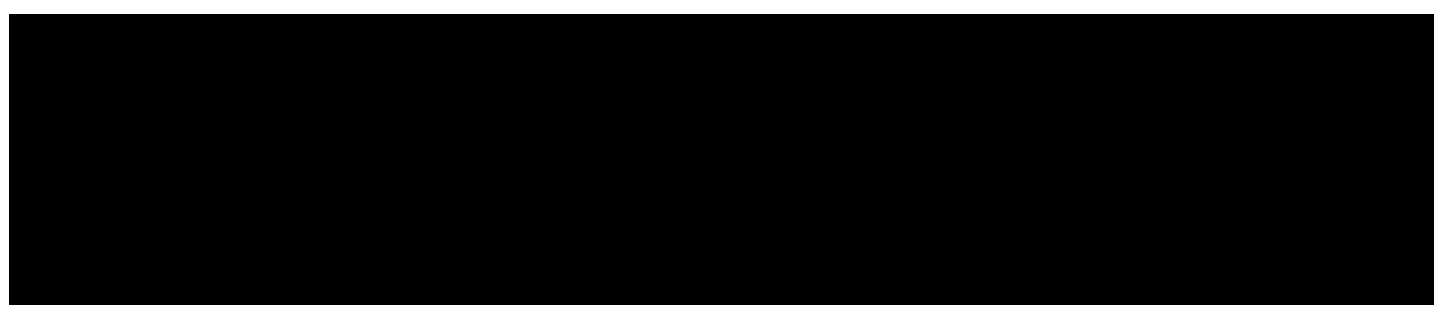
(a) 1972
(b) 1985
(c) 2004
(d) 2005
(e) 2007 
Figure 5: Signs for GREEN

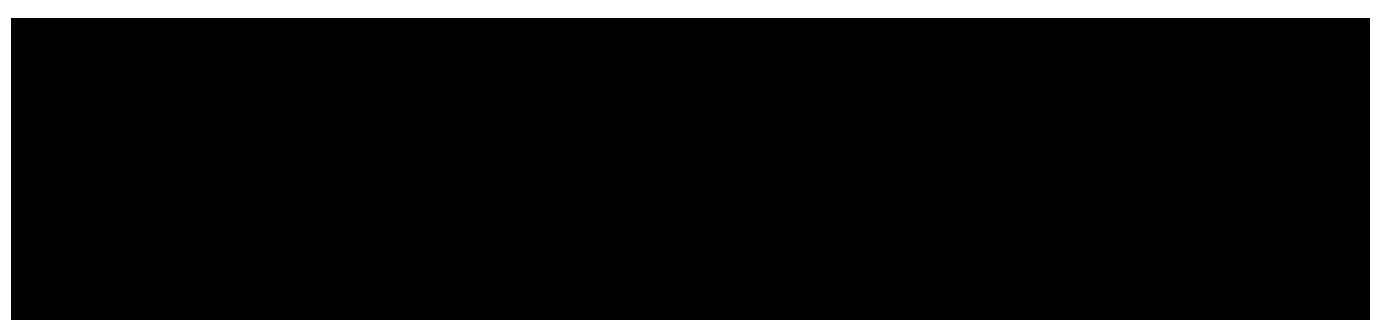

(a) 1972

(b) 1985

(c) 2004

(d) 2005

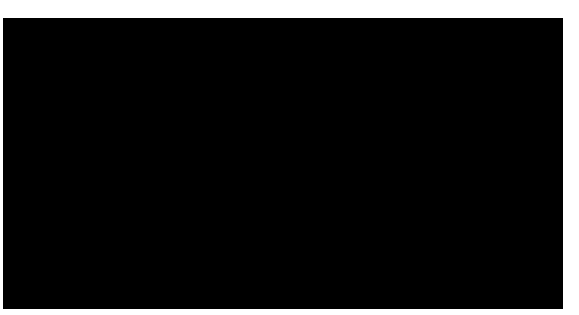

(e) 2007

Figure 6: Signs for GREY

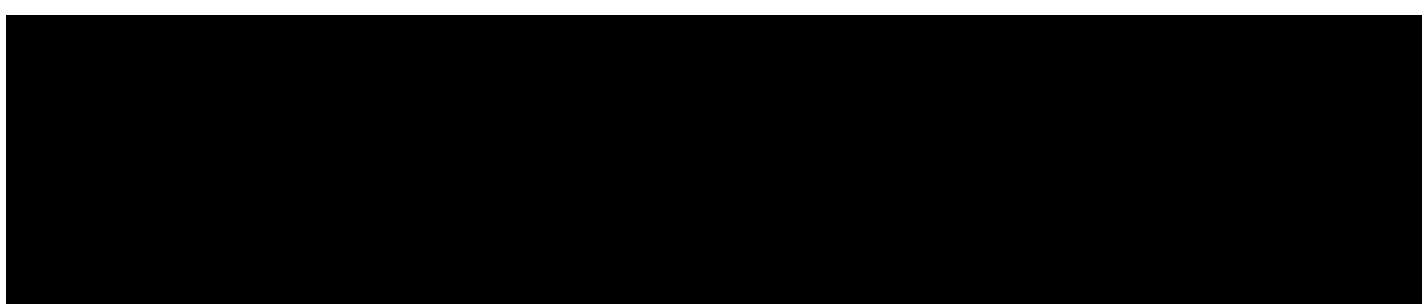

(a) 1972

(b) 1985

(c) 2004

(d) 2005

(e) 2007 
Figure 7: Signs for ORANGE

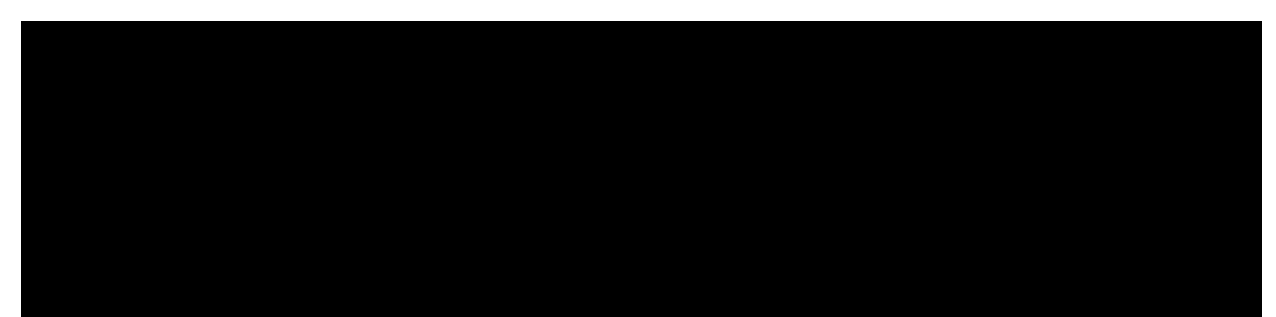

(a) 1972

(b) 1985

(c) 2004

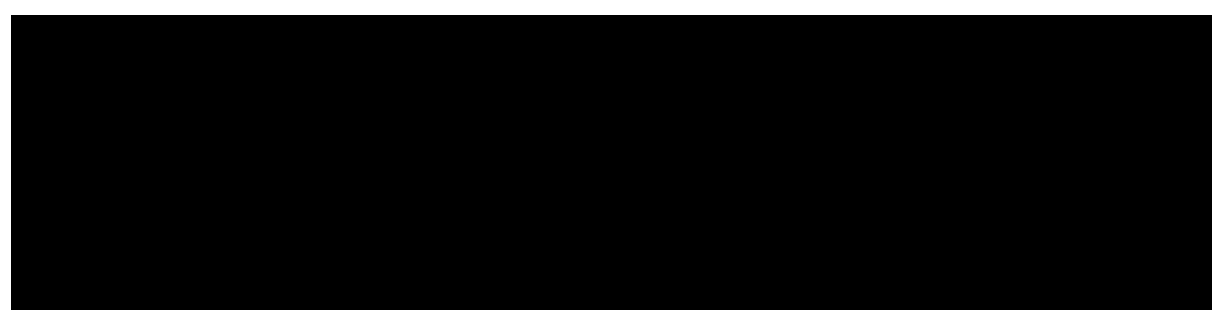

(d) 2005

(e) 2007

Figure 8: Signs for PINK

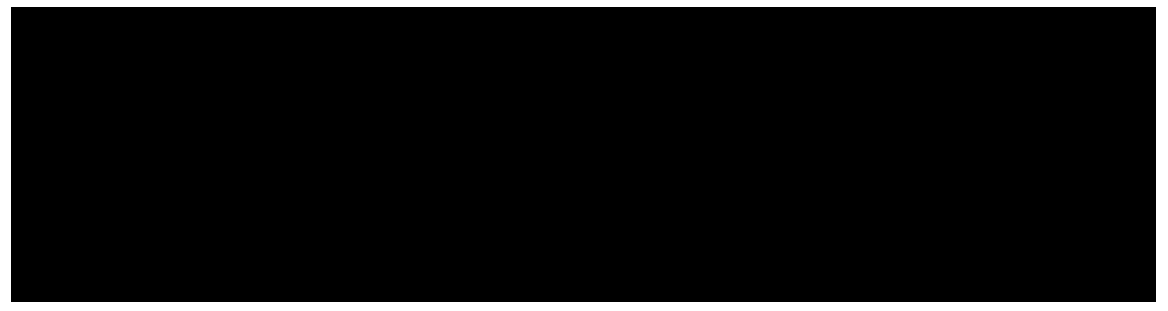
(a) 1972
(b) 1985
(c) 2004

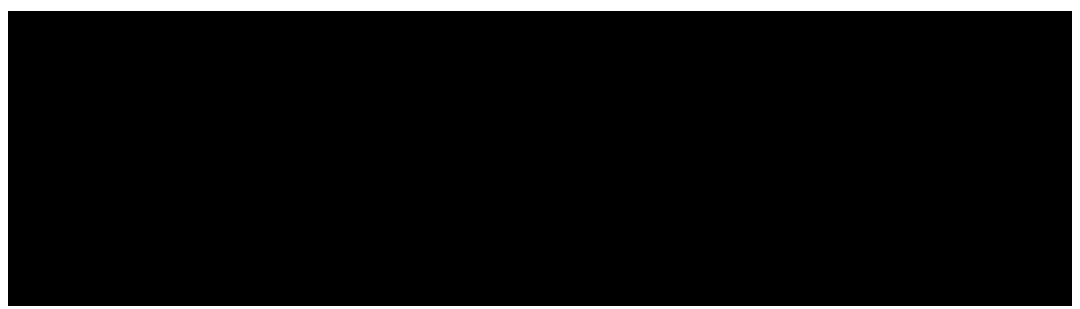

(d) 2005

(e) 2007 
Figure 9: Signs for PURPLE

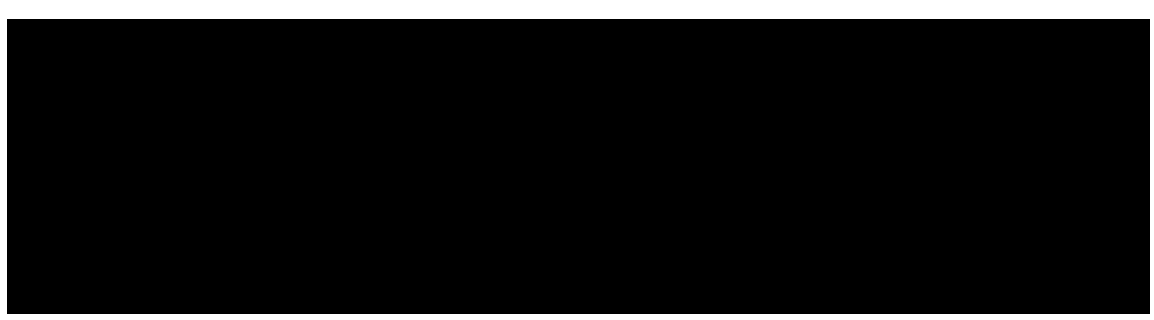
(a) 1972
(b) 1985
(c) 2004

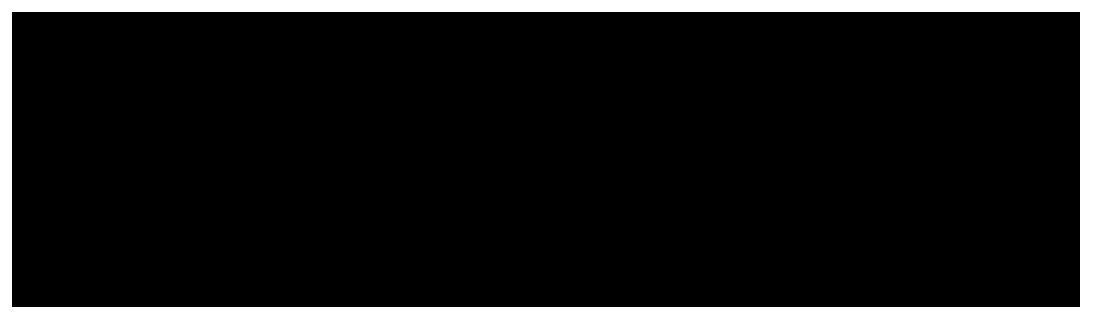

(d) 2005

(e) 2007

Figure 10: Signs for RED

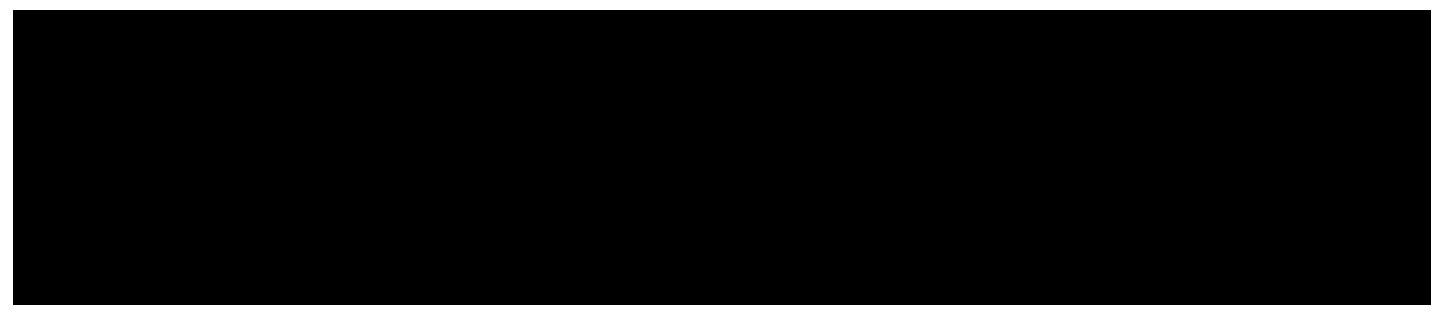
(a) 1972
(b) 1985
(c) 2004
(d) 2005
(e) 2007

Figure 11: Signs for SILVER
(a) 1972
(b) 1985
(c) 2004
(d) 2005
(e) 2007 
Figure 12: Signs for WHITE

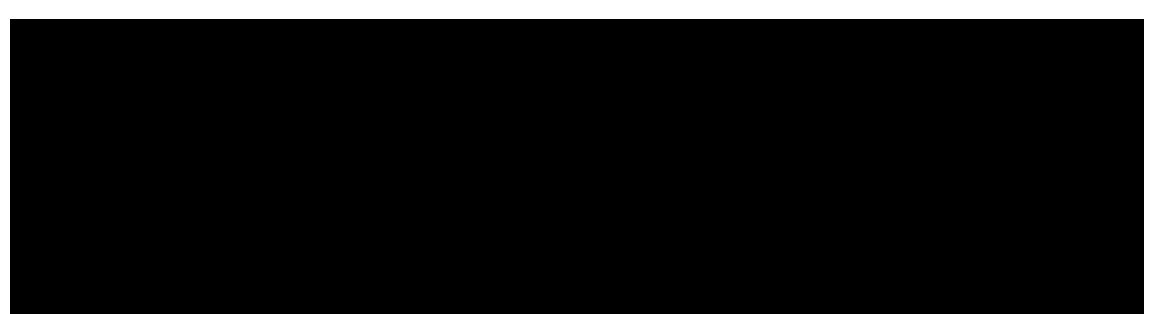

(a) 1972

(b) 1985

(c) 2004

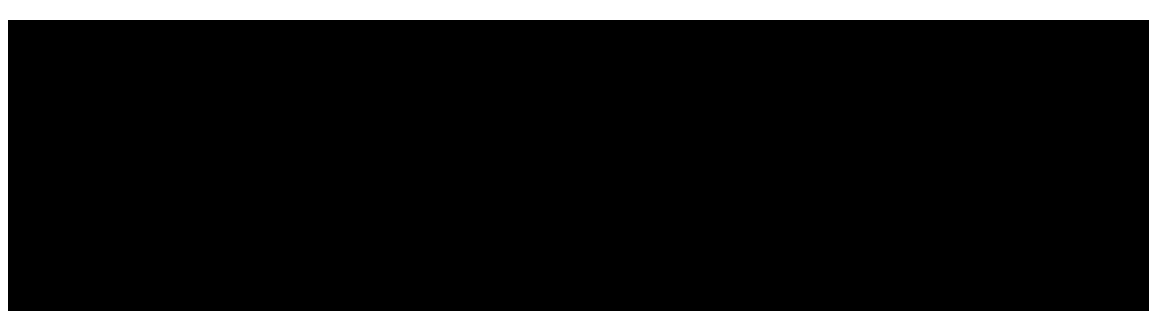

(d) 2005

(e) 2007 


\section{POSSIBLE HOMONYMS}

Figure 13: Signs for COFFEE (brown)

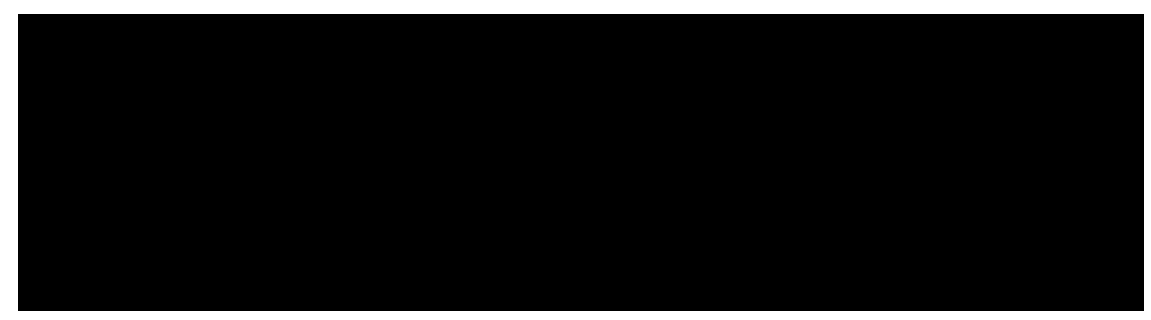
(a) 1972
(b) 1985
(c) 2004

(d) 2005

(e) 2007

Figure 14: Signs for GRAPE (purple)

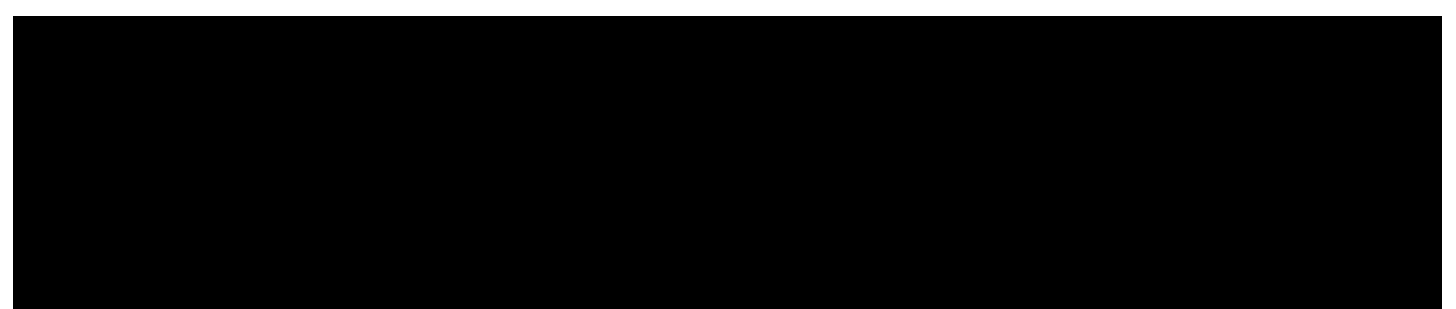
(a) 1972
(b) 1985
(c) 2004
(d) 2005
(e) 2007

Figure 15: Signs for HUNG-HOM, a local place name (red)
(a) 1972
(b) 1985
(c) 2004
(d) 2005
(e) 2007 
Figure 16: Signs for ORANGE (fruit)

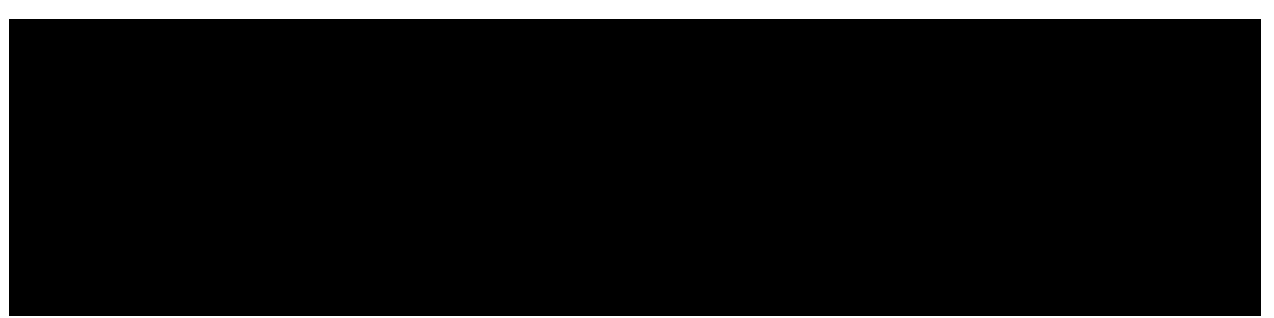

(a) 1972

(b) 1985

(c) 2004

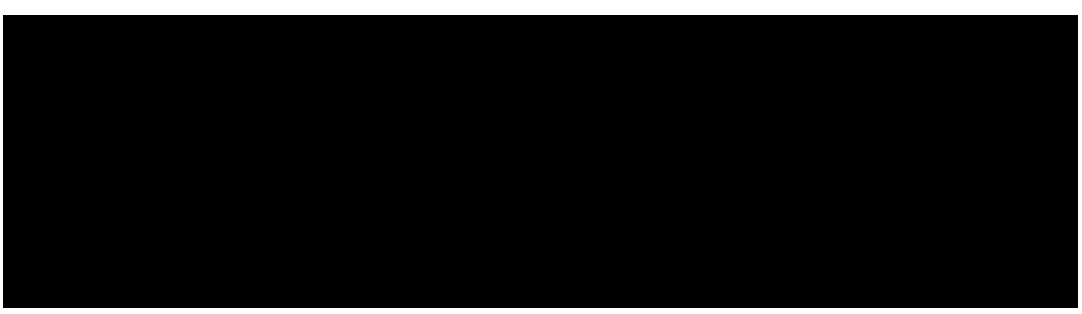

(d) 2005

(e) 2007 


\section{KINSHIP TERMS}

Figure 17: Signs for FATHER

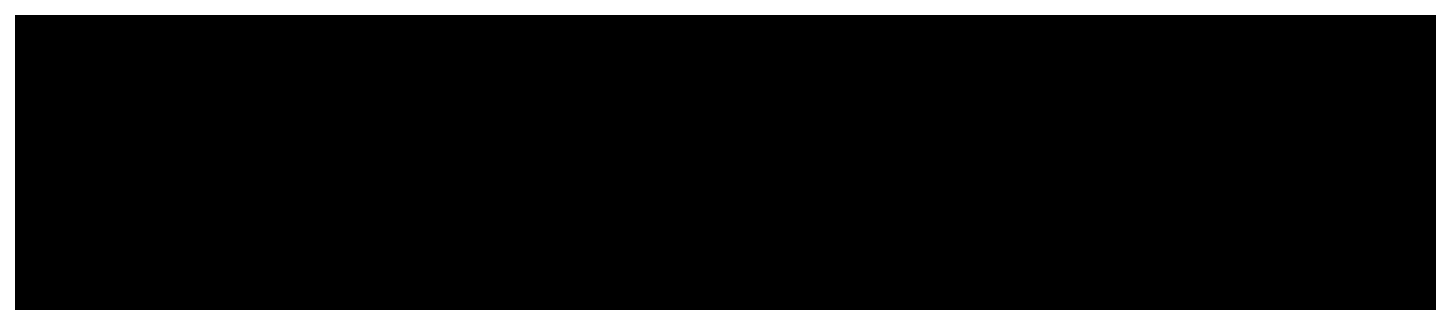
(a) 1972
(b) 1985
(c) 2004
(d) 2005
(e) 2007

Figure 18: Signs for MOTHER

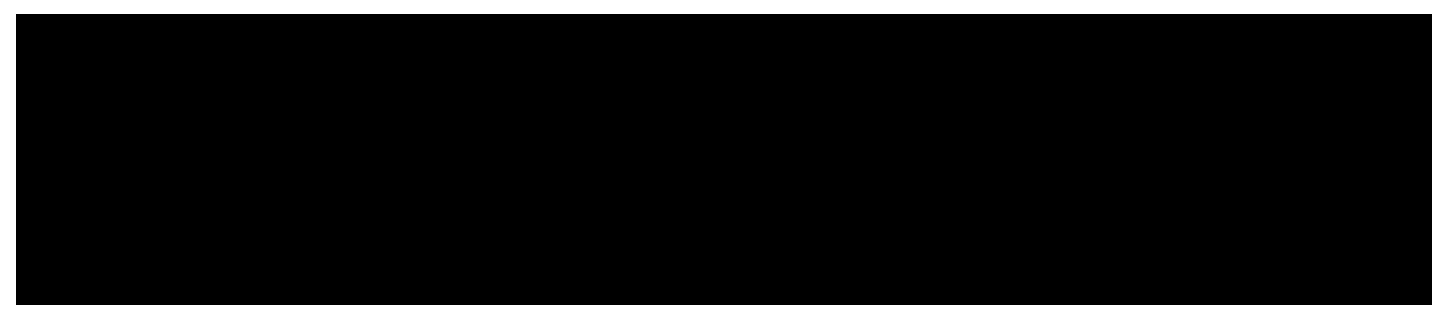
(a) 1972
(b) 1985
(c) 2004
(d) 2005
(e) 2007

Figure 19: Signs for ELDER-BROTHER

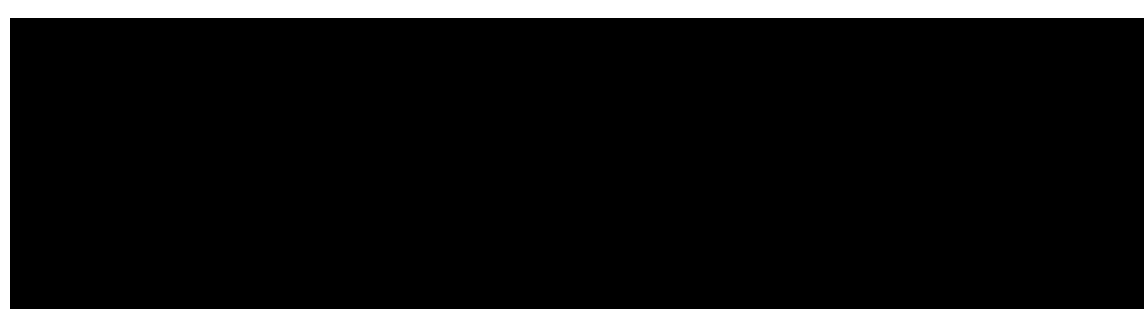
(a) 1972
(b) 1985
(c) 2004

(d) 2005

(e) 2007 
Figure 20: Signs for ELDER-SISTER

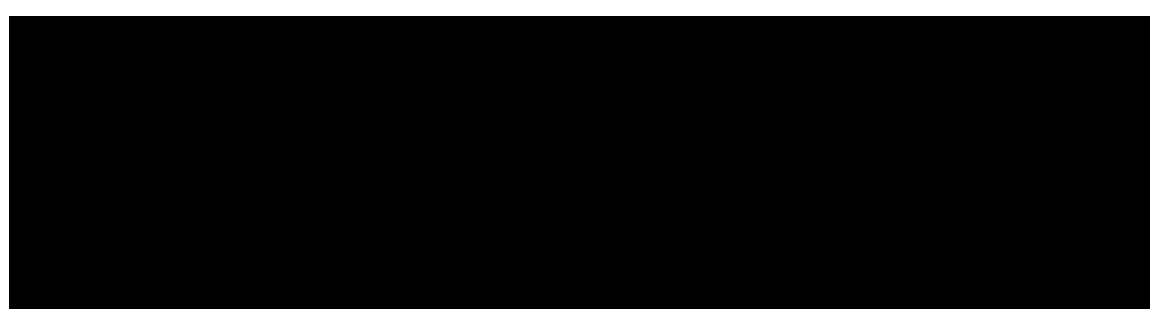
(a) 1972
(b) 1985
(c) 2004

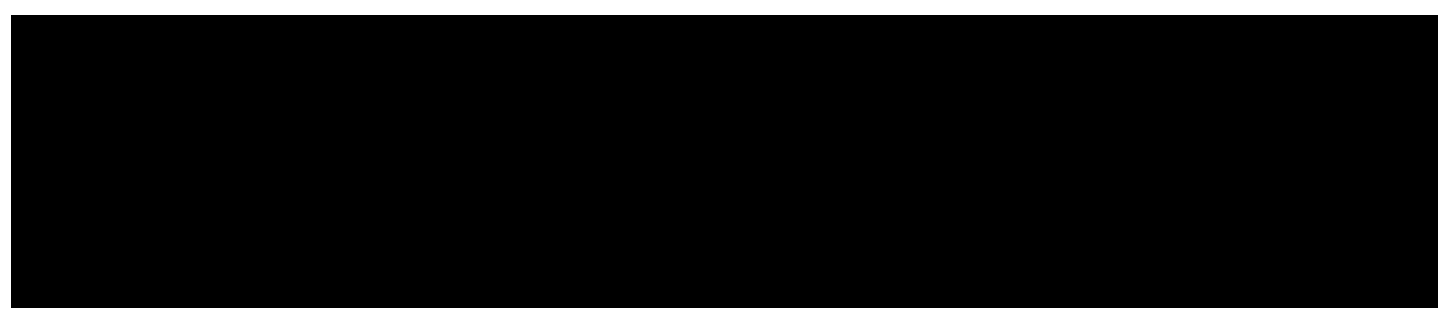

(d) 2005

(e) 2007

Figure 21: Signs for YOUNGER-BROTHER

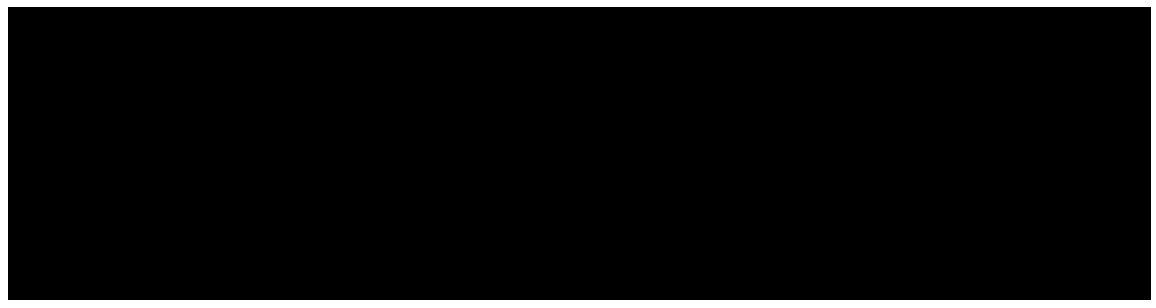
(a) 1972
(b) 1985
(c) 2004

(d) 2005

(e) 2007 
Figure 22: Signs for YOUNGER-SISTER

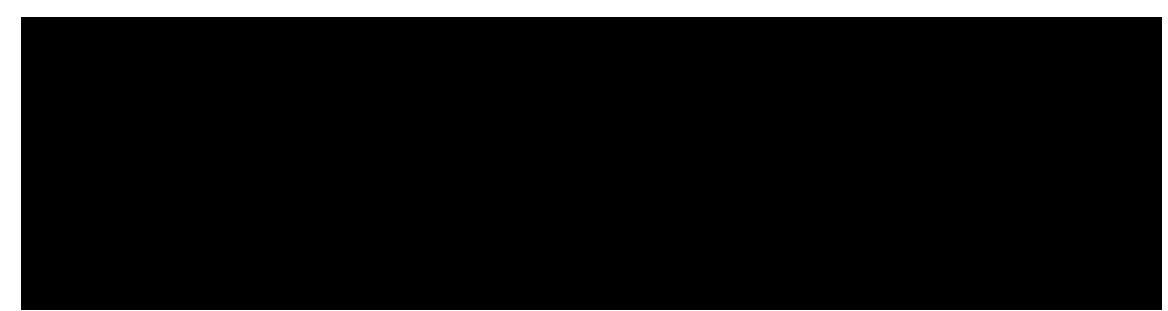
(a) 1972
(b) 1985
(c) 2004

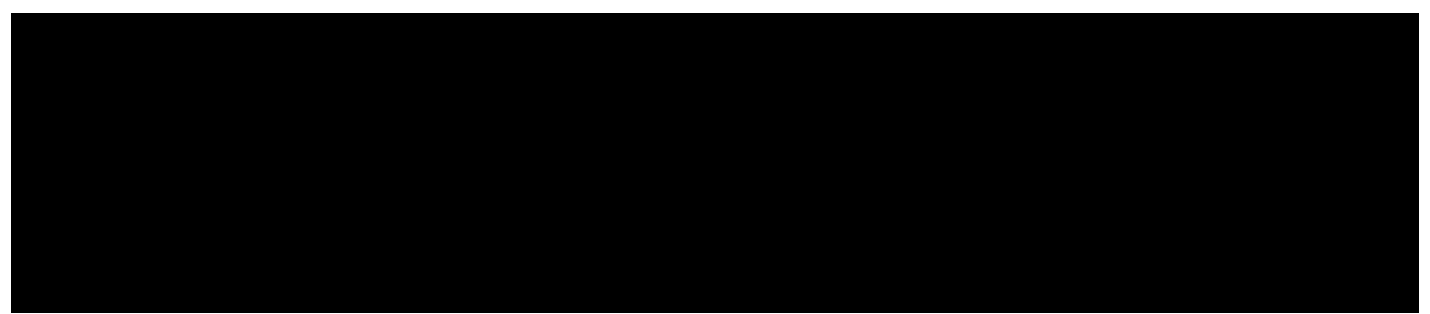

(d) 2005

(e) 2007

Figure 23: Signs for RELATIVES

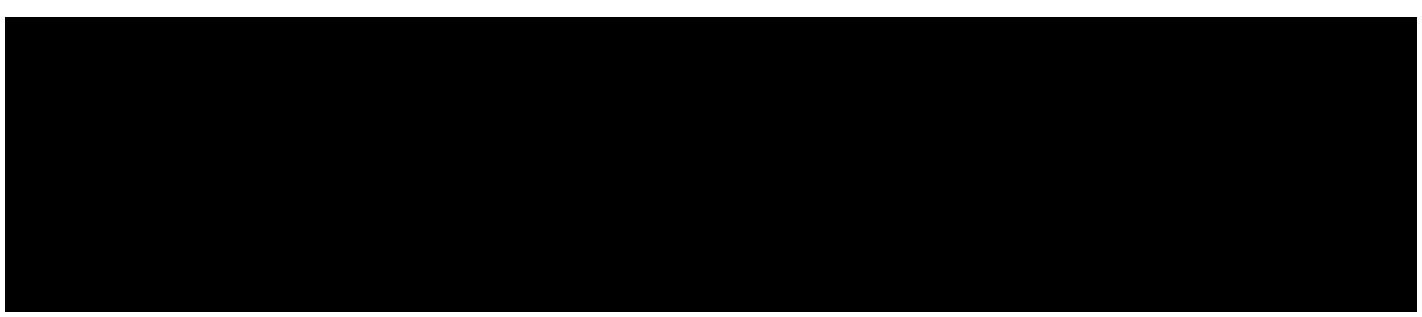
(a) 1972
(b) 1985
(c) 2004
(d) 2005
(e) 2007 


\section{NUMBERS}

Figure 24: Signs for THREE

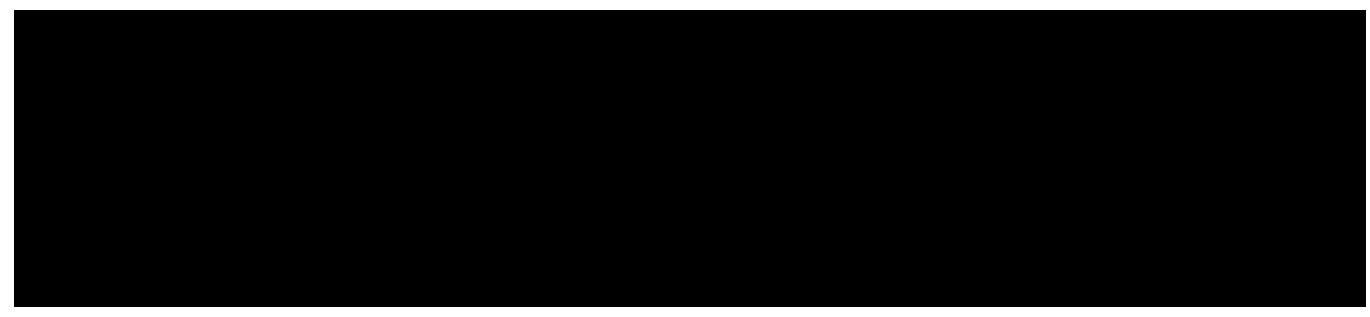

(a) 1972

(b) 1985

(c) 2004

(d) 2005

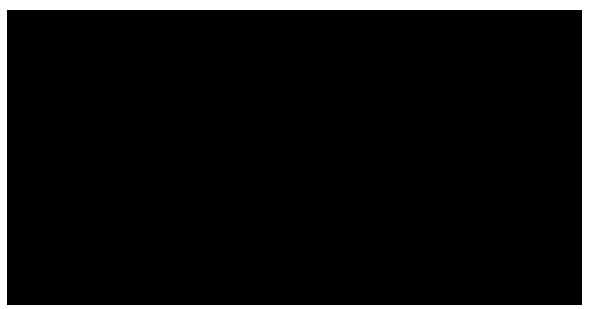

(e) 2007

Figure 25: Signs for SEVEN

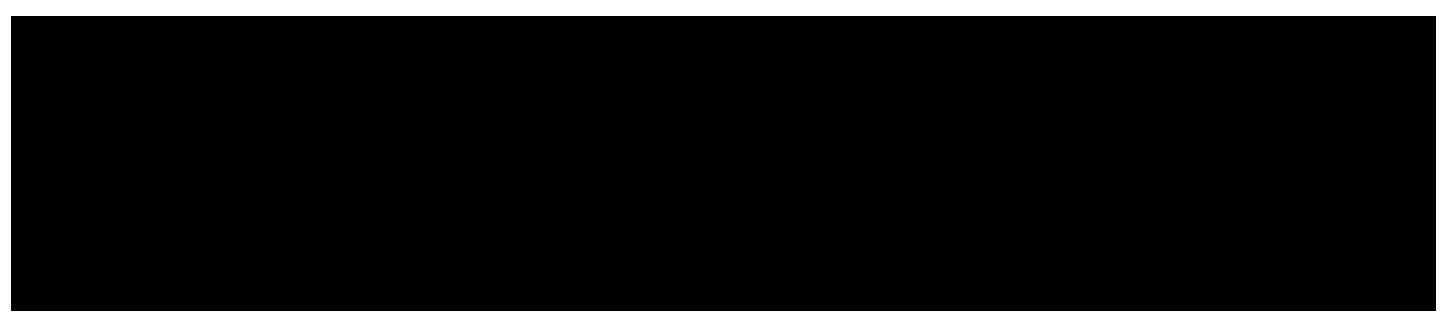
(a) 1972
(b) 1985
(c) 2004
(d) 2005
(e) 2007

Figure 26: Signs for EIGHT

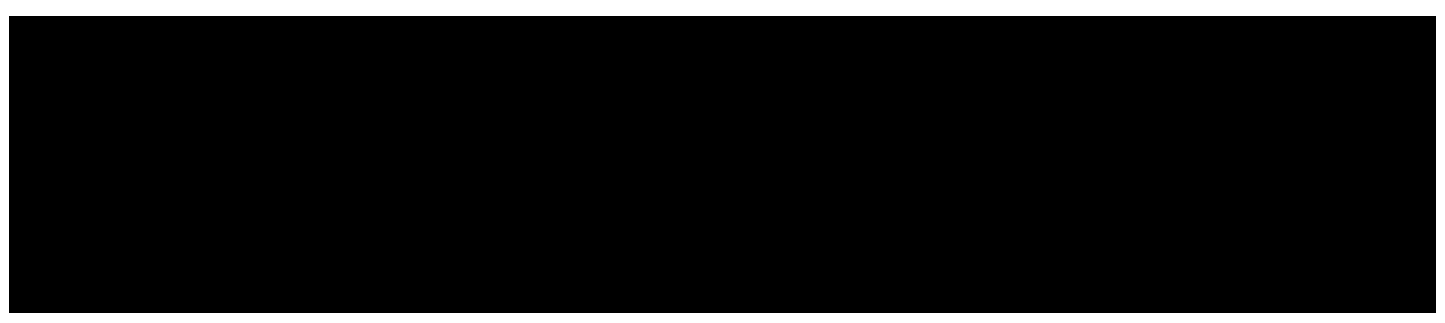
(a) 1972
(b) 1985
(c) 2004
(d) 2005
(e) 2007 
Figure 27: Signs for ELEVEN

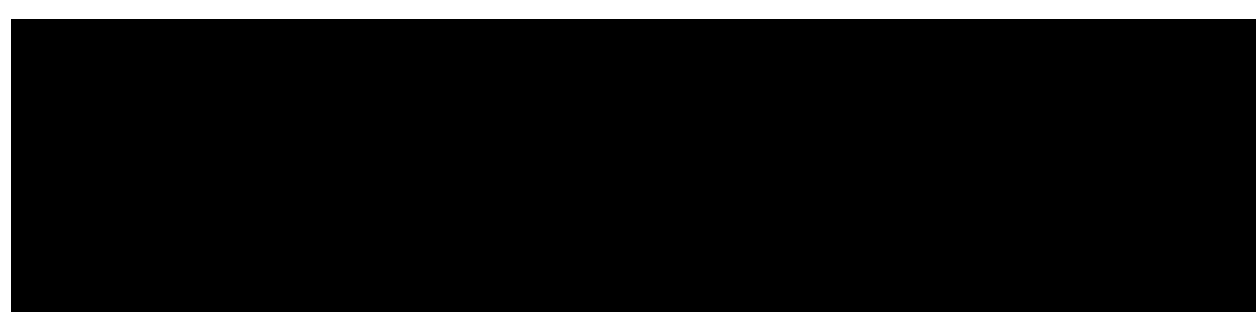
(a) 1972
(b) 1985
(c) 2004

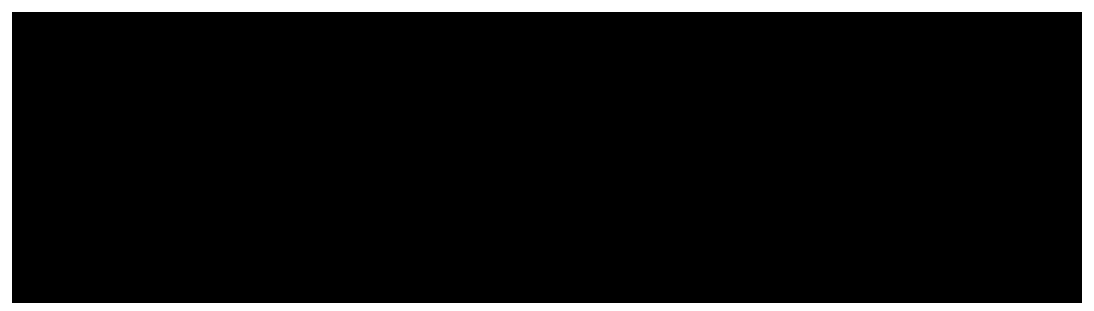

(d) 2005

(e) 2007

Figure 28: Signs for THIRTEEN

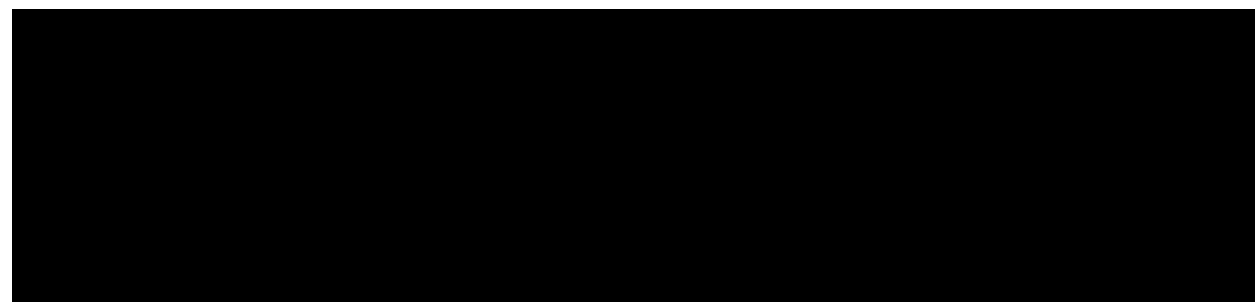

(a) 1972

(b) 1985

(c) 2004

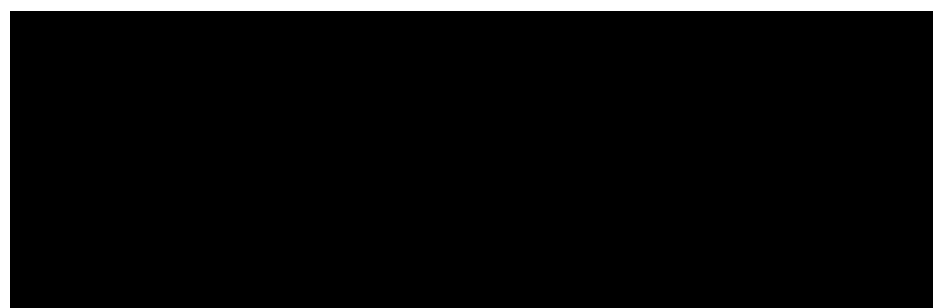

(d) 2005

(e) 2007 
Figure 29: Signs for FIFTEEN

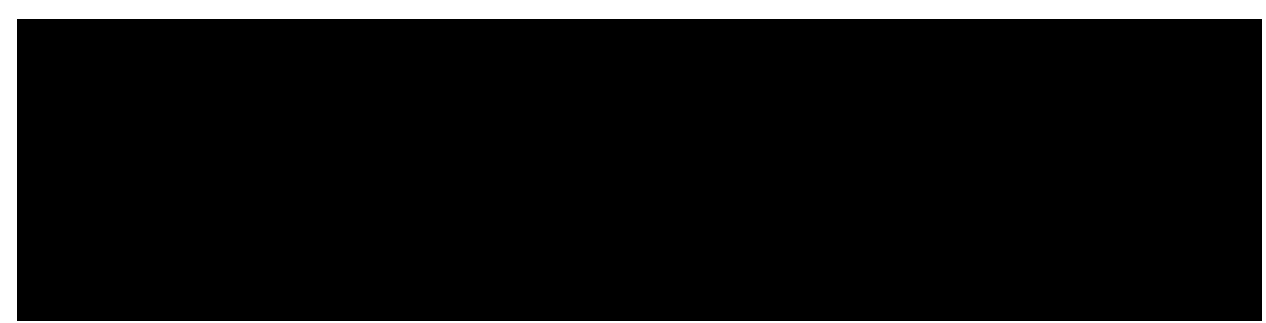

(a) 1972

(b) 1985

(c) 2004

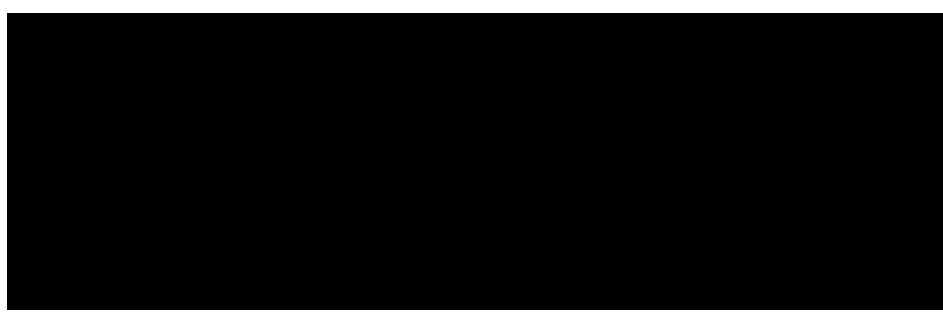

(d) 2005

(e) 2007

Figure 30: Signs for SEVEntEen

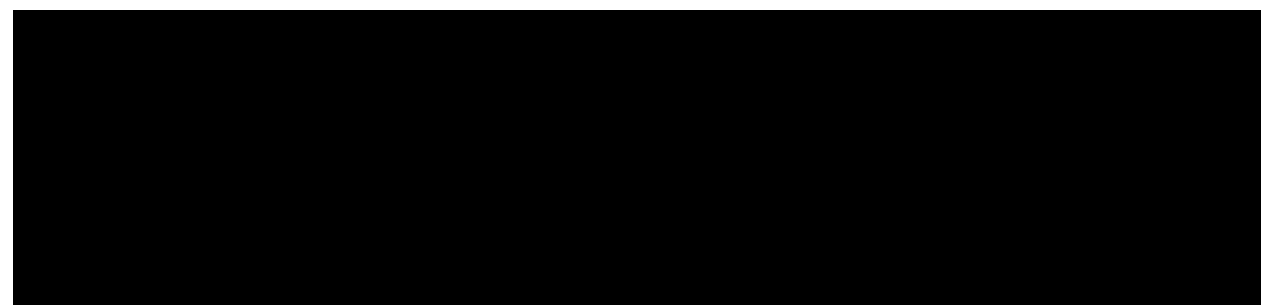

(a) 1972

(b) 1985

(c) 2004

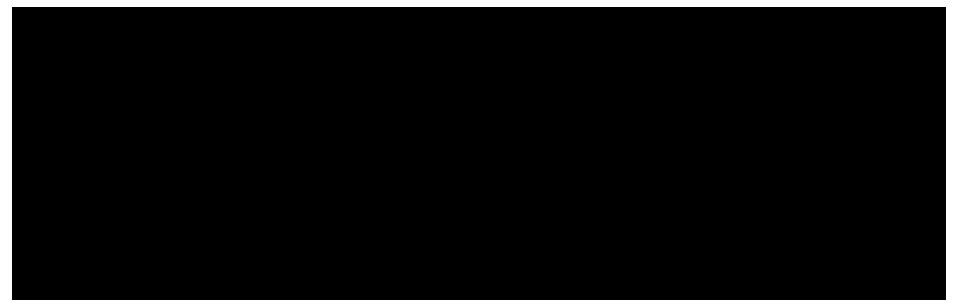

(d) 2005

(e) 2007 
Figure 31: Signs for EIGHTEEN

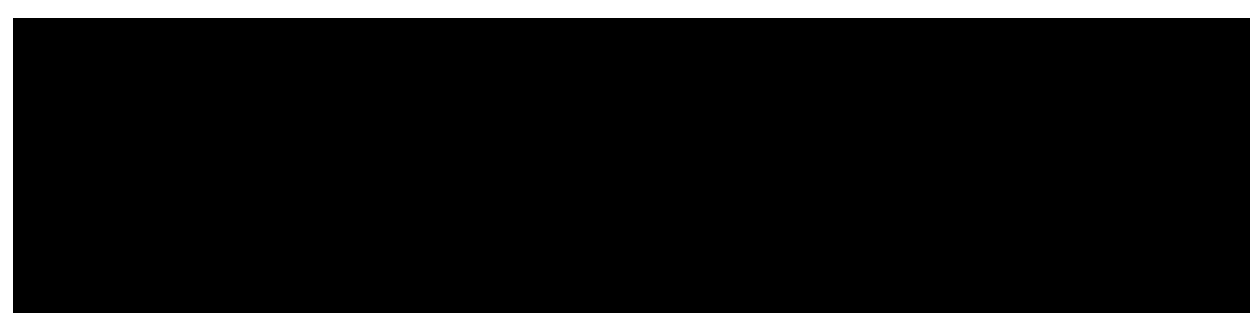
(a) 1972
(b) 1985
(c) 2004

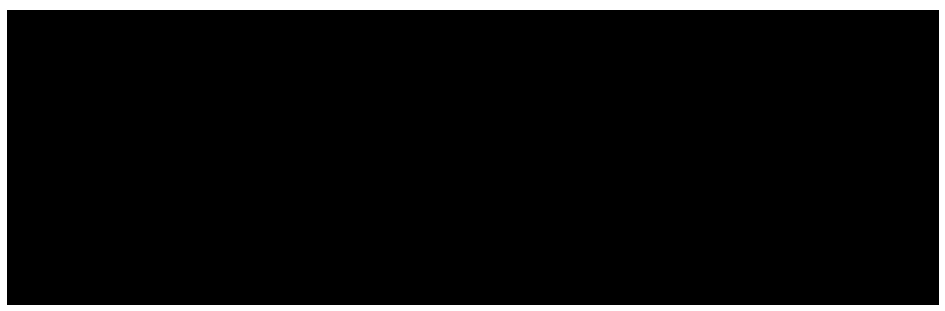

(d) 2005

(e) 2007

Figure 32: Signs for TWENTY

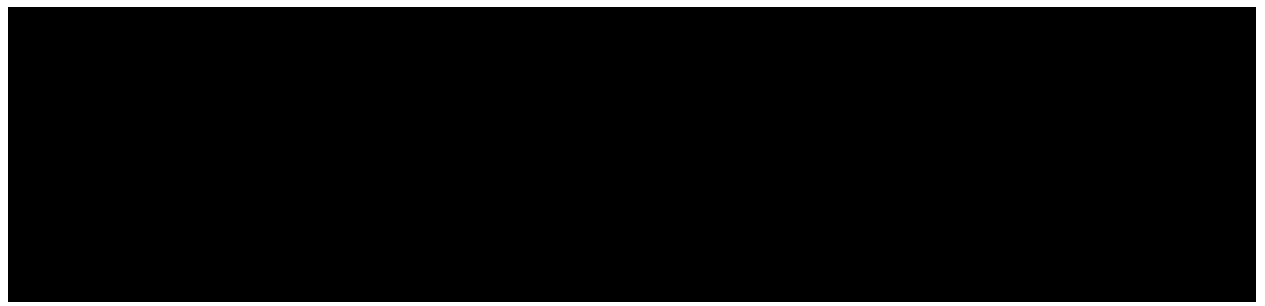

(a) 1972

(b) 1985

(c) 2004

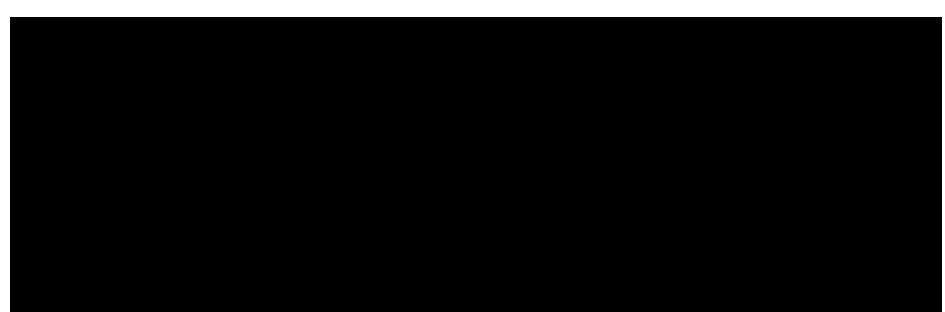

(d) 2005

(e) 2007 
Figure 33: Signs for TWENTY-TwO

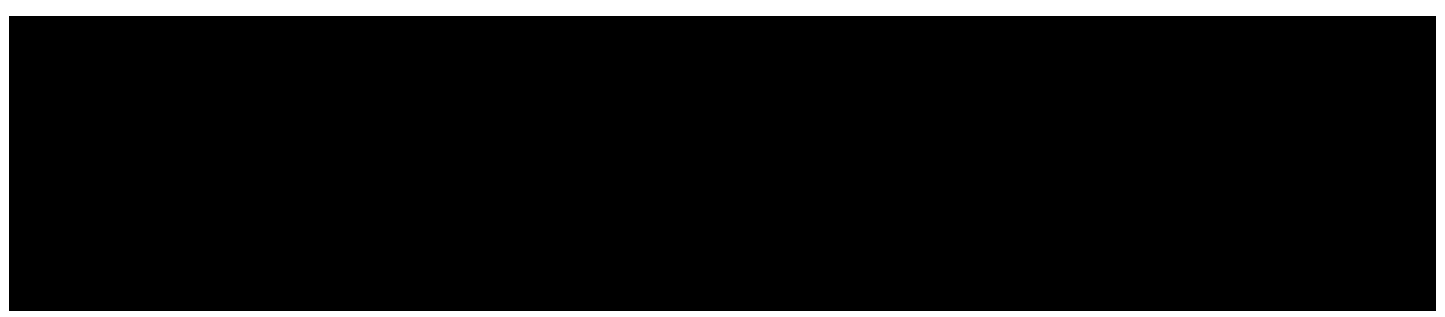
(a) 1972
(b) 1985
(c) 2004
(d) 2005
(e) 2007

Figure 34: Signs for TWENTY-THREE

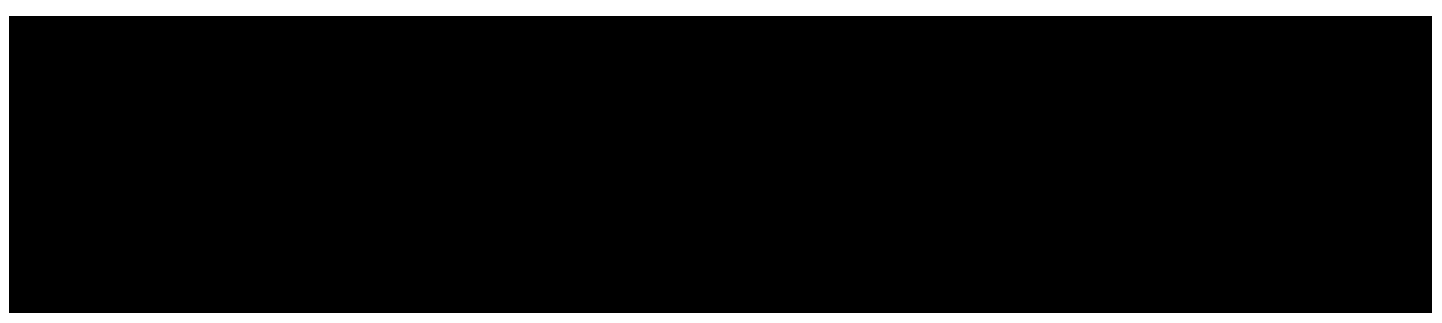
(a) 1972
(b) 1985
(c) 2004
(d) 2005
(e) 2007

Figure 35: Signs for TWENTY-EIGHT

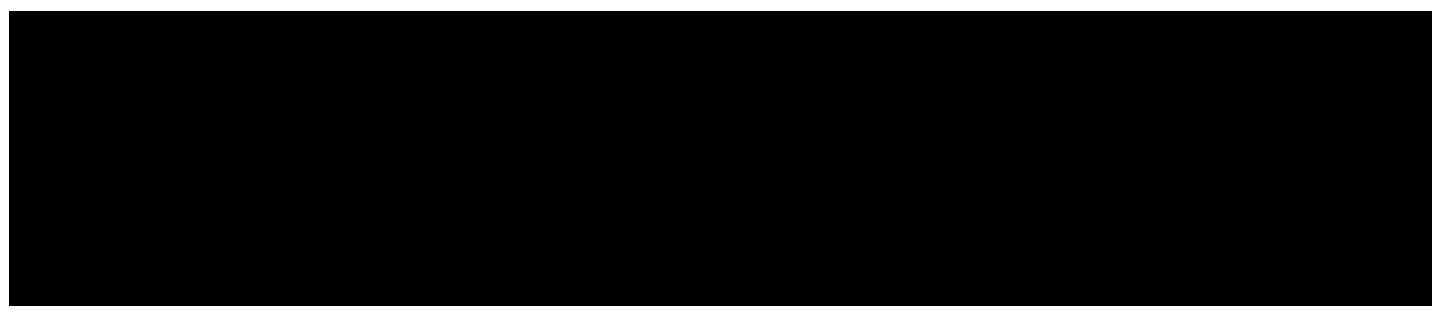
(a) 1972
(b) 1985
(c) 2004
(d) 2005
(e) 2007 
Figure 36: Signs for THIRTY

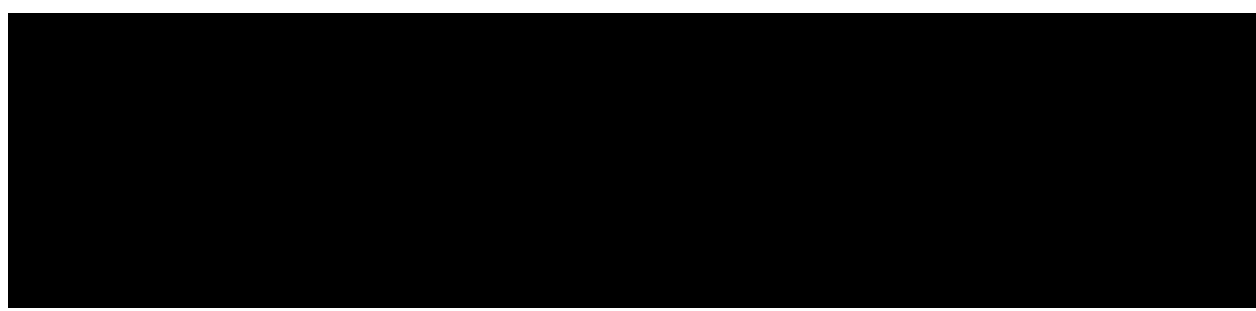
(a) 1972
(b) 1985
(c) 2004

(d) 2005

(e) 2007

Figure 37: Signs for HUNDRED

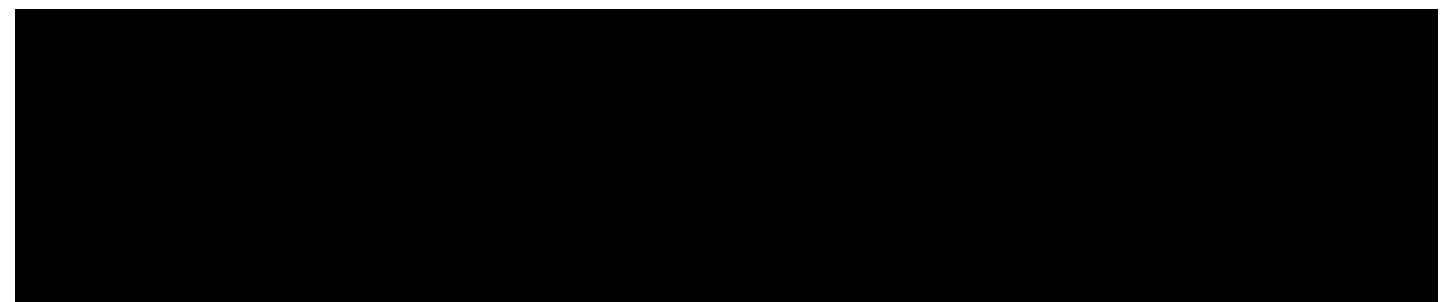
(a) 1972
(b) 1985
(c) 2004
(d) 2005
(e) 2007 


\section{COUNTRIES/REGIONS}

Figure 38: Signs for AUSTRALIA

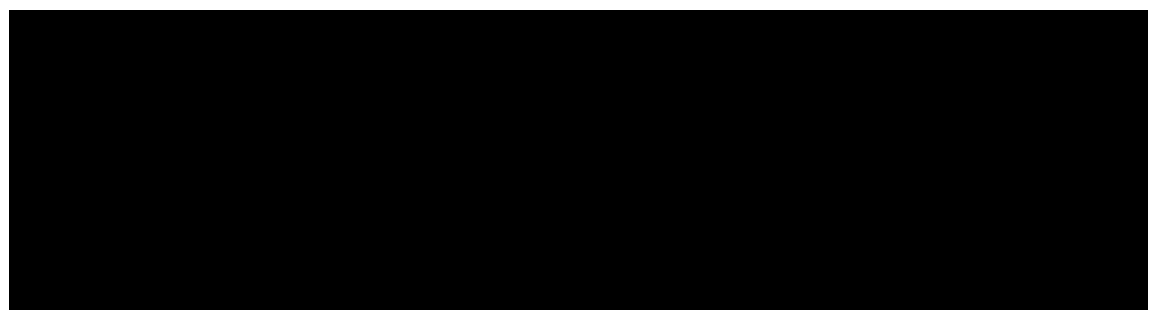
(a) 1972
(b) 1985
(c) 2004

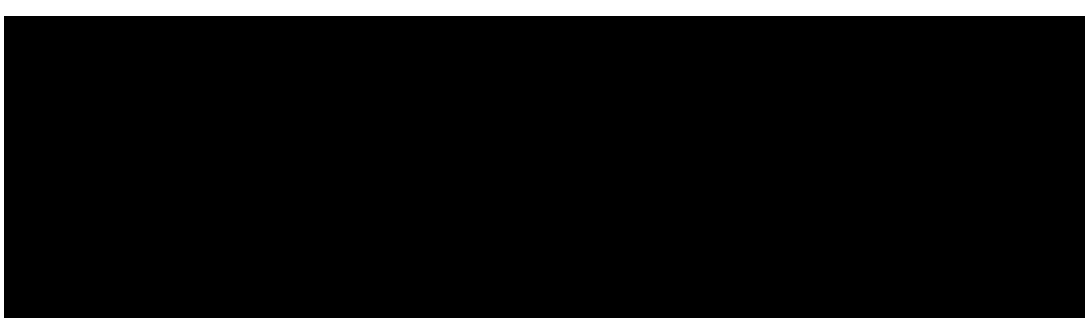

(d) 2006

(e) 2007

Figure 39: Signs for BEIJING

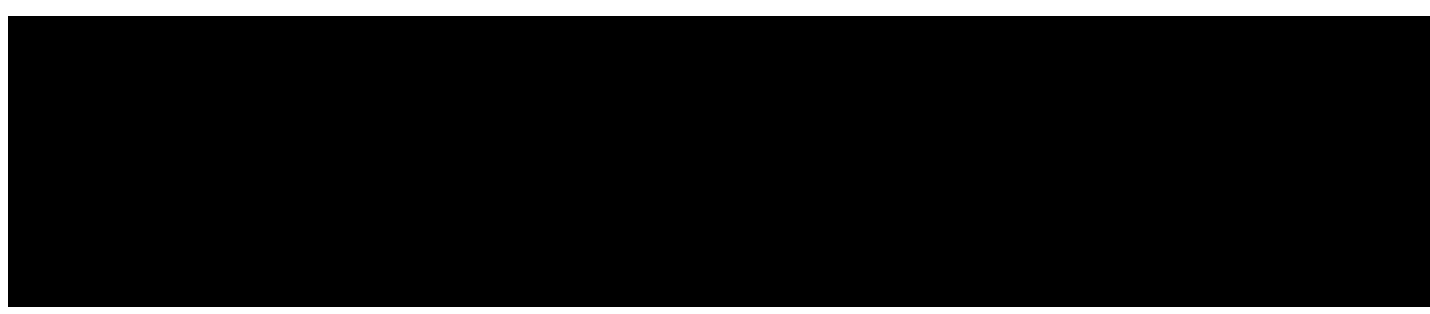
(a) 1972
(b) 1985
(c) 2004
(d) 2005
(e) 2007

Figure 40: Signs for GUANGZHOU

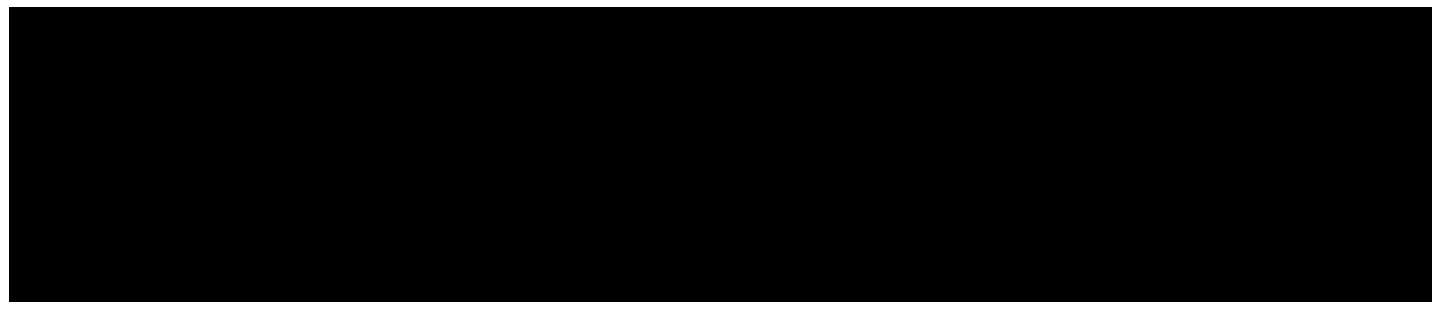
(a) 1972
(b) 1985
(c) 2004
(d) 2005
(e) 2007 
Figure 41: Signs for INDIA

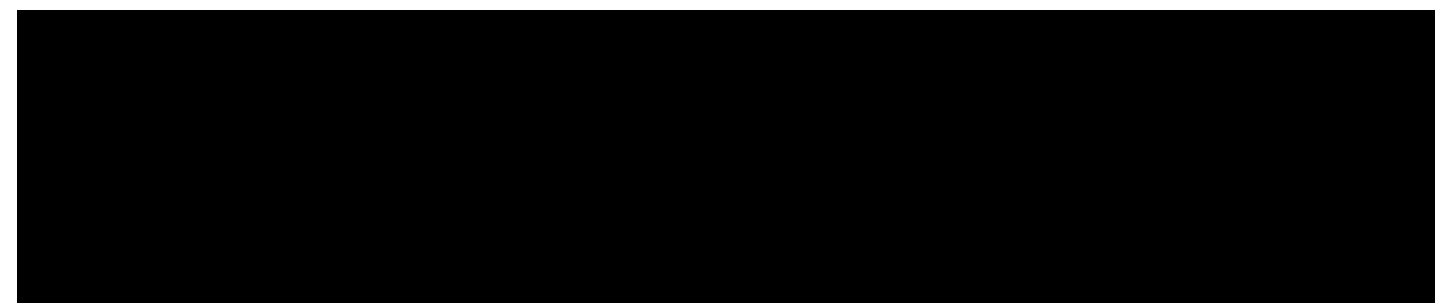
(a) 1972
(b) 1985
(c) 2004
(d) 2006
(e) 2007

Figure 42: Signs for ITALY

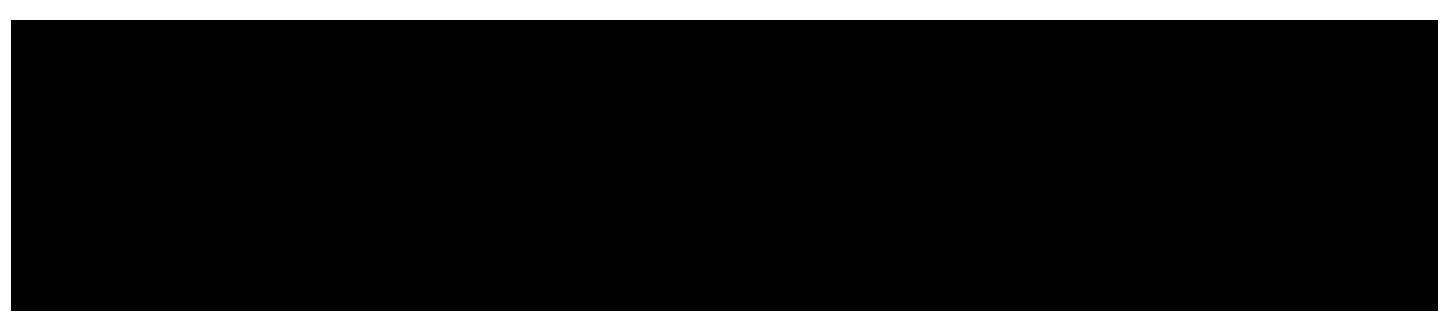
(a) 1972
(b) 1985
(c) 2004
(d) 2006
(e) 2007

Figure 43: Signs for KOREA

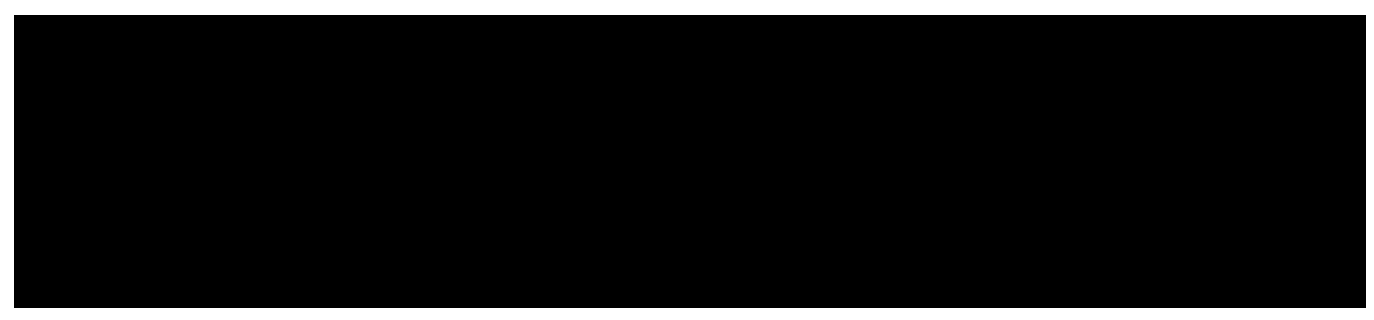

(a) 1972

(b) 1985

(c) 2004

(d) 2005

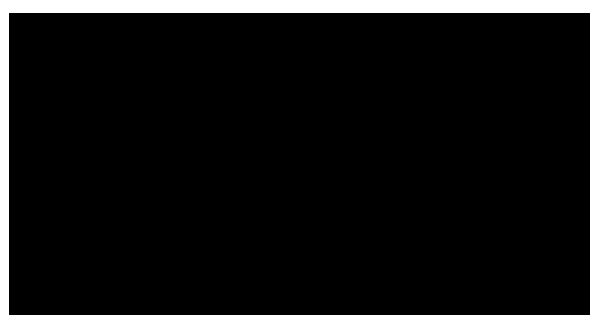

(e) 2007 
Figure 44: Signs for TAIWAN

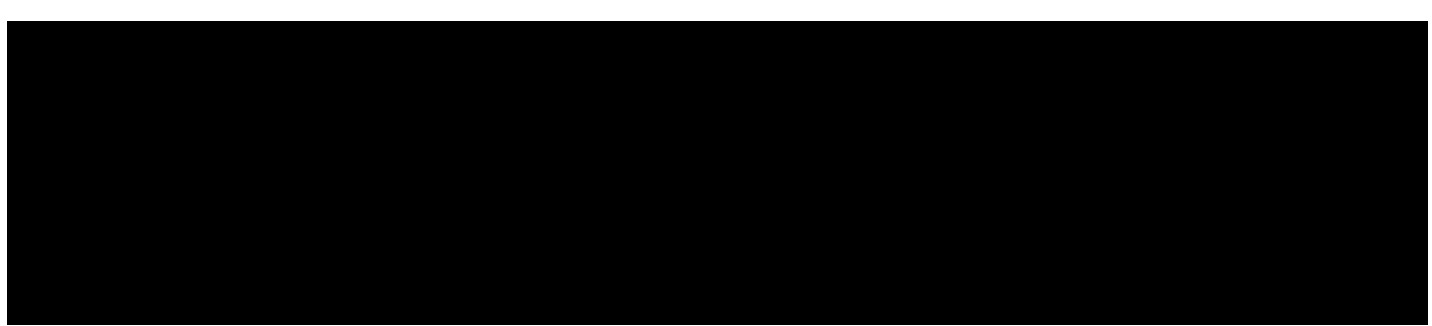

(a) 1972

(b) 1985

(c) 2004

(d) 2005

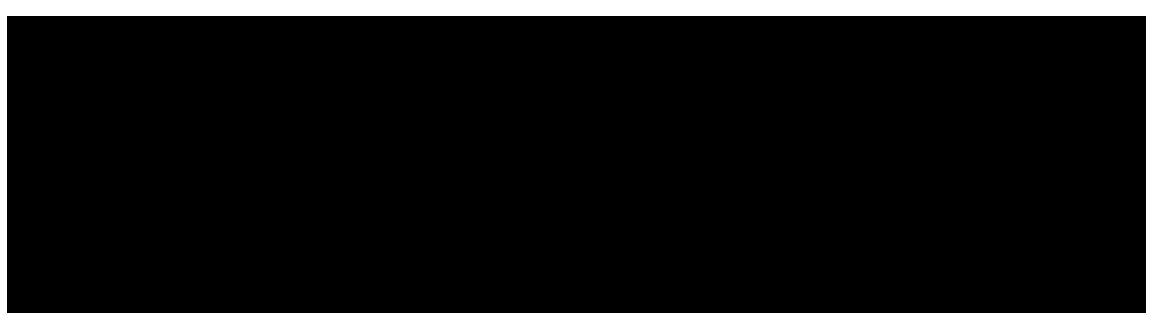

(e) 2007

Figure 45: Signs for VIETNAM

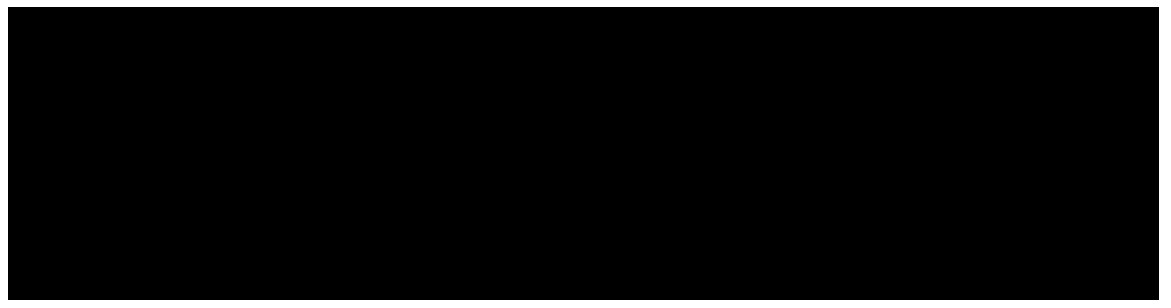

(a) 1972

(b) 1985

(c) 2004

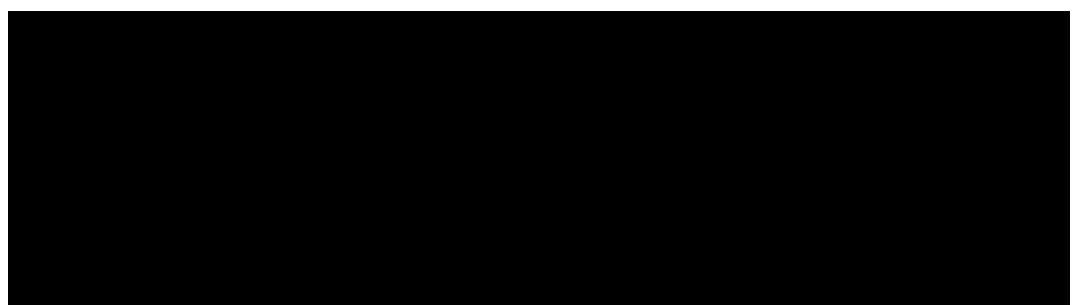

(d) 2006

(e) 2007 


\section{DEAF/HEARING}

Figure 46: Signs for DEAF

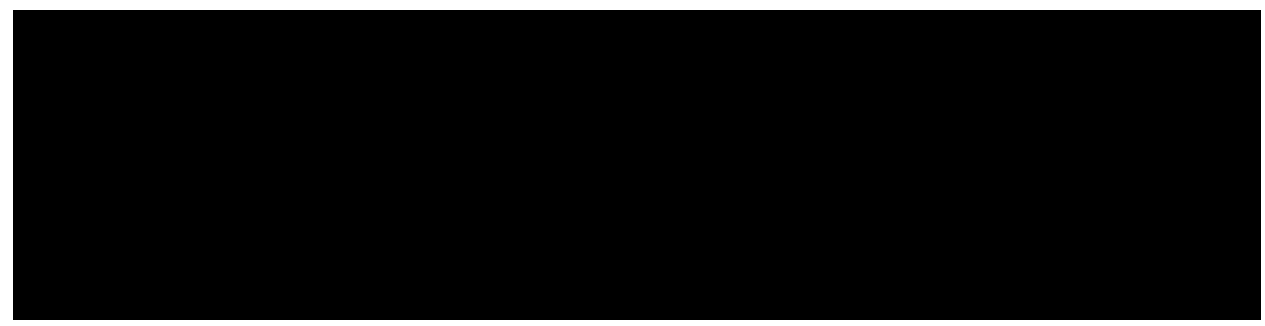
(a) 1972
(b) 1985
(c) 2004

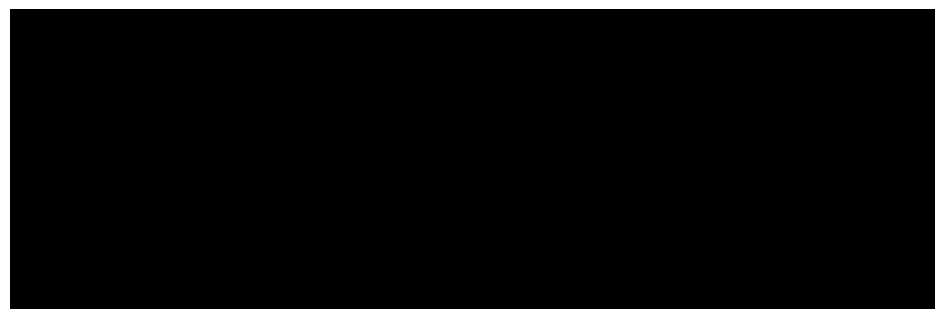

(d) 2005

(e) 2007

Figure 47: Signs for HEARING

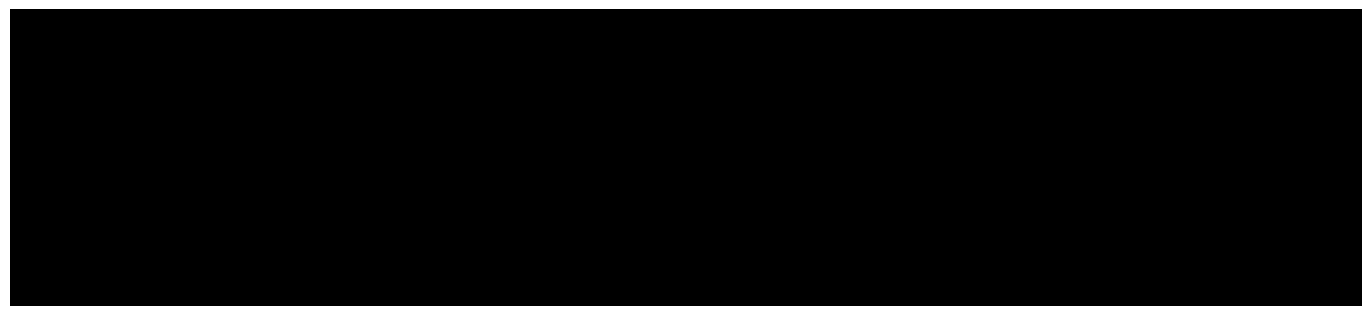

(a) 1972

(b) 1985

(c) 2004

(d) 2005

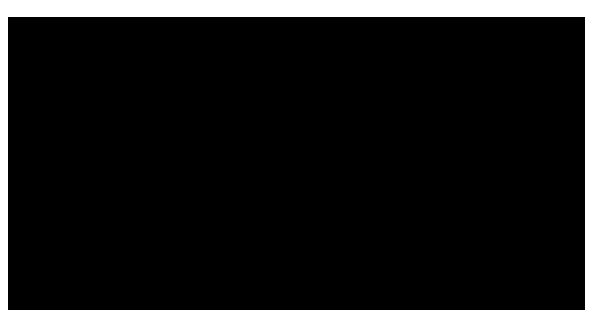

(e) 2007 